\title{
Erratum zu: 52. Tagung der Österreichischen Ophthalmologischen Gesellschaft, 2. bis 4. Juni 2011, Mayrhofen im Zillertal
}

Erratum zu: Spektrum Augenheilkd (2011) 2: 79-202

DOI 10.1007/s00717-011-0479-1

Sehr geehrte Leserinnen und Leser des Spektrum der Augenheilkunde,

durch einen bedauerlichen Irrtum wurde nicht die letztgültig korrigierte Fassung dieser Ausgabe abgedruckt.

Wir erlauben uns, Ihnen nachfolgend die nunmehr berichtigte Fassung übermitteln zu dürfen und entschuldigen uns bei Ihnen seitens des Verlags und der Schriftleitung für die dadurch entstandenen Umstände:

Tagung der Österreichischen Ophthalmologischen Gesellschaft

2. bis 4. Juni 2011, Mayrhofen im Zillertal 


\section{spektrum der augenheilkunde}

Zeitschrift der Österreichischen Ophthalmologischen Gesellschaft Journal of the Austrian Ophthalmic Society

Band 25 Heft 22011

Spektrum Augenheilkd (2011) 2: 79-202

DOI 10.1007/s00717-011-0478-2

๑ Springer-Verlag 2011

\section{TAGUNG DER ÖSTERREICHISCHEN OPHTHALMOLOGISCHEN GESELLSCHAFT}

2. bis 4. Juni 2011

MAYRHOFEN IM ZILLERTAL

A-ARVO - H-ARVO Science Day

Wissenschaftliche Sitzungen

Diagnostisches Konsil

State of the Art: Tumore des Auges

Wissenschaftliche Ausstellung

Vollversammlung der Österreichischen Ophthalmologischen Gesellschaft Industrieausstellung 


\section{Österreichische Ophthalmologische Gesellschaft}

In einer Festsitzung anlässlich des 50-jährigen Bestandes der Ophthalmologischen Gesellschaft in Wien am 22. Juni 1954 wurde die Gründung einer österreichischen augenärztlichen Gesellschaft beschlossen, damit eine international anerkannte Vertretung bestünde. Vom 14.-15. Mai 1955 fand dann in Wien die erste Tagung der Österreichischen Ophthalmologischen Gesellschaft - ÖOG statt. Präsident war damals Prof. Dr. A. Pillat.

\section{Ehrenmitglieder}

Prof. Dr. Karl Lindner (Wien) † 12. 5. 1961

Prof. Dr. Josef Meller (Wien) † 23. 11. 1968

Prof. Dr. Ludwig v. Sallmann (Bethseda) † 24. 9. 1975

Prof. Dr. Arnold Pillat (Wien) † 25. 9.1975

Prof. Dr. Wilhelm Rohrschneider (München) † 17. 6. 1966

Prof. Dr. Adolf Franceschetti (Genf) † 8. 3. 1968

Prof. Dr. Josef Böck (Wien) † 15. 6. 1985

Prof. Dr. h. c. Dr. Friedrich Hollwich (München) † 4. 9. 1991

Doz. Dr. Joannis Fronimopoulos (Athen) † 13. 9. 1995

Prof. Dr. Gian Battista Bietti (Rom) † 2. 3. 1977

Prof. Dr. Jules Francois (Gent) $†$ 13. 8. 1984

Prof. Dr. Wolfgang Jaeger (Heidelberg) † 15. 10. 1995

Prof. Dr. B. A. Klien-Moncreiff (Tucson) † 1. 12. 1978

Prof. Dr. Clemens Kronfeld (Tucson) † 1. 1. 1980

Prof. Dr. Fritz Blodi (Iowa) † 30. 10. 1996

Prof. Dr. h. c. Dr. Hans Sautter (Hamburg) † 7. 9. 1984

Prof. Dr. Karl Hruby (Wien) † 26. 9. 1995

Prof. Dr. Helmut Fanta (Wien) † 8. 11. 2001

Prof. Dr. Wolfgang Funder (Wien) † 2. 10. 1998
Prof. Dr. Arno Nover (Mainz) † 1. 7. 1994

Prof. Dr. Rudolf Witmer (Zürich) † 25. 8. 1992

Prof. Dr. Hanns-Jürgen Merté (München) † 13. 4. 2003

Prof. Dr. Alfred Huber (Zürich) † Juli 2006

Prof. Dr. Joachim Küchle (Münster) † 24. 9. 2005

OMR Dr. Walter Friess (Graz) † 1. 10. 1993

Prof. Dr. Udo Nemetz (Wien) † 7. 12. 2004

Prof. Dr. Hans Slezak (Wien)

OMR Hofrat Prim. Prof. Dr. Franz Todter (St. Pölten)

Prof. Dr. Daniel Vörösmarthy (Budapest)

Prof. Dr. Peter Speiser (St. Gallen)

Dr. Adele Rabensteiner (Graz) † 12. 12. 1988

Dr. Franz Della Casa (Burgdorf) $† 2010$

Prof. Dr. Jörg Draeger (Hamburg)

MR. Prim. Prof. Dr. Paul Drobec (Wien)

Prof. Dr. Heinz Freyler (Wien)

Prim. Prof. Dr. Josef Stepanik (Wien) † 28. 2. 2010

Prim. Prof. Dr. Georg Zehetbauer (Wien) $\dagger$ 17. 12. 2009

Dr. Ladislaus Batthyany-Strattmann (Kittsee) † 22. 1. 1931

\section{Vorstand der ÖOG 2010/2011}

Präsident: Prim. Univ.-Prof. Dr. Günther Grabner

Ständige wiss. Sekretärin: Prim. Univ.-Prof. Dr. Susanne Binder

Schriftführer: Priv.-Doz. Dr. Matthias Bolz

Stv.Schriftführer: Univ.-Prof. Dr. Michael Georgopoulos

Kassier: MR Prim. Prof. Dr. Paul Drobec

Vorstand der Augenklinik Graz: Univ.-Prof. Dr. Andreas Wedrich

Vorstand der Augenklinik Innsbruck: Univ.-Prof. Dr. Nikolaos E. Bechrakis

Vorstand der Augenklinik Wien: Univ.-Prof. Dr. Ursula M. Schmidt-Erfurth

Schriftleiter des Spektrum der Augenheilkunde: MR Prim. Prof. Dr. Paul Drobec

Vorsitzender des Fachgruppenbeirates: Dr. Peter Gorka

Vorsitzende des Consilium Strabologicum Austriacum: VR, Univ.-Prof. Dr. Andrea Langmann

Vorsitzender der Vereinigung kontaktlinsenanpassender Augenärzte: MR Dr. Herbert Schuster

Bundesfachgruppenobfrau: OMR Dr. Helga Azem

Primarärztevertreter: Prim. Univ.-Prof. Dr. Th. Michael Radda

Referent für Öffentlichkeitsarbeit: Dr. Carl Glittenberg

Mittelbauvertreter: Dr. Gerald Schmidinger und Dr. Stefan Felke

\section{Fachgruppenbeirat der ÖOG 2010/2011}

OMR Dr. Helga Azem (Wien)

Dr. Karl Hubel (Kärnten)

Dr. Peter Gorka - Vorsitzender (Niederösterreich)

Dr. Karl Riedler (Oberösterreich)

Dr. Willi Jordan (Salzburg)
OMR Dr. Roderich Fellner (Steiermark)

Dr. Walter Mair (Tirol)

Dr. Andrea Guggenberger (Vorarlberg)

Dr. Günther Tölly (Burgenland) 
$\equiv$ 
言 


\section{Inhaltsverzeichnis}

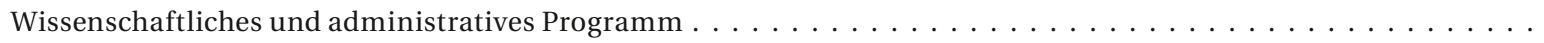

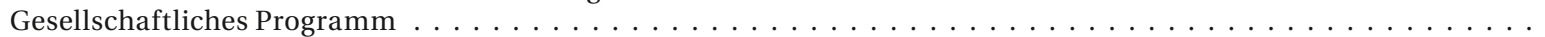

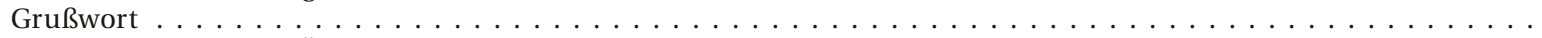

Vollversammlung der Österreichischen Ophthalmologischen Gesellschaft ～. . . . . . . . . . . . . . . . .

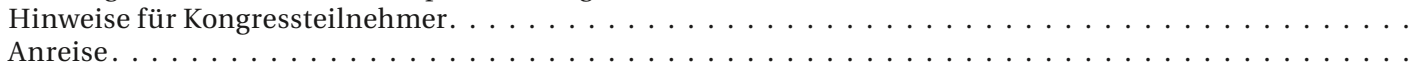

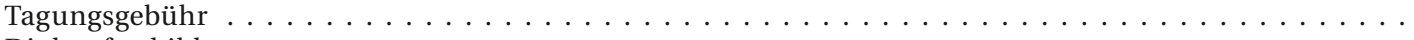

Diplomfortbildungsprogramm. . . . . . . . . . . . . . . . . . . . .

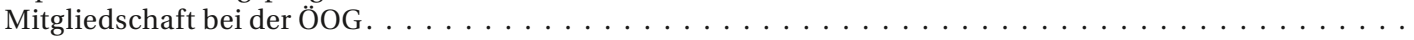

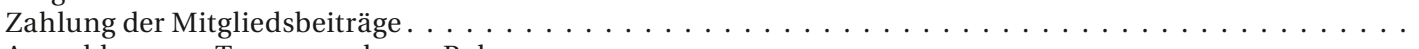

Anmeldung zur Tagung und zum Rahmenprogramm $\ldots \ldots \ldots \ldots \ldots \ldots \ldots \ldots \ldots$

Quartierbestellungen.

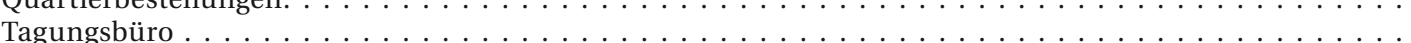

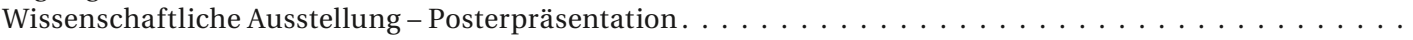

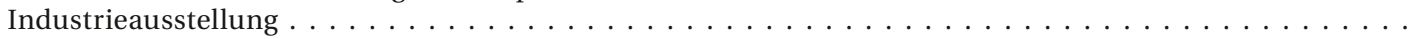

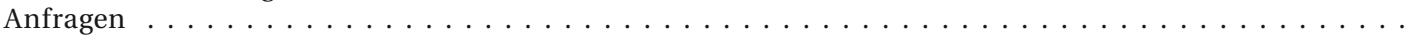

Hinweise für die Vortragenden. . . . . . . . . . . . . . . . . . . .

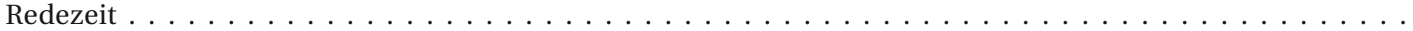

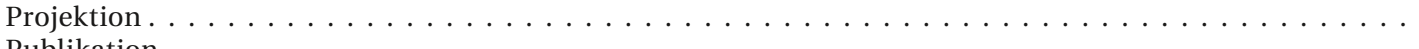

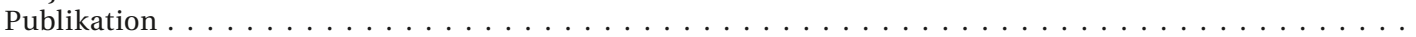

Diagnostisches Konsil . . . . . . . . . . . . . . . . . . . . . . . . . .

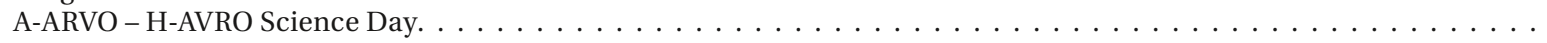

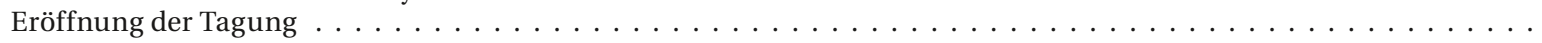

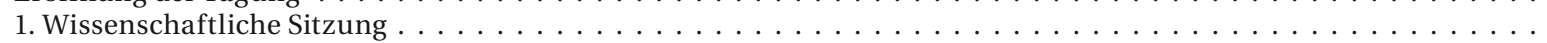

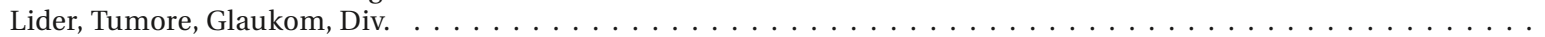

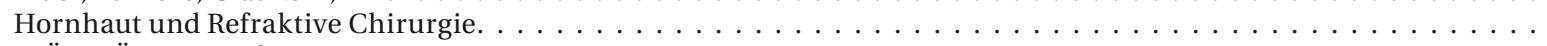

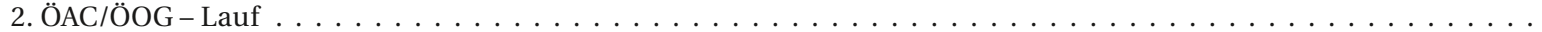

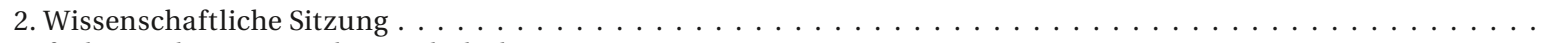

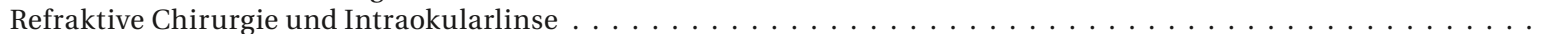

Vollversammlung der Österreichischen Ophthalmologischen Gesellschaft . . . . . . . . . . . . . . .

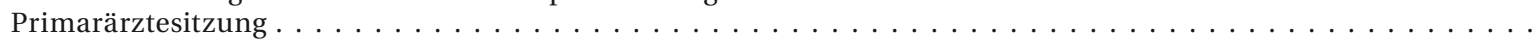

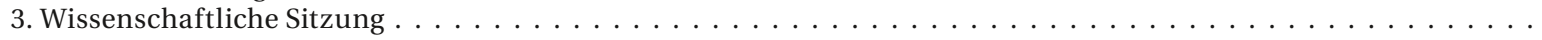

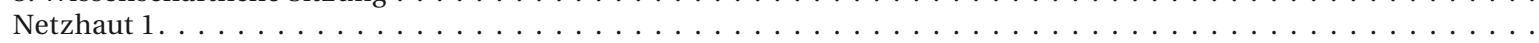

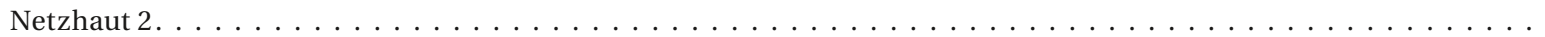

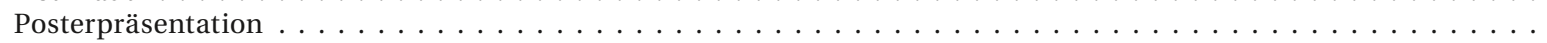

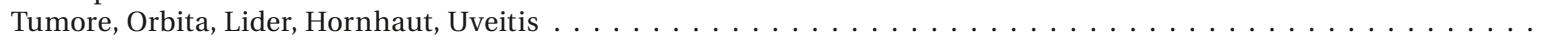

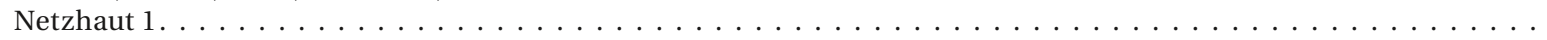

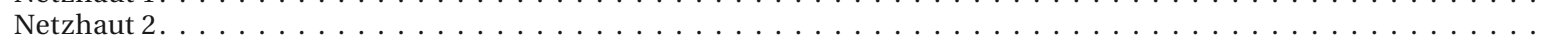

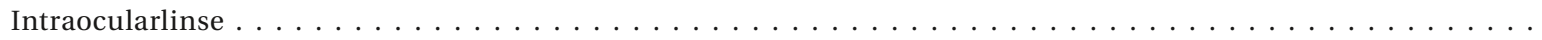

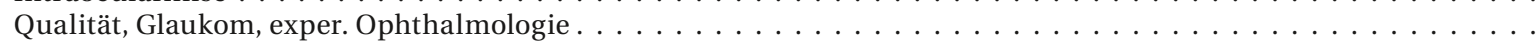

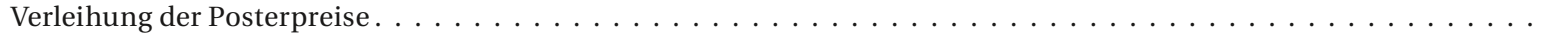

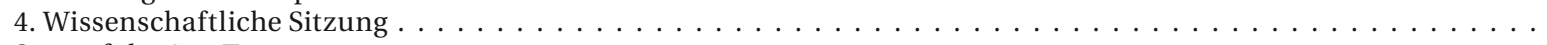

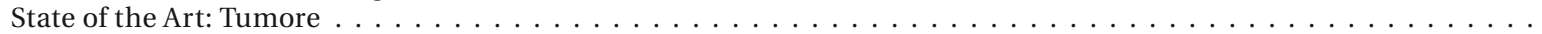

Schlussworte des Präsidenten und Übergabe der Amtsgeschäfte $\ldots \ldots \ldots \ldots \ldots \ldots \ldots \ldots \ldots \ldots$

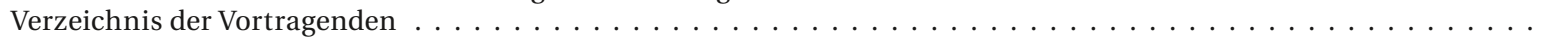

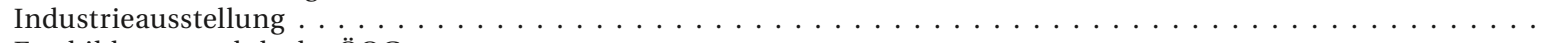

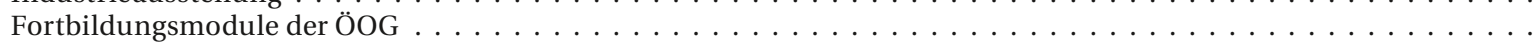

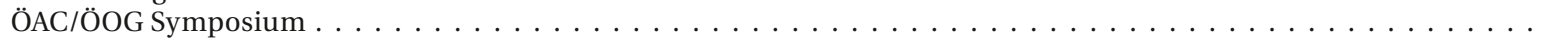

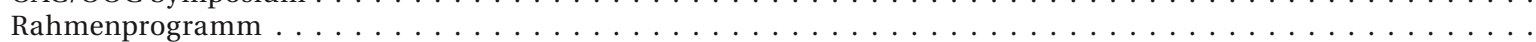

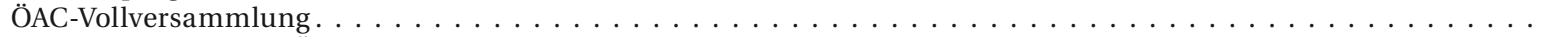

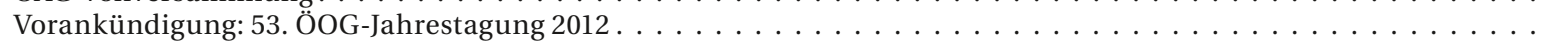

Impressum 

administratives Programm

Mittwoch, 1. Juni 2011

Sitzung des Beirates des Adele-Rabensteiner-Preises 16:00 Uhr Hotel Neuhaus

Vorstandssitzung

17:00 Uhr

Hotel Neuhaus

16.00 Uhr

Modul Cataract

Europahaus, Saal Kellertheater

Modul „Medizinrecht/Gutachten“

18.45 Uhr

Europahaus, Saal Kellertheater

Diagnostisches Konsil

A-ARVO - H-ARVO Science Day

Eröffnung der Jahrestagung 2011

1. Wissenschaftliche Sitzung

\section{Wissenschaftliche Sitzung}

Vollversammlung der Österreichischen Ophthalmologischen Gesellschaft

Primarärztesitzung

Hotel Neuhaus

\section{Wissenschaftliche Sitzung}

\section{Posterpräsentation}

Verleihung der Posterpreise

\section{Wissenschaftliche Sitzung} „State-of-the-art": Tumore des Auges

ÖAC/ÖOG-Symposium

„Wirtschaftliche und praktische Aspekte in der Augenheilkunde“

Europahaus, Saal Kellertheater

Vollversammlung ÖAC

Europahaus, Saal Kellertheater

Die oben genannten Veranstaltungen finden im Europahaus, Saal Europa statt.
Donnerstag, 2. Juni 2011

08:00 Uhr

10:00 Uhr

13:15 Uhr

13:30 Uhr

14:00 Uhr

Stadtrundgang, Erlebnissennerei

18:30 Uhr

Gesellschaftsabend mit der Industrie

Freitag, 3. Juni 2011

06:30 Uhr

2. ÖOG Lauf für Alle

Start: Europahaus

08.00 Uhr

$10.00 \mathrm{Uhr}$

Ausflug: Schloss Tratzberg, Silberbergwerk

$10.30 \mathrm{Uhr}$

14:00 Uhr

15.00 Uhr

$20.00 \mathrm{Uhr}$

Präsidentenempfang (separate Einladung)

Samstag, 4. Juni 2011

$08.00 \mathrm{Uhr}$

$09.50 \mathrm{Uhr}$

10:00 Uhr

$13.30 \mathrm{Uhr}$

17:00 Uhr

Programmänderungen vorbehalten Zeitangaben s. t.

Die Anmeldung zu den gesellschaftlichen Veranstaltungen ist mit dem beiliegenden Fax-Formular erbeten bzw. Online über unsere Website 


\section{Grußwort}

Sehr geehrte Kolleginnen und Kollegen!

Zur 52. Tagung der Österreichischen Ophthalmologischen Gesellschaft, welche vom 2.-4. Juni 2011 in Mayrhofen im Zillertal stattfindet, darf ich Sie auf das herzlichste begrüßen.

Der Veranstaltungsort Mayrhofen wird uns ein sehr angenehmes Ambiente bieten, um eine sehr interessante und wiederum auch sportliche Tagung (vielen Dank an Prof. Kieselbach für die Organisation des 2. ÖOG-Laufes) zu gestalten.

Ziel unseres jährlichen Kongresses ist an erster Stelle der Wissens- und Erfahrungsaustausch, sowohl neue wissenschaftliche Erkenntnisse mit Praxisrelevanz, als auch wesentliche standespolitische Themen betreffend. Deswegen möchte ich Sie an dieser Stelle auch vielmals um Ihre zuverlässige Teilnahme an der Vollversammlung ersuchen.

Wiederum wird ein „Diagnostisches Konsil“ gestaltet, welches vor dem „A-ARVO - H-ARVO Science Day“ am Donnerstag Morgen stattfinden wird. Junge Kolleginnen und Kollegen aus Kliniken und Abteilungen sollen interessante - und gegebenenfalls auch noch nicht vollständig geklärte - Fallbeispiele präsentieren. Sie werden dann von einer Runde von Diskutanten unter der Moderation von Kollegen Priglinger und Beteiligung der Zuhörer analysiert werden.

Am erstmals stattfindenden A-ARVO - H-ARVO Science Day werden Sie erneut einen Überblick über die neuesten Arbeiten junger ungarischer und österreichischer Wissenschaftler bekommen; dieser Teil des Programmes wird von Frau Prof. Binder organisiert und geleitet.

Darüber hinaus freuen wir uns sehr, dass es wiederum gelungen ist, aus den zahlreichen angemeldeten Beiträgen ein sehr breit gefächertes Programm zu erstellen, welches für jede Ophthalmologin und jeden Ophthalmologen von Interesse sein dürfte. Als „State of the Art“ Thema wurde diesmal „Tumore in der Ophthalmologie“ gewählt.

Mein großer Dank gilt auch der Unterstützung durch die Industrie. Wir schätzen diese gute Zusammenarbeit über viele Jahre und den laufenden Dialog über weitere gemeinsame Projekte und neue Produkte für unser Fach sehr!

Wie bei jeder internationalen Tagung üblich ist, erinnern wir daran, dass auch bei diesem Kongress jeder Sprecher verpflichtet ist, ein allfälliges finanzielles Interesse aller Autoren am zweiten Dia des Vortrages oder am Poster (in einer eigenen Box) bekannt zu geben, um Zweifel schon im Vorfeld auszuschließen.

Ganz besonders freuen wir uns über die gesponserten Preise, welche auf dieser Jahrestagung vergeben werden:

- A-ARVO: 1. Fa. Alcon: $€ 2.500$,- für eine Reise zur amerikanischen ARVO des darauffolgenden Jahres,

2. Fa. MSD: € 700,- für einer Reise zur EVER,

3. Fa. Novartis $€ 700$,- für eine Reise zur EVER,

- $\quad$ Kataraktsitzung: Fa. Zeiss: $€ 1.500$,- für einen Wet-lab Besuch für den besten Vortrag dieser Sitzung,

- Springer Preis - „Spektrum der Augenheilkunde“: € 2.000,- für die beste Publikation eines/-r unter 40-jährigen Autors/In im Jahr 2010,

- den MR Prim. Prof. Dr. Paul Drobec-Preis für das „beste Poster“ (inhaltlich und in seiner Gestaltung).

Ich darf mich im Namen des Vorstandes der ÖOG bei allen Mitarbeiterinnen und Mitarbeitern, welche zum Gelingen dieser Tagung schon vieles beigetragen haben, aufrichtig bedanken und wünsche Ihnen einige interessante und vielleicht auch erholsame Tage in Mayrhofen im Zillertal.

Mit herzlichen kollegialen Grüßen

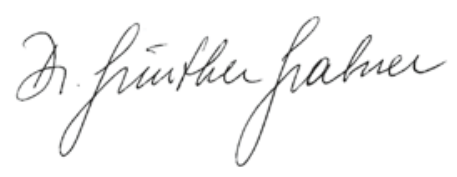

Ihr Prim. Univ.-Prof. Dr. Günther Grabner Präsident der ÖOG

Zugunsten einer besseren Lesbarkeit wurde auf eine geschlechtsneutrale Schreibweise meist verzichtet. 


\section{Vollversammlung der Österreichischen Ophthalmologischen Gesellschaft}

Ort: Europahaus, Saal Europa

Zeit: Freitag, 3. Juni 2011, 10:30 Uhr

Tagesordnung:

1. Begrüßung und Bericht des Präsidenten

2. Bericht der ständigen wissenschaftlichen Sekretärin

- Adele-Rabensteiner-Preis

- Strategien der Öffentlichkeitsarbeit

3. Bericht des Vorsitzenden des Fachgruppenbeirates

4. Bericht der Bundesfachgruppenobfrau

5. Bericht des Schriftführers

6. Aufnahme neuer Mitglieder

7. Rechnungsbericht des Kassiers und Festsetzung des Mitgliedsbeitrages

8. Entlastung des Kassiers

9. Kommissionsberichte

Einleitung durch die ständige wissenschaftliche Sekretärin und Berichte durch die Vorsitzenden der Kommissionen

10. Anträge

11. Allfälliges

Anträge an die Vollversammlung sind dem Vorstand bis spätestens 14 Tage vor der Vollversammlung schriftlich an das Sekretariat der ÖOG bekanntzugeben.

Prim. Univ.-Prof. Dr. Günther Grabner Präsident

Priv.-Doz. Dr. Matthias Bolz

Schriftführer
Univ.-Prof. Dr. Susanne Binder Ständ. wiss. Sekretärin

Univ.-Prof. Dr. Michael Georgopoulos stv. Schriftführer 


\section{Hinweise für Kongressteilnehmer}

Die Veranstaltungen, Vorträge, Posterdemonstrationen, die Vollversammlung und die Industrieausstellung finden im Europahaus Mayrhofen statt:

Europahaus Mayrhofen

Zillertal Congress

Dursterstraße 225

A-6290 Mayrhofen

Tel. 0043 (0)5285-6750

Homepage: www.europahaus.at

e-mail: congress@europahaus.at

Telefon: Kongresssekretariat vom 1. Juni bis 4. Juni 2011:

+43-69914028540

\section{Anreise}

Mit dem Auto

über die Inntalautobahn bis zur Ausfahrt Zillertal und von dort noch 30 km auf der Zillertal Straße Nr. 169 bis nach Mayrhofen.

\section{Mit dem Bus}

bis Bahnhof Jenbach (Schnellzugstation) und von dort noch $35 \mathrm{~km}$ mit dem Bus der Zillertaler Verkehrsbetriebe nach Mayrhofen.

\section{Mit dem Zug}

bis Bahnhof Jenbach (Schnellzugstation) und von dort noch 35 km mit der Zillertalbahn der Zillertaler Verkehrsbetriebe nach Mayrhofen.

\section{Mit dem Flugzeug}

Flughafen München/Franz-Josef-Strauß 190 km

Flughafen Salzburg/Maxglan 170 km

Flughafen Innsbruck $65 \mathrm{~km}$

Weitere Informationen zur Anreise unter: www.mayrhofen/ferienregion/anreise.html

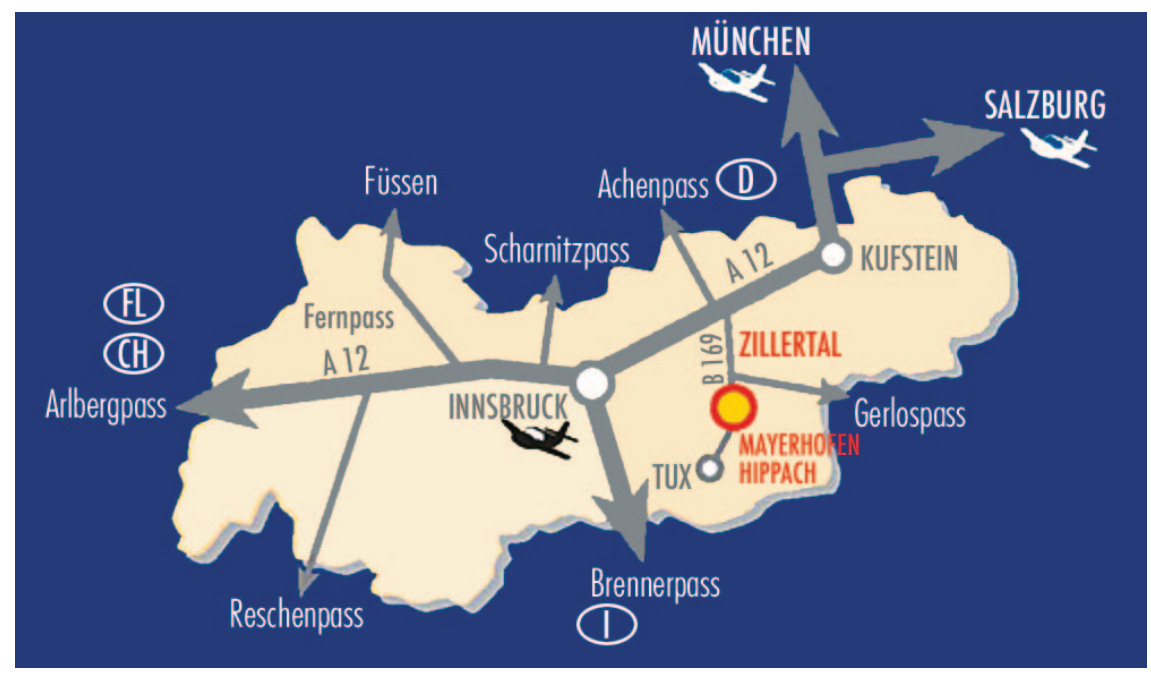




\section{Tagungsgebühr}

Die Tagungsgebühren sind bei Mitgliedern der ÖOG im jährlichen Mitgliedsbeitrag eingeschlossen.

Für Nichtmitglieder der ÖOG beträgt die Gebühr € 120,-; für Kollegen in Ausbildung (Bestätigung erforderlich)

$€$ 60,-; für Orthoptisten/innen $€ 30$,-.

Die Tagungsgebühr wird auch von jenen Vortragenden eingehoben, die nicht Mitglieder der ÖOG sind.

Die Tagungsgebühr und eventuell offene Mitgliedsbeiträge sind vor Ort in BAR zu begleichen (Bankomat, etc. ist technisch leider nicht möglich)!

\section{Diplomfortbildungsprogramm}

Vor Jahren ist von der Österreichischen Ärztekammer das Diplomfortbildungsprogramm eingeführt worden. Für diese Fortbildungen werden für die gesamte Tagung 23 Fortbildungsstunden gutgeschrieben.

Die Teilnahmebestätigungen, die dann für alle drei Jahre stattfindende Einreichung bei der Ärztekammer Gültigkeit haben, erhalten Sie direkt vor Ort.

\section{Mitgliedschaft bei der ÖOG}

Kolleginnen und Kollegen, die Mitglieder der ÖOG werden möchten, werden gebeten ihre Anfrage an das Sekretariat der ÖOG, Schlösselgasse 9/4, A-1080 Wien, zu richten. Sie erhalten dann das entsprechende Anmeldeformular und werden ab diesem Zeitpunkt in der Adresskartei geführt.

Die formale Aufnahme erfolgt jeweils bei der Vollversammlung der ÖOG.

Der Mitgliedsbeitrag beträgt jährlich für KollegInnen in Ausbildung € 70,-, für KollegInnen an Kliniken oder Augenabteilungen ohne Ordination $€ 140$,-, für KollegInnen mit Ordination $€ 210$,-, sowie für ausländische Kollegen $€ 130$,-Im Mitgliedsbeitrag ist die Teilnehmergebühr für die Jahrestagung der ÖOG, sowie der kostenlose Besuch für KollegInnen in Ausbildung einzelner Fortbildungsveranstaltungen enthalten.

Zahlende Mitglieder der ÖOG erhalten ohne zusätzliche Kosten die Zeitschrift Spektrum der Augenheilkunde sechs mal jährlich mit den Publikationen der wissenschaftlichen Tagungsbeiträge sowie der Gesellschaftsberichte, weiters die Reihe der Informationsbroschüren für Patienten über die wichtigsten Augenerkrankungen.

\section{Zahlung der Mitgliedsbeiträge}

Die Versendung der Zahlscheine für die jährlichen Mitgliedsbeiträge ist im Jänner d. J. bereits erfolgt. Bitte veranlassen Sie die Überweisung der fälligen Mitgliedsbeiträge sowie etwaiger Außenstände auf das PSK-Konto Nr. 7744534, BLZ 60000.

Bei Auslandsüberweisungen achten Sie bitte darauf, dass Bankspesen zu Lasten des Einzahlers gehen. Offene Mitgliedsbeiträge können während der Tagung am Schalter des Tagungsbüros in BAR beglichen werden, um den kostenlosen Besuch der Tagung zu ermöglichen bzw. bei der Vollversammlung stimmberechtigt zu sein.

\section{Anmeldung zur Tagung und zum Rahmenprogramm}

Für die Anmeldung zum Rahmenprogramm verwenden Sie bitte das beiliegende Fax-Formular, kreuzen Sie die gewünschten Veranstaltungen an, vermerken Sie die Anzahl der Teilnehmer, tragen Ihre Anschrift ein und übersenden es unterschrieben an +43-1-402 7935.

Die Anmeldung soll so bald als möglich erfolgen, um einen reibungslosen Ablauf des Programmes zu ermöglichen. Die Plätze für das Rahmenprogramm werden in der Reihenfolge der eingelangten Bestellungen reserviert. Bei zu geringer Beteiligung können Veranstaltungen abgesagt werden.

Sollten Sie das Anmelde-Fax-Formular noch nicht erhalten haben, können Sie es direkt unter Tel. +43-1-402 85 40, bzw. e-mail: oeog@augen.at anfordern.

Ab diesem Jahr läuft die Anmeldung auch online über das neue Mitgliederverwaltungssystem der ÖOG. Melden Sie sich gleich jetzt für die Teilnahme an der Tagung, dem Modul, dem ÖAC Symposium, dem ÖOG Lauf oder dem Rahmenprogramm über unsere Homepage www.augen.at an.

Hinweis: Die Registrierung ist für alle Teilnehmer erforderlich, auch für Vortragende. Sollten Sie sich bereits mit Hilfe des Faxformulars für die Tagung oder andere Veranstaltungen angemeldet haben, ist eine neuerliche Anmeldung über dieses System NICHT erforderlich.

\section{Quartierbestellungen}

Richten Sie bitten an Christophorus Reisen

Tel. +43/5285-63200-101

e-mail: carmen@christophorus.co.at 


\section{Tagungsbüro}

Das Tagungsbüro befindet sich in der Eingangsebene im Europahaus.

Öffnungszeiten: Mittwoch, 1. Juni $2011 \quad 08.00-20.00 \mathrm{Uhr}$

Donnerstag, 2. Juni $2011 \quad 07.00-18.30 \mathrm{Uhr}$

Freitag, 3. Juni $2011 \quad 08.00-19.00 \mathrm{Uhr}$

Samstag, 4. Juni $2011 \quad 08.00-18.00 \mathrm{Uhr}$

Tel. +43-69914028540

\section{Wissenschaftliche Ausstellung - Posterpräsentation}

Für die wissenschaftliche Ausstellung steht der Vorbereich des Saal Europa zur Verfügung.

Die Poster sollen ab Donnerstag, 2. Juni 2011, 10 Uhr aufgehängt sein und während der gesamten Tagung besichtigt werden können (Posterflächen 140 x 90 cm, Hochformat). Am Samstag, dem 4. Juni 2011, findet um 8 Uhr vor den Posterwänden eine Posterpräsentation statt. Die anwesenden Autoren werden unter Moderation ihre Arbeiten erläutern und stehen für Anfragen zur Verfügung. Wir ersuchen die Autoren dringend um rechtzeitige Montage der Poster.

Am Ende der Posterpräsentation findet ein Sektempfang statt. Die Überreichung des „Prim. Prof. Dr. Paul Drobec Posterpreises“ erfolgt zu Beginn der 4. Wissenschaftlichen Sitzung.

\section{Industrieausstellung}

Die umfangreiche Industrieausstellung findet in den Foyers statt. Die Ausstellung ist während der gesamten Tagungsdauer geöffnet.

Ausstellerverzeichnis und Plan auf den Seiten 197-199.

\section{Anfragen}

Richten Sie bitte an die

Österreichische Ophthalmologische Gesellschaft

Schlösselgasse $9 / 4$

A-1080 Wien

Tel. +43-1-402 8540

Fax +43-1-402 7935

Mobil +43 (0) 69914028540

Homepage: www.augen.at

e-mail: oeog@augen.at 


\section{Hinweise für die Vortragenden}

Redezeit

Die Redezeit für wissenschaftliche Beiträge beträgt:

Vorträge inkl. Diskussionbemerkungen . . . . . 6 + 2 Minuten

Kurzvorträge ohne Diskussion . . . . . . . 4 Minuten

Es widerspricht den Grundsätzen der Vereinigung, Vorträge zu halten, die schon vorher publiziert oder anderwärts vorgetragen wurden.

\section{Projektion}

PC-Projektion - bitte USB-Stick oder CDs mitbringen! Apple-User mit eigenem Notebook!

\section{Publikation}

Wissenschaftliche Beiträge, die vom Programmkomitee der ÖOG angenommen wurden, sind in publikationsfertiger Fassung am Ende des Jahreskongresses, spätestens aber bis zum 30. 6. 2011 zur Publikation im „SPEKTRUM DER AUGENHEILKUNDE“ einzureichen.

Den Autoren wird empfohlen, sich an die Hinweise für Autoren zu halten (online unter www.springer.com/717).

Für Vorträge und Demonstrationen, die auf der Jahrestagung der ÖOG gehalten worden sind, besitzt die Gesellschaft das Copyright. Das Nichteinreichen der Manuskripte zieht bei der Bewertung der Beiträge für die nächstjährige Tagung einen automatischen Punkteabzug nach sich. 


\title{
Donnerstag, 2. Juni 2011
}

08:00

\author{
DIAGNOSTISCHES KONSIL \\ Europahaus, Saal Europa \\ Einführung: G. Grabner \\ Moderation: S. Priglinger
}

10:00

\author{
A-ARVO - H-ARVO SCIENCE DAY \\ Europahaus, Saal Europa \\ Moderation: L. Schmetterer \\ Vorsitz: J. Nemeth, S. Binder
}

10:05

Lecture 1. B. Bogner, A. Trost, F. Schroedl, C. Strohmaier, C. Runge, G. Grabner, H. Reitsamer (Salzburg): Is there a link between aquaporin expression and intra ocular pressure regulation? Gibt es einen Zusammenhang zwischen Aquaporin-Expression und Augendruckregulation?

Purpose: Reducing intraocular pressure (IOP) is the most effective strategy to slow down the progression of glaucoma. Therefore, the knowledge of IOP formation and regulation is of great interest for glaucoma treatment. Many anti-glaucomatous drugs lower IOP by decreasing aqueous humor production, by increasing aqueous humor outflow, or both. However, ongoing research efforts investigate the molecular mechanisms and signaling pathways involved in IOP regulation. Here, the current knowledge about water channels, known as aquaporins (AQPs), which are crucial for water movements across membrane barriers and are expressed in many different ocular cell types, will be presented.

Methods and/or patients: The distribution of AQPs (AQP0, AQP1, AQP2, AQP3, AQP4 and AQP5) in the rabbit eye was investigated by immunohistochemistry and western blot analysis. The ocular expression patterns of AQPs in rabbit will be compared with published data for human, mouse and rat. Functional studies in mice will be summarized.

Results: The distribution of AQP4 in the rabbit eye is similar to the expression patterns in human, mouse and rat. Immunohistochemistry of AQP0, AQP1, AQP2, AQP3 and AQP5 is still under investigation. Due to the topography of AQP1 (lens epithelium, corneal endothelium, trabecular meshwork, ciliary epithelium and keratocytes) and AQP4 (ciliary epithelium, optic nerve and müller cells), these water channels are presumed candidates for IOP regulation. The functional contribution of AQPs to aqueous secretion in vivo was demonstrated in mice lacking AQP1, AQP4 or both. IOP in knock-out mice for AQP1-/-, AQP4-/-, or AQP1-/-/ AQP4-/- was significantly lower than in wild-type mice.

Conlusions: The present results suggest that AQP1 and AQP4 are involved in IOP regulation. Functional studies have shown that the reduction in IOP is caused at least partly by lower levels of aqueous humor production. However, at this time the role of AQPs in IOP regulation is not defined and the possibilities for glaucoma treatment remains speculative. Functional studies in the acute rabbit model may contribute to identify the role of AQPs in IOP regulation.

Schlüsselwörter: Aquaporine, Augendruckregulation.

Key words: Aquaporins, intraocular pressure regulation.

10:11

Lecture 2. A. Trost, F. Schroedl, B. Bogner, C. Strohmaier, G. Grabner, L. Aigner, H. Reitsamer (Salzburg): Comparative characterization of choroidal and retinal pericytes - Charakterisierung choroidaler und retinaler Perizyten 
Purpose: Pericytes, cells closely adjacent to blood vessels, are important for vascular development, stabilization and remodelling. They potentially influence vascular diameter. In pathologic conditions (e.g., diabetes, hypertension, neovascularization) a change in pericyte coverage of microvessels is found, suggesting a possible key role of pericytes in the pathogenesis of various blood flow related diseases. However, the function of pericytes in these ocular circuits is not completely understood. In the present study, we present a thorough characterization of retinal and choroidal pericytes as basis for pending functional studies.

Methods and/of patients: Using immunohistochemistry, cultivated bovine retinal and choroidal pericytes were tested for the pericyte markers NG2, CD146, PDGFRb, $\alpha$-SMA. To distinguish pericytes from endothelial cells in vitro as well as in retinal and choroidal cryosections, a screening for the endothelial cell markers CD31 and lectin1 was performed. Additionally, real-time PCR was used to identify pericyte and endothelial marker expression on mRNA level.

Results: Immunohistochemistry revealed a co-localization of the pericyte markers NG2 and CD146/PDGFRb in tissue slices and cultivated retinal and choroidal perivascular cells. All cultivated pericytes were $\alpha$-SMA-positive, whereas in cryosections only pericytes surrounding vessels $>10 \mu \mathrm{m}$ showed immunoreactivity for $\alpha$-SMA. The endothel specific marker lectinl was co-localized with NG2, CD146 and $\alpha$-SMA. On mRNA expression level NG2 was expressed by pericytes only, while CD146 and $\alpha$-SMA were also detectable in endothelial cells.

Conclusions: Although data on pericyte characterization have been released, no distinct pericyte marker is defined up to now. Pericyte markers tested here partly showed an overlap with endothelial markers and vice versa. Our study highlights the challenge to find an explicit pericyte marker and also the importance to differentiate between pericytes and endothelial cells independently of their characteristic position in and around vessels. Additional characterization studies are currently performed to unveil a proper marker combination in order to definitely identify pericytes as a basis for functional studies.

Schlüsselwörter: Retinale und choroidale Perizyten, Perizytenmarker, Endothelzellmarker, Protein- und Genexpression.

Key words: Retinal and choroidal pericytes, pericyte marker, endothelial cell marker, gene and protein expression.

Lecture 3. H. Reitsamer, A. Trost, B. Bogner, C. Strohmaier, L. Aigner, C. Runge, F. Schrödl (Salzburg): Pericytes may play a role in choroidal blood flow regulation - first evidence - Mögliche Rolle von Perizyten in der Durchblutungsregulation der Aderhaut - Erste Hinweise

Purpose: For ocular blood flow homeostasis, adjustment of the vessel diameter is the most crucial point. This diameter is regulated via extrinsic pathways involving the autonomic nervous system as well as intrinsic humoral and paracrine factors, involving e.g., nitric oxide and endothelin but also oxygen and $\mathrm{CO}_{2}$. The intrinsic humoral regulation is poorly understood, and the mechanisms of neurovascular coupling remain unclear even more in the retina than in the choroid. Pericytes are cells closely surrounding blood vessels. Pericytes in ocular tissues are poorly understood and might represent an important source for blood flow regulation under various aspects.

Methods and/or patients: Choroidal and retinal wholemounts of Sprague Dolly rats were cryosectioned, followed by single- and double-immunhistochemistry against PGP9.5, NG2, and CD 146. For documentation, confocal laser-scanning microscopy and fluorescence microscopy was used.

Results: In retinal wholemounts, around 4 to 7 pericytes per $100 \mu^{2}$ were counted. The average cell diameters reached $5-10 \mu \mathrm{m}$, with processes traceable up to $20 \mu \mathrm{m}$. In rat choroidal wholemounts, 7 to 10 pericytes $/ 100 \mu \mathrm{m}^{2}$ were counted. Choroidal pericytes reached average cell diameters of $10 \times 10 \mu \mathrm{m}$ with processes sometimes reaching up to $80 \mu \mathrm{m}$ at estimated vessel diameters of $20 \mu \mathrm{m}$. Processes of pericytes in both ocular tissues surround blood vessels and were detected to co-localize the pericyte markers NG2 and CD146. PGP 9.5 positive pro- 
files were found in close apposition to pericytes, suggesting innervation of the pericyte system by the autonomic nervous system.

Conclusions: The massive layer of pericytes around the choroidal vasculature as well as the innervation of pericytes with PGP9.5 positive processes suggests a role of the pericyte system in autonomic choroidal blood flow regulation. The extent of engulfment outreaches what has been reported for the retinal circulation, however, as to whether choroidal pericytes are involved into autoregulatory mechanisms of the choroid remains to be investigated.

Schlüsselwörter: Aderhaut, Perizyten, Durchblutungsregulation.

Key words: Choroid, pericytes, blood flow regulation.

Lecture 4. C. Strohmaier, D. Link, B. Seiffert, G. Grabner, H. Reitsamer (Salzburg): Retinal vessel diameter analysis in the rat: First functional results - Retinale Gefäßanalyse in Ratten: Erste funktionelle Ergebnisse

Purpose: To evaluate a novel retinal vessel analyzer for rats. Retinal vessels lack innervations and their diameter is controlled by local factors like oxygen and nitric oxide only. Oxygen and the nitric oxide synthase inhibitor L-NAME have well-known effects on the retinal arterial and venous diameter in other species. In rats, continuous, real time measurements of the retinal vessel diameters were not possible in the past due to a lack of appropriate instruments. Here, we present a novel instrument for retinal vessel analysis and use well known vasoactive agents for functional testing.

Methods and/or patients: : Anesthetised Male Brown-Norway rats $(n=12)$ were tracheotomized respired with room air. The ventilation parameters were chosen to keep the expired $\mathrm{CO}_{2}$ in at $40 \mathrm{mmHg}$. A catheter for blood pressure measurement was inserted into the right femoral artery and advanced to the aorta, another catheter was inserted into the right femoral vein for drug administration. Sodium hyaluronate eye drops were used to keep a stable tear film and the cornea moisture. The retinal vessel diameters were measured with a custom-made, non-contact fundus camera and the Imedos DVA software tool. This setup enables a continuous measurement of the retinal vessel diameters. After a 1 minute baseline measurement, $100 \%$ oxygen or L-NAME i.v. $(5 \mathrm{mg} / \mathrm{kg})$ were given and the vessel diameters werenrecorded over a period of 6 minutes.

Results: The mean arterial blood pressure was $98.91 \mathrm{mmHg}$ (oxygen group) and $87.34 \mathrm{mmHg}$ (L-NAME group) before the measurements. Oxygen inhalation increased the blood pressure by $12 \%$ after 6 minutes, it decreased the arterial vessel diameter by $13 \%$ and the venous diameter by $6 \%$. L-NAME application increased the blood pressure by $45 \%$, it decreased the arterial vessel diameter by $7 \%$ and the venous diameter by $2 \%$.

Conclusions: This is the first continuous measurement of retinal vessel diameter in rats. The effects of oxygen inhalation and L-NAME are comparable to the effects published in the literature. The instrument presented enables the use of rat models for investigations of the role of retinal vessel diameter in ocular diseases. Schlüsselwörter: Netzhaut, Gefäßanalyse, Ratte.

Key words: Retina, vessel analysis, rats.

$10: 35$

Lecture 5. F. Schrödl, C. Strohmaier, B. Bogner, A. Trost, G. Grabner, D. Nickla, H. Reitsamer (Salzburg): Neuronal control of choroidal accommodation: a morphological perspective - Neuronale Kontrolle choroidaler Akkommodation: ein morphologischer Blickwinkel

Purpose: The choroid is supplied with a dense sympathetic, and primary afferent (sensory) innervation. Besides this extrinsic innervation, some species possess an intrinsic choroidal innervation, mediated by ganglia and neurons within the
Bemerkungen 
choroid, the intrinsic choroidal neurons (ICN's). Targets of this intrinsic innervation are vascular and non-vascular smooth muscle cells. Here, we summarize the data obtained so far in birds with focus on the chicken, discuss their implementation in extrinsic circuits of the autonomic nervous system (mainly the pterygopaltine ganglion, PPG) and their impact on choroidal accommodation.

Methods and/or patients: Up to date, immunohistochemistry and cytochemistry at the light- and ultrastructural level only reveals answers about ICN, any functional data are still lacking.

Results: Immunohistochemistry revealed that ICN's are apposed by sympathetic, parasympathetic and also primary afferent nerve fibers, and possibly also receive contacts from other intrinsic neurons. The majority of avian ICN's are positive for vasoactive intestinal polypeptide (VIP), galanin (GAL) and neuronal nitric oxide (nNOS), but lack choline acetyl-transferase and somatostatin. A similar chemical coding (VIP/GAL/nNOS) is found in $50 \%$ of the neurons in the PPG. Lesion studies on the PPG pathway, however, revealed no influence on compensatory response to myopic defocus. A role for the PPG in these responses however cannot be excluded because of possible sympathetic and parasympathetic interganglionic pathways.

Conclusions: The innervation pattern in and especially to the avian choroid is more complex than expected. The neuronal control of choroidal thickness (via blood vessels or non-vascular smooth muscle cells) still is an equation with too many unknown parameters.

Schlüsselwörter: Autonomes Nervensystem, experimentelle Myopie, Stickstoffmonoxid.

Key words: Autonomic nervous system, experimental myopia, nitric oxide.

$10: 41$

Lecture 6. C. Runge, B. Bogner, H. Brandtner, C. Strohmaier, F. Schrödl, G. Grabner, H. Reitsamer (Salzburg): The effect of PACAP on choroidal blood flow regulation in anesthetized rabbits - Einfluss von PACAP auf die choroidale Durchblutungsregulation in anästhesierten Kaninchen

Purpose: To investigate the effect of PACAP (PACAP-27) and its antagonist PAC AP 6-38 on choroidal blood flow regulation in anesthetized rabbits.

Methods and/or patients: In anesthetized New Zealand white rabbits ( $n=9)$ mean arterial pressure (MAP) and intraocular pressure (IOP) were measured with pressure transducers by direct cannulation of the central ear artery, and the vitreous, respectively. Laser Doppler flowmetry was used to measure choroidal blood flow (ChorBF) and choroidal vascular resistance (ChorR) was calculated. PACAP-27 infusion was administrated intravenously $(20 \mathrm{ng} / \mathrm{kg} / \mathrm{min})$ with an infusionrate of $2 \mathrm{~mL} / \mathrm{h}$. PACAP $6-38$ was given as a bolus in a concentration of $40 \mathrm{ng} / \mathrm{kg}$ body weight. Ocular perfusion pressure was manipulated mechanically with hydrolic occluders around the large vessels and all variables were recorded continuously.

Results: baseline changes could be recognized in response to PACAP-27 and PACAP 6-38 on ChorBF, IOP, MAP, ChorR and PP (perfusion pressure). The increase of ChorBF and decrease of ChorR after PACAP-27 infusion was significant as it was on the increase of IOP. The administration of its antagonist PACAP 6-38 was able to block the effects of IOP, ChorBF and ChorR. In addition to the basline changes choroidal pressure flow relationships were shifted upwards by PACAP-27 indicating vasdilation over a wide range of perfusion pressures. The shift was reversed by PACAP 6-38. The effects on ChorBF as well as on ChorR were statistically significant.

Conclusions: PACAP is a high potent vasodilator in the choroidal vasculature. The vasodilatory effect was blocked by the receptor antagonist PACAP 6-38.

Schlüsselwörter: Choroidea, Durchblutungsregulation.

Key words: Choroid, blood flow, drug effects. 
Lecture 7. H. Brandtner, C. Runge, C. Strohmaier, B. Bogner, F. Schrödl, G. Grabner, H. Reitsamer (Salzburg): Central nervous stimulation: The effect of L-NAME on the stimulation-response curve - Zentralnervöse Stimulation: Der Effekt von L-NAME auf die Stimulation-Response Kurve

Purpose: To investigate the frequency-dependent effect of facial nerve stimulation on choroidal blood flow. In contrast to our previous stimulation protocols we put the focus on different frequencies as the resulting stimulation-response curve seems to be a powerful instrument for further investigations on the neurovascular coupling in the eye. In addition, the effect of L-NAME on the frequency dependence of the effect of facial nerve stimulation on choroidal blood flow was investigated in the present study.

Methods and/or patients: Mean arterial pressure (MAP), intraocular pressure (IOP) and choroidal blood flow (ChorBF) were measured in anesthetized new Zealand white rabbits $(\mathrm{n}=4)$. MAP and IOP were measured invasively and Laser Doppler Flowmetry was used to measure choroidal blood flow. The facial nerve, which carries parasympathetic fibers to the ciliary ganglion, was exposed in the middle ear cavity using an approach through the tympanic membrane. A platinum electrode was used to apply pulse trains between 2 and $20 \mathrm{~Hz}(200 \mu \mathrm{s}, 2.5 \mathrm{~mA}$, total duration of $20 \mathrm{~s}$ ) before and after application of the non selective NOS inhibitor, L-NAME (N- $\omega$-Nitro-L-Arginine-methylester, $128 \mathrm{mg} / \mathrm{kg}$, i.v. bolus).

Results: There is a direct correlation between frequency and response. With increasing frequency the IOP and the ChorBF increase. The use of the L-NAME shifts the stimulation-response curve for ChorBF as well as for IOP downwards, but as previously shown it cannot block the stimulation response completely, on the contrary, the effect is smaller than expected from other reports in the scientific literature.

Conclusions: As the stimulation-response curves illustrates the effect of L-NAME it seems to be a useful tool to investigate the effect of antagonists of other players in neurovascular coupling of the eye like vasoactive intestinal peptide (VIP) or pituitary adenylate cyclase-activating polypeptide (PACAP).

Schlüsselwörter: Choroidaler Blutfluss, IOD, autonome Innervation.

Key words: Chroidal blood flow, IOP, autonomic innervation.

Lecture 8. C. Strohmaier, B. Bogner, A. Trost, C. Runge, F. Schrödl, G. Grabner, H. Reitsamer (Salzburg): From lab books towards data mining: Electronic science documentation in at the Eye Clinic Salzburg and its european collaboration partners - Vom Laborbuch zum Data Mining: Wissenschaftsdokumentation an der Universitätsaugenklinik Salzburg

Purpose: Today's science relies heavily on information technology, but the documentation of scientific ideas, experiments and results is still done on paper - in individual lab books. This approach impairs international collaborations - which are necessary to address today's scientific challenges. Here, we present solutions to facilitate science do-cumentation on a single lab basis as well as on a European scale.

Methods and/or patients: All scientific processes of our multi-modal laboratory have been modeled in a relational data model. The data is accessible through a server-client architecture and is embedded in the clinic's IT system in order to achieve a high level of data security and a reliable service provision.

Results: The data model enables not only the documentation of experimental results, but also the documentation of the ideas and hypothesis that led to the conduction of the experiments. Furthermore, all links between those ideas, experiments and results can be documented and are fully traceable. The analysis afterwards can be performed under any desired aspect, e.g. all experiments can be analyzed by antibodies used or the different drugs that have been applied.

Conclusions: The system presented provides a modern way of science documen- 
tation. Especially the possibility of automated post-hoc analysis under any desired aspect provides an significant advantage over traditional book documentation. The used architecture allows for an easy an cost-efficient implementation of the documentation system in an existing IT-infrastructure. Furthermore, a concept for a European approach to support collaborations is presented.

Schlüsselwörter: Wissenschafts-Dokumentation.

Key words: Science documentation.

Lecture 9. K. Lorenz, J. Troger, O. Gramlich, R. Fischer-Colbrie, E. Schmid, G. Haas, N. Bechrakis (Mainz): PE-11, a peptide derived from chromogranin B, in the rat eye - PE-11 im Auge der Ratte

Purpose: The aim of the study was to investigate the presence and distribution of PE-11, a peptide derived from chromogranin B, in the rat eye.

Methods and/or patients: For this purpose, newborn rats were injected with a single dosage of $50 \mathrm{mg} / \mathrm{kg}$ capsaicin subcutaneously under the neck fold and after three months, particular eye tissues were dissected and the concentration of PE-11-like immunoreactivity was determined by radioimmunoassay. Furthermore, PE-11-like immunoreactivities were characterized in an extract of the rat eye by reversed phase HPLC. Then, the distribution pattern of PE-11 was investigated in the rat eye and rat trigeminal ganglion by immunofluorescence.

Results: As a result, PE-11 was present in each tissue of the rat eye and capsaicin pretreatment led to a $88.05 \%( \pm 7.07)$ and a $64.26 \%( \pm 14.17)$ decrease of the levels of PE-11 in the cornea and choroid/sclera, respectively, and to a complete loss in the iris/ciliary body complex. Approximately $70 \%$ of immunoreactivities detected by the PE-11 antiserum have been found to represent authentic PE-11. Sparse nerve fibers were visualized in the corneal and uveal stroma, surrounding blood vessels at the limbus, ciliary body and choroid and in association with the dilator and sphincter muscle. Furthermore, immunoreactivity was present in the corneal endothelium. In the retina and optic nerve, glia was labeled. In the rat trigeminal ganglion, PE-11-immunoreactivity was visualized in small and medium sized ganglion cells with a diameter of up to $30 \mu \mathrm{m}$.

Conclusions: In conclusion, there is unequivocal evidence that PE-11 is a constituent of capsaicin-sensitive sensory neurons innervating the rat eye and the distribution pattern is typically peptidergic in the peripheral innervation but in the retina completely atypical for neuropeptides and unique.

Schlüsselwörter: PE-11, Neuropeptid, Immunfluoreszenz, Ratte.

Key words: PE-11, neuropeptide, immunofluorescence, rat.

Lecture 10. G. Petrovski, A. Facskó, Z. Sarang, R. Albert, E. Johnsen, R. Frøen, B. Nicolaissen, M. Moe, A. Berta (Debrecen, Oslo): Activated neuroepithelial progenitor cells are present in the vitreous of mice with proliferative vitreoretinopathy

Purpose: Proliferative vitreoretinopathy (PVR) is an essential cause and complication of retinal diseases such as retinal detachment due to fibrous membranes formation and tractional forces. It has not been fully elucidated how this process is initiated, but secretion of inflammatory substances and migration of mesenchymal, glial and inflammatory cells into the corpus vitreum are known to be contributing factors. We hypothesized that PVR activate neuroepithelial stem/ progenitor cells from the ciliary margin at the periphery of the retina to migrate into the vitreous.

Methods: Female, 4-6 months-old, wild type mice were anesthetized with pentobarbital and received $1 \%$ procaine hydrochloride local anesthetic and tropicamide for iris dilatation as approved by the Animal Care Committee of the University of Debrecen. PVR was induced by dispase $(U / \mu l)$ injected intravitreally under microscopic control in the right eyes. Frozen sections were obtained 
after 1 and 2 weeks and vitreous samples were cultured in a stem cell-promoting medium for up to 3 passages. PVR was diagnosed by optical coherence tomography and cells were characterized by immunocytochemistry, RT-PCR and flow cytometry.

Results: Small sphere-like structures were observed in close vicinity to the ciliary margin as early as one week from the PVR induction. Following isolation and culture of the vitreous, spheres formed in 7 out of 12 samples. These spheres showed a characteristic population of cells positive for neuroepithelial progenitor markers such as nestin, GFAP, Tuj1 and CD133, and displayed a mixed neural and epithelial morphology.

Conclusions: Our results indicate that a population of immature neuroepithelial progenitor cells are activated and migrate into the vitreous of mice models of PVR. This opens possibilities for knock-out animal studies and novel inhibitor agents against PVR.

Key words: PVR, progenitor cells, neuroepithelial, OCT.

Lecture 11. I. Fejes, P. Kocsis, M. Janáky (Szeged): Interocular differences on testing the function of the central retina in normal subjects

Purpose: The aim of the study was to determine the normal side difference of the central retinal function by multifocal electroretinography (mfERG), pattern electroretinography (PERG) and pattern visual evoked potential (PVEP). These may have role in evaluation of the severity of a monocular macular pathology.

Patients and methods: MfERG, PERG and PVEP were recorded according to the standards of International Society for Clinical Electrophysiology of Vision. ERGs were lead via DTL electrode. The active PVEP electrode was placed $2.5 \mathrm{~cm}$ above the inion. MfERG was performed on 35 subjects with visual acuity of 1.0. Pupils were dilated for the examination. The amplitudes of the scalar products were averaged over five concentric retinal regions (rings). PERG and VEP were performed with normal pupil size on 77 subjects with visual acuity of 1.0. The percentage of the side differences was determined, as interocular difference divided by the higher amplitude value. Results are shown as mean \pm SD.

Results: No significant differences were found between the electrophysiologic parameters on grouping by right and left eyes, but the differences were significant on comparison of the higher and lower response amplitudes. The interocular amplitude differences were as follows: mfERG $21.55 \pm 12.72 \%$. PVEP P100 implicit time $1.5 \pm 1.32 \%$, N75P100 amplitude $11.93 \pm 9.11 \%$ and P100N135 amplitude $13.33 \pm 9.13 \%$. PERG P50 implicit time $1.93 \pm 2.05 \%$, N35P50 amplitude $12.99 \pm 9.55 \%$ and P50N95 amplitude $11.69 \pm 9.97 \%$.

Conclusions: Our results revealed significant interocular amplitude difference of mfERG, PERG and VEP, the electrophysiological methods testing the central retinal funcion and related visual pathway. Without calculating with these side differences, misinterpretation may occur in a monocular pathological process.

Key words: Multifocal electroretinography, pattern electroretinography, visual evoked potential, interocular difference.

Lecture 12. A. Garas, A. Papp, G. Holló (Budapest): Influence of age-related macular degeneration on glaucoma measurements made with the RTVue Fourier-domain OCT

Purpose: To evaluate the influence of age-related macular degeneration (AMD) on retinal nerve fiber layer thickness (RNFLT), optic disc and inner macular retinal thickness parameters measured with Fourier-domain optical tomography (RTVue-OCT) in non-glaucomatous eyes.

Methods: One non-glaucomatous eye of 79 Caucasian persons was imaged. This comprised 25 healthy eyes, 19 eyes with early/intermediate AMD (geographic atrophy excluded), 16 eyes with subfoveal choroidal neovascularisation (CNV) and 19 CNV eyes after intravitreal antiangiogenic treatment (CNV-anti-VEGF). 
Results: Compared to the age-matched controls, no difference in any RNFLT and optic disc parameter was seen for any AMD group. The average macular thickness parameters did not differ between the controls and the AMD groups (ANOVA, p > 0.05). In contrast, all pattern-based Ganglion Cell Complex (GCC) parameters were significantly higher (more abnormal) in the CNV and CNV-anti-VEGF group than in the control eyes (Mann-Whitney test, Bonferroni correction, $\mathrm{p}<0.001)$. For GCC Focal Loss Volume (FLV), the only pattern-based parameter classified by the software, the frequency of "borderline" and "outside normal limits" classifications was significantly greater in each AMD group than in the control group (Chi-square test, Bonferroni correction, $\mathrm{p} \leq 0.03$ ). Inner retinal segmentation errors occurred in $0 \%, 42.1 \%, 100 \%$ and $89.5 \%$ of the control, drusen, CNV and CNV-anti-VEGF eyes, respectively (Chi square test, $\mathrm{p}<0.001$ ).

Conclusions: In non-glaucomatous eyes, AMD significantly influences the pattern-based inner macular thickness parameters of the RTVue-OCT and the software-provided classification of GCC FLV, thus a detailed examination of the macula is necessary before an abnormal GCC parameter is considered to be a sign of glaucoma.

Key words: Glaucoma, RTVue Fourier-domain optical coherence tomography, age-related macular degeneration, macular thickness.

Lecture 13. I. Kovács, C. Luna, S. Quirce, M. C. Acosta, C. Belmonte, J. Gallar (Budapest, San Juan de Alicante): Increased activity of corneal sensory receptors in an experimental model of dry eye

Purpose: To evaluate the effect of decreased tear secretion on nerve impulse activity of corneal sensory receptors in an animal model of dry eye.

Methods: The main lacrimal gland was removed in 18 guinea pigs. One, two or four weeks after the surgery, electrical activity was recorded from individual nerve endings in the cornea and from nerve fibers in the back of the eye superfused in vitro at $34^{\circ} \mathrm{C}$. In cold thermosensitive nerve terminals, spontaneous activity at basal temperature, cold threshold and maximum frequency response during cooling pulses down to $20^{\circ} \mathrm{C}$ were analyzed. In corneal nociceptive units, mechanical threshold was measured with von Frey hairs, chemical sensitivity was tested with 30 s gas jets of $98 \% \mathrm{CO}_{2}$ in air.

Results: Tear secretion significantly decreased at every timepoint after surgery ( $\mathrm{p}<0.001)$. Both spontaneous activity and maximum cool response increased significantly at 4 weeks $(p<0.01)$. Temperature threshold was elevated from the first week $(\mathrm{p}<0.01)$. Spontaneous rate of corneal mechano- and polymodal nociceptors was slightly increased 1 week after the surgery, but not 1 month after. Mechanical threshold was significantly decreased 1 week after surgery for mechano-nociceptors but not for polymodals. Response of polymodal nociceptors to a noxious chemical stimulation was significantly increased at 1 and 4 weeks after surgery.

Conclusion: Surgical removal of the lacrimal gland affects neural activity of corneal sensory receptors by increasing spontaneous and response activity. This sensitization may contribute to dryness sensations in dry eye pathologies.

Key words: Dry eye, corneal nerves.

Lecture 14. K. Toth-Kovacs, Z. Pamer, O. Rideg, A. Kovacs, S. Fekete, Z. Biro, GL. Kovacs (Pecs): Association of Alzheimer's disease and age-related macular degeneration in South-Western Hungary

Purpose: The pathomechanisms of age-related macular degeneration (AMD) and Alzheimer dementia (AD) show several similarities (advanced age, formation of deposits of similar content). Allelic variations of apolipoprotein E (apoE) are associated with these diseases: apoE4 is associated with increased risk and earlier onset of $\mathrm{AD}$ whereas apoE2 with reduced susceptibility of $\mathrm{AD}$, but increased risk of AMD. Recently the AMD associated complement factor $\mathrm{H}(\mathrm{CFH})$ gene has also been shown to influence the risk of $\mathrm{AD}$. We investigated, therefore, the occurrence 
of AMD in AD patients and compared their lipid profile and apoE polymorphism. Methods and/or patients: $96 \mathrm{AMD}, 84 \mathrm{AD}$ and 35 control patients were examined (risk factors, visual acuity, slit lamp biomicroscopy, fundoscopy). Measurements of triglyceride, total- and HDL cholesterol levels, as well as the analysis of apoE alleles were performed.

Results: The prevalence of apoE 3 isoform (most commonly seen in the population) in the AMD, AD and the control patients was $80 \%, 53 \%$ and $71 \%$, respectively. The prevalence of the apoE 4 isoforms was $6 \%, 37 \%$ and $23 \%$, while that of apoE 2 was 14\%, 10\% and 6\%, respectively. Triglyceride, total and HDL cholesterol levels were in the reference range; however, Alzheimer patients tended to have a lower total cholesterol value. Although the advanced form of AMD was found in $13 \%$ of the cooperating AD patients $(n=68)$, the early form was seen only in $14 \%$. There was no significant difference in the occurrence of AMD in the AD subgroups showing different apoE isoforms.

Conclusions: A higher frequency of apoE 2 in AMD patients, and a higher frequency of apoE 4 in Alzheimer's have been found in the South-Western region of Hungary. The frequency of early AMD in AD patients was lower than expected from the population-based studies. The analysis whether CFH polymorphism influences the AMD-occurrence in AD patients is underway.

Key words: Apolipoprotein E, age-related macular degeneration and Alzheimer's disease.

$11: 59$

Lecture 15. K. Kránitz, J. Németh, Z. Z. Nagy (Budapest): Characteristics of corneal reshapening in progressive keratoconus after cross-linking therapy assessed by Scheimpflug-camera

Purpose: To evaluate geometrical shape factors of corneas before and after corneal cross-linking therapy (CXL) in progressive keratoconus.

Methods: Scheimpflug imaging of anterior segments was performed with Pentacam (Oculus, Wetzlar, Germany) in 15 eyes of 15 patients with progressive keratoconus before and after CXL.

The following corneal parameters have been evaluated before and 6 months after the treatment: anterior chamber depth (ACD), anterior chamber volume $(\mathrm{ACV})$, anterior chamber angle (ACA), back elevation (BE), asphericity of anterior corneal surface (Q-val), central and thinnest corneal thickness (CCT and ThCT) and average keratometry values (aveK). Statistical comparison was performed by T-test for dependent samples.

Uncorrected and best spectacle corrected visual acuity (UCVA and BSCVA) and manifest refraction spherical equivalent (MRSE) were also determined.

Results: Values of CCT and ThCT decreased statistically significantly 6 month after CXL. We found significant decrease in the values of BE $(p=0.043)$ and ACD $(p=0.006)$ after the surgery, however no such difference was found regarding ACV values $(p>0.05)$. Asphericity of the anterior corneal surface changed from -0.86 to $-0.70(\mathrm{p}=0.002)$. ACA increased significantly after CXL $(\mathrm{p}=0,047)$. AveK of keratoconic corneas decreased significantly after CXL from 48.04 D to 46.90 D ( $p<0.001$ ).

Significant improvement could be identified in UCVA (mean from 0.17 to 0.31 ; $\mathrm{p}=0.001$ ), but not in BSCVA (mean from 0.59 to $0.62 ; \mathrm{p}>0.05$ ) after CXL. MRSE decreased from $-5.06 \mathrm{D}$ to $-3.17 \mathrm{D}$ after 6 month $(\mathrm{p}=0.045)$.

Conclusions: After cross-linking therapy the shape of keratoconic corneas undergoes a kind of reshapening. This process is able to improve UCVA and decrease MRSE dioptres.

Key words: Cornea, keratoconus, cross-linking, Scheimpflug-camera.
Bemerkungen 
LIDER, TUMORE, GLAUKOM, DIV. Vorsitz: H. Reitsamer

1. P. Marvan, A. Redlberger, M. Emesz (Salzburg): Freies autologes Tarsustransplantat zur Rekonstruktion der hinteren Lamelle nach excisioneller Lidtumor-Chirurgie (Vortrag) - Autologous tarsal graft for posterior lamellar reconstruction following tumor surgery of the eyelid

Problemstellung: Große durchgreifende Liddefekte infolge exzisioneller Tumorchirurgie erfordern eine Rekonstruktion der vorderen und hinteren Lamelle des Lides. Diese Substanzdefekte können durch interponieren eines freien autologen Tarsustaransplantates kombiniert mit einem gestielten Hautlappen versorgt werden. Die Technik, wie an der Univ.-Augenklinik der PMU Salzburg eingesetzt, die postoperative Ergebnisse und die Komplikationen werden dargestellt.

Methode und/oder Patienten: In einer retrospektiven Studie wurden 10 Rekonstruktionen mit freiem Tarsustransplantat an der Univ. Augenklinik Salzburg analysiert. Es wurden Patienten mit großen Liddefekten nach Tumorexzision eingeschlossen. Der horizontal gemessene Substanzdefekt betrug in allen Fällen mindestens $>10 \mathrm{~mm}$. Es wurde Tarsusgewebe mit Bindehaut aus dem Oberlid des Partnerauges entnommen und in den Liddefekt eingenäht. Anschließend wurde die vordere Lamelle mit einem Hautverschiebelappen rekonstruiert. In Fällen von großen Substanzdefekten wurde das freie Tarsustransplantat mit gestielten Tarsustransplantaten kombiniert.

Ergebnisse: Wesentliche Wundheilungsstörungen oder Transplantatabstoßungen kamen nicht vor, die Hornhaut blieb unter dem Transplantat stets unversehrt. Im Besonderen ist anzumerken, dass weder eine postoperative Ektropiumtendenz, noch geringe Lidschlussdefizite ein Problem darstellten. Der durch die Tarsusentnahme entstandene Substanzdefekt am Oberlid des Spenderauges führte langfristig zu keinen klinischen Symptomen. Das kosmetische Ergebnis war in allen Fällen zufriedenstellend, kein Patient musste im Verlauf nachkorrigiert werden.

Schlussfolgerungen: Das freie autologe Tarsustransplantat des Partnerauges stellt eine gute Möglichkeit zu Rekonstruktion der hinteren Lidlamelle bei kleinen Substanzdefekten dar. Bei ausgedehnten Defekten im Unterlid hat es sich in Kombination mit gestielten Tarsustransplantaten bewährt. Zur Rekonstruktion großer Defekte der hinteren Lamelle des Oberlides eignet sich Schleimhaut vom harten Gaumen besser.

Schlüsselwörter: Freies autologes Tarsustransplantat, Lidtumore, Basaliom, Lidchirurgie.

Key words: Autologous tarsal graft, tumors of the lid, basal cell carcinoma, lid surgery.

2. M. Emesz, A. Redlberger, G. Oberascher (Salzburg): Exenteratio orbitae Chirurgische und rekonstruktive Strategien - Langzeitergebnisse (Vortrag) - Orbital exenteration - surgical and reconstructive strategies- long time results

Problemstellung: Bei Malignomen der Nasennebenhöhlen mit Beteiligung der Orbita und Infiltration der Periorbita war lange Zeit eine totale extensive Exenteration unumgänglich. Heute wird wird eine radikale Tumorexstirpation bei Erhaltung von möglichst viel Orbitagewebe angestrebt. Bei subtotaler extensiver 
Exenteration mit Erhalt von Bulbus und Weichteilgewebe der Orbita steht die Rekonstruktion der knöchernen Orbitawände im Vordergrund. Bei totaler und total extensiver Exenteration sollte ein primärer rekonstruktiver Weichteilaufbau der Orbita angestrebt werden.

Methode und/oder Patienten: An der Univ.-Klinik für Hals-Nasen-Ohrenheilkunde und an der Univ.-Augenklinik Salzburg wurden 16 Patienten mit Malignomen im Bereich der Orbita und Schädelbasis mit Orbitabeteiligung operiert. Bei 8 Patienten wurden im Rahmen einer subtotalen extensiven Exenteration knöcherne Orbitawände mittels Titanmesh ersetzt. Allenfalls fehlendes Weichteilgewebe wurde mittels Muskulus temporalis Lappens ersetzt. Bei 8 Patienten wurde aufgrund einer primären Orbitainfiltration oder einer Infiltration der Augenmuskeln bei von den Nasennebenhöhlen in die Orbita einbrechenden Malignomen eine totale oder eine total extensive Exenteration durchgeführt. Zur Weichteilrekonstruktion wurden hier Spalthaut oder ein mikrovaskulär anastomosierter Lappen herangezogen.

Ergebnisse: Die Patienten nach Orbitawandrekonstruktion mittels Titanmesh zeigten postoperativ alle ein gutes funktionelles und ästhetisches Ergebnis, eine freie Motilität, keine Doppelbilder und keinen Visusverlust. Patienten nach totaler extensiver Exenteration, die mittels mikrovaskulär anastomosiertem Lappen rasch primär rehabilitiert wurden, zeichneten sich durch ein zufriedenstellendes ästhetisches Ergebnis, Schmerzfreiheit sowie das Fehlen von sino - orbitalen Fisteln aus. Dies wirkte sich auch positiv auf die postoperative Radiotherapie aus.

Schlussfolgerungen: Auch bei großen von den Nasennebenhöhlen ausgehenden Karzinomen mit Orbitabeteiligung ist durch interdisziplinärem Vorgangsweise mittels radikaler Tumorexzision und begleitender Radiatio eine Heilung anzustreben. Dabei ist bei fehlender Tumorinfiltration der Augenmuskeln ein Bulbuserhalt möglich. Der Ersatz der knöchernen Orbitabegrenzungen mittels Titanmesh führt zu guten funktionellen und ästhetischen Ergebnissen. Fehlendes Weichteilgewebe kann durch Spalthaut, durch Temporalismuskellappen oder mikrovasulär gestielte Lappen ersetzt werden.

Schlüsselwörter: Orbitaexenteration, Periorbita, Titan mesh, Nasennebenhöhlentumore, Orbitarekonstruktion.

Key words: Exenteratio orbitae, periorbita, titan mesh, sinonasal tumors, orbital reconstruction.

3. A. Ettl (St. Pölten): Richtlinien zur Diagnostik und Therapie von Orbitatumoren (Kurzvortrag) - Guidelines for Diagnosis and Therapy of Orbital Tumors

Problemstellung: Präsentation der durch den Autor für die Kommission „Okuloplastische Chirurgie“ der ÖOG ausgearbeiteten Richtlinien betreffend die Diagnostik und Therapie von Orbitatumoren.

Methode und/oder Patienten: Übersichtsvortrag.

Ergebnisse: Nach Präsentation einer systematischen Klassifikation der wichtigsten Orbitatumore werden klinische, bildgebende und sonstige Untersuchungsmethoden zur Diagnostik orbitaler Raumforderungen mit dem Ziel eines standardisierten Vorgehens präsentiert. Danach werden die therapeutischen Möglichkeiten bei orbitalen Raumforderungen besprochen.

Schlussfolgerungen: Eine fundierte diagnostische Abklärung ist die Voraussetzung einer erfolgreichen Behandlung orbitaler Raumforderungen durch chirurgische Maßnahmen (Orbitotomie, Kraniotomie, Exenteratio), Bestrahlung (z.B. fraktionierte externe stereotaktische Radiatio), Chemotherapie oder andere medikamentöse Behandlungsoptionen.

Schlüsselwörter: Orbita, Tumor, orbitaler Tumor, Orbitotomie, Diagnose, Therapie, Richtlinien.

Key words: Orbit, tumor, orbital tumor, orbitotomy, diagnosis, therapy, guidelines.
Bemerkungen 
4. C. Fischer, M. Emesz, W. Riha, P. Marvan (Salzburg): Die intra- und paraläsionale Steroidinjektion als primäre Therapie des Chalazions (Kurzvortrag) - intralesional steroid-injection as first-line chalazion therapy

Problemstellung: Die chirurgische Excision (meist Inzision und Küretage) bei therapieresistenten Chalazien galt bisher als Standardtherapie. Sie wird von Patienten oft als durchaus belastend empfunden. Als minimal invasive Alternative dazu bietet sich die lokale Injektion von Steroiden an.

Methode und/oder Patienten: Seit Herbst 2009 wurden an der Universitätsaugenklinik Salzburg bei 80 Patienten Chalazien, die sich auf lokale antibiotische Therapie nicht oder nicht ausreichend zurückgebildet hatten, initial mittels intra- und paraläsionaler Cortison-Injektion therapiert. Dazu wurde nach klinischer Begutachtung und Vermessung der Läsionen, bei reizfreien, seit mindestens einem Monat bestehende Chalazien $5 \mathrm{mg}$ (in 0,1 ml) Triamcinolon (Volon-A) in das Lid injiziert. Die Nachuntersuchung erfolgte nach 2-8 Wochen, wobei einerseits der Lokalbefund klinisch beurteilt wurde und andererseits mittels detailliertem Fragebogen die Patientenzufriedenheit erhoben wurde. Dabei wurde auch nach den empfundenen Schmerzen während der Injektion, sowie nach der Zeitdauer bis zur subjektiv bemerkten Größenabnahme des Chalazions gefragt.

Ergebnisse: Es kam bei 70-80\% der Patienten zu einer zufriedenstellenden Größenabnahme bzw. zu einer vollständigen Remission des Chalazions, wobei die maximale Größenabnahme in der ersten Woche nach der Injektion beobachtet werden konnte. Nur in einigen Fällen war noch eine Küretage notwendig, Rezidive nach Injektionen zeigten sich nur in geringer Prozentzahl. 95\% der Patienten gaben an, mit dem Engriff sehr zufrieden oder zufrieden zu sein; die durch die Injektion verursachten Schmerzen wurden von fast allen Patienten als gering eingestuft und 95\% der Patienten würden den Eingriff wieder vornehmen lassen.

Schlussfolgerungen: Die Injektion von Steroiden ist eine wirksame und komplikationsarme Initialtherapie zur Behandlung von reizfreien Chalazien. Als einfache Alternative zu der - oft als schmerzhaft und unangenehm empfundenen operativen Küretage scheint diese Methode besonders geeignet zu sein. Im Falle eines Rezidivs nach zweimaliger Injektion sollte jedoch in allen Fällen eine chirurgische Excision mit histologischer Aufarbeitung des Materials zum Ausschluss eines malignen Geschehens vorgenommen werden.

Schlüsselwörter: Chalazion, Triamcinolon, intraläsionale Injektion, Lid.

Key words: Chalazion, triamcinolon, intralesional injection, lid.

5. A. Redlberger, P. Marvan, M. Rasp, M. Emesz (Salzburg): Laterale Orbitotomie und Refixation der lateralen Orbitawand unter Verwendung von selbstauflösenden Osteosynthesematerial - Diagnostische und therapeutische Aspekte anhand eines Fallberichts (Kurzvortrag) - Lateral orbitotomy and self-resolving osteosynthesis devices - Diagnostic and therapeutic aspects presented in a case report

Problemstellung: Das kavernöse Hämangiom ist der häufigste gutartige Orbitatumor im Erwachsenenalter. Es ist ein gut abgekapselter vaskulärer Tumor bestehend aus dilatierten, mit Blut gefüllten Gefäßhohlräumen, der sich überall in der Orbita entwickeln kann und am häufigsten im mittleren Alter auftritt. Es kann sich als Zufallsbefund oder mit Visusabfall und Proptosis manifestieren. Bei Größenzunahme ist eine chirurgische Exzision indiziert. Im dargestellten Fall erfolgte der Zugang zur Orbita über eine laterale Orbitotomie, wobei zur Refixation der latealen Orbitawand ein selbstauflösendes Osteosynthesematerial verwendet wurde.

Methode und/oder Patienten: Ein 33-jähriger Patient wurde im Juli 2009 mit einem tastbaren Tumor im Bereich der lateralen rechten Orbita an der Universitätsklinik für Augenheilkunde und Optometrie der PMU Salzburg vorstellig. Der Tumor führte bereits bei der Erstvorstellung zu einem Exophthalmus und Hö- 
herstand des Bulbus, die Abduktion war nur eingeschränkt möglich. Da es im Verlauf zu einer Progression des Exophthalmus kam, wurde die Indikation zur chirurgischen Exzision gestellt. Als operativer Zugang zur Orbita wurde eine laterale Orbitotomie über eine Kanthotomie gewählt. Nach Osteotomie konnte der Tumor problemlos in toto entfernt werden, wobei die anschließende Refixation der lateralen Orbitawand mit einem selbstauflösenden Osteosynthesematerial erfolgte.

Ergebnisse: Der histologische Befund ergab ein kavernöses Hämangiom. Postoperativ zeigte sich ein sehr zufriedenstellendes kosmetisches Ergebnis, es war kein Exophthalmus mehr nachweisbar und der Visus stieg um zwei Zeilen an. Es fand sich ein geringer Tieferstand des Bulbus rechts und der Patient hatte anfangs deutliche Doppelbilder, welche im Verlauf besser wurden und schließlich mit einer Prismenbrille korrigiert werden konnten. Das Osteosynthesematerial war sechs Monate nach der Operation im Seitenvergleich kaum tastbar.

Schlussfolgerungen: Das kavernöse Hämangiom zählt zu den wichtigsten Differentialdiagnosen von Raumforderungen in der Obrita beim Erwachsenen. Bei Progredienz und Symptomen ist eine chirurgische Entfernung in toto indiziert, die zumeist auch gut möglich ist, da der Tumor gut abgekapselt ist. Wird nach einer Orbitotomie zur Knochenrefixation ein selbstauflösendes Osteosynthesematerial verwendet, können Probleme mit fühlbaren Implantaten, Schraubenbewegungen, chronischen Reizzuständen und gegebenenfalls eine Folgeoperation zur Implantatentfernung vermieden werden.

Schlüsselwörter: Exophthalmus, Orbitatumor, Hämangiom, Orbitachirurgie, laterale Orbitotomie, Osteosynthese.

Key words: Exophthalmus, orbital tumor, haemangioma, orbital surgery, lateral orbitotomy, osteosynthesis.

6. E. Lindner, A. Barounig, B. Langner-Wegscheider, M. Weger, N. Ardjomand, Y. El-Shabrawi (Klagenfurt): Einfluss von IL2RA und IL7RA Genpolymorphismen auf HLA-B27 positive Uveitis und Uveitis intermedia (Kurzvortrag) - Influence of IL2RA and IL7RA gene polymorphisms on HLA-B27 positive uveitis and intermediate uveitis

Problemstellung: Ziel der Studie ist die Rolle von IL2RA und IL7RA Genpolymorphismen (rs2104286, rs12722489 and rs6897932) in HLA-B27 positiver Uveitis, in HLA-B27 negativer Uveitis und in Uveitis intermedia näher zu bestimmen.

Methode und/oder Patienten: 145 Patienten mit HLA-B27 positiver, akuter Uveitis anterior, 10 Patienten mit HLA-B27 negativer, akuter Uveitis anterior und 84 Patienten mit Uveitis intermedia, 132 HLA-B27 negative Kontrollen und 61 HLAB27 positive Kontrollen. Der Genotyp wurde mittels PCR bestimmt. Die Daten wurden mit Chi-Quadrat Test und Fisher's Exakt Test ausgewertet.

Ergebnisse: Eine signifikante Assoziation von rs2104286 mit Uveitis intermedia und HLA-B27 negativer Uveitis wurde festgestellt. HLA-B27 negative Uveitiden waren nicht mit rs2104286 assoziiert. Keine signifikanten Assoziationen wurden mit rs12722489 und rs6897932 gefunden.

Schlussfolgerungen: Da rs2104286 mit Uveitis intermedia, nicht aber mit HLAB27 negativer Uveitis assoziiert ist, kann auf Unterschiede in der Pathogenese dieser beiden Krankheiten geschlossen werden.

Schlüsselwörter: HLA-B27 positive Uveitis, Uveitis intermedia, IL2RA, IL7RA, Genpolymorphismen.

Key words: HLA-B27 positive uveitis, intermediate uveitis, IL2RA, IL7RA, gene polymorphisms. 
7. P. Allegri, A. Mastromarino (Lavagna/Genua): Wirkung des oralen Kurkumin Phospholipid bei der Behandlung von chronischen vorderen Uveitisrelapsen (Kurzvortrag) - Efficacy of oral phospholipidic curcumin in the management of chronic anterior uveitis relapses

Problemstellung: Curcumin has been successfully applied to treat inflammatory conditions in experimental research and in clinical trials. The purpose of our study is to evaluate the efficacy of an adjunctive-to-traditional treatment with curcumin-phosphatidylcholine complex administered twice a day in recurrent anterior uveitis of different etiologies.

Methode und/oder Patienten: The study group consisted of 106 patients who completed a 18-month follow-up therapeutic period. We divided the patients into 3 main groups of different uveitis origin: group 1 (autoimmune uveitis), group 2 (herpetic uveitis), and group 3 (different etiologies uveitis).

Ergebnisse: First end point was the evaluation of relapses frequency in all treated patients, before and after curcumin-adjunctive treatment, followed by the number of relapses in the 3 etiological groups. Wilcoxon signed-rank test showed a P.

Schlussfolgerungen: Our study, in conclusion, is the first to report potential therapeutic role of curcumin and its efficacy in eye relapsing diseases, such as anterior uveitis, and points out other promising curcumin-related benefits in eye inflammatory and degenerative conditions, such as dry eye, maculopathy, glaucoma, and diabetic retinopathy.

Schlüisselwörter: Kurkumin, Uveitis.

Key words: Curcumin, anterior recurrent uveitis, phosphatidylcholine-boundcurcumin (Meriva).

8. L. Jampol (Chicago): White-dot-Syndromes: Klinische Charakteristika und Assoziation mit Autoimmunerkrankungen (Vortrag) - White-dot-Syndromes: Clinical characteristics and association to autoimmune diseases

Problemstellung: Die White-dot-Syndrome umfassen eine große Gruppe idiopathischer Erkrankungen der Netzhaut und der Choroidea mit vermuteter immunologischer Genese. Dazu zählen MEDWS, MFC, PIC, APMPPE, AZOOR, serpiginöse Choroiditis und andere verwandte Erkrankungen, die zu Visusverlust führen, normalerweise keine systemische Manifestation aufweisen und vor allem Frauen betreffen. Bei keiner dieser Erkrankungen sind der Auslöser und die pathogenetische Entwicklung bisher sicher bekannt. Die aktuelle Hypothese ist, dass die White-dot-Syndrome zu den Autoimmunerkrankungen zählen, die durch die individuelle genetische Prädispostion und Umweltbedingungen verstärkt werden.

Methode und/oder Patienten: Die Prävalenz von Autoimmunerkrankungen wurde in Patienten mit White-dot-Syndromen und deren Verwandten ersten und zweiten Grades untersucht. Die Krankheitsbilder wurden ophthalmologisch exakt phänotypisiert. Funktionelle Parameter wurden bestimmt, der intraokulare Befund komplett dokumentiert mit sämtlichen diagnostisch relevanten Maßnahmen wie Ophthalmoskopie und Fundusphotographie, Fluoreszein- und Indozyaninangiographie, Fundusautofluoreszenz, optischer Kohärenztomographie, elektrophysiologischer Untersuchung. Es wurde ein ausführlicher serologischer/immunologischer Status erhoben.

Ergebnisse: Bei Patienten mit White-dot-Syndromen war die Häufigkeit von Autoimmunerkrankungen signifikant erhöht im Vergleich zur Kontrollgruppe. Diese Unterschiede zeigten sich auch in Verwandten ersten und zweiten Grades der Patienten mit White-dot-Syndromen. Auch wenn die zur Gruppe der White-DotSyndrome gerechneten Erkrankungen phänomenologische Gemeinsamkeiten aufweisen, ist bisher unklar, ob dies auch für die Ätiologie und die Pathogenese gilt. Bei keiner dieser Erkrankungen sind der Auslöser und die darauf aufbauende pathogenetische Entwicklung sicher nachgewiesen. Immunologische Reakti- 
onen auf vorangegangene virale Infekte verbunden mit einer genetischen Disposition scheinen aber ein gemeinsamer Nenner zu sein. Mit der vorliegenden Studie wird erstmals ein Zusammenhang mit Autoimmunerkrankungen nachgewiesen.

Schlussfolgerungen: White-dot-Syndrome sind eine relevante Gruppe von retinalen Erkrankungen. Eine exakte klinische Differenzierung ist wesentlich für die Diagnosestellung und zur Beurteilung der Prognose. Pathogenetisch handelt es sich offensichtlich bei der zugrundeliegenden Ursache um einen autoimmunologischen Prozess. Dies wird wesentliche Implikationen für das weitere therapeutische Prozedere haben.

Schlüsselwörter: White-dot-Syndrom, Autoimmunologie, Retina.

Key words: White-dot-syndrome, autoimmunology, retina.

9. M. Werner-Stöllinger, N. Lang, C. Wintersteller, M. Hohensinn, H. Reitsamer, W. Hitzl, G. Grabner (Salzburg): Sekundärglaukom bei Kindern mit Uveitis anterior: Ergebnisse der drucksenkenden Operation (Vortrag) - Surgical treatment for secondary glaucoma in children with anterior uveitis

Problemstellung: Das Sekundärglaukom stellt insbesondere bei Kindern eine schwere Komplikation einer Uveitiserkrankung dar. Unzureichender Erfolg der medikamentösen Drucksenkung erfordert oftmals chirurgische Methoden. In der Literatur werden den Drainageimplantaten im Vergleich zur Trabekulektomie oftmals bessere Langzeitergebnisse zugeschrieben.

Methode und/oder Patienten: Wir berichten von vier Kindern mit der Erstdiagnose einer Uveitis anterior im Alter von 2-11 Jahren. Das Sekundärglaukom war assoziiert mit idiopathischer Uveitis anterior und juveniler idiopathischer Uveitis (JIA). Die durchschnittliche Zeit zwischen Uveitisdiagnose und chirurgischem Eingriff war 9,8 Jahre. Der Zeitraum zwischen erstmals erhöhtem Augendruck und der Operation 6,1 Jahre. Alle Patienten waren immunsupprimiert und die Uveitis für mindestens zwei Monate vor dem Eingriff kontrolliert. Die Kinder benötigten präoperativ durchschnittlich 4,5 Antiglaukomatosa. Bei drei Pat wurde eine Trabekulektomie mit Mitomycin, bei einem eine Implantation eines Drainage-Systems (Ahmed-Valve), jeweils unter einer perioperative Steroidgabe in einer mittleren Dosierung von 18,0 mg täglich, durchgeführt.

Ergebnisse: In einer Nachbeobachtungszeit von 51 Monaten zeigen sich gute Ergebnisse. Der mittlere präoperative IOD von $26,3 \mathrm{~mm} \mathrm{Hg}$ konnte auf postoperativ 9,6 mmHg (ohne zusätzliche Glaukommedikation) gesenkt werden. Bei allen Patienten zeigt sich ein stabiler Visusverlauf ohne Progression des Glaukoms oder einer Katarakt. Zu einem Uveitisrezidiv nach der drucksenkenden Chirurgie kam es nach durchschnittlich 11,3 Monaten.

Schlussfolgerungen: Aus unseren eigenen Erfahrungen mit Sekundärglaukom bei Kindern mit Uveitis können wir sehr gute Ergebnisse betreffend der Druckund Entzündungskontrolle sowie ein geringes operatives Komplikationsrisiko berichten. Nach unserem Dafürhalten ist eine ausreichend potente immunsuppressive Therapie entscheidender für den Erfolg (d.h. eine langfristige Drucksenkung) als die gewählte Operationsmethode (Trabekulektomie mit MMC versus Glaukom-Drainagesysteme).

Schlüsselwörter: Uveitis, Kinder, Sekundärglaukom, Glaukomoperation, Immunsuppressive Therapie.

Key words: Uveitis, children, secundary glaucoma, surgical treatment, immunosuppressive therapy.

10. E. Stangler-Zuschrott (Wien): Reale oder larvierte Doppelbilder bei einseitiger hochgradiger Visusminderung. (Vortrag) - Real or obscure diplopia in cases of severe unilateral visual deterioration. 
Problemstellung: Das Vorkommen binokularer Diplopie bei einseitigem Ausfall des zentralen Sehens wird in der Begutachtung nicht immer anerkannt. Jedoch ist zu bedenken, dass etwa bei Augenmuskelparesen voll sehender Augen das Doppelbild von der Netzhautperipherie des gelähmten Auges generiert wird, normale Netzhautkorrespondenz vorausgesetzt.

Methode und/oder Patienten: Krankengut: 19 Fälle werden vorgestellt, Durchschnittsalter 68,4 Jahre. Visus führendes Auge 1,0-0,5, Begleitauge 0,1 und schlechter. Schielstellung convergent oder divergent, erst in höherem Alter aufgetreten. Grunderkrankungen: Maculopathie, Schielamblyopie, Zustand nach Ablatio retinae, Opticusatrophie. Nicht immer wird Doppeltsehen angegeben. Begriff der larvierten Diplopie: Die Binokularstörung äußert sich oft als Zukneifen eines Auges oder als subjektiv gesehener "Grauschleier“ trotz bester optischer Korrektur. Untersuchungmethoden: Orthoptische Tests, die auf bifovealem Angebot basieren, greifen nicht. Nur periphere Konturen werden doppelt gesehen. Die Schielwinkelmessung erfolgt mit dem Krimsky-Test, die Prüfung des peripheren Binokularsehens mit den Streifengläsern nach Bagolini. Letztere dienen auch zur Anpassung von Prismen.

Ergebnisse: 15 Patienten konnten mit Prismen befriedigend eingestellt werden. Bei Fortschreiten der Grunderkrankung war der Erfolg nur befristet. Die Prismen werden als Folie vor dem erkrankten Auge angebracht. Änderungen des Schielwinkels erfordern neuerliche Prismenanpassung. Bei 4 Fällen bestand keine ausreichende periphere Fusion bzw. war diese infolge langer Krankheitsdauer verloren gegangen.

Schlussfolgerungen: Binokulare Diplopie ist bei einseitigem Verlust der zentralen Sehschärfe möglich, wenn die Netzhautperipherie intakt ist und eine Schielstellung mit normaler Netzhautkorrespondenz vorliegt. Die Diagnose einer Binokularstörung wird durch indirekte Symptomatik erschwert, häufiges Zukneifen eines Auges dient als wichtiger Hinweis. Der Prismenausgleich des Schielwinkels ist in vielen Fällen möglich, bewirkt eine subjektive Verbesserung und wird dankbar angenommen.

Schlüsselwörter: Diplopie bei Senioren, larvierte Diplopie, einseitiger Visusverlust, Schielen im Presbyopenalter, Prismenkorrektur.

Key words: Age-related diplopia, obscure diplopia, unilateral visual loss, squint in presbyopic age, prismatic correction.

11. G. Kieselbach, M. Aigner, J. Troger, M. Kralinger (Innsbruck): Das Aufklärungsgespräch, eine Evaluierung von 57 Patienten vor und nach geplantem ophthalmologischen Eingriff (Vortrag) - Evaluation of informed consent in 57 cases before and after surgery

Problemstellung: Neben der forensischen Problematik ist die informierte Zustimmung zu einer Operation eine ethische Grundvoraussetzung. Die Einwilligung zu einer OP hängt von der Information und dem Verständnis des Patienten ab. Inwieweit der praktisch mögliche Zeitrahmen, die Indikation und das Umfeld dies beeinflussen, soll anhand dieser Befragung aufgezeigt werden.

Methode und/oder Patienten: 57 geplante Patienten wurden am Aufnahmetag vor und innerhalb 36h nach OP befragt. 50\% waren tagesklinisch, $75 \%$ der Patienten war über 70, 60\% der Fälle erschienen zu einer Kataraktoperation, $12 \%$ der Patienten wurden zu einer GlaukomOp und 28\% zu einer Vitrektomie aufgenommen.

Ergebnisse: Trotz unterschriebener Einverständnis gaben 15\% der Patienten vor und $18 \%$ nach der OP an nicht aufgeklärt worden zu sein, $40 \%$ wussten nicht, dass Komplikationen auftreten können und von den anderen $60 \%$ konnte nur jeder fünfte zumindest eine Komplikation nennen. Immerhin $12 \%$ aller Patienten wussten, dass eine Infektion auftreten könnte. 50\% wussten nicht, dass sie postoperativ Tropfen verwenden müssen. Jeder Dritte wusste postoperativ nicht, ob es Alternativen zur OP gegeben hätte, allerdings $98 \%$ gaben po an, sich wieder operieren zu lassen. Aufgrund der Datenfülle der Befragung, des Umfangs der 
Aufklärungsgespräche und der temporären Stresssituation ist eine eindeutige Schlussfolgerung unzulässig.

Schlussfolgerungen: Es muss diskutiert werden, ob die gängigen Aufklärungsgespräche zu geplanten ophthalmologischen OP in dieser umfangreichen Form die Patienten überfordern, die Möglichkeiten der Aufklärung nicht ausgenutzt werden, oder aber Zeit reduziert werden könnte, indem man die Patienten, wie mit den Beipackzetteln, alleine lässt, um im Anschluss daran, nur mehr konkrete Fragen beantwortet. Diese Befragung zeigt jedoch sehr einfach, dass der Wissenstand unserer Patienten auch nach einem Eingriff auf einem sehr geringem Niveau verbleibt, welches bei den seltenen Komplikationen naturgemäß große Probleme verursachen kann.

Schlüsselwörter: Aufklärungsgespräch.

Key words: Informed consent.

$14: 49$

12. C. Nischler, C. Wintersteller, P. Marvan, M. Emesz, G. Grabner, H. Reitsamer (Salzburg): Einfluss der Irispigmentierung auf den Augendruck und die Entstehung von Gesichtsfelddefekten und primären Offenwinkelglaukomen (Kurzvortrag) - The association of iris colour with intraocular pressure, visual field and primary open angle glaucoma in a large European cohort

Problemstellung: Ziel unserer Studie war es zu untersuchen, ob die Irispigmentierung einen Einfluss auf den Augendruck und die Entstehung von Gesichtsfelddefekten und primären Offenwinkelglaukomen (POWG) hat.

Methode und/oder Patienten: Insgesamt wurden für diese Studie an 5 europäischen Universitätskliniken 1848 Personen im Alter von 20 bis 90 Jahren inkludiert. Neben einer kompletten ophthalmologischen Untersuchung wurden Visus, Augendruck (Goldmann Applanationstonometrie), Gesichtsfeld und das Ausmaß der Linsentrübung mittels LOCS III (lens opacities classification system) evaluiert. Je nach Irisfarbe (hellblau, blau-grau, grün-braun, braun) wurden die Probanden in 4 Gruppen unterteilt. Die vier Gruppen unterschieden sich weder in Alter, Geschlecht, Visus, sphärischem Äquivalent oder Intensität der Linsentrübung.

Ergebnisse: Personen mit hellblau pigmentierter Iris hatten mit 14,71 $\mathrm{mmH}$ im Vergleich zu jenen mit blau-grauer (15,41 mmHg), grün-brauner (15,93 mmHg) und brauner (15,49 $\mathrm{mmHg}$ ) Irisfarbe einen signifikant niedrigeren Augendruck $(\mathrm{p}=0,01)$. Personen mit brauner Irisfarbe hatten deutlich mehr relative Gesichtsfelddefekte innerhalb der zentralen 20 und 50 und auch die Prävalenz von POWG's war mit 1,74\% deutlich höher bei Personen mit brauner Irisfarbe als bei jenen mit hell-blauer (1,32\%), blau-grauer $(1,40 \%)$ und grün-brauner $(1,10 \%)$.

Schlussfolgerungen: Unsere Studie zeigt, dass die Irisfarbe einen signifikanten Einfluss auf den Augendruck hat und möglicherweise bei Personen mit brauner Irisfarbe zum gehäuften Auftreten von Gesichtsfelddefekten und eines POWG's führt. Wodurch der unterschiedlich hohe Augendruck verursacht wird ist derzeit nicht geklärt. Möglicherweise führt die ständige Bewegung in einer dunkler pigmentierten Iris zu einer höheren Freisetzung von Pigment in das Kammerwasser und zur nachfolgenden Obstruktion des Kammerwasserabflusses.

Schlüisselwörter: Irispigmentierung, Augendruck, Gesichtsfelddefekte, primäres Offenwinkelglaukom.

Key words: Irispigmentation, intraocular pressure, primary open angle glaucoma.

13. H. Reitsamer, C. Wintersteller, S. Abri, M. Hohensinn, G. Grabner (Salzburg): Neue Mikroimplantate in der Glaukomchirurgie - erste Ergebnisse aus Salzburg (Vortrag) - New microshunts in glaucoma surgery - First results from Salzburg
Bemerkungen 
Problemstellung: Nach wie vor versuchen Glaukomchirurgen weltweit definierte Öffnungen in Augen operieren, durch die alternative Abflüsse für das Kammerwasser geschaffen werden sollen. Die bisher einzige dauerhafte und etablierte Methode für Augendrucksenkungen an das untere Ende des therapeutischen Bereichs ist die Trabekulektomie, sowie „Makroshunts“ (Ahmed, Baerveldt, Moteno) mit all ihren Vorteilen und Nachteilen. In dieser Arbeit werden die ersten Ergebnisse von 26 Patienten aus der Universitätsaugenklinik Salzburg, die mit neuartigen Miroshunts zur Augendrucksenkung ausgestattet wurden präsentiert.

Methode und/oder Patienten: Die Ergebnisse der Implantation des neuen Goldshunts $^{\odot}$, des AqueSys ${ }^{\odot}$ Shutns und des TransCend ${ }^{\odot}$ implantats bei Patienten mit primären Offenwinkelglaukom und Pseudoexfoliationsglaukom werden präsentiert.

Ergebnisse: Die Ergebnisse der Implantation des neuen Goldshunts ${ }^{\odot}$, des AqueSys ${ }^{\odot}$ Shunts und des TransCend ${ }^{\odot}$ implantats bei Patienten mit primären Offenwinkelglaukom und Pseudoexfoliationsglaukom werden präsentiert.

Ergebnisse: Es zeigten sich signifikante Unterschiede zwischen den Implantaten. Die Gruppe der Goldshunt Patienten zeigten in den ersten drei Monaten nach der Implantation sehr gute Senkungen des Augendrucks in den Zieldruckbereich. Nach etwas drei Monaten zeigte sich ein relativ rascher Druckanstieg, der zusätzliche Medikation oder einen erneuten Drucksenkenden chirurgischen Eingriff nötig machte. Die Implantate aus der Vorderkammer unter die Bindehaut (AqueSys) und aus der Vorderkammer in den suprachoroidalen Raum (TransCend) zeigen vielversprechende Ergebnisse.

Schlussfolgerungen: Die neuen Microimplantate zeigen sehr unterschiedliche Ergebnisse. Während Ergebnisse der Goldshuntimplantationen unter den Erwartungen geblieben sind, zeigen die neuen Tube Shunts TransCend und AqueSys deutlich vielversprechendere Resultate. Obwohl der Implantationsvorgang beider Implantate wesentlich einfacher ist als die Durchführung einer Trabekulektomie, zeigt der Zugang aus der Vorderkammer unter die Bindehaut doch ähnlich postoperative Komplikationen wie nach einer Trabekulektomie. Aufgrund der noch kurzen Nachbeobachtungszeit können derzeit keine Aussagen über die Langzeitdrucksenkung auf Basis der eigenen Daten erfolgen.

Schlüsselwörter: Mikroimplantate, Glaukom, Chirurgie, Aquesys, TransCend.

Key words: Microshunts, glaucoma, surgery, aquesys, TransCend.

14. C. Wintersteller, W. Hitzl, M. Hohensinn, G. Grabner, H. Reitsamer (Salzburg): Kontrollierte Cyclophotokoagulation (Vortrag) - Controlled cyclophotocoagulation

Problemstellung: Bei der transskleralen Cyklophotokoagulation zur Behandlung von Glaukomen bestand bisher das Problem in der Dosierung der Energie für den einzelnen Lasereffekt. Der Operateur hatte keine direkte Kontrolle über den Koagulationsvorgang. Das Zielgewebe ist nicht einsehbar und es kann daher aufgrund der hohen inter- und intraindividuellen Variation der optischen Gewebeparameter leicht zu Unter- oder Überdosierungen kommen. Unterdosierung ist therapeutisch wirkungslos, Überdosierung führt zum sogenannten Pop-Effekt, der schwere Schäden zur Folge haben kann.

Methode und/oder Patienten: Bei der Kontrollierten Cyclophotokoagulation (Coco) trifft ein Teil jener Laserstrahlung, welche die Koagulation bewirken soll, nach zahlreichen Streuprozessen sowohl in Sklera als auch im Ziliarkörper auf den Augenhintergrund, wird dort reflektiert, tritt durch die Pupille wieder nach außen und wird dort von einem Detektor registriert. Die zeitliche Änderung dieses Detektorsignals ist Maß für die Transmissionsänderung des Gewebes, während die Koagulation abläuft. Sowohl der Operateur als auch ein SteuerungsComputer können daher mit dieser Information den Koagulationsvorgang sehr gezielt abbrechen, sobald die erforderliche Energie in das Auge eingebracht ist.

Ergebnisse: Seit Juli 2010 wird die Kontrollierte Cyclophotokoagulation an der 
Universitätsaugenklinik in Salzburg angewendet. Daten von mehr als 40 Patienten werden erstmals vorgestellt, wobei die Drucksenkung - auch bei schwierigen Glaukomfällen - mit dem Effekt einer herkömmlichen transskleralen Cyklophotokoagulation vergleichbar ist. Über die ersten sechs Monate der Nachbeobachtungszeit wurden schwerwiegende Komplikationen bisher nicht beobachtet.

Schlussfolgerungen: Sicherheit und Präzision der transskleralen Cyklophotokoagulation werden durch die vorgestellte „Echtzeitsteuerung“ bei der Kontrollierten Cyclophotokoagulation wesentlich erhöht. Da das Risiko des Eingriffes wesentlich reduziert zu sein scheint, erlaubt dies eine breitere Anwendung des cyclodestruktiven Verfahrens.

Schlüsselwörter: Cyklophotokoagulation, Glaukomtherapie, Augeninnendruck. Key words: Cyclophotocoagulation, glaucoma therapy, intraocular pressure.

15. H. Reitsamer, C. Wintersteller, M. Hohensinn, M. Stöllinger, W. Hitzl, C. Nischler, G. Grabner (Salzburg): Überlegungen zur Progression von Gesichtsfeldparametern basierend auf den Ergebnissen der Salzburg Moorfields Collaborative Glaucoma Study (Vortrag) - Progression analysis in the Salzburg Moorfield Collaborative Glaucoma Study

Problemstellung: Die Feststellung ob eine Progression eines Glaukoms vorliegt oder nicht ist eine der schwierigsten Diagnosen bei der Beurteilung von Gesichtsfeldbefunden.

Methode und/oder Patienten: In einer longitudinalen prospektiven Kohorten Studie (Salzburg Moorfields Collaborative Glaucoma Study) wurden mittlere Jahresveränderungen der Mean Deviation und der Pattern Standard Deviation berechnet und klassifiziert. Die Steigungen der Regressionsgeraden wurden miteinander Verglichen und auf signifikante Unterschiede zwischen den Diagnosegruppen Normalbefund (kein Glaukom), Normalbefund mit großer Papillenexkavation und Primäres Offenwinkelglaukom untersucht. Alle Analysen wurden in SPSS mit einer Analysis of Variance und Post hoc Korrektur durchgeführt. Zur Feststellung der individuellen Abnahme der Sensitivität im Gesichtsfeld sind in Abhängigkeit der Durchführungsqualität im Mittel 6 Gesichtsfelduntersuchungen pro 2 Jahre vorzunehmen.

Ergebnisse: Es zeigten sich signifikante Unterschiede zwischen den Gruppen. Die Gruppe derer mit Normalbefund weist eine mittlere Zunahme der Mean Deviation um $0.41 \mathrm{~dB}$ (Normalgruppe), $0.5 \mathrm{~dB}$ (vergrößerte Papillen Exkavation) und 1.22 dB (Primäre Offenwinkelglaukome) pro 5 Jahresintervall ( $\mathrm{n}=1184$ ).

Schlussfolgerungen: Die vorliegenden Daten lassen eine neue Art der Behandlung zu, wie sie auch von den Guidelines der European Glaucoma Society empfohlen wird. Ins Zentrum der Aufmerksamkeit rückt nicht so sehr das Ereignis Progression, dessen Auftreten durch das Eintreten von willkürlich getroffenen Progressionslimits bestimmt wird. Vielmehr wird nun versucht, unter Berücksichtigung der Ausgangsschädigungen des Sehnerven (Papillenmorphometrie, Gesichtsfeldparameter, Sehnervenfaserschichtdicke, etc.), dem Alter und Gesundheitszustandes des Patienten sowie dem Verlust von Sensitivität/Sehnervenfasern pro Jahr eine Entscheidung für eine adäquate Therapie zu treffen.

Schlüsselwörter: Progression, Glaukom, Gesichtsfeld, populationsbasiert, Salzburg Moorfields Collaborative Glaucoma Study.

Key words: Progression, glaucoma, perimetry, population based, Salzburg Moorfields Collaborative Glaucoma Study.

16. W. Hitzl (Salzburg): 10-Jahres Inzidenz des Pseudoexfoliationssyndroms und des Pseudoexfoliationsglaukoms in Österreich (Vortrag) - 10-year incidence rates of pseudoexfoliation syndrome and pseudoexfolation glaucoma in Austria

Problemstellung: Untersuchung der 10-Jahresinzidenz von Pseudo-exfoliati- 
onssyndrom (PEX) und Pseudoexfoliationsglaukom (PXG) in einer Populationsbasierten Studie bei über 40 jährigen im Bundesland Salzburg. Weiters der Zusammenhang zwischen Alter und der 10-Jahresinzindenz von PEX und PXG.

Methode und/oder Patienten: Die Salzburg-Moorfields-Collaborative-Glaucoma-Study (SMCGS) ist eine prospektive Kohortenstudie im Rahmen eines Glaukom-Vorsorgeprogramms des Bundeslandes Salzburg. Die über Zeitungsinserate, Radioinformationen, Vorträge und Webpages adressierten Teilnehmer des Vorsorgeprogramms wurden einer augenärztlichen Untersuchung mit Spaltlampen, Biomikroskopie des vorderen und hinteren Augenabschnitts, einer quantitativen Papillenmorphometrie, Gesichtsfelduntersuchungen, Goldman Tonometrie bei der Erstuntersuchung, den Zwischenuntersuchungen bis hin zur 10-Jahresuntersuchung unterzogen.

Ergebnisse: Die Salzburg-Moorfields-Collaborative-Glaucoma-Study (SMCGS) ist eine prospektive Kohortenstudie im Rahmen eines Glaukom-Vorsorgeprogramms des Bundeslandes Salzburg. Die über Zeitungsinserate, Radioinformationen, Vorträge und Webpages adressierten Teilnehmer des Vorsorgeprogramms wurden einer augenärztlichen Untersuchung mit Spaltlampen, Biomikroskopie des vorderen und hinteren Augenabschnitts, einer quantitativen Papillenmorphometrie, Gesichtsfelduntersuchungen, Goldman Tonometrie bei der Erstuntersuchung, den Zwischenuntersuchungen bis hin zur 10-Jahresuntersuchung unterzogen.

Schlussfolgerungen: Die vorliegenden Daten erlauben die ersten Aussagen über die Inzidenzen von PEX und PXG in einer Stichprobe einer österreichischen Bevölkerung und ermöglichen darüber hinaus neue Rückschlüsse zum besseren Verständnis dieses Krankheitsbildes im internationalen Vergleich. Die Resultate zeigen eindeutig das erhöhte Risiko (10-fach) an einem PXG bei Vorliegen von PEX zu erkranken.

Schlüsselwörter: PEX, PEX-Glaukom, 10-Jahresinzidenz.

Key words: PEX, PEX glaucoma, 10-year incidence.

17. A. Boltz, D. Schmidl, M. Lasta, S. Kaya, G. Fuchsjäger-Mayrl, L. Schmetterer (Wien): Blutflussregulation des Sehnervenkopfes bei Änderungen des Augeninnendrucks und des arteriellen Blutdrucks (Vortrag) - Regulation of optic nerve head blood flow during combined changes in intraocular pressure and arterial blood pressure

Problemstellung: Autoregulation ist die Fähigkeit eines Gefäßbettes den Gefäßwiderstand sowohl an einen gesteigerten als auch an einen verminderten Perfusionsdruck anzupassen. Zahlreiche Studien zeigten, dass der retinale Blutfluss über weitgehende Veränderungen des Perfusionsdrucks autoreguliert wird. Leider liegen nur wenige Daten über die Blutflussautoregulation des Sehnervenkopfes vor. Dies wäre aber von besonderer Bedeutung, da viele wichtige Augenerkrankungen wie z.B. Glaukom mit einer veränderten Autoregulation einhergehen. Ziel dieser Studie war es, die autoregulatorischen Eigenschaften des Sehnervenkopfes zu untersuchen.

Methode und/oder Patienten: Zwei Studientage wurden mit 40 gesunden jungen Probanden durchgeführt. Der Perfusionsdruck wurde aus dem mittleren arteriellen Blutdruck (MAP) und dem intraokulären Druck (IOP) berechnet. Am ersten Studientag wurde separat der Effekt eines IOP- und eines MAP Anstiegs untersucht. Am zweiten Studientag wurden die Auswirkungen eines kombinierten IOP- und MAP Anstiegs erforscht. Der IOP wurde schrittweise mittels eines kleinen Saugnapfes, der auf die Sklera aufgesetzt wurde, erhöht. Der MAP wurde durch isometrische Übungen (Hockestellung) gesteigert. Der Blutfluss des Sehnervenkopfes wurde mittels Laser Doppler Flowmetrie gemessen. Für die Korrelationsanalyse wurden die Werte aller Probanden nach IOP und MAP gepoolt.

Ergebnisse: Wurden die Daten nach den MAP Werten in Gruppen eingeteilt, zeigte sich eine hochsignifikante Korrelation zwischen dem Blutfluss des Sehnervenkopfes und IOP ( $\mathrm{p}<0.001)$. Die Korrelation zwischen dem Blutfluss und MAP war viel
Bemerkungen 
weniger ausgeprägt bei Gruppierung der Daten nach den IOP Werten $(\mathrm{p}<0.05)$.

Schlussfolgerungen: Unsere Daten bestätigen die bisher publizierten Beobachtungen, dass der Blutfluss des Sehnervenkopfes eine autoregulatorische Kapazität bei Schwankungen des Perfusionsdrucks zeigt. Darüberhinaus weisen unsere Daten darauf hin, dass der Blutfluss besser bei Veränderungen des MAP als bei einer Erhöhung des IOP autoreguliert wird.

Schlüsselwörter: Blutfluss, Autoregulation, Sehnervenkopf.

Key words: Blood flow, autoregulation, optic nerve head.

KAFFEPAUSE 15:39 - 16:30

\section{HORNHAUT UND REFRAKTIVE CHIRURGIE}

Vorsitz: J. Stoiber

16:30

18. B. Steger, L. Speicher, W. Philipp (Innsbruck): Keimspektrum und Risikofaktoren der schweren infektiösen Keratitis (Vortrag) - Etiology and therapy of severe infectious keratitis

Problemstellung: An der Universitätsklinik für Augenheilkunde und Optometrie der Medizinischen Universität Innsbruck wurde seit 2007 eine Zunahme der Zahl schwerer infektiöser Keratitiden verzeichnet. Das Ziel dieser Studie war es, mikrobielle Charakteristika und Antibiotikaresistenzen dieser Fälle zu beschreiben. Es wurde versucht, Risikofaktoren zu identifizieren und die Rationalität der empirischen Initialtherapie zu prüfen.

Methode und/oder Patienten: Es wurde eine retrospektive Analyse von 43 an der Augenklinik Innsbruck stationär behandelten Fälle infektiöser Keratitis von Jänner 2007 bis Juni 2010 durchgeführt. Die Einschlusskriterien umfassten eine Symptomdauer vor Aufnahme von unter 4 Wochen und die Diagnose einer infektiösen Keratitis aus einem positiven Keimnachweis und/oder klinischem Ansprechen auf entsprechende antimikrobielle Therapie. Ausschlusskriterien waren nosokomial erworbene Keratitiden, Zustand nach Augenoperation innerhalb der letzten 4 Wochen und Zustand nach stationär behandelter infektiöser Keratitis am gleichen Auge. Als negatives Outcome wurden die Notwendigkeit einer perforierenden Keratoplastik, eine Enukleation und ein bestkorrigierter Visus von unter 0,1 drei Monate nach der stationären Aufname definiert. Zur statistischen Analyse wurden der Fisher Exact Test eingesetzt.

Ergebnisse: $79,1 \%$ der Fälle waren bakterieller Genese, 11,6\% durch Pilze und 9,3\% durch Acanthamöben verursacht. Als Risikofaktoren wurden in 55,8\% das Tragen von Kontaktlinsen, in 37,2\% eine Hornhautoberflächenpathologie identifiziert. Die Kontaktlinsen assoziierter Keratitis (CLRK) war zu 66,7\% durch Pseudomonas, die Nicht - CLRK (NCLRK) zu 57,0\% durch Staphylokokken bedingt. Ein negatives Outcome war in 32,5\% der Fälle zu verzeichnen. Die Art der topischen Vorbehandlung vor stationärer Aufnahme war signifikant mit dem Outcome assoziiert: 20,8\% zuvor unbehandelter Fälle, 33,3\% der nur antibiotisch vorbehandelten Fälle, und 71,4\% der mit Antibiotikum und Steroid vorbehandelten Fälle hatten ein negatives Outcome. Die Beschwerdedauer vor stationärer Aufnahme hatte prognostische Konsequenzen: Eine Dauer von mehr als 3 Tagen ging mit einem höherem Risiko für ein negatives Outcome einher.

Schlussfolgerungen: Kontaktlinsen sind der wichtigste ätiologische Risikofaktor der schweren infektiösen Keratitis und stellen damit ein bedeutendes Gesundheitsrisiko dar. Eine Beschwerdedauer von mehr als drei Tagen von stationärer Aufnahme und eine Vorbehandlung mit Steroiden sind mit einem schlechteren Outcome assoziiert.

Schlüsselwörter: Kontaktlinsen assoziierte Keratitis, infektöse Keratitis.

Key words: Contact lens related keratitis, microbial keratitis.
Bemerkungen 
19. M. Dirisamer, J. Lie, I. Dapena, L. Ham, G. Melles (Linz): Transplantation Bemerkungen einer isolierten Bowman-Schicht bei persistierendem subepithelialem Haze nach Excimer-Laser-Oberflächenablation (Kurzvortrag) - Isolated Bowman layer transplantation to manage persistent subepithelial haze after excimer laser surface ablation

Problemstellung: Die prospektive Evaluierung persistierenden subepithelialen Haze nach Excimer-Laser-Oberflächenablation mit einer isolierten BowmanSchicht-Transplantation zu behandeln.

Methode und/oder Patienten: Bei einem 21-jährigen Patienten mit einem persistierenden, dichten subepithelialen Haze nach photorefraktiver Keratektomie, ohne Besserungstendenz nach einer zweiten Laserbehandlung, wurde ein Flap mit einem Durchmesser von 9,0 mm und einer Dicke von $60 \mu \mathrm{m}$ exzidiert und eine Spender-Bowman-Schicht mit 9,0 mm Durchmesser auf das Stromabett nahtlos aufgelegt.

Ergebnisse: Zwei Monate nach der Transplantation stieg der mit Skleralinse korrigierte Visus von präoperativ 20/40 (0,5) auf 20/18 (1,2). Mittels optischer Kohärenztomographie stellte sich die transplantierte Bowman-Schicht als feine weiße Linie auf dem Empfängerstroma dar. Es trat kein weiteres Rezidiv auf.

Schlussfolgerungen: Dieses neue Verfahren der Transplantation einer isolierten Bowman-Schicht könnte Einsatz in der Behandlung von Trübungen des vorderen Hornhautstromas wie bei Haze nach Excimer-Laser-Oberflächenablation oder bei komplizierten epithelialen Wundheilungsstörungen finden.

Schlüsselwörter: Bowman Schicht, lamelläre Hornhauttransplantation, chirurgische Technik, photorefraktive Keratektomie, phototherapeutische Keratektomie.

Key words: Bowman layer, lamellar keratoplasty, corneal transplantation, surgical technique, photorefractive keratectomy, phototherapeutic keratectomy.

20. R. Strauß, D. Diesenberger, K. Schilcher, C. Alge-Priglinger, A. Wedrich, S. Priglinger (Linz): Untersuchung elastischer Eigenschaften der Hornhaut mit dem Atomkraftmikroskop (AFM) (Kurzvortrag) - Characterisation of corneal elasticity by atomic force microscopy

Problemstellung: In den letzten Jahren gewann das Corneal crosslink immer mehr an Bedeutung zur Behandlung des progressiven Keratokonus. Die Atomkraftmikroskopie (AFM) findet aufgrund ihrer analytischen Eigenschaften zunehmend Anwendung in der biologischen und biophysischen Forschung. Ziel dieser Studie war daher die Characterisierung der Elastizität von unbehandelten Hornhäuten und solchen nach Corneal crosslink-Verfahren.

Methode und/oder Patienten: Bei fünf Schweinehornhäuten wurde ein Standard Corneal crosslink-Verfahren mit der Applikation von Riboflavin und Exposition von UVA-Licht für $\left(370 \mathrm{~nm}, 3 \mathrm{~mW} / \mathrm{cm}^{2}\right)$ für 30 Minuten durchgeführt. Fünf weitere Hornhäute blieben unbehandelt und dienten als Kontrollkollektiv. Ultradünnschnitte der Proben wurden geschnitten und der Young' Modulus wurde mittels Atomkraftmikroskopie im anterioren Stroma ermittelt.

Ergebnisse: Es gelang die Darstellung von cornealen Kollagenfasern mit dem AFM. Es zeigte sich ein statistisch signifikanter Unterschied der mittleren Elastizität: diese betrug bei unbehandelten Hornhäuten $221 \mathrm{kPa}( \pm 144$ SD) und bei behandelten $283 \mathrm{kPa}$ ( \pm 140 SD; Mann-Whitney-U-test, $\mathrm{p}=0,003$ ).

Schlussfolgerungen: Die Atomkraftmikroskopie bietet neue Möglichkeiten für die Charakterisierung von Hornhäuten nach cornealem Crosslinkverfahren. Weitere Studien sind für ein noch besseres Verständnis der Effekte dieser Behandlungsmethode auf die corneale Ultrastruktur notwendig.

Schlüsselwörter: Keratokonus, Corneal Crosslink, Atomkraftmikroskop.

Key words: Keratokonus, corneal crosslink, atomic force microscopy. 
21. G. Schmidinger (Wien): Änderungen der Pachymetrie während dem

Problemstellung: Die Pachymetrie stellt den wichtigsten Sicherheitsfaktor bei einer Corneal Cross Linking (CXL) Behandlung dar. Ziel der Untersuchung ist es die Veränderung der Pachymetrie im Rahmen einer CXL-Behandlung zu untersuchen.

Methode und/oder Patienten: In dieser prospektiven, klinischen Untersuchung wurden 20 Augen von 20 Patienten mit einem progressiven Keratokonus einer CXL-Behandlung unterzogen und randomisiert in zwei Gruppen eingeteilt. Alle Behandlungen wurden nach dem Standardverfahren mit einer abrasio corneae durchgeführt. In Gruppe 1 wurde während der gesamten Behandlung ein Lidspekulum verwendet. In der zweiten Gruppe waren die Augen wären der Sättigungsphase der ersten 30 Minuten geschlossen. Patienten, deren Hornhaut nach 30 Minuten eine Pachymetrie von unter $400 \mu \mathrm{m}$ aufwiesen, wurden mit 0,1\% hypotoner Riboflavinlösung für 10 Minuten behandelt, um die Hornhaut zu quellen. Die Pachymetriemessungen wurden preaoperativ, nach der abrasio corneae und alle weiteren 10 Minuten durchgeführt. Dazu wurde die Online-OCT-Pachymetrie des Amaris Excimer Lasers (Schwind) sowie eine Ultraschallpachymetrie (SP-2000, Tomey) verwendet.

Ergebnisse: Die mittlere preaoperative Pachymetrie betrug in Gruppe $1466 \pm$ $23 \mu \mathrm{m}$ und $477 \pm 28 \mu \mathrm{m}$ in Gruppe 2. Nach der Epithelentfernung fand sich eine Restdicke der Hornhaut von $415 \pm 20 \mu \mathrm{m}$ in Gruppe 1 und $430 \pm 35 \mu \mathrm{m}$ in Gruppe 2. In Gruppe 2 mit geschlossenem Lid waren die Pachymetriemessungen während der ersten 30 Minuten weitgehend konstant, am Ende der 30 Minuten betrug die mittlere Hornhautdicke $428 \pm 50 \mu \mathrm{m}$. In der Gruppe 1 hingegen kam es zu einer signifikanten Abnahme der Hornhautdicke auf $355 \pm 23 \mu \mathrm{m}$ nach $30 \mathrm{Mi}$ nuten. Die Quellung mit hypotoner Riboflavinlösung konnte im Mittel eine Zunahme der Hornhautdicke von $54 \mu \mathrm{m}$ bewirken. Während der Bestrahlungsphase kam es in beiden Gruppen zu einer Abnahme der Pachymetrie. Der Effekt der Quellung in Gruppe 1 war nach 10 Minuten bereits wieder verloren.

Schlussfolgerungen: Die isotone Riboflavinlösung in 20\% Dextran führt bei geöffnetem Lid zu einer signifikant stärkeren Dehydrierung der Hornhaut als bei geschlossenem Lid. Die Verwendung einer hypotonen Riboflavinlösung ermöglicht eine kurzzeitige Rehydrierung der Hornhaut, dieser Effekt ist aber nach spätestens 10 Minuten wieder verloren. Da eine ausreichende Pachymetrie für eine sichere Behandlung unbedingt notwendig ist, sollte eine Behandlung in dünnen Hornhäuten nur unter größter Vorsicht durchgeführt werden.

Schlüsselwörter: Corneal Cross Linking, Keratokonus, Pachymetrie.

Key words: Corneal cross linking, keratoconus, pachymetry.

22. O. Seyeddain, J. Stoiber, J. Ruckhofer, G. Grabner (Salzburg): „Corneal Cross Linking“ (CXL) bei progredientem Keratoconus - 4-Jahres Ergebnisse (Vortrag) - „Corneal Cross Linking“ (CXL) treatment of progressive keratoconus - 4-years follow up

Problemstellung: Ziel dieser Langzeitstudie war der Nachweis eines langfristig anhaltenden Effekts der Riboflavin- und UVA-Licht induzierten Kollagenvernetzung (Corneal Cross Linking, CXL) bei progressivem Keratoconus.

Methode und/oder Patienten: Patienten mit einem objektivierbar nachgewiesenem progressiven Keratoconus (d.h. Änderung von Kmax um $\geq 1$ D, Verschlechterung des Visus im letzten Jahr) und einer Mindesthornhautdicke von $400 \mu \mathrm{m}$ werden seit Juni 2005 an der Univ.-Augenklinik Salzburg einem CXL nach dem Dresdner Protokoll unterzogen. In dieser retrospektiven Studie wurden Patienten mit maximalen Keratometriewerten von unter 60 Dioptrien (präoperativ) 
und einer mindestens 4 Jahre langen Nachbeobachtungszeit (maximal über 5 Jahre) eingeschlossen. Es wurden die höchsten Keratometriewerte mittels Hornhauttopographie (Keratron Scout), sowie die Endothel-Zellzahl mittels Spiegelmikroskop (Konan noncon Robo-CA) präoperativ, 1 Jahr und 3/4 Jahre postoperativ verglichen.

Ergebnisse: Von 21 monolateral operierten Patienten konnten 12 Patienten zur 4 Jahreskontrolle einberufen werden. Die maximalen Keratometriewerte verringerten sich signifikant im Mittel von präoperativ 48,92 $\pm 3,08 \mathrm{D}$ auf 48,36 $\pm 3,37 \mathrm{D}$ nach einem Jahr und 47,75 $\pm 3,13$ D nach mindestens vierjähriger Nachbeobachtungszeit. Die Endothel-Zellzahl blieb über den gesamten Kontrollzeitraum stabil (präoperativ: 2617 Zellen/mm² $\pm 414,1 \mathrm{Jahr}$ postoperativ 2569 Zellen/mm² \pm 289; 4 Jahre postoperativ 2676 Zellen $/ \mathrm{mm}^{2} \pm 302$ ). Kein Patient zeigte Komplikationen im Kontrollzeitraum.

Schlussfolgerungen: Obwohl die Anzahl der Patienten mit einer Nachkontrollzeit von länger als 4 Jahren gering ist und dadurch eine statistische Aussage nur begrenzt zu ziehen ist, deuten die Ergebnisse dieser ersten österreichischen Langzeitstudie auf eine langfristige Befundstabilisierung des Keratoconus nach CXL hin.

Schlüsselwörter: Corneal Cross Linking, Keratoconus, Cornea.

Key words: Corneal cross linking, keratoconus, cornea.

23. J. Stoiber, J. Ruckhofer, O. Seyeddain, G. Grabner (Salzburg): Deep Anterior Lamellar Keratoplasty (DALK) bei fortgeschrittenem Keratokonus (Vortrag) - Deep anterior lamellar keratoplasty (DALK) in patients with advanced keratokonus

Problemstellung: Die lamelläre anteriore Keratoplastik hat gegenüber der konventionellen perforierenden Keratoplastik den Vorteil, dass das eigene Endothel erhalten bleibt und so endotheliale Abstossungsreaktionen vermieden werden können. Allerdings kommt es oftmals zu Narbenbildungen im Interface zwischen Empfänger- und Spenderstroma. In den letzten zehn Jahren erlebte die lamelläre Keratoplastik eine Renaissance, vor allem in Form der tiefen lamellären Keratoplastik (deep anterior lamellar keratoplastiy, DALK), bei der im Idealfall die Präparation bis auf die Tiefe der Descemet'schen Membran (DM) durchgeführt wird und so Narbenbildungen im Interface vermieden werden können.

Methode und/oder Patienten: Im Zeitraum zwischen Juli 2008 und Dezember 2010 wurde an 28 Augen eine DALK durchgeführt. In allen Fällen lag ein fortgeschrittener Keratokonus vor. In allen Fällen wurde die Separierung der DM mittels „Big-Bubble“-Technik nach Anwar versucht. Eine partielle Trepanation auf 80\% der Hornhautdicke erfolgte mittels geführtem Trepansystem (GTS) bzw. Femtosekundenlaser. Die Injektion von Luft in das tiefe Stroma führt im Idealfall zu einer blasenförmigen Abhebung der DM („Big Bubble“). Anschließend wurde das Reststroma exzidiert und die DM freigelegt. Die Spenderhornhaut wurde - nach vorherigem Abziehen von DM und Endothel - mittels doppeltfortlaufender Naht fixiert. Postoperativ wurde zu den Zeitpunkten 3, 6 und 12 Monate nach Transplantation bzw. nach kompletter Nahtentfernung Refraktion, Visus, Astigmatismus sowie die Endothelzellzahl bestimmt.

Ergebnisse: Bei 16 von 28 Patienten (57\%) war eine intraoperative Abhebung der DM mittels „Big Bubble“ möglich. Bei 12 Patienten gelang die Abhebung nicht und es wurde eine manuelle Präparation bis auf Descemetniveau durchgeführt. In fünf Fällen (18\%) machte eine Perforation der DM bei der Präparation die Umwandlung in eine perforierende Keratoplastik erforderlich. Die visuelle Rehabilitation der Patienten nach DALK war vergleichsweise rasch, bei der 6 MonatsKontrolle wurde ein mittlerer bestkorrigierter Visus von 0,7 erreicht. Es wurden keine visusmindernden Vernarbungen im Interface beobachtet. Der mittlere bestkorrigierte Visus 3 Monate nach kompletter Nahtentfernung war 0,85 $( \pm 0,1)$, der mittlere Astigmatismus war 3,2 D. Die mittlere Endothelzelldichte zu diesem Zeitpunkt betrug $2267 \mathrm{Z} / \mathrm{mm}^{2}$. 
Schlussfolgerungen: Da die Vorderkammer in Rahmen der DALK nicht eröffnet wird, besteht im Vergleich zur konventionellen perforierenden Keratoplastik ein signifikant reduziertes Risiko für intraoperative Blutungen, Wundleckage, Endophthalmitis oder Sekundärglaukom. Ein Nachteil der Methode ist die vergleichsweise längere Operationszeit, vor allem zu Beginn der „learning curve“. Da das Endothel des Empfängers zur Gänze erhalten bleibt, sind endotheliale Abstoßungsreaktionen ausgeschlossen. Bei Durchführung einer „maximal tiefen“ lamellären Keratoplastik können zudem auch Visusergebnisse erreicht werden, die mit jenen nach perforierender Keratoplastik vergleichbar sind.

Schlüsselwörter: DALK, Hornhauttransplantation, Keratoplastik, Keratokonus. Key words: DALK, corneal transplantation, keratoplasty, keratoconus.

$17: 16$

24. M. Dirisamer, I. Dapena, L. Ham, G. Melles (Linz): Visus Ergebnisse der ersten 200 Descemet Membran Endothel Keratoplastiken (DMEK) (Kurzvortrag) - Visual results of the first 200 descemet membrane endothelial keratoplasty (DMEK) cases

Problemstellung: Evaluation der Visusergebnisse nach Descemet-Membran Endothelkeratoplastik (DMEK) zur Behandlung von Fuchs'scher Endotheldystrophie oder bullöser Endothelkeratopathie.

Methode und/oder Patienten: Bei 200 Patienten wurde eine DMEK zur Behandlung von Fuchs'scher Endotheldystrophie oder bullöser Endothelkeratopathie durchgeführt. Nach einer selbstschließende $3 \mathrm{~mm}$ Tunnelinzision wurde die Vorderkammer mit Luft gefüllt und die Descemet-Membran (DM) vom posterioren Stroma abgelöst. Anschließend wurde ein 9,5 mm DM-Transplantat durch die Tunnelinzision in die Vorderkammer injiziert. Das Transplantat wurde vorsichtig entfaltet und mittels Luftinsufflation gegen das Empfängerstroma gedrückt. Die Vorderkammer wurde für 45-60 min mit maximaler Luftfüllung belasen.

Ergebnisse: In 10 Augen (5\%) versagte das Transplantat bzw. war es nicht vollständig anliegend. Diese Patienten wurden erfolgreich mit einer sekundären „Descemet Stripping Endothelkeratoplastik“ (DSEK) versorgt. 22 Augen wurden aufgrund eines verminderten visuellen Potentials, 5 aufgrund einer „spontaneous clearence" von der Visus Analyse ausgeschlossen. Von den 163 DMEK-Augen erreichten im 3. postoperativen Monat 91\% (146/160) eine Sehschärfe von 0,5 oder besser und $65 \%(104 / 160)$ erreichten 0,8 oder besser. 6 Monate postoperativ erreichten 95\% (137/144) eine Sehschärfe von mindestens 0,5 oder besser.

Schlussfolgerungen: Die DMEK erlaubt eine schnelle und fast komplette visuelle Rehabilitation bei der Behandlung von endothelialen Hornhauterkrankungen.

Schlüsselwörter: Kornea, Descemet-Membran Endothelkeratoplastik (DMEK), posteriore lamelläre keratoplastik, Hornhauttransplantation, Descemet-Membran, Endothel, Visus, Fuchs, bullöse Keratopathie.

Key words: Endothelial keratoplasty, posterior lamellar keratoplasty, Descemet membrane, visual acuity, Fuchs, bullous keratopathy.

25. F. Prager, G. Schmidinger, M. Reiter, C. Skorpik, (Wien): Langzeitergebnisse nach Endokeratoplastik (EKP) (Vortrag) - Long-term results after endokeratoplasty

Problemstellung: Die hier verwendete Technik der Endokeratoplastik ist eine hintere lamelläre Keratoplastik bei der mittels eines Mikrokeratoms ein vorderer Hornhautlappen am Patientenauge geschnitten $(250 \mu \mathrm{m})$ und anschließend nach nasal umgeschlagen wird. Nach Trepanation erfolgt der Austausch der hinteren Lamelle mit der hinteren Spenderlamelle, welche in ähnlicher Weise vorher an der Vorderkammerbank präpariert wurde. Nach Readaption des vordere Lappen und Fixation mit Nähten, wird zur besseren Verbindung zwischen Spen-
Bemerkungen 
derlamelle und vorderen Hornhautlappen Luft in die Vorderkammer gefüllt, welche nach 2 bis 3 Minuten durch BSS Plus ersetzt wird. Die Nähte werden 4-6 Monate postoperativ entfernt.

Methode und/oder Patienten: Bei 53 Augen von 45 Patienten mit endothelial bedingten Hornhauttrübungen bei Fuchs'scher Endotheldystrophie, pseudophaker Keratopathie und bullöser Keratopathie nach Glaukom und nach Trauma, wurde zwischen Juni 2002 und September 2008 eine Endokeratoplastik durchgeführt. Die postoperativen Folgeuntersuchungen beinhalteten eine komplette Augenuntersuchung mit Spaltlampenuntersuchung inklusive Applantionstonometrie und Funduskopie, eine Bestimmung der bestkorrigierten Sehschärfe, eine Ultraschall-Pachymetrie der Hornhaut (Pachymeter SP-2000, Tomey Corporation, Nagoya, Japan) sowie eine Messung der Endothelzellzahl (Noncon ROBO-CA, Konan-Keeler, Kobe, Japan).

Ergebnisse: Das Durchschnittsalter aller Patienten war 70 Jahre. Der durchschnittliche Nachbeobachtungszeitraum betrug für alle Patienten 32 Monate. Der durchschnittliche bestkorrigierte Visus verbesserte sich von 0,21 präoperativ zu 0,61 nach 60 Monaten Nachbeobachtungszeitraum $(\mathrm{n}=10,+23$ Buchstaben, $p=0,009$ ). Die durchschnittliche Endothelzellzahl verringerte sich von 2797 Zellen $/ \mathrm{mm}^{2}$ präoperativ (Spenderhornhaut) zu 882 Zellen $/ \mathrm{mm}^{2}(\mathrm{n}=10,-68 \%$, p.<0.001) nach 60 Monaten. Die postoperative durchschnittliche Hornhautdicke nahm von $530 \mu \mathrm{m}(\mathrm{n}=32) 3$ Monate nach EKP auf $586 \mu \mathrm{m}(\mathrm{n}=15,+11 \%, \mathrm{p}=0.003)$ nach 60 Monaten zu. Durchschnittliches sphärisches Äquivalent und durchschnittlicher Astigmatismus nahmen leicht, aber nicht statistisch signifikant von +0,65 Dioprien (D) und +2,1 D ein Monat nach EKP auf +0,18 D und +1,75 D nach 60 Monaten ab. In 5 Augen (9\%) kam es zu einer Transplantabstoßung.

Schlussfolgerungen: Die Endokeratoplastik erwies sich als aussichtsvolle Alternative zur perforierenden Keratoplastik bei Patienten mit endothelial bedingter Hornhauttrübung oder Trübungen des hinteren Hornhautstromas. Vorteile sind vor allem der vergleichsweise geringe postoperative Astigmatimus, sowie der schnellere Heilungsverlauf. Die durchschnittliche postoperative Endothelzellzahl verringerte sich zwar vor allem in den ersten zwei postoperativen Jahren, stabilisierte sich aber anschließend im weiteren Beobachtungszeitraum.

Schlüsselwörter: Endotheliale Keratoplastik, Langzeitergebnisse.

Key words: Corneal transplantation, long-term follow-up.

$17: 30$

26. J. Stoiber, J. Ruckhofer, O. Seyeddain, G. Grabner (Salzburg): Die BostonKeratoprothese - visuelle Rehabilitation bei schwersten Veränderungen der Augenoberfläche (Vortrag) - The Boston-Keratoprosthesis - visual rehabilitation in patients with severe ocular surface disease

Problemstellung: Die visuelle Rehabilitation bei schweren Erkrankungen der Augenoberfläche - beispielsweise nach Verätzungen oder Verbrennungen - gilt seit langer Zeit als eine der großen Herausforderungen auf dem Gebiete der Ophthalmochirurgie. Die Implantation einer Keratoprothese stellt bei beidseitigen schweren Hornhauterkrankungen bzw. nach mehrfach gescheiterter Keratoplastik mitunter die letzte Möglichkeit zur Wiederherstellung eines funktionell brauchbaren Sehvermögens dar. Die Entwicklung der Boston Keratoprothese wurde in den 60iger Jahren von Claes Dohlman begonnen, Methode und Implantat wurden seither stetig weiterentwickelt.

Methode und/oder Patienten: Im Zeitraum von Juli 2007 bis November 2010 wurde an der Universitätsaugenklinik Salzburg bei insgesamt 22 Patienten die Implantation einer Boston-Keratoprothese durchgeführt. Die häufigste Diagnose war Verätzung $(n=9)$, gefolgt von bullöser Keratopathie nach multiplem Transplantatversagen $(n=7)$. Postoperativ wurden alle Patienten dauerhaft mit einer therapeutische Kontaktlinse versorgt. Eine topische Infektionsprophylaxe mittels Vancomycin wurde in allen Fällen durchgeführt.
Bemerkungen 
Ergebnisse: Bei allen Patienten konnte die Keratoprothese ohne Komplikationen implantiert werden. Bei 17 von 22 Patienten konnte durch die Implantation ein signifikanter Visusanstieg erzielt werden. Bei 3 Patienten musste die Prothese aufgrund lytischer Hornhautprozesse explantiert und durch ein konventionelles Transplantat $(n=1)$ bzw. eine neuen Boston-Keratoprothese $(n=2)$ ersetzt werden. In 19 Fällen blieb die Keratoprothese mittelfristig in situ, bei einer Followup Zeit von bis zu 3,5 Jahren. Bei unverändertem hinteren Augenabschnitt konnten mitunter sehr gute Visusergebnisse (bis zu 0,9) erreicht werden.

Schlussfolgerungen: Die Boston Keratoprothese eröffnet eine weitere Möglichkeit zur visuellen Rehabilitation bei primär ungünstigen Ausgangsbedingungen. Änderungen des postoperativen Regimes und Verbesserungen im Design führten in den letzten Jahren zu einer Verbesserung der mittel- und langfristigen Ergebnisse. Die Indikationen für die Boston-Keratoprothese umfassen hochgradige Hornhautvaskularisation nach beidseitiger schwerer Verätzung und bullöse Keratopathie nach mehrfach fehlgeschlagener Keratoplastik, sie ist aber für sehr trockene Oberflächenverhältnisse weniger gut geeignet. In diesen Fällen ist die Osteo-Odoto-Keratoprothese indiziert.

Schlüsselwörter: Verätzung, Keratoprothese, Limbusinsuffizienz, Transplantatversagen.

Key words: Corneal burn, keratoprosthesis, limbal deficiency, corneal graft failure. 


\title{
Freitag, 3. Juni 2011
}

\author{
$06: 30$ \\ 2. ÖAC/ÖOG - Lauf \\ Europahaus
}

08:00 Uhr 2. WISSENSCHAFTLICHE SITZUNG

Europahaus, Saal Europa

REFRAKTIVE CHIRURGIE UND INTRAOKULARLINSEN

Vorsitz: O. Findl, J. Ruckhofer

8:00

27. A. Dexl, O. Seyeddain, W. Riha, M. Hohensinn, T. Rückl, V. Reischl, G. Grabner (Salzburg): Das KAMRA ${ }^{\mathrm{TM}}$ intrakorneale Inlay zur chirurgischen Korrektur der Presbyopie - 3 Jahres Ergebnisse (Vortrag) - The KAMRA ${ }^{\mathrm{TM}}$ intracorneal inlay for the surgical correction of presbyopia: 3 years followup

Problemstellung: Die chirurgische Korrektur der Presbyopie ist die letzte große, derzeit noch ungenügend gelöste Herausforderung der refraktiven Chirurgie. Das KAMRA ${ }^{\mathrm{TM}}$ intrakorneale Inlay (vormals AcuFocus ${ }^{\mathrm{TM}}$ Corneal Inlay 7000) ist ein $10 \mu \mathrm{m}$ dünnes, 3,8 $\mathrm{mm}$ im Durchmesser großes intrakorneales Implantat, mit einer zentralen optischen Apertur von $1.6 \mathrm{~mm}$, welches aus PVDF/Carbon besteht. Es wird zur refraktiv-chirurgischen Korrektur der Presbyopie, dem Effekt einer stenopäischen Lücke entsprechend, in die Hornhaut des nicht dominanten Auge nach Präparation eines Flaps mit dem Femtosekundenlaser, eingesetzt.

Methode und/oder Patienten: Im Rahmen der FDA-Zulassungstudie erfolgte an der Univ.-Augenklinik der PMU Salzburg die monokulare Implantation des KAMRA $^{\mathrm{TM}}$ intrakornealen Inlays in das nicht dominante Auge von 32 natürlich emmetropen und presbyopen Patienten. Refraktion, Unkorrigierter Nah-, Intermediär-, und Fernvisus, sowie bestkorrigierter Fernvisus, Gesichtsfeldüberprüfung, und Komplikationen wurden über einen mittleren Nachbeobachtungszeitraum von 36 Monaten erhoben.

Ergebnisse: 3 Jahre postoperativ stieg der unkorrigierte Nah- bzw. Intermediärvisus von präoperativ Jg 6 bzw. 0,5 auf Jg 1 bzw. 0,8. Nach 36 Monaten hatten 97\% der implantierten Augen einen unkorrigierten Nahvisus von Jg 3 oder besser, und $91 \%$ erreichten einen unkorrigierten Intermediärvisus von 0,6 oder besser. Der mittlere unkorrigierte Fernvisus betrug nach 36 Monaten 1,0, wobei 100\% der Patienten zumindestens 0,6 erreichten. Innerhalb des Nachbeobachtungszeitraumes musste kein Inlay explantiert werden, und es wurden keinerlei inflammatorische Reaktionen beobachtet.

Schlussfolgerungen: Diese 3-Jahres Resultate untermauern den Stellenwert des KAMRA ${ }^{\mathrm{TM}}$ intrakornealen Inlays zur refraktiv-chirurgischen Korrektur der Presbyopie bei emmetropen und presbyopen Patienten als sicheres, effektives und potentiell reversibles Verfahren.

Schlüsselwörter: KAMRA, Presbyopie.

Key words: KAMRA, presbyopia.

28. T. Rückl, A. Dexl, W. Riha, O. Seyeddain, M. Hohensinn, M. Rasp, G. Grabner (Salzburg): Sicherheit und Wirksamkeit der Pocket-Technik zur Implantation des KAMRA TM corneal inlays bei der chirurgischen Korrektur der Presbyopie - Ein-Jahres Ergebnisse (Kurzvortrag) - Safety and effectiveness of the KAMRA TM corneal inlay implanted in presbyopic patients-pocket technique-12 month follow up 
Problemstellung: Das KAMRA ${ }^{\mathrm{TM}}$ Corneal Inlay (KCI) ist ein ringförmiges intracorneales Implantat (Außendurchmesser 3,8, Innendurchmesser 1,6 mm, Dicke $5 \mathrm{~mm}$ ) aus dem inertem Material PVDF mit ultradünner Carbonbeschichtung. Die dadurch erreichte Verkleinerung der optischen Apertur erhöht die Tiefenschärfe des Auges und führt zu einer Verbesserung des Visus im Nah- und Intermediärbereich, ohne signifikante Kompromittierung des Fernvisus. Im Rahmen der FDA-Zulassungsstudie erfolgte eine prospektive klinische Prüfung zur Beurteilung von Sicherheit und Wirksamkeit der „Pocket-Technik“ bei Implantation des KCIs als chirurgische Presbyopie-korrektur.

Methode und/oder Patienten: Zwischen Februar und Oktober 2009 erfolgte an der Univ.-Augenklinik Salzburg die Implantation des KCI's in die Hornhaut des nicht dominanten Auges von 24 emmetropen, presbyopen Patienten durch einen Chirurgen (GG). Nach Präparation einer Tasche (Tiefe im Mittel: 232,5 $\mu \mathrm{m}$ ) mittels des FS-Lasers wurde das Implantat über der optischen Achse positioniert. Einschlusskriterien waren ein unkorrigierter Nahvisus zwischen 0,2 und 0,5, sowie ein Fernvisus von $\geq 1,0$ auf dem zu operierenden Auge. Mittels Optec 6500P Vision Tester wurden vor dem Eingriff sowie im Rahmen aller Nachkontrollen (1 Woche, sowie 1, 3, 6, 9, und 12 Monate postop.) der Fern-, Intermediär-, und Nahvisus (sowohl unkorrigiert als auch bestkorrigiert) überprüft.

Ergebnisse: Der binokulare UNVA besserte sich im Median von präoperativ Jg 6 auf postoperativ Jg 2 und blieb stabil bis zur letzten Kontrolle $(87,5 \% \geq \operatorname{Jg} 2)$. Auch der unkorrigierte binokulare Intermediärvisus verbesserte sich im Mittel von präoperativ 0,8 auf 1,0 und blieb im Kontrollzeitraum ebenfalls stabil $(66,7 \%$ $\geq 1,0$ ). 12 Monate postoperativ war der unkorrigierte Fernvisus am implantierten Auge im Median 1,0 - binokular sogar 1,25 - womit der unkorrigierte Fernvisus im Vergleich zu den präoperativen Werten unverändert blieb. Auch die Refraktion blieb im Kontrollzeitraum stabil (MRSE 1 Jahr postop: 0,26 \pm 0,37 D). Weder während der Implantation, noch postoperativ kam es innerhalb des ersten postoperativen Jahres zu Komplikationen.

Schlussfolgerungen: Die Pocket-Technik zur Implantation des KAMRA ${ }^{\mathrm{TM}}$ Cornealen Inlays kann nach derzeitiger Datenlage als sicheres, effektives und potentiell reversibles chirurgisches Verfahren zur Presbyopiekorrektur bei emmetropen Patienten angesehen werden.

Schlüsselwörter: Presbyopie, Hornhautimplantat, Cornea, refraktive Chirurgie. Key words: Presbyopia, corneal inlay, cornea, refractive surgery.

29. W. Riha, A. Dexl, T. Rückl, O. Seyeddain, V. Reischl, G. Grabner (Salzburg): Subjektive und Objektive Qualität des Sehvermögens nach Implantation des KAMRA ${ }^{\mathrm{TM}}$ Corneal Inlays zur chirurgischen Behandlung der Presbyopie (Kurzvortrag) - Quality of vision in patients implanted with a small aperture optic corneal inlay

Problemstellung: Das KAMRATM Corneal Inlay (vormals AcuFocus ${ }^{\mathrm{TM}}$ Corneal Inlay, ACI) ist ein intrakorneales Implantat, welches zur chirurgischen Korrektur der Presbyopie - dem Effekt einer stenopäischen Lücke entsprechend - in die Hornhaut des nicht dominanten Auge (nach Präparation eines Flaps oder eines Pockets mit dem Femtosekundenlaser) eingesetzt wird. Im Rahmen dieser Studie erfolgte die Evaluation von Kontrastsensitivität und Patientenzufriedenheit nach KAMRA ${ }^{\mathrm{TM}}$ Implantation bei emmetropen, presbyopen Patienten.

Methode und/oder Patienten: Wir untersuchten 32 Patienten innerhalb der ersten 3 Jahre nach KAMRA ${ }^{\mathrm{TM}}$ Implantation, welche im Rahmen der FDA-Zulassungsstudie an der Universitätsaugenklinik Salzburg erfolgte. Die Kontrastsensitivität wurde mit dem Optec 6500P Vision Tester (Stereo Optical, Chicago, U.S.), unter Verwendung der F.A.C.T Charts, bei photopischen $\left(85 \mathrm{~cd} / \mathrm{m}^{2}\right)$ und mesopischen $\left(3 \mathrm{~cd} / \mathrm{m}^{2}, \pm\right.$ Glare von $\left.28 \mathrm{lux}\right)$ Bedingungen erhoben. Die Evaluation der Patientenzufriedenheit erfolgte mittels standardisierter Fragebögen.

Ergebnisse: Die binokulare Kontrastsensitivität war 1, 2 und 3 Jahre postoperativ unverändert zu den präoperativ erhobenen Werten. Die Ergebnisse am im-
Bemerkungen 
plantierten Auge zeigten eine geringe Abnahme unter allen getesteten Bedingungen. Bei der 3-Jahres Kontrolle beurteilten die Patienten ihre Nahsicht als signifikant besser $(p .<0.001)$ ohne wesentliche Verschlechterung in der Ferne. 78.1\% stuften Nachtsichtprobleme als nicht vorhanden bzw. gering ein; Halos wurden von $71.8 \%$ gar nicht bzw. kaum bemerkt. Während präoperativ $87.5 \%$ der Patienten ständig eine Lesebrille verwendeten, tat dies nach dem Eingriff niemand mehr. Befragt nach dem Grad Ihrer Zufriedenheit gaben 96.6\% an, sehr zufrieden oder einigermaßen zufrieden zu sein, daher würde auch die überwiegende Mehrheit (84.5\%) diesen Eingriff neuerlich vornehmen lassen.

Schlussfolgerungen: Es zeigte sich zwar eine Abnahme der Kontrastsensitivität am implantierten Auge, jedoch keine Verschlechterung des binokularen Befundes. Die Patienten berichteten von einer stark verminderten Abhängigkeit von Lesehilfen und gaben eine signifikante Verbesserung im Nah- sowie Intermediärbereich an. Das KAMRA ${ }^{\mathrm{TM}}$ Inlay scheint nach derzeitigem Wissenstand eine effektive und sichere Behandlungsoption zur chirurgischen Behandlung der Presbyopie zu sein.

Schlüsselwörter: Presbyopie, Hornhautimplantat.

Key words: Presbyopia, corneal implant.

30. A. Dexl, O. Seyeddain, W. Riha, M. Hohensinn, T. Rückl, V. Reischl, G. Grabner (Salzburg): Vergleich von Nahvisus und funktioneller Lesefähigkeit nach refraktiv-chirurgischer Presbyopiekorrektur (Vortrag) - Comparing near visual acuity vs. functional reading ability as primary outcome measures after presbyopic surgery

Problemstellung: Im Rahmen von klinischen Studien die sich mit der chirurgischen Korrektur der Presbyopie auseinandersetzen werden derzeit weltweit die verschiedensten Verfahren zur Erhebung des Visus im Nahbereich herangezogen. Während der Einzeloptotypen-Nahvisus lediglich eine Aussage über die Auflösungskapazität des optischen Systems ermöglicht, kann jedoch durch die Erhebung des Lesevisus ein Rückschluss auf die funktionelle Lesefähigkeit erfolgen.

Methode und/oder Patienten: Im Rahmen einer prospektiven, nicht-randomisierten, Interventionsstudie erfolgte die Implantation des KAMRA ${ }^{\mathrm{TM}}$ intrakornealen Inlays in das nicht dominante Auge von 32 natürlich emmetropen und presbyopen Patienten. Über einen mittleren Nachbeobachtungszeitraum von 36 Monaten wurde der binokulare unkorrigierte Nahvisus (OPTEC 6500P Vision Tester, mit fixer Distanz von $40 \mathrm{~cm}$ ), sowie die binokulare unkorrigierte Lesefähigkeit (Salzburg Reading Desk, mit variabler -subjektiv bester - Lesdistanz) erhoben und miteinander verglichen.

Ergebnisse: Präoperativ waren die Werte des unkorrigierten binokularen Nahbzw. Lesevisus vergleichbar $(0,39 \pm 0,12 \log$ MAR vs. 0,38 $\pm 0,13 \log \mathrm{RAD}, \mathrm{p}=0,78)$; jedoch zeigten sich im postoperativen Verlauf deutliche Unterschiede $(-0,01 \pm 0,13 \log$ MAR vs. 0,22 $\pm 0,13 \operatorname{logRAD}$ nach 36 Monaten, p.<0.001). Zusätzlich erlaubte das Salzburg Reading Desk die Erhebung weiterer Parameter, die zur Beurteilung der funktionellen Lesefähigkeit herangezogen werden können. Hierbei zeigten sich deutliche Veränderugen in der subjektiv angenehmsten (besten) Lesedistanz (prae-op: $48.1 \pm 5.5 \mathrm{~cm}$ vs. $38.0 \pm 6.0 \mathrm{~cm} 36$ Monate post-op, $\mathrm{p}<0.001$ ), des kleinst-lesbaren logarithmisch skalierten Satzes (mittlerer Anstieg von $2.6 \pm 1.3$ Sätzen, $\mathrm{p}<0.001$ ), sowie der Lesegeschwindigkeit (prae-op: $142 \pm 13$ wpm vs. $152 \pm 35$ wpm 36 Monate post-op, $\mathrm{p}=0.029$ ).

Schlussfolgerungen: Das KAMRA ${ }^{\mathrm{TM}}$ intrakorneale Inlay führt zu einer deutlichen Verbesserung sämtlicher Parameter des binokularen unkorrigierten Nahvisus bzw. der funktionellen Lesefähigkeit innerhalb eines minimalen Nachbeobachtungszeitraumes von 36 Monaten. Die Erhebung des reinen Nahvisus (mit fixer Prüfdistanz) scheint jedoch die Veränderungen der funktionellen Lesefähigkeit (mit subjektiv bester Lesedistanz) um einen Faktor von 0,26 \pm 0,12 logEinheiten zu hoch einzuschätzen. 
31. T. Rückl, A. Dexl, V. Reischl, P. Binder, G. Grabner (Salzburg): Intrastromale bogenförmige Keratotomie (ISAK) mit Hilfe des Femtosekundenlasers zu Behandlung von regulären Astigmatismen (Kurzvortrag) - Intrastromal arcuate keratotomy using a femtosecond laser for the treatment of regular corneal astigmatism

Problemstellung: Die Verwendung des Femtosekundenlasers zur Platzierung bogenförmiger Schnitte in der refraktiv-chirurgischen Therapie des kornealen Astigmatismus befindet sich derzeit in der ersten Phase der klinischen Erprobung. Durch individuelle Vorprogrammierung des Lasers ist es möglich, Inzisionen unter Aussparung sowohl des Epithels samt Bowman Membran als auch des Endothels samt Descemet Membran, also gänzlich intrastromal, präzise auszuführen. Ziel dieser Pilot-Studie ist es, die Brauchbarkeit des iFS150 Femtosekundenlaser (AMO) hinsichtlich dieser Verwendung - samt individueller Anpassung der Schnittkonfiguration (Winkel, Tiefenausdehnung und Radius) - zu analysieren.

Methode und/oder Patienten: In dieser prospektiven „Single-Center“-Studie wurden 2 Patientengruppen mit kornealem Astigmatismus (natürlicher Astigmatismus: $\mathrm{n}=13$; Astigmatismus nach Cat OP: $\mathrm{n}=3$ ) mit dem iFS-Laser behandelt. Je nach Ausmaß des Astigmatismus ( 0,75 bis 3,0 D) wurden 4 verschiedene intrastromale Behandlungsmuster - die sich in Radius, Winkel der Schnittführung sowie Tiefenausdehnung unterschieden - eingesetzt. Vor der Behandlung mit dem iFS-Laser erfolgte intraoperativ die Bestimmung der steilen Hornhautachse mittels Video-Topographie (Keratron Scout), sowie der Hornhautdicke in 4 Quadranten (mittels Ultraschall-Pachymeter). Im Rahmen der Nachkontrollen (bisher 1 Tag, 1 Woche, sowie 1, 3 und 6 Mo postoperativ) wurde neben der manifesten Refraktion die Änderung der K-Werte sowie die subjektive Patientenzufriedenheit anhand eines Fragebogens überprüft.

Ergebnisse: Bisher wurden 16 Augen mit dieser neuen Methode behandelt. Im Rahmen der operativen Eingriffe konnten alle Inzisionen komplikationslos durchgeführt werden. Bereits am ersten postoperativen Tag waren die intrastromalen Schnitte bei der Spaltlampenuntersuchung kaum mehr sichtbar, konnten jedoch mit Hilfe des Visante-OCT sicher detektiert werden. Diese ersten Beobachtungen zeigen eine Reduktion des Astigmatismus zw. 0,25 und 2,5 Dioptrien mit einer hohen Stabilität der Refraktion und Topographie im weiteren Beobachtungsverlauf. Das Ausmaß der induzierten Änderung war stark abhängig vom Grad des Schnittwinkels, wobei mit dem 30 inkliniertem Schnitt die höchsten Korrekturen erzielt wurden. Die Endothelzellzahl zeigte - verglichen zu den präoperativen Werten - keine Veränderung. Die subjektive Patientenzufriedenheit war im Allgemeinen sehr hoch.

Schlussfolgerungen: Der iFS150 Femtosekundenlaser erlaubt die Durchführung rein intrastromaler Hornhautschnitte, welche in dieser Form und Präzision aus prinzipiellen Gründen bisher nicht durch andere Verfahren, wie die konventionelle Methode mit dem Diamantmesser, erreicht werden konnten. Die ersten Ergebnisse demonstrieren die Möglichkeit einer hochpräzisen Schnittsetzung bei optimalen Sicherheitsbedingungen, da weder Epithel noch Endothel tangiert werden. Als weiterer Vorteil sind die rasche Visusstabilisierung und potentielle Flexibilität hervorzuheben.

Schlüsselwörter: Astigmatismus, Laser, Refraktive Chirurgie, Cornea. Key words: Astigmatism, laser, refractive surgery, cornea. 
32. M. Rasp, A. Dexl, M. Emesz, G. Grabner (Salzburg): Intraokularlinsen-Implantation nach Epikeratophakie - Eine retrospektive Analyse von 6 Patienten (Vortrag) - Removal of epikeratophakia lenticules and implantation of intraocular lenses - a retrospective analysis of six patients

Problemstellung: Die Epikeratophakie ist eine vor über 30 Jahren eingeführte Operationstechnik, bei welcher humanes, auf der Kryodrehbank bearbeitetes und meist lyophilisiert transportiertes Hornhaut-Gewebe eines Spenders auf der deepithelialisierten Hornhaut nach zirkulärer Keratektomie fixiert wird, um eine Veränderung der K-Werte zu erreichen, vergleichbar mit einer Kontaktlinse. Mit einer erhöhten Anzahl von Katarakt-Operationen in dieser Patientengruppe ist nun zu rechnen. In dieser retrospektiven Analyse wird das Ergebnis nach Entfernung von Epikeratophakie-Lentikeln zur Korrektur einer hochgradigen Myopie und konsekutiver IOL-Implantation an der Univ.-Augenklinik Salzburg vorgestellt.

Methode und/oder Patienten: Zwischen 1999 und 2009 stellten sich 6 Patienten nach myoper Epikeratophakie (6 Frauen, Alter 55,2 \pm 7,0 Jahre) an der Univ.-Augenklinik der PMU Salzburg aufgrund einer operationswürdigen Katarakt vor. Nach umfassender Beratung wurde im ersten Schritt die Entfernung des Lentikels durchgeführt. Nach mehreren Wochen, die Epithelheilung erfolgte immer problemlos, die zentrale Hornhaut war nach fast 20 Jahren klar, erfolgte eine Wiedervorstellung zur Durchführung einer Hornhaut-Topographie (Keratron), bzw. Biometrie (IOL-Master) um eine genaue Berechung der Intraokularlinse vorzunehmen. Die K-Werte wurden für die Kalkulation von der Topographie übernommen. Phakoemulsifikation und Hinterkammer-Linsen-Implantation waren in allen Fällen problemlos. Der bestkorrigierte Fernvisus wurde vor Lentikelentfernung sowie nach der Katarakt-OP gemessen und das sphärische Äquivalent ermittelt.

Ergebnisse: Die Operation der Epikeratophakie erfolgte zwischen 1985 und 1986 an der 2. Wiener Augenklinik zur Behandlung einer hochgradigen Myopie ( $n=9$, fecit GG). Die Zeitdauer zwischen der Fixation der Lentikel und der nachfolgenden Entfernung betrug 19,0 \pm 4,2 Jahre. Eine Phakoemulsifikation mit HKL-Implantation wurde 5,0 \pm 9,8 Monate (von 1-31 Monate) nach Lentikelentfernung durchgeführt. Die mittleren K-Werte der Hornhaut wurden vor und nach der Lentikel-Entfernung mittels Hornhaut-Topographie (Keratron) vermessen, hierbei zeigte sich ein Anstieg der K-Werte von 39,8 $\pm 3,8$ D auf 46,2 \pm 1,5 D. Die implantierten IOL's hatten eine Brechkraft von $-2,3 \pm 4,9 \mathrm{D}$ im Mittel. Der bestkorrigierte Fernvisus postoperativ betrug $0,4 \pm 0,3$, das sphärische Äquivalent lag bei $-2,6 \pm 2,4$ Dioptrien (Bereich: $-5,5$ bis $+0,75 \mathrm{D}$ ).

Schlussfolgerungen: Für ein möglichst optimales postoperatives Ergebnis bei Epikeratophakie-Patienten mit operationswürdiger Katarakt hat sich im Rahmen unserer Serie eine Wartezeit von 3-4 Monaten nach Lentikel-Entfernung bewährt. Dieses Vorgehen ermöglicht stabile Hornhaut-Verhältnisse sowie eine möglichst exakte Kalkulation der jeweiligen Intraokularlinse und damit Rehabilitation dieser Patienten.

Schlüsselwörter: Epikeratophakie, hochgradige Myopie, Lentikel-Entfernung, Katarakt-Operation.

Key words: Epikeratophakia, high myopia, lenticule-removal, cataract-surgery.

33. S. Pieh, G. Schmidinger, C. Skorpik (Wien): Korrekturmöglichkeiten höherer kornealer Aberrationen mittels eines Excimerlasers der neuesten Generation (Vortrag) - Reducing corneal aberrations of higher orders using an Excimer laser of the newest generation

Problemstellung: Verbesserungsmöglichkeiten der Abbildungsqualität von Hornhäuten unter Verwendung eines Excimerlasers mit einer Spotgröße von 0,54 mm.

Methode und/oder Patienten: An 36 Patienten nach einer perforierenden Keratoplastik, lamellären Keratoplastik, nach Verletzungen sowie oberflächlichen Entzündungen mit einem sphärischem Äquivalent von 0 bis - 9 D und einem 
Hornhautzylinder bis zu 10 D wurde eine PRK mit Mitomycin durchgeführt. Die Erstellung des Abtragungsprofiles erfolgte anhand der ermittelten kornealen Wellenfront sowie der subjektiven Refraktion. Alle Behandlungen wurden mit den Amaris Excimer Laser (Schwind) durchgeführt.

Ergebnisse: Alle Patienten wurden nach einem Jahr nachkontrolliert. Die Sehschärfe mit bester Korrektur änderte sich von 0,59 $\pm 0,22$ auf 0,67 $\pm 0,27$. Der der RMS Fehler der höheren Aberrationen verbesserte sich von $2.36 \mu \mathrm{m} \pm 1,05$ auf $1,48 \mu \mathrm{m} \pm 0,79$ (minus 37,7\%), der PV-Wert der höheren Aberrationen von 15,98 $\mu \mathrm{m}$ $\pm 7,32$ auf $10,34 \mu \mathrm{m} \pm 7,22$ jeweils bezogen auf einen $6 \mathrm{~mm}$ Durchmesser. Es bestand eine Korrelation zwischen dem Ausgangs RMS - Fehler und dem Ergebnis. Deutliche Visusverbesserungen zeigten sich erst wenn der postoperative RMS Fehler höherer Ordnung unter $1 \mu \mathrm{m}$ lag.

Schlussfolgerungen: Oberflächliche Excimer-Laserbehandlungen stellen bei entsprechenden Voraussetzungen eine wichtige Methode zur Reduktion von kornealen Abbildungsfehlern höherer Ordnung dar.

Schlüsselwörter: Excimer laser, Mitomycin, Keratoplastik, korneale Wellenfront.

Key words: Excimerlaser, mitomycin, keratoplasty, corneal wavefront.

34. J. Ruckhofer, J. Stoiber, T. Rückl, V. Reischl, G. Grabner (Salzburg): Torische Artiflex zur Korrektur von myopem Astigmatismus (Vortrag) - Toric Artiflex phakic intraocular lens for the correction of myopic astigmatism

Problemstellung: Die Korrektur von myopen Astigmatismus mittels Laserabalation ist durch die Hornhautdicke limitiert. Eine Alternative stellen phake Intraokularlinsen (IOL) dar. Bereits seit über 20 Jahren gibt es die irisfixierte phake IOL (Artisan, Verisyse) aus PMMA. Die Verwendung von Silikon anstatt PMMA für die Optik erlaubt kleinere Inzisionen (Artiflex, Veriflex). Seit 2009 ist die torische Version der flexiblen Linse (torische Artiflex) zur Korrektur von myopem Astigmatismus erhältlich.

Methode und/oder Patienten: In 40 Augen von 22 Patienten wurde eine torische Artiflex implantiert. Das präoperative sphärische Äquivalent der manifesten Refraktion (MRSE) lag bei $-7,81 \mathrm{D} \pm 2,18 \mathrm{D}(-2,63$ bis $-13,0)$, der präoperative Astigmatismus bei $1,79 \mathrm{D} \pm 0,98 \mathrm{D}(1,0$ bis 5,0). Der Nachkontrollzeitraum war 3 bis 12 Monate.

Ergebnisse: Die Implantation der torischen Artiflex erfolgte ohne intraoperative Komplikationen. Zum Zeitpunkt der letzten Kontrolle lag das MRSE im Mittel bei $-0,04 \mathrm{D} \pm 0,21 \mathrm{D}$ (von $-0,50$ bis 0,75 ), der refraktive Zylinder im Mittel bei 0,14 D \pm $0,27 \mathrm{D}(0$ bis 1,0$)$. Der unkorrigierte Fernvisus war in $88 \%$ gleich oder besser als 1,0 , beim bestkorrigierten Fernvisus kam es zu keinem Verlust von 2 oder mehr Zeilen. In 3 Fällen kam es zu einer verstärkten Fibrinreaktion postoperativ, eine temporäre Druckerhöhung wurde in 2 Augen beobachtet.

Schlussfolgerungen: Die Implantation einer torischen Artiflex ist eine sichere und genaue Methode zur Korrektur von myopem Astigmatismus. Wie bei anderen phaken Intraokularlinsen sind auch hier längere Nachkontrollen notwendig.

Schlüsselwörter: Phake Intraokularlinsen, torische Artiflex, Myoper Astigmatismus.

Key words: Phakic intraokular lens, toric Artiflex, myopic astigmatism.
Bemerkungen 
35. B. Lübke, S. Höhler (Linz): Erste Erfahrungen mit dem European Registry Bemerkungen of Quality Outcomes for Cataract and Refractive Surgery (EUREQUO) in der Augenklinik der Barmherzigen Brüder in Linz. (Kurzvortrag) - First experiences with the European Registry of Quality Outcomes for Cataract and Refractive Surgery (EUREQUO) in the ophthalmic hospital of St. John of God in Linz

Problemstellung: Die Sicherung der ärztlichen Qualität rückt im Gesundheitssystem zunehmend in den Blickpunkt, so dass die Kenntnis, Überprüfung und Sicherung eigener Daten immer mehr an Bedeutung gewinnt. Die Europäische Union finanziert durch Fördermittel im Rahmen des Public Health Programms das European Registry of Quality Outcomes for Cataract and Refractive Surgery (EUREQUO), wobei die ESCRS und 12 Nationale Ophthalmologische Gesellschaften als Partner auftreten. Ziel dieses Vortrages ist es, dieses Projekt und unsere Daten aus der Augenklinik der Barmherzigen Brüder in Linz vorzustellen sowie über unsere Erfahrungen mit dem Projekt zu berichten.

Methode und/oder Patienten: Das European Registry of Quality Outcomes for Cataract and Refractive Surgery (EUREQUO) ist ein Europäisches Qualitätsregister für Ergebnisse von Katarakt und refraktiven Eingriffen. Mit dieser webbasierten Qualitätssicherungsdatenbank sammeln Ophthalmochirurgen unterstützt durch einen Registry Manager (für Deutschland und Österreich: Frau Höhler) anonymisierte Patientendaten. Es handelt sich um das größte IT Projekt in der internationalen Ophthalmologie. Es wurde als dreijähriges Projekt von der ESCRS und von 12 europäischen ophthalmochirurgischen Fachgesellschaften (für Deutschland und Österreich: DGII) initiiert und von der EU und der ESCRS kofinanziert, wobei die ESCRS das Projekt auch nach Februar 2011 weiter unterstützen wird. Das Datenregister können Augenärzte in Österreich und Deutschland seit März 2010 nutzen, wobei eine Registrierung jederzeit möglich ist.

Ergebnisse: Die Daten werden anonymisiert ohne Patientennamen nur mit einer von der Klinik bzw. dem Zentrum bestimmten Patientennummer gespeichert. Erfasst werden Patientennummer, Geburtsjahr, Geschlecht, ethnische Zugehörigkeit, ASA-Klassifikation und prä-/intraoperative ophthalmologische Daten wie Visus, Refraktion, Zielrefraktion, Keratometerwerte, Augenkomorbidität, Operationsdatum, Anästhesie-/Operationstechnik, Art der Intraokularlinse, intraoperative Komplikationen und das Operationssetting (ambulant oder stationär). Bei der fakultativ postoperativen Datenerhebung werden Untersuchungstag, unkorrigierter Visus, Komplikationen und ggf. der korrigierte Visus mit Refraktions- und Keratometerwerten erhoben. Zum Thema Kataraktchirurgie wurden bis zum 1.12.2010 europaweit 102107 Datensätze gespeichert, davon 3060 aus Österreich und davon 3006 aus der Augenklinik der Barmherzigen Brüder in Linz.

Schlussfolgerungen: Das vorgestellte Qualitätssicherungsprojekt ermöglicht dem operierenden Augenarzt eine kontinuierliche Qualitätssicherung, wie es Bestandteil aller Qualitätsmanagementsysteme ist. Dem einzelnen Augenarzt bzw. dem teilnehmendem Zentrum bietet sich die Möglichkeit, die eigenen Ergebnisse mit den Summenergebnissen aller teilnehmenden Zentren im eigenen Land bzw. europaweit zu vergleichen. Ziel ist es das Interesse und Bewusstsein für Qualitätssicherheit zu wecken und die Behandlungs- und Versorgungsstandards im refraktiven und Kataraktbereich zu verbessern. Hauptanliegen ist die Entwicklung von evidenzbasierten europäischen Qualitätsrichtlinien.

Schlüsselwörter: European Registry of Quality Outcomes for Cataract and Refractive Surgery (EUREQUO), Qualitätssicherung, Qualitätsmanagement, Kataraktchirurgie, refraktive Chirurgie, Europäische Union (EU), European Society of Cataract and Refractive Surgeons (ESCRS).

Key words: European Registry of Quality Outcomes for Cataract and Refractive Surgery (EUREQUO), quality assurance, quality management, cataract surgery, refractive surgery, European Union (EU), European Society for Cataract and Refractive Surgeons (ESCRS). 
36. V. Reischl, T. Rückl, M. Hohensinn, A. Dexl, G. Grabner (Salzburg): Ein intBemerkungen raoperatives Navigationssystem zur Implantation von Intraokularlinsen (Surgery Guidence System, SMI) (Kurzvortrag) - Implantation of intraocularlenses with the Surgery Guidence System, SMI

Problemstellung: Bestimmung der Genauigkeit der Implantationsachse torischer Intraokularlinsen mit Hilfe eines innovativen eye-tracking Navigationssystems der Firma SMI (Berlin). Gerade bei torischen intraokularen Linsen (tIOL) ist eine exakte Positionierung von großer Bedeutung, da bereits eine Abweichung von 1 einen dreiprozentigen Verlust der Zylinderwirkung mit sich bringt. Nicht nur aufgrund von Unterschieden der manuellen Markierungstechniken, sondern auch durch die statische Cyclotorsion, welche Werte bis zu 11 annehmen kann, wird die intraoperative Achsenausrichtung einer t-IOL deutlich erschwert und weniger kalkulierbar.

Methode und/oder Patienten: Das Surgery Guidence System besteht aus einer „Reference Unit“ (RE) und dem „Surgery Pilot“ (SP), der ein intraoperatives Navigationssystem mit eye-tracking-Funktion darstellt. Präoperativ misst die RE am sitzenden Patienten die Hauptzylinderachse sowie die Höhe des Astigmatismus. Der SP gleicht intraoperativ am liegenden Patienten das live-Bild des OPMikroskopes an das präoperative Referenzbild an, indem er sich an Gefäß-\& Irisstrukturen orientiert und gleichzeitig den Cyclotorsionsgrad berechnet. Wesentliche OP-Schritte, wie Inzision, Rhexis und Achsenpositionierung können am Bildschirm des SP vom Operateur geplant werden. Diese werden dann als Overlays intraoperativ entsprechend eingeblendet. Zur Überprüfung der Genauigkeit dieses Systems wurde die seit 15 Jahren an der Univ.-Augenklinik Salzburg verwendete Methode der prä- und intraoperativen Keratron Scout-Messungen herangezogen.

Ergebnisse: Erste Ergebnisse an 25 Augen zeigen eine sehr gute Vergleichbarkeit des Cyclotorsionsgrades beider Messsysteme. Sie unterscheiden sich nicht signifikant, wobei die Handhabung bei niedrigen Hornhautastigmatismen mit dem neueren System wesentlich einfacher ist. Entsprechend gering ist der postoperative refraktive Astigmatismus und die Patientenzufriedenheit sehr hoch. Das Verfahren wird im Detail vorgestellt.

Schlussfolgerungen: Mit Hilfe des Surgery Guidence Systems wird die Positionierung von torischen IOLs wesentlich erleichtert. In naher Zukunft wird beim SG 5000 das Overlay auch in das Okular des Operationsmikroskopes eingeblendet werden können. Dies wird dem Operateur neben einer kürzeren Operationszeit auch eine noch komfortablere Implantation der Linsen ermöglichen.

Schlüsselwörter: Astigmatismus, torische IOL-Implantation, intraoperative Positionierung, statische Cyclotorsion.

Key words: Astigmatism, toric IOL implantation, positioning of the axis, static cyclotorsion.

37. M. Hohensinn, A. Dexl, V. Reischl, T. Rückl, M. Rasp, G. Grabner (Salzburg): Vorhersagbarkeit der postoperativen Refraktion durch Z CALC bei der Verwendung der bitorischen, asphärischen, monofokalen IOL *AT.Comfort 646TLC (Vortrag) - Predictability of postoperative refraction using Z CALC and the monofocal, aspheric, bitoric IOL *AT.Comfort 646TLC

Problemstellung: Bei den meisten Patienten kann durch die Implantation rotationssymmetrischer Intraokularlinsen (IOL) im Rahmen einer Cataractoperation ein postoperativ gutes visuelles Ergebnis erzielt werden. Bei höheren - bereits präoperativ bekannten Hornhautastigmatismen - ist damit jedoch keine optimale refraktive Versorgung möglich. Neben anderen, etwa cornealen, Methoden der Astigmatismuskorrektur werden zunehmend torische IOLs zur Lösung des Problems verwendet, da dadurch die Risken oberflächlicher inzisionaler Chirurgie vermieden und die Patientenbelastung reduziert werden können. 
Methode und/oder Patienten: Im Rahmen einer multizentrischen Studie wurde an der Univ.-Augenklinik Salzburg die bitorische, asphärische, monofokale Intraokularlinse *AT.Comfort 646TLC (= AT TORBI 709M, Carl Zeiss Meditec) zur Korrektur präexistenter Hornhautastigmatismen im Rahmen von Cataract-Operationen bei 28 Augen (28 Patienten) implantiert. Die Berechnung der IOL-Stärke und Vorhersage der postoperativ zu erwartenden Refraktion erfolgte präoperativ nach den K-Werten des IOL-Masters bzw. topographischer Analyse mit Z CALC, die genaue Achsenlage wird intraoperativ am liegenden Patienten mittels Topographie (Keratron Scout) kontrolliert und modifiziert. Die postoperativen Untersuchungen erfolgten 1-8 Tage sowie 6-12 Wochen nach Implantation und umfassen u. a. die Bestimmung der Refraktion und Sehschärfe, Bestimmung der postoperativen Achse des IOL-Torus, Topographie sowie eine Messung der Vorderkammertiefe.

Ergebnisse: Bei allen inkludierten Patienten konnte die Intraokularlinse problemlos implantiert werden. Der IOL-Torus betrug zwischen 1,0 und 9,5 D. Bei den Nachkontrollen betrug der mittlere postoperative Restastigmatismus 0,2 D (Bereich: 0 D - 1,25 D). Im Vergleich der intraoperativen und postoperativen Fotodokumentation zeigten sich die implantierten Linsen während des gesamten Nachbeobachtungszeitraums überwiegend rotations- und lagestabil. Bei einer Patientin war eine Nachrotation nötig, sie hatte danach keinen Restastigmatismus mehr der einer Korrektur bedurfte. Durch den bei allen Patienten gewählten corneo-skleralen Zugang fand sich bei der Gegenüberstellung von präoperativen und postoperativen Topographien ein operationsinduzierter Astigmatismus unter $0,25 \mathrm{D}$.

Schlussfolgerungen: Für Cataractoperationen bei zusätzlichem, präoperativ bekanntem Hornhautastigmatismus ist die Verwendung torischer Intraokularlinsen eine vielversprechende Option, da einfach durchführbar, komplikationsarm und für den Patienten nicht zusätzlich belastend. Das verwendete Programm zur Berechnung der Torus- und Linsenstärke ermöglicht eine gute Vorhersagbarkeit der postoperativen Refraktion. Zur genauen Ausrichtung der Achsenlage durch den Chirurgen scheint die Durchführung einer intraoperativen Hornhauttopographie der derzeit zielführendste Ansatz zu sein. Weitere Studien zur Erhärtung der bisherigen Ergebnisse sind erforderlich.

Schlüsselwörter: Torische IOL, Topographie, Cataract, Stabilität, operationsinduzierter Astigmatismus.

Key words: Toric IOL, topography, cataract, stability, wave front, surgical induced astigmatism.

38. A. Bachernegg, M. Rasp, W. Riha, O. Seyeddain, M. Hohensinn, G. Grabner, A. Dexl (Salzburg): Die visuelle Performance der asphärischen XL Stabi ZO im Vergleich zur sphärischen XL Stabi SKY - 1 Jahres- Ergebnisse einer prospektiven, randomisierten, kontrollierten, klinischen Studie (Kurzvortrag) - Performance of the XL Stabi ZO aspheric intraocular lens vs. the spherical XL Stabi Sky intraocular lens - 1 year results of a randomized controlled clinical trial

Problemstellung: Derzeit werden von vielen Ophthalmochirurgen vorwiegend asphärische Intraokularlinsen (IOLs) implantiert, da diese (in Abhängigkeit von der Pupillenweite) die durch sphärische Optiken hervorgerufenen Abbildungsfehler vermindern können. Um diesen potentiellen Vorteil derartiger Konzepte zu überprüfen, wurde im Rahmen dieser Studie die relevanten Visus-Parameter der asphärischen XL Stabi ZO (ZO, Carl Zeiss Meditec SAS) mit ihrem sphärischen Pendant - der XL Stabi Sky (SKY, Carl Zeiss Meditec SAS) - verglichen.

Methode und/oder Patienten: Diese monozentrische, prospektive, randomisierte, kontrollierte und Untersucher-verblindete Pilotstudie umfasste an der Univ.Augenklinik Salzburg 40 Augen von 40 Patienten (20 ZO, 20 SKY). Mittels eines Hartmann-Shack Aberrometers (WASCA) wurde die „Modulation-Transfer“Funktion (MTF) der Aberrationen höherer Ordnung, sowie der sphärischen Aberration Z4,0 innerhalb des ersten postoperativen Jahres erhoben und gruppen-
Bemerkungen 
weise für theoretische Pupillenweiten von 3,0 und 4,5 mm verglichen. 1, 3 und 12 Monate nach erfolgter Katarakt-Operation erfolgte zusätzlich die Überprüfung der postoperativen Sehschärfe, Refraktion, Genauigkeit der Berechnung der Linsenstärke, sowie der mesopischen und photopischen Kontrastsensitivität mit dem OPTEC 6500P Vision Tester. Mittels Defokus-Kurven wurde zusätzlich die Schärfentiefen verglichen.

Ergebnisse: 12 Monate postoperativ zeigte die MTF-Messung der sphärischen Aberration einen statistisch signifikanten (p.<0.05) Vorteil der asphärischen ZO. Zusätzlich waren auch die Aberrationen höherer Ordnung (zumindest für einige Ortsfrequenzen), sowie die mesophische Kontrastintensivität in der asphärischen Gruppe siginfikant besser $(\mathrm{p}<0.05)$. Keine statistisch signifikanten Unterschiede zwischen den Gruppen konnten für die photopische Kontrastintensiviät, Sehschärfe, Refraktion und Sehschärfentiefe nachgewiesen werden.

Schlussfolgerungen: Die asphärische ZO bietet eine statistisch signifikante Verminderung der sphärischen Aberration, und ist vor allem bei Pupillendurchmessern $\geq 4,5$ mm dem sphärischen Pendant vorzuziehen. Gleichzeitig führt die Implantation der asphärischen $\mathrm{ZO}$ zu keiner (zumindest theoretisch erwarteten) Verminderung der Schärfentiefe. Um diese Ergebnisse weiter zu erhärten ist derzeit eine Nachfolge-Studie mit mehr Studienteilnehmern in Planung.

Schlüsselwörter: IOL, torische Linsen.

Key words: IOL, toric lens.

39. V. Reischl, T. Rückl, M. Rasp, W. Riha, G. Nix, A. Dexl, G. Grabner (Salzburg): Lesefähigkeit, Kontrastsehen und Patientenzufriedenheit nach Cataractoperation - Multifokallinse versus Monofokallinse (Kurzvortrag) - Reading ability, acuity of contrast vision and patient's satisfaction after cataract surgery - multifocal lens versus monofocal lens

Problemstellung: Um zu prüfen, in wie ferne die Brillenunabhängigkeit nach der Cataract-Operation in Zusammenhang mit dem gewählten Linsendesign steht, werden vier verschiedene intraokulare Linsentypen und -designs miteinander verglichen. In bisherigen vorliegenden Studien wurde der direkte Vergleich von multifokalen mit monofokalen Intraokularlinsen (IOL) eher vernachlässigt. In dieser prospektiven, randomisierten, Untersucher verblindeten Multicenterstudie wurden Multifokallinsen (MIOL) unterschiedlichen Linsendesigns und eine Monofakllinse miteinander verglichen.

Methode und/oder Patienten: In beiden Zentren (Alicante/Spanien und Salzburg/Österreich) wurden insgesamt 240 Patienten inkludiert, 30 pro Studienarm pro Zentrum, und postoperativ über einen Zeitraum von 12 Monaten nachbeobachtet. Nach dem Zufallsprinzip wurden den Patienten folgende Linsen zugeteilt: AT.Smart 46S (monofokale Standardlinse), Rezoom (refraktive Multifokallinse), AT.Lisa 366D (diffraktive MIOL) und ReStor SN6AD3 (apodisierte diffraktive MIOL). Nach bilateraler Phakoemulsifikation (jeweils ein Operateur pro Zentrum) und Linsenimplantation wurden die Parameter der Lesefähigkeit, das Kontrastsehen und die Patientenzufriedenheit nach 3, 6 und 12 Monaten untersucht. Der Lesevisus (logRAD und „Wörter pro Minute“) wurden mit Hilfe des Salzburg Reading Desks (SRD) ermittelt, das Kontrastsehen wurde mit dem Optec 6500 P Vision Tester geprüft und die Patientenzufriedenheit mittels Fragebogen evaluiert.

Ergebnisse: Emmetropie wurde in allen vier Gruppen erreicht $( \pm 0,5)$. Das beste Ergebnis in Bezug auf die unkorrigierte Lesefähigkeit wurde mit der AT.Lisa (0,26 logRAD) erreicht, gefolgt von der ReStor Linse (0,28 logRAD). Die Patienten mit Rezoom-Implantaten erreichten im Mittel geringere Werte (0,42 logRAD) und die Lesefähigkeit mit der monofokalen AT.Smart betrug im Schnitt 0,5 logRAD.

Schlussfolgerungen: AT.Lisa und ReStor IOLs bieten eine gute Leseschärfe bei nur gering reduzierter Kontrastsensitivität. Rezoom und monofokale IOLs fielen 
bei der Leseschärfe signifikant ab. Fast alle Patienten waren sehr zufrieden mit ihren individuellen Ergebnissen. Besonders sollte auf eine gezielte Patientenselektion und -führung geachtet werden und es muss zuverlässig bei der Erstuntersuchung und -beratung auf eine mögliche Adaptionszeit von einigen wenigen Monaten aufmerksam gemacht werden.

Schlüsselwörter: Brillenunabhängigkeit, Multifokallinse, Monofokallinse.

Key words: Life without glasses, multifocal lens, monofocal lens.

40. G. Kahraman, N. El-Shammah, K. Nigl, M. Amon (Wien): Sulcoflex, ein additives Linsensystem zur Korrektur der pseudophaken Ametropie im mit Silikonöl gefüllten Auge. (Kurzvortrag) - Sulcoflex: A supplementary IOL for the correction of pseudophakic ametropia in the Silicone-oil filled eye.

Problemstellung: Im mit Silikonöl gefüllten Auge entsteht eine Refraktionsänderung. Additive Linsensysteme, sind ein etabliertes Verfahren zur Korrektur der pseudophaken Ametropie, und stellen eine interessante Option in diesen Fällen dar. Eine additive Sulcuslinse kann je nach Bedarf eingesetzt, entfernt oder ausgetauscht werden.

Methode und/oder Patienten: Bei Patienten mit einem mit Silikonöl gefüllten Auge und dadurch entstandener postoperativer Ametropie wurde eine Rayner Sulcoflex IOL in den Sulcus ciliaris implantiert. Bei diesem Linsentyp handelt sich um ein „single piece“ Implantat aus hydrophilem Akryl. Optik- und Haptikkanten sind abgerundet. Die asphärische Optik („linsenneutral“) hat einen Durchmesser von 6,5 mm und ist konvex/konkav, um eine zentrale Kontaktzone mit der zweiten Linsenoptik zu vermeiden. Postoperativ wurden UCVA und BCVA für Ferne und Nähe sowie der Augendruck bestimmt. Position und Rotation der IOLs wurden unmittelbar postoperativ und zu allen Kontrollterminen dokumentiert. Zusätzlich wurden Scheimpflug Bilder aufgenommen und der Inflammationsgrad beurteilt.

Ergebnisse: Bisher wurden insgesamt 4 Augen operiert. Alle Augen erhielten eine monofokale Version der Sulcoflex IOL. Bei 3 Augen bestand schon eine YAG-Laser Kapsulotomie. Das Durchschnittsalter der Patienten betrug 64 Jahre. Die präoperative Ametropie lag zwischen +8 Dioptrien und +3 Dioptrien. Der bisherige Nachbeobachtungszeitraum beträgt 5 Monate. Es kam zu keinerlei intra- und postoperativen Komplikationen. Der Augendruck war bei allen Kontrollen im Normbereich. Die Patienten hatten nach der Operation keine aniseikonischen Beschwerden. Wir haben kein Silikonöl in der Vorderkammer beobachtet.

Schlussfolgerungen: Nach nunmehr fast 6 Monaten Nachbeobachtung kann gesagt werden, dass die Sulcoflex IOL im silikonölgefüllten Auge gut toleriert wird. Die Implantation einer additiven IOL ist weniger traumatisierend als der Tausch einer IOL. SulcoflexR stellt ein interessantes, vielseitig einsetzbares und reversibles Linsenkonzept auch für diesen Indikationsbereich dar.

Schlüsselwörter: Sulcoflex, Add-on IOL, Silikonöl, pseudophaken Ametropie.

Key words: Sulcoflex, Piggyback IOL, pseudophakic ametropia, supplementary IOL.

41. D. Ivastinovic, C. Schwab, A. Borkenstein, E. Lackner, A. Wedrich, M. Velikay-Parel (Graz): Veränderungen der Glaskörpergrenzschicht nach Phakoemulsifikation (Vortrag) - Changes of vitreoretinal interface after phacoemulsification

Problemstellung: Nach einer Phakoemulsifikation ist das Risiko einer rhegmatogenen Netzhautabhebung erhöht. Die ursächlichen vitreoretinalen Traktionen kommen durch die inkomplette Glaskörperabhebung zustande. Ein zweites Merkmal dieser sogenannten anomalen Glaskörperabhebung ist die Vitreoschisis. Bisher wurde die Glaskörperabhebung mittels Biomikroskopie und Ultra- 
schall diagnostiziert. Die optische Kohärenztomographie (OCT) bietet eine deutlich bessere Abbildung des vitreoretinalen Interface und erlaubt somit die Feststellung der frühesten Formen der Glaskörperabhebung. Das Ziel unserer Studie ist mittels OCT die Glaskörperabhebungsrate nach der Phakoemulsifikation zu evaluieren.

Methode und/oder Patienten: Es handelt sich um eine prospektive Studie bei der Patienten mit beidseitiger Katarakt mittels OCT und B-scan-Sonographie untersucht wurden. Die Einschlusskriterien waren anliegender Glaskörper an dem für die Operation vorgesehenen Auge und eine komplikationslose Phakoemulsifikation. Ausgeschlossen wurden Patienten mit einer Makulopathie und diabetischer Retinopathie. Die Patienten, die die Einschlusskriterien erfüllt hatten, wurden am ersten postoperativen Tag, 1 Monat und 3 Monate nach der Operation an beiden Augen mittels OCT und Sonographie untersucht. Kontralaterale Augen mit einem anliegenden Glaskörper dienten als Kontrollaugen.

Ergebnisse: Insgesamt wurden 526 Augen von 263 Patienten untersucht. 56 Patienten haben die Einschlusskriterien erfüllt. 39 Kontrollaugen konnten rekrutiert werden. Am ersten postoperativen Tag wurden keine Veränderungen des vitreoretinalen Interface festgestellt. Nach 1 Monat wurde eine komplette oder inkomplette Glaskörperabhebung bei 18 Studienaugen (32,1\%) bzw. einem Kontrollauge $(2,6 \%)$ festgestellt ( $\mathrm{p} .<0.001)$. Nach 3 Monaten betrug die Prävalenz der kompletten oder inkompletten Glaskörperabhebung bei den Studienaugen 42.8\% ( $n=24)$ und $7.7 \%(\mathrm{n}=3)$ bei den Kontrollaugen $(\mathrm{p}<0.001)$. Vitreoschisis wurde bei 17 der Studienaugen (70.8\%) und bei keinem Kontrollauge beobachtet. Während des Nachbeobachtungszeitraums berichtete eine Patientin über Photopsien. Vitramakuläres Traktionssyndrom oder rhegmatogene Netzhautabhebung wurde bei keinem Patienten beobachtet.

Schlussfolgerungen: Die Ergebnisse zeigen, dass die Phakoemulsifikation zu einer deutlichen Beschleunigung der Glaskörperabhebung innerhalb der ersten 3 postoperativen Monate führt. Mittels OCT lässt sich neben den Frühstadien der Glaskörperabhebung auch eine Vitreoschisis feststellen. Die relativ hohe Vitreoschisisrate bei den Studienaugen deutet auf eine anomale Glaskörperabhebung hin. Nichtsdestotrotz wurden außer Photopsien bei einer Patientin keine korrespondierenden Komplikationen beobachtet. Allerdings würde ein längerer Nachbeobachtungszeitraum konklusivere Schlüsse über die Komplikationsrate erlauben.

Schlüsselwörter: Glaskörperabhebung, Phakoemulsifikation.

Key words: Posterior vitreous detachment, phacoemulsification.

42. N. Hirnschall, A. Crnej, V. Gangwani, O. Findl (Wien): Methoden zur Quantifizierung von Nachstar (Vortrag) - Methods to quantify PCO using forward and backward scatter of light

Problemstellung: Im Rahmen der Studie wurden Methoden zur Nachstar-Quantifizierung evaluiert.

Methode und/oder Patienten: Patienten mit regeneratorischem Nachstar und geplanter Nd:YAG Laser Therapie wurden in die Studie eingeschlossen. Mittels ETDRS und Pelli Robson Tafeln (beide Precision Vision) wurde die Sehleistung vor der Laser Therapie überprüft. Streulicht (C-Quant, Oculus) und „point spread function“ (OQAS, Visiometrics) Messungen, und weiters Scheimpflug (Pentacam HR, Oculus) und Retroilluminations Bilder wurden vor und nach der Lasertherapie aufgenommen. Zusätzlich wurden Symptome, wie Blendungserscheinungen anamnestisch erfasst.

Ergebnisse: 50 Augen von 50 Patienten wurden inkludiert. Durchschnittlich konnten die Patienten vor der Lasertherapie 22.4 letters (SD: 10.8) auf den ETDRS und 22.7 letters auf den Pelli Robson Tafeln (SD: 8.4) lesen. Keine der evaluierten Messmethoden zeigte eine Korrelation größer als r2 $>0.2$ zur Sehleistung der Patienten vor der Therapie. Nach der Nd:YAG Laser Behandlung blieben die CQuant Messungen unverändert (Diff: $-0.1 \log (\mathrm{s})$, SD: $0.2, \mathrm{p}>0.05)$, bei den OQAS
Bemerkungen 
Messungen war die Differenz signifikant (Diff: -4.4; SD: 2.2; $\mathrm{p}$.

Schlussfolgerungen: Die Korrelation zwischen Sehleistung und objektiver Nachstar Messung ist als moderat zu bewerten.

Schlüsselwörter: Nachstar, PCO, Streulicht, Nd:YAG laser, C-Quant, OQUAS, Pentacam, Retroillumination Foto.

Key words: After-cataract, PCO, stray light, Nd:YAG laser, C-Quant, OQUAS, pentacam, retroillumination photograph.

KAFFEEPAUSE 09:53 - 10:30

$10: 30$

VOLLVERSAMMLUNG DER ÖSTERREICHISCHEN OPHTHALMOLOGISCHEN GESELLSCHAFT

Europahaus, Saal Europa

14:00 PRIMARÄRZTESITZUNG

Hotel Neuhaus

15:00

3. WISSENSCHAFTLICHE SITZUNG

Europahaus, Saal Europa

NETZHAUT 1

Vorsitz: M. Velikay-Pavel, U. Schmidt-Erfurth

15:00

43. U. Schmidt-Erfurth, C. Simader, C. Kiss, Q. Nguyen (Wien): Resultate der Phase III -Studie zur Therapie der neovaskulären AMD mit VEGF trap: VIEW 1/2 (Vortrag) - Randomized, Double Masked, Active-Controlled Phase 3 Trial of the Efficacy and Safety of Intravitreal VEGF Trap-Eye in wet AMD: 1-yr results from the VIEW-1 and VIEW-2 studies

Problemstellung: Randomisierte, doppelt maskierte, aktive-kontrollierte Phase 3 Studie zur Wirksamkeit und Nebenwirkungsfreiheit von intravitrealem VEGF Trap-Eye (VTE) bei exsudativer Alters-bezogener Makuladegeneration (AMD) im Vergleich zu Ranibizumab: Einjahres-Resultate von VIEW-1 und VIEW-2.

Methode und/oder Patienten: In zwei parallele klinische Studien wurden insgesamt 2457 Patienten inkludiert: 1217 in VIEW-1 (Zentren in den USA) und 1240 in VIEW-2 (Zentren in Südamerika, Europa, Asien-Pazifik). Die Studienteilnehmer wurden randomisiert zu VTE mit 0,5 mg monatlich (0.5q4wk), $2 \mathrm{mg}$ jeden Monat (2q4wk), 2 mg jeden zweiten Monat (2q8wk) nach einer initialen 3 monatlichen Dosis, oder Ranibizumab 0,5 mg kontinuierlich monatlich (Rq4wk). Primärer Endpunkt war der proportionale Anteil von Patienten mit Vermeidung eines moderaten Visusverlustes von.

Ergebnisse: In VIEW-1 lag der Anteil der Patienten mit stabilem Visus nach 52 Wochen bei $94,4 \%$, 95,9\%, 95,1\%, und 95,1\% in den Gruppen mit Rq4wk, 0.5q4wk, 2q4wk, and 2q8wk. In VIEW-2 waren die korrespondierenden Werte in den Vergleichsgruppen 94,4\%, 96,3\%, 95,6\%, und 95,6\%. Damit zeigten alle VTE-Gruppen eine statistisch signifikante Non-Inferiorität (Non-inferiority Margin von 10\%) im Vergleich zu Ranibizumab. In VIEW-1 lag der mittlere Visusanstieg bei in ETDRS-Werten für Rq4wk, 0.5q4wk, 2q4wk and 2q8wk bei 8,1, 6,9, 10,9, und 7,9 Buchstaben, dabei erwies sich VTE mit 2,0 mg monatlich als besser im Vergleich zu monatlichem Ranibizumab (P.<0.01). In VIEW-2 zeigte sich ein Visusgewinn von 9.4, 9.7, 7.6 und 8.9 Buchstaben ohne signifikanten Unterschied zwischen den verschiedenen VTE-Gruppen und Ranibizuamab. Die Inzidenz von okulären und systematischen Adverse Events (AEs) war nicht signifikant.

Schlussfolgerungen: Die intravitreale Behandlung mit VEGF Trap-Eye war der kontinuierlichen monatlichen Therapie mit Ranibizumab nicht unterlegen. Eine nur zweimonatige Gabe von VEGF Trap-Eye war ebenso erfolgreich wie eine 
häufigere monatliche Verabreichung von Ranibizumab. Die Substanz war außerdem gut verträglich und zeigte ein hervorragendes Sicherheitsprofil. VEGF Trap-Eye bietet damit ein neues Therapie-Konzept im Management der exsudativen AMD mit einer reduzierten zwei-monatlichen Behandlungs-Strategie und reduziert damit den Behandlungsaufwand sowohl für Patienten als auch für Augenärzte erheblich. Die Studie wird derzeit im zweiten Jahr fortgeführt.

Schlüsselwörter: AMD intravitreale Therapie VEGF-Trap.

Key words: AMD intravitreal therapy VEGF-Trap.

44. S. Hagen, S. Ansari-Shahrezaei, E. Smretschnig, S. Moussa, I. Krebs, S. Binder (Wien): Retinale Sensitivität nach Photodynamischer Therapie mit reduzierter Lichtdosis bei akuter Chorioretinpathia centralis serosa (Vortrag) - Retinal sensitivity after reduced fluence photodynamic therapy in acute central serous chorioretinopathy

Problemstellung: In einigen Studien hat sich die photodynamische Therapie (PDT) mit Verteporfin (Visudyne ${ }^{\circledR}$, Novartis Pharma) als wirksame Behandlung der Chorioretinpathia centralis serosa (CCS) erwiesen. In der vorliegenden Studie untersuchen wir die retinale Sensitivität und die Fixationsstabilität vor und nach erfolgter PDT mit reduzierter Lichtdosis bei der akuten Form der CCS.

Methode und/oder Patienten: 16 konsekutive Patienten mit einem mittleren Alter von 46,3 \pm 7,7 Jahren und subfovealer Flüssigkeit aufgrund einer akuten CCS, die mit hochauflösender optischer Koherenztomographie (SD-OCT), Fluoreszein- (FA) sowie Indozyaningrünangiografie (ICGA) bestätigt wurde, wurden mit einer ICGA- gesteurten Verteporfin-PDT mit reduzierter Lichtdosis $(25 \mathrm{~J} / \mathrm{cm} 2)$ behandelt. Als Untersuchungsparameter wurden die mittlere retinale Sensitivität und die Fixationsstabilität gewählt, die mittels Mikroperimetrie (MP-1; Nidek) bestimmt wurden.

Ergebnisse: Die mittlere retinale Sensitivität verbesserte sich von 16,4 $\pm 3,0 \mathrm{~dB}$ vor der Behandlung, auf 18,2 $\pm 2,4 \mathrm{~dB}$ (p.<0.001) nach Monat 1 , auf $19.5 \pm 0.9 \mathrm{~dB}$ $(\mathrm{p}<0.001)$ nach Monat 3 und auf $19.0 \pm 1.3 \mathrm{~dB}(\mathrm{p}=0.007)$ nach Monat 6. Über den Untersuchungszeitraum war kein signifikanter Unterschied im Fixationsverhalten festzustellen. Bei allen Patienten waren 1 Monat nach der Behandlung die Quellpunkte in der FA nicht mehr nachzuweisen und das SD-OCT zeigte eine vollständige Resorption der subretinalen Flüssigkeit.

Schlussfolgerungen: Die ICGA-gesteuerte Verteporfin PDT mit reduzierter Lichtdosis eröffnet vielversprechende Perspektiven in der Therapie der akuten Form von CCS. Nach dieser Behandlung verbesserte sich die retinale Sensitivität bei gleich bleibendem Fixationsverhalten.

Schlüsselwörter: Chorioretinpathia centralis serosa, retinale Sensitivität, Fixationsstabilität, Mikroperimetrie, photodynamische Therapie.

Key words: Central serous chorioretinopathy, retinal sensitivity, fixation stability, microperimetry, photodynamic therapy.

45. P. Haas, A. Dossenbach, S. Binder (Wien): Komplementfaktor-Polymorphismen und altersbedingte Makuladegeneration (Vortrag) - Complement receptor polymorphismn and age-related macular degeneration

Problemstellung: Die Assoziation der Tyr402His-Variante von Komplementfaktor $\mathrm{H}(\mathrm{CFH})$ mit einer altersassoziierten Makuladegeneration (AMD) wurde für verschiedene kaukasische Populationen gezeigt. Die Ergebnisse für andere Polymorphismen des Komplementsystems sind teilweise widersprüchlich. Wir haben die Verteilung des Einzelnukleotid-Polymorphismen (SNP) vom Komplementrezeptor $1 \mathrm{im}$ Vergleich bei Patienten mit einer exsudativen AMD und gesunden Kontrollen untersucht.

Methode und/oder Patienten: Es wurden 50 Patienten mit exsudativer AMD und 48 Kontrollen ohne AMD eingeschlossen. Genomische DNA wurde aus Vollblut 
isoliert. Die Polymorphismen wurden mittels PCR Amplifikation und anschließender Sequenzierung des relevanten Genbereichs identifiziert.

Ergebnisse: Eine signifikante Assoziation mit exsudativer AMD wurde nur für SNP rs1061170 (Y402 H) im CFH-Gen gefunden. Der Polymorphismus im Komplementrezeptor 1 Gen zeigte hingegen nur eine positive Tendenz.

Schlussfolgerungen: Weitere Komponenten des Immunsystems, vor allem auf Chromosom 1, sind zu beachten und zu untersuchen. Unserer Studie bestätigt die große Rolle des Komplementsystems als Risikofaktor für die altersbedingte Makuladegeneration.

Schlüsselwörter: Komplement system; Chromosom 1; Altersbedingte Makuladegeneration.

Key words: Complement system; chromosom 1; age-related macular degeneration.

46. C. Kiss, M. Munk, F. Sulzbacher, S. Eisenbölbl, P. Roberts, U. Schmidt-Erfurth (Wien): Kombinierte Hemmung von Platelet Derived (PDGF) und Vascular Endothelial (VEGF) Growth Factor zur Therapie der neovaskulären AMD - Phase I Ergebnisse (Vortrag) - Targeting platelet derived (PDGF) and vascular endothelial (VEGF) growth factors for neovascular AMD - Phase 1 Study

Problemstellung: Monotherapy VEGF inhibition does not result in neovascular regression. Pericyte coverage of neovascular tissue is primarily responsible for this resistance. Blocking PDGF and VEGF thereby targeting pericytes and endothelial cells respectively may result in enhanced visual outcome. The purpose of the present study was to assess safety of E10030, an anti-PDGF pegylated aptamer combined with ranibizumab for neovascular AMD.

Methode und/oder Patienten: Predominantly or minimally classic neovascular AMD lesions were treated in a dose escalation scheme with 3 monthly E10030 injections $(0.03,0.3,1.5$, or $3.0 \mathrm{mg})$ combined with monthly ranibizumab injections. Ergebnisse: No drug related adverse events were detected. $59 \%$ of subjects gained $\geq 15$ letters at week $12(n=22)$, mean letter gain was +14.0 , mean change in OCT center point was $-157 \mu \mathrm{m}$, and the mean magnitude of neovascular regression was $86 \%$.

Schlussfolgerungen: E10030 combined with ranibizumab was well tolerated with resultant significant neovascular regression. Although the study is limited with a small number of patients and lack of a control group, it provides initial evidence of potential bioactivity associated with neovascular regression.

Schlüsselwörter: CNV, AMD, VEGF, PDGF.

Key words: CNV, AMD, VEGF, PDGF.

47. G. Seidel, A. Haas, A. Wedrich, A. Martischnig (Graz): Agent-Based Modell zur Berechnung der zu erwartenden Bevacizumabapplikationen in der Steiermark (Kurzvortrag) - Agent-based modeling for estimating the need of intravitreal Bevacizumab injections in age-realte macular degeneration in Styria

Problemstellung: Die Berechnung der zu erwartenden operativen intravitrealen Bevacizumab Applikationen bei Patienten mit altersbabhängiger Makuladegeneration (AMD) von 2010 bis 2040 in der Steiermark.

Methode und/oder Patienten: Ein Agent-Based-Modell, basierend auf Daten aus aktueller Literatur, Statistik Austria und Auswertungen unserer Patientendaten, wurde entwickelt. Die Inzidenz wurde aus der Rotterdam Studie und die Prävalenz aus der EUREYE Studie übernommen. Unseren Patientendaten entsprechend, wurde die Behandlungsmöglichkeit der AMD mi $60 \%$ bei ersten Augen und $90 \%$ bei Partneraugen angenommen. Ebenso wurde die durchschnittliche
Bemerkungen 
jährliche IVOM-Zahl mit 4,5 im ersten, 1,5 im zweiten und 1,0 im dritten Jahr angenommen. Eine kumulative Inzidenz bezüglich der Erkrankung des Partnerauges bei feuchter AMD von 40\% innerhalb von 5 Jahren wurde berücksichtigt.

Ergebnisse: Ein stetiger Anstieg von IVOMs war über den gesamten Beobachtungszeitraum festzustellen. Während im Jahr 20102320 (SD 1700-3000) Bevazizumab Applikationen errechnet wurden, waren es 20403480 (SD 2700-3400).

Schlussfolgerungen: Obwohl die Anzahl der Bevacizumab Behandlungen für die Indikation der AMD zunehmen wird, ist dieser Anstieg flacher als erwartet. Tendenzen einer Abflachung der Kurve gegen Ende der Dreißigerjahre lassen sich erahnen.

Schlüsselwörter: Agent based model, Mikromodell, IVOM, Avastin, Bevacizumab.

Key words: agent based model, Avastin, Bevacizumab.

48. T. Georgi, D. Ivastinovic, R. Hornig, M. Brandner, M. Velikay-Parel (Graz): Der Einfluss der visuellen Funktion auf die Orientierung während des Graz Mobility Tests (Kurzvortrag) - The impact of visual function on orientation during the Graz Mobility Test

Problemstellung: In vorangegangenen Studien wurde der Graz Mobility Test (GMT) vorgestellt und die Beeinflussung der Resultate bei wiederholten Testungen durch Lernverhalten untersucht. Da die zu erwartende Verbesserung bei Artificial Vision sehr niedrige Visusstufen betrifft, muss davon ausgegangen werden, dass bei der Verbesserung der Performance auch das Gesichtsfeld maßgeblich beteiligt ist. Der GMT als funktioneller Test untersucht die Kombination beider wichtigen Sehfunktionen und gibt einen praxisrelevanten Aufschluss für das täglichen Leben. In dieser Studie wird der Zusammenhang von visueller Funktion (Sehschärfe und Gesichtsfeld) und der Orientierung während des Graz Mobility Test dargestellt.

Methode und/oder Patienten: Low-Vision Patienten $(n=16)$ mit Sehschärfen zwischen Lichtempfinden und 1,0 logMAR absolvierten einen Mobilitäts-Test, welcher aus vier verschiedenen, strukturell ähnlichen Labyrinthen mit schwarzen Hindernissen besteht. Jeder Test wurde vier- bis sechsmal absolviert. Mithilfe von 8 Kameras wurden horizontale und vertikale Blickbewegungen des Kopfes während des Testdurchgangs aufgezeichnet. Die Anzahl und Winkel der Kopfbewegungen konnten durch ein speziell entwickeltes Computerprogramm aufgezeichnet und ausgewertet werden. Gesichtsfeld, Anzahl der Kontakte, Durchgangszeit, Frequenz der Kopfbewegungen und Durchschnittswinkel waren die Parameter.

Ergebnisse: Die Probanden wurden in zwei Gruppen entsprechend ihrem Visus und Gesichtsfeld eingeteilt. Die niedrigere Visusgruppe hatte eine höhere Durchgangszeit, mehr Kontakte und mehr Scanningbewegungen des Kopfes. Der Unterschied zwischen den Gruppen war in allen Parametern signifikant. Der Durchschnittswinkel unterschied sich nicht signifikant. Subgruppenanalysen zeigten, dass das Gesichtfeld einen wesentlichen Einfluss auf die Anzahl der Kontakte und auf die Frequenz der Kopfbewegungen hatte. Patienten mit exzentrischem Gesichtfeld zeigten deutlich weniger Scanningbewegungen. Es waren jedoch große individuelle Varianzen in allen Gruppen zu beobachten.

Schlussfolgerungen: Mit Hilfe des GMT können Unterschiede der visuellen Funktion signifikant dargestellt werden. Die zu erwartenden Visusverbesserungen im Rahmen des Artificial Vision können somit aufgezeigt werden. Durchgangszeit, Anzahl der Kontakte und die Scanningbewegungen des Kopfes sind jeweils für sich relevante Messgrößen.

Schlüsselwörter: Künstliches Sehen, Orientierung, niedrige Visusstufen.

Key words: Artificial vision, orientation, low-vision. 
49. J. Kroisamer, R. Sayegh, C. Simader, U. Scheschy, A. Montuoro, I. Womastek U. Schmidt-Erfurth (Wien): Charakteristika des Fixationsverhaltens von Patienten mit Geographischer Atrophie gemessen mit dem MP-1 Mikroperimeter (Vortrag) - Characteristics of fixation in patients with geographic atrophy recorded with the MP-1 microperimeter

Problemstellung: Ziel dieser Studie war es, das Fixationsverhalten von Patienten mit geographischer Atrophie mit Hilfe des MP-1 Mikroperimeters zu analysieren.

Methode und/oder Patienten: Mit Hilfe des MP-1 Mikroperimeters (Nidek Technologies, Softwareversion 1.4.2) wurde eine statische Schwellenwertperimetrie inklusive Fixationsanalyse bei 58 Augen mit geographischer Atrophie durchgeführt. Die Fixationsstabilität wurde in 3 Kategorien eingeteilt: • „Stabil“ • „Relativ Unstabil“ • „Unstabil“ Um die korrekte Position der Fovea zu bestimmen wurde die foveale Depression am Spectralis OCT Bild des Patienten markiert. Anschließend wurde das entsprechende SLO Bild mit dem IR Fundusbild des MP-1 überlagert. Abhängig von der Anzahl jener Fixationsmessungen welche innerhalb eines Kreises mit 2 Durchmesser um die Fovea lagen erfolgte eine Einteilung der Fixation in: • „Überwiegend Zentral“ • „Selten Zentral“ • „Überwiegend Exzentrisch“ Bei Patienten mit überwiegend exzentrischer Fixation wurde die Position des neu etablierten Fixationsbereichs bestimmt.

Ergebnisse: Die Patienten dieser Studie zeigten folgende Fixationsmerkmale: • „Überwiegend Zentral“ (6,9\%): „Stabil“ (100\%), „Relativ Unstabil“ (0\%), „Unstabil“ (0\%) • „Selten Zentral“ (1,72\%): „Stabil“ (0\%), „Relativ Unstabil“ (100\%), „Unstabil“ (0\%) • „Überwiegend Exzentrisch“ (91,38\%): „Stabil“ (9,43\%), „Relativ Unstabil“ (37,74\%), „Unstabil“ (52,83\%) Der neu etablierte Fixationsbereich lag im Fundusbild superior links $(43,1 \%)$, superior rechts $(17,24 \%)$, links $(15,52 \%)$, superior $(13,79 \%)$ oder inferior links $(1,72 \%)$ der Atrophie. Kein Patient zeigte einen Fixationsort in einem der drei übrigen Segmente.

Schlussfolgerungen: Patienten mit geographischer Atrophie zeigen eine Tendenz zu exzentrischer Fixation mit einem neuen Fixationsbereich superior bzw. links der Atrophie. Diese Ergebnisse stimmen gut mit früheren Untersuchungen überein. Deshalb kann Mikroperimetrie mit dem MP-1 als eine geeignete Methode zur Evaluierung des Fixationsverhaltens angesehen werden.

Schlüsselwörter: Geographische Atrophie, Fixationsverhalten, Mikroperimetrie. Key words: Geographic atrophy, fixation pattern, microperimetry.

50. M. Georgopoulos, S. Sacu, M. Bolz, K. Eibenberger, G. Weigert, G. Mylonas, U. Schmidt-Erfurth (Wien): Untersuchung morphologischer Charakteristika der Makula-off-Netzhautabhebung mittels OCT (Vortrag) - Characteristic morphology of detached macula at rhegmatogenous retinal detachment evaluated with OCT

Problemstellung: Die Abhebung der zentralen Netzhaut („Makula-off“) gilt für den postoperativ maximal erreichbaren Visus als prognostisch ungünstiges Kriterium. In dieser retrospektiven Untersuchung wird die Morphologie der abgehobenen Netzhaut bei Patienten mit Makula-off Netzhautabhebung mit der optischen Kohärenztomographie (OCT) untersucht. Unterschiedliche morphologische Formen werden definiert und ein Bezug zur zeitlichen Komponente hergestellt. Die unterschiedlichen postoperativen OCT- und Visusergebnisse werden dem gegenüber gestellt.

Methode und/oder Patienten: Bei Patienten mit Makula-off Netzhautabhebung werden präoperativ außer Snellen-Visus, Spaltlampen- und Fundusuntersuchung (mit Fundusschema-Zeichnung) auch Schnittbilder der abgehobenen Netzhaut mittels OCT angefertigt (hochauflösende Scans und 3D-Bilder). Verschiedene morphologische Formen der abgehobenen Netzhaut im Makulabereich werden im OCT erfasst. Postoperativ werden maximal erreichter Visus und 
Netzhautmorphologie im OCT Bild untersucht. Auch die verwendete Operationsmethode (Buckelchirurgie oder Vitrektomie) wird in die Analyse mit einbezogen.

Ergebnisse: Von den 189 konsekutiv erfassten primären Netzhautabhebungen präsentierten sich 117 Patienten mit einer abgehobenen Makula (62\%). Bei 55 (47\%) dieser Makula-off Netzhautabhebungen war es möglich, präoperativ eine OCT-Untersuchung durchzuführen. Die 3D-OCT-Bilder trugen zu einer besseren Beurteilung der zentralen Abhebung bei. Die morphologische Untersuchung der abgehobenen Netzhaut ergab folgende typische Muster: intraretinale Zystenbildung, wellenförmige Veränderungen der äußeren Netzhautschichten, äußere Verdichtungszonen und Kombinationen. Eine chronologische Abfolge der präoperativen Formen in Abhängigkeit von Dauer und Höhe der Makula-off Abhebung, sowie postoperative morphologische und funktionelle Ergebnisse werden präsentiert.

Schlussfolgerungen: Makula-off Netzhautabhebungen sollten differenziert betrachtet werden. Es können verschiedene Haupttypen der Veränderung der Netzhaut bei abgehobener Makula im OCT und ihre Korrelation mit dem endgültigen Visus definiert werden. Das 3D-OCT ist eine wertvolle Ergänzung einerseits zur Beurteilung der präoperativen Situation als auch des postoperativen Verlaufs.

Schlüsselwörter: Netzhautablösung, Makula off, OCT, Morphologie, Netzhautschichten.

Key words: Ablatio retinae, rhegmatogenous retinal detachment, macula off, OCT, morphology, retinal layers.

51. C. Mitsch, M. Bolz, C. Scholda, U. Schmidt-Erfurth (Wien): Morphologische Analyse von peripheren retinalen Ischämiearealen bei Patienten mit diabetischer Retinopathie. (Vortrag) - In-vivo morphologic analysis and follow-up of peripheral retinal ischemia secondary to diabetic retinopathy

Problemstellung: Periphere morphologische Veränderungen bei diabetischer Retinopathie: Analyse von morphologischen Veränderungen in peripheren retinalen Ischämiearealen, die mittels Fluoreszenz-Angiographie (FA) lokalisiert und mit der spektral domain optischen Kohärenztomographie (SD-OCT) aufgenommen wurden.

Methode und/oder Patienten: Die in eine Studie zur Therapie des diabetischen Makulaödems inkludierten PatientInnen wurden mit dem Heidelberg Engineering ${ }^{\circledR}$ Spectralis HRA+OCT ${ }^{\mathrm{TM}}$ in der FA und mit SD-OCT im Jahresintervall untersucht. Periphere Ischämieareale wurden lokalisiert und zumindest ein hochauflösender SD-OCT Line-Scan eines Ischämieareals aufgenommen. Die Aufnahmen wurden verglichen und hinsichtlich Durchmesser, Umriss und Morphologie untersucht.

Ergebnisse: Periphere Ischämieareale bei Patienten mit diabetischer Retinopathie ließen sich aufgrund ihrer Morphologie eindeutig in zwei Gruppen unterteilen: Homogene, diskret hyperreflektive Bänder bzw. stärker hyperreflektive, heterogen reflektive Läsionen, die einen cotton-whool-spot darstellen und sich zwischen Ganglionzellschicht und Nervenfaserschicht erstrecken. Bei allen Patienten nahm die Dicke der Läsionen in den SD-OCT-Aufnahmen im Verlauf eines Jahres ab. Der Durchmesser der horizontalen Bänder in den SD-OCT-Aufnahmen korrelierte mit dem Durchmesser der ischämischen Areale auf der FA. Das Pigmentepithel und die Photorezeptor-Schicht unterhalb dieser Läsionen zeigten eine normale Morphologie: die Außen- und Innensegmente, das Pigmentepithel und die äußere nukleäre Schicht zeigten keine Ischämie bedingten Veränderungen.

Schlussfolgerungen: Ischämieareale lassen sich in der SD-OCT als eindeutige pathomorphologische Entität identfizieren. Größe und Umriss können in der SD-OCT und in der FA mit vergleichbarer Eindeutigkeit erkannt werden. Der 
pathologische ischämische Prozess scheint auf die Ganglienzellschicht und die Nervenfaserschicht limitiert zu sein. Die retinale Morphologie unterhalb dieser Schichten zeigen keine signifikante Veränderung.

Schlüsselwörter: Optische Kohärenztomographie, Periphere Ischämieareale, Diabetische Retinopathie, Fluoreszenzangiographie.

Key words: Optical coherence tomography, peripheral retinal ischemia, diabetic retinopathy, fluorescein angiography.

16:13

52. A. Prinz, B. Weingessel, P. Irsigler, P. Vécsei-Marlovits (Wien): Morphologische und funktionelle Veränderungen nach Makulachirugie bei epiretinaler Fibrose (Vortrag) - Morphological and functional changes after epiretinal membrane surgery.

Problemstellung: Ziel der vorliegenden Studie war es zu klären, ob die mittels hochauflösender optischer Kohärenztomographie (OCT) erfassten morphologischen Veränderungen mit den funktionellen Veränderungen im zeitlichen Verlauf korrelieren.

Methode und/oder Patienten: In dieser retrospektiven Studie wurden alle makulachirurgischen Eingriffe an PatientInnen mit epiretinaler Fibrose (ERF), die im KH Hietzing kontinuierlich von Jänner bis Juni 2010 durchgeführt wurden, hinsichtlich postoperativen Veränderungen in Nah- und Fernvisus, OCT und subjektiven Sehbefinden analysiert. Alle Eingriffe, gegebenenfalls mit KataraktOperation kombiniert, wurden mittels 23 gauge pars plana Vitrektomie, Färbung mit Brilliant blue, Membranpeeling und Delamination der Membrana limitans interna (ILM) durchgeführt. Nahvisus, Fernvisus, subjektive Metamorphopsien und zentrale Netzhautdicke im OCT wurden präoperativ, 1 Woche, 1 Monat und 6 Monaten postoperativ verglichen.

Ergebnisse: Bei den insgesamt 20 Augen von 18 PatientInnen zeigten sich 6 Monate postoperativ sowohl ein signifikanter Fernvisusanstieg von $0,3 \pm 0,2$ auf $0,6 \pm 0,2$ Snellen (p.<0.001) als auch ein Nahvisusanstieg von 4,8 $\pm 3,4$ auf 2,7 $\pm 3,1$ Jägertafeln Textgrössen ( $\mathrm{p}<0,01$ ). Die zentrale Netzhautdicke nahm nach 6 Monaten von 416,8 $\pm 69,4 \mu \mathrm{m}$ auf $361 \pm 68,3 \mu \mathrm{m}$ signifikant ab $(\mathrm{p}=0,01)$ Im zeitlichen Verlauf kam es zu einem signifikanten Fernvisusanstieg zu jedem Kontrollzeitpunkt. Zwischen der 1 Woche- und 1 Monatskontrolle kam es zu einer signifikanten Nahvisusbesserung $(p=0,3)$. Die Morphologie der IS/OS Junction im OCT korrelierte sowohl präoperativ als auch postoperativ mit dem Fernvisus $(p=0,01)$ und präoperativ mit der Netzhautdicke $(p=0,04)$.

Schlussfolgerungen: Der postoperative Verlauf wird signifikant von dem präoperativen Visus und den IS/OS-Junction Veränderungen im OCT beeinflusst. Die signifikanten funktionellen Veränderungen hinsichtlich Visusverlauf treten kontinuierlich auf und gehen den im OCT nachgewiesenen morphologischen Veränderungen voraus.

Schlüsselwörter: Hochauflösendes OCT, Makulachirurgie, Epiretinale Fibrose, IS/OS Junction.

Key words: High resolution OCT, epiretinal membrane surgery, IS/OS junction.

$16: 22$

53. C. Simader, G. Deak, M. Bolz, G. Mylonas, S. Prager, B. Hajnajeeb, U. Schmidt-Erfurth (Wien): Qualitätsoptimierung für optische Kohärenztomographie aus Sicht einer Klinikambulanz und eines Readingcenters (Vortrag) - How to receive high-quality optical coherence tomography results: the perspective of an outpatient clinic and a reading center

Problemstellung: Die Optische Kohärenztomographie (OCT) hat als nichtinvasive und schnell durchzuführende Untersuchungstechnik die Diagnostik an Zentren mit Schwerpunkt Netzhauterkrankungen revolutioniert und findet nun
Bemerkungen 
auch immer mehr Einzug in den niedergelassenen nicht spezialisierten Bereich. Gab es bei der time-domain basierten Technologie (TD-OCT) nur ein Gerät, sind heute mit der spectral-domain (SD-OCT) basierten Technologie inzwischen zahlreiche Geräte verschiedener Hersteller mit differierenden Aufnahmeprotokollen und Zusatzfunktionen auf dem Markt. Dies erfordert sowohl für die Diagnostik im Rahmen des Erstbesuchs als auch für die Verlaufskontrolle exakt definierte Standards.

Methode und/oder Patienten: Im Rahmen der Ambulanz für Innere Augenerkrankungen der Universitätsklinik für Augenheilkunde und Optometrie in Wien werden täglich 40 bis 50 Patienten untersucht. In $20 \%$ der Fälle wird durch eine(n) eingeschulte(n) Orthoptisten(in) eine SD-OCT Untersuchung mit einem Topcon 3D OCT-1000 zur Unterstützung der Diagnostik eingesetzt. Im Vienna Readingcenter (VRC) der Universitätsklink für Augenheilkunde in Wien, einem Auswertungszentrum internationaler multizentrischer Studien werden monatlich über 1000 OCT Untersuchungen, aufgenommen von vorher zertifizierten Untersuchern, ausgewertet. SD-OCT Aufnahmen der Ambulanz und des VRC wurden retrospektiv bezüglich Qualität und Ursache des eventuell vorliegenden Qualitätsverlusts untersucht.

Ergebnisse: Der häufigste Grund eines Qualitätsverlusts der in der Ambulanz durchgeführten Untersuchungen war verringerter Bildkontrast. In einem Großteil dieser Fälle wurde eine zweite Aufnahme mit einem Cirrus SD OCT (Zeiss Meditec) durchgeführt. Gelegentlich konnte durch leicht verbessertem Kontrast die Therapieentscheidung erleichtert werden. Im Falle von Bewegungsartefakten während der Aufnahme war ein Wechsel auf das trackingsystembasierte Spectralis OCT (Heidelberg Engineering) unter Verwendung einer niedrigen Framezahl manchmal erfolgreich. Schräge Scans wurden sehr selten, abgeschnittene Scanbereiche wurden nie gesehen. Auswertungen von im Readincenter eingelangten Untersuchungen ergaben deutlich häufiger schräge, aber auch ageschnittene Scans. Untersucher, die ihre Zertifizierung bereits im ersten Durchgang erfolgreich absolvierten, lieferten seltener Scans mit Qualitätsverlust.

Schlussfolgerungen: Alle in der Ambulanz und im VRC akzeptierten Geräte zeigen gute Qualitätsergebnisse. In fast allen Ambulanzfällen kann der geringe Kontrast durch Medientrübungen erklärt werden. Ein Zertifizierungsprozess, wie für die Teilnahme an multizentrischen Studien vom VRC gefordert, hat sich bewährt. Eine Adaptierung dieser Zertifizierung für den klinischen Alltag wurde vorgenommen. Sie dient ab nun als Voraussetzung für alle Untersucher der Augenklinik in Wien zur Durchführung für SD-OCT Aufnahmen. Zum Zwecke weiterer Qualitätsverbesserung scheint jedoch auch ein regelmäßiges Azt-Untersucher Feedback notwendig.

Schlüsselwörter: OCT, Qualität, Readingcenter.

Key words: OCT, quality, reading center.

$16: 31$

54. F. Schlanitz, M. Bolz, B. Baumann, M. Platzer, M. Pircher, C. Hitzenberger, U. Schmidt-Erfurth (Wien): Charakteristiken von Drusen in der Polarisationssensitiven Optischen Kohärenztomographie (Vortrag) - Drusen characteristics imaged with polarization-sensitive optical coherence tomography

Problemstellung: Ziel der Studie war es, die verschiedenartige Struktur von Drusen der altersbedingten Makuladegeneration mithilfe der Polarisationssensitiven Optischen Kohärenztomographie (PS-OCT) zu beschreiben und zu klassifizieren.

Methode und/oder Patienten: 15 Augen von 15 Patienten mit Drusen der Klassifikation AREDS 2/3 wurden mit dem PS-OCT untersucht. Die Studie entsprach den Bestimmungen der Deklaration von Helsinki. Jeder einzelne B-scan wurde von zwei voneinander unabhängigen Experten untersucht. Die Position, Größe, Geometrie und spezielle Charakteristik jeder einzelnen Druse wurde evaluiert und in einer „Drusenkarte“ der Netzhaut dargestellt. Zudem wurde mithilfe der 
polarisationssensitiven Information der Zustand des der Druse überliegenden retinalen Pigmentepithels (RPE) festgestellt, wie auch das eventuelle Vorhandensein pigmentepithelialer Verschiebungen.

Ergebnisse: Signifikant abhängig von ihrer Größe $(p<0,01)$.

Schlussfolgerungen: Die Polarisationssensitive Optische Kohärenztomographie erlaubt eine qualitative Beurteilung von Drusen und deren überliegenden RPE sowie pigmentepithelialer Verschiebungen. Die verschiedenen Beziehungen zwischen Drusenform und -größe und der Integrität des überliegenden RPEs dürften sowohl für die Grundlagenforschung als auch klinische Praxis der frühen altersbedingten Makuladegeneration von Nutzen sein.

Schlüsselwörter: Drusen, OCT, RPE.

Key words: Drusen, OCT, RPE.

55. S. Prager, K. Kriechbaum, B. Gerendas, G. Mylonas, M. Bolz, G. Rainer, U. Schmidt-Erfurth (Wien): Morphologische Charakteristika im SD OCT, die mit einer Visus Besserung nach Injektionstherapie bei diabetischem Makulaödem assoziiert werden können. (Vortrag) - Morphological factors in SD OCT associated with improvement of visual acuity after intravitreal injections in diabetic macular edema

Problemstellung: Intravitreale Applikation von Triamcinolon oder anti VEGF zeigt sich in verschiedenen Studien als effektive Alternative zur Laserkoagulation bei diabetischem Makulaödem. Unklar ist, warum manche Patienten von einer Therapie profitieren, andere nicht. In dieser prospektiven Studie wurden Pathomorphologien im SD OCT mit der Visus-Änderung nach drei Monaten Therapie korreliert.

Methode und/oder Patienten: 32 Patienten mit zuvor unbehandeltem, zentralem diabetischem Makulaödem wurden in diese prospektive verblindete Studie inkludiert. Sie wurden in zwei gleiche Gruppen randomisiert: 16 Patienten erhielten drei intravitreale Bevacizumab Injektionen (2,5 mg) im monatlichen Abstand, 16 Teilnehmer erhielten zu Beginn eine intravitreale Triamcinolon Injektion ( $8 \mathrm{mg}$ ) gefolgt von zwei Scheininjektionen. Von allen Patienten wurden vor Therapiebeginn SD-OCT Bilder (Cirrus, Zeiss) aufgenommen und hinsichtlich folgender Kriterien ausgewertet: Vorkommen von subretinaler Flüssigkeit (SRF), zentrale Netzhautdicke, Größe der intraretinalen Zysten und Integrität der Photorezeptorschicht.

Ergebnisse: Die Studienpopulation ließ sich abhängig von der Visusänderung 3 Monate nach Therapiebeginn in zwei Gruppen unterteilen. 23 Patienten zeigten eine stabile oder verbesserte Sehleistung mit einem durchschnittlichen VisusAnstieg von 6 Buchstaben, 9 Patienten hatten einen durchschnittlichen VisusVerlust von 6 Buchstaben. Beide Gruppen waren bezüglich Alter, Geschlecht, Ausgangs-Visus und zentraler Netzhautdicke vergleichbar. In beiden Behandlungsgruppen waren eine intakte Photorezeptorschicht sowie das Vorkommen von subretinaler Flüssigkeit vor Behandlungsbeginn mit einem Visus-Anstieg nach drei Monaten Therapie assoziiert. Weiters zeigten Patienten der Bevacizumab Gruppe nach 3 Monaten eine bessere Sehleistung im Vergleich zur Triamcinolon Gruppe, obwohl der Rückgang an zentraler Netzhautdicke in beiden Gruppen vergleichbar war.

Schlussfolgerungen: Die Ergebnisse dieser Studie lassen vermuten, dass sich subretinale Flüssigkeit und eine intakte Photorezeptorschicht prognostisch positiv auf eine funktionelle Besserung nach Injektionstherapie bei diabetischem Makulaödem auswirken.

Schlüsselwörter: Diabetisches Makulaödem, OCT, Bevacizumab, Triamcinolon. Key words: Diabetic macular edema, OCT, bevacizumab, triamcinolone. 


\section{NETZHAUT 2}

Vorsitz: S. Egger, A. Wedrich

56. C. Falkner-Radler, S. Binder (Wien): Primäre Pars-plana-Vitrektomie-360 Endolaserphotokoagulation versus Cerclage zur Behandlung einer rhegmatogenen Netzhautablösung. (VEE Studie) (Vortrag) - Primary pars plana vitrectomy-360 endolaser therpay or encircling band for rhegmatogenous retinal detachment. (VEE Study)

Problemstellung: Bei der Behandlung von rhegmatogenen Netzhautablösungen konnte sich die Pars-plana Vitrektomie als vielversprechende Operationsmethode etablieren, wobei eine geringgradig bessere Heilungsrate bei zusätzlicher Cerclage beschrieben worden ist. In einer randomisierten klinischen Studie wurde die funktionelle und anatomische Heilungsrate bei der Behandlung von Patienten mit der Diagnose mittelschwere rhegmatogene Netzhautabhebung untersucht.

Methode und/oder Patienten: Insgesamt wurden 60 Patienten inkludiert, die nach dem Zufallsprinzip mit einem von zwei Operationsverfahren behandelt worden sind. Gruppe 1 wurde mit einer Pars-plana Vitrektomie und einer zusätzlichen 360 Endolasertherapie behandelt und Gruppe 2 mit einer Pars-plana Vitrektomie und einer zusätzlich Cerclage. Die intraokuläre Tamponade (Gas oder Silikonöl) wurde nach dem Schweregrad der Netzhautablösung gewählt. Hauptzielparameter waren neben der Reablatiorate, intra- und postoperative Komplikationen, postoperatives Schmerzempfinden, Refraktion und Sehvermögen.

Ergebnisse: Erste Ergebnisse zeigen eine Reablatiorate von 7\% (Je 2 Patienten pro Gruppe). In keiner von beiden Gruppen kam es zu intraoperativen Komplikationen. Die postoperativen Komplikationen inkludierten passagere Augendrucksteigerung, Iris capture und transiente Kerathopathien, und traten in beiden Gruppen in vergleichbarer Häufigkeit auf. Im Vergleich zu Gruppe 2 hatten Patienten in Gruppe 1 seltener und weniger stark ausgeprägte postoperative Schmerzen sowie eine stabilere Refraktion ( $-0,3 \pm 0,9$ Dioptrien versus $-0,9 \pm 1,0$ Dioptrien). Eine signifikante Verbesserung des Sehvermögens um 3 oder mehr Zeilen zeigte sich in beiden Gruppen in mehr als 2/3 der Patienten.

Schlussfolgerungen: Die Pars-plana Vitrektomie in Kombination mit einer 360 Endolasertherapie oder mit einer Cerclage liefert vergleichbare und vielversprechende anatomische und funktionelle Erfolgsraten bei Patienten mit der Diagnose rhegmatogene Netzhautabhebung. Mögliche Vorteile der Kombination mit dem Endolaser könnten in einem reduzierten postoperativen Schmerzempfinden und einer stabileren Refraktion liegen.

Schlüsselwörter: Rhegmatogene Netzhautabhebung, Pars-plana Vitrektomie, Endolaser Cerclage.

Key words: Rhegmatogenous retinal detachment, pars plana vitrectomy, endolaser, encircling band.

57. C. Kernstock, K. Štingl, U. Greppmaier, S. Danz, H. Sachs, K. Bartz-Schmidt, E. Zrenner (Tübingen): Elektronische subretinale Implantate der 2. Generation bei Retinitis Pigmentosa (Vortrag) - Electronic subretinal 2nd generation implants in Retinitis Pigmentosa

Problemstellung: Retinitis Pigmentosa (RP) führt zu einer weitgehenden Degeneration der retinalen Photorezeptorschicht und damit meist zu einem kompletten Verlust brauchbarer Sehfähigkeit. Neurone der inneren Netzhautschichten sind jedoch in der Regel auch bei fortgeschrittener Krankheit erhalten. Die wichtigsten Ergebnisse einer Pilotstudie, bei der elektronische subretinale Implantate die Funktion der Photorezeptoren ersetzen, wurden kürzlich in Proc. 
R.Soc.B. veröffentlicht (doi: 10.1098/rspb.2010.1747). In der derzeitigen multizentrischen Folgestudie wurden bisher 5 Patienten mit der 2. Generation eines subretinalen Implantats (Alpha IMS, Retina Implant AG, Reutlingen, Deutschland) versorgt.

Methode und/oder Patienten: Der subretinale Chip mit 1500 „pixel“ auf einer Fläche von 3 mal $3 \mathrm{~mm}$ nutzt das noch vorhandene neuronale Netzwerk der inneren Netzhautschichten für die Signalverarbeitung und Weiterleitung der Impulse bis zur Sehrinde. Die Stromversorgung des Implantats wird nicht mehr durch ein direkt verbundendes Kabel, sondern elektromagnetisch durch eine retroauriculäre, subdermale Induktionsspule sichergestellt, die mittels eines ausschließlich subdermal laufenden Kabels mit dem Chip verbunden ist. Angesteuert wird der Chip durch eine auf der Haut magnetisch haftende Primärspule hinter dem Ohr, die mit einem Stromversorgungsteil in der Jackentasche verbunden ist. Alle Patienten sind an Retinitis Pigmentosa erkrankt und hatten keine im Alltag nutzbare Sehfähigkeit (keine Lichtscheinprojektion, maximal Lichtscheinwahrnehmung).

Ergebnisse: Durch das komplett subdermal liegende Gesamtsystem ist eine gefahrlose Benutzung des Chips auch in Alltagssituationen oder zuhause möglich. Die schlaufenförmige oder cerclage-artige intraorbitale Kabelführung erlaubt normale Augenbewegungen. Die Patienten können Helligkeit und Bildkontrast selbst einstellen. Die Ergebnisse der neuen Stromversorgungsanordnung und die erzielten Sehleistungen bei aktiviertem Chip wurden denen bei deaktiviertem Chip zum Vergleich gegenübergestellt. Die Sehleistungen mit dem Chip waren interindividuell unterschiedlich und beinhalten das Erkennen von Gegenständen des Alltags, wie Besteck, Teller, Bierglas; Erkennen von Gesichtern, der Konturen der Nase sowie des Schattens der Augen, Bewegungsrichtungen von Menschen, Tieren und Autos auf der Strasse. Ein Visus von ca. 20/1000 mit Landoltringen kann erreicht werden.

Schlussfolgerungen: Elektronische subretinale Implantate ermöglichen RP-Patienten mit komplettem Verlust der Sehfähigkeit nicht nur basale Helligkeitswahrnehmungen, sondern Sehleistungen mit einer Auflösung, die eine deutliche Hilfestellung im Alltag bedeutet. Subretinale Implantate stellen zur Zeit das - experimentelle - Therapieverfahren bei Retinitis Pigmentosa dar, das die beste Wiederherstellung von Sehleistungen liefert. Die derzeitige Hauptstudie zum subretinalen Chip Alpha IMS der Retina Implant AG soll die Sicherheit und Wirksamkeit dieser Technologie in einer Langzeitbeobachtung prüfen und damit einen Beitrag zur breiteren Verfügbarkeit dieses Verfahrens leisten.

Schlüsselwörter: Subretinale Implantate, Retinitis Pigmentosa, künstliches Sehen.

Key words: Subretinal implants, Retinitis Pigmentosa, artificial vision.

58. S. Egger, N. Lang, C. Ortner, C. Nischler, D. Paikl, J. Spaun, G. Grabner (Salzburg): Epimakuläre Brachytherapie - erste Erfahrungen in der Verwendung dieser Methode zur Therapie choroidaler Neovaskularisationen (Kurzvortrag) - Epimacular brachytherapy - first experiences with this new method in the treatment of choroidal neovascularisations

Problemstellung: Die epimakuläre Brachytherapie stellt eine neue Methode zur Behandlung choroidaler Neovaskularisationen (CNV) dar. Durch lokalisierte Bestrahlung der Läsion mit dem Betastrahler Strontium 90 soll eine Inhibition der Proliferation der CNV erreicht werden. Über erste Erfahrungen in der klinischen Anwendung dieser Methode wird berichtet.

Methode und/oder Patienten: Nach ausführlicher Aufklärung wird bei den Patienten eine 23g pars plana Vitrektomie mit Entfernung der zentralen Glaskörperanteile durchgeführt. Im Anschluss daran erfolgt die lokalisierte Bestrahlung der CNV mit Hilfe eines intraocular zu verwendenden Strontium 90 Applikator Gerätes (Vidion System). Es wird eine Dosis von 24 Gray appliziert, die Bestrahlungsdauer liegt bei etwa 4 Minuten. Am Ende der Operation wird einmalig eine Anti-VEGF Substanz (Ranibizumab) intravitreal verabreicht. 
Ergebnisse: Über die bisher vorliegenden Ergebnisse internationaler multizentrischer Studien sowie die Erfahrungen mit den bisher in Salzburg behandelten Patienten wird berichtet.

Schlussfolgerungen: Die epimakuläre Brachytherapie stellt möglicherweise eine vielversprechende Methode zur Therapie choroidaler Neovaskularisationen dar. Da Langzeitergebnisse zum gegenwärtigen Zeitpunkt aber noch ausständig sind, wird diese Methode in Salzburg nur im Rahmen von Studien angewendet.

Schlüsselwörter: Epimakulär, Brachytherapie, choroidal, Neovaskularisation, altersbedingt, Makuladegeneration.

Key words: Epimacular, brachytherapy, choroidal, neovascularisationi, age, related, macular, degeneration.

59. J. Schmidt, S. Mennel (Duisburg): Perioperative Anti- VEGF Applikation bei der Pars- plana Vitrektomie (PPV) des diabetischen Auges (Vortrag) Perioperative anti-VEGF administration in the pars plana vitrectomy (PPV) of the diabetic eye

Problemstellung: Bei Diabetikern mit progredienter proliferativer Retinopathie und persistierendem Makulaödem ist eine Pars- plana Vitrektomie erforderlich.

Methode und/oder Patienten: Die diabetischen Augen, die einer PPV bedürfen, weisen häufig massive proliferative Veränderungen sowohl der Netzhaut als auch der Iris auf. Intraoperativ und im postoperativen Verlauf kommt es hier häufiger zu schwer zu beherschenden Nachblutungen.

Ergebnisse: Die intravitreale Applikation von Anti-VEGF einige Tage vor der PPV reduziert die Hyperämie der retinalen Vasoproliferationen und der Iris, so dass sich intraoperativ die präretinalen Membranen leichter en-bloc exzidieren lassen und durch die geringe Blutngsneigung weitgehend auf Koagulationen verzichtet werden kann. Eine Luftendotamponade reduziert weiter die postoperative Nachblutung.

Schlussfolgerungen: Bei Patienten mit gestörter Iristrophik und Schrankenstörungen wie bei Diabetikern gestaltet sich durch die Anwendung von Anti- VEGF das operative Vorgehen deutlich übersichtlicher. Auf eine hämostypische Endotamponade mit Silikonöl kann meist verzichtet werden und ein Makulaödem wird mit behandelt.

Schlüsselwörter: Anti-VEGF, Pars- plana Vitrektomie (PPV), PDVR.

Key words: Anti-VEGF, pars- plana vitrectomy (PPV), PDVR.

60. C. Scholda, M. Bolz, U. Schmidt-Erfurth (Wien): Die Bedeutung der UltraWeitwinkel-Fluoreszenz-Angiografie (Optomap ${ }^{\circledR}$, Optos PLC ${ }^{\circledR}$, UK) für Diagnostik und Therapie der Diabetischen Retinopathie (Vortrag) - The value of ultra-wide field fluorescence angiografy (Optomap ${ }^{\circledR}$, Optos PLC ${ }^{\circledR}$, UK) for diagnosis and therapy of diabetic retinopathy

Problemstellung: Die diabetische Retino- und Makulapathie sind Folge einer generalisierten Alteration des retinalen Gefäßsystems. Die Fluoreszenzangiografie stellt neben der OCT eine der wichtigsten diagnostischen Methoden in der Diagnostik und Verlaufskontrolle der diabetischen Netzhauterkrankungen dar. Mit konventionellen FLA-Geräten kann die angiografische Darstellung der Netzhautperipherie nur sehr eingeschränkt erfolgen. Ein neues Ultraweitwinkel-Angiografie-Gerät (Optomap ${ }^{\circledR}$, Optos PLC $^{\circledR}$ ) ermöglicht die Erfassung der zentralen und peripheren Netzhautpathologie.

Methode und/oder Patienten: Das auf Scanning-Laser-Ophthalmoskop-(SLO)Technologie basierende Gerät erlaubt durch den Einsatz einer speziellen Spiegeloptik bis zu 200 Grad der gesamten und auch peripheren Netzhaut abzubilden. In Kombination mit einer FLA-Einrichtung kann so die fluoreszenzangiografi- 
sche Darstellung der Netzhautperipherie mit Identifikation ischaemischer Areale im Rahmen der DR erfolgen.

Ergebnisse: Die Darstellung der gesamten Netzhaut in ihren zentralen und peripheren Anteilen mittels Ultra-Weitwinkel-FLA erlaubt einen bisher unerreichten Einblick in die Pathogenese sowohl des diabetischen Maculaödems als auch der prä-/proliferativen diabetischen Retinopathie. Die Identifikation von ischaemischen Arealen und Leckagen in der peripheren Netzhaut ermöglicht eine spezifischere Therapie, sowie eine bessere Verlaufskontrolle und effizientere Therapie. Insbesondere mit Rücksicht auf die neuen antiangiogenetischen Therapiestrategien beim diabetischen Makulaoedem ist eine umfassende Diagnostik der ischämischen Gesamtsituation von wesentlicher Bedeutung.

Schlussfolgerungen: Die neue Ultraweitwinkel-Fluoreszenz-Angiografie stellt eine wesentliche Innovation in der Diagnostik und Therapie der diabetischen Retinopathie dar. Die damit mögliche angiografische Erfassung der Netzhautperipherie könnte zu einer Modifikation der therapeutischen Strategien im Rahmen der diabetischen Retinopathie führen.

Schlüsselwörter: Diabetische Retinopathie, Fluoreszenzangiografie, proliferative diabetische Retinopathie, diabetisches Maculaödem, antiangiogenetische Therapie.

Key words: Diabetic retinopathy, fluorescein angiografy, proliferative diabetic retinopathy, diabetic macular edema, antiangiogenic therapy.

$17: 56$

61. U. Schönherr, A. Haas, M. Kralinger, K. Krepler, P. Miesbauer, K. Rigal, W. Schimetta, D. Scholda, B. Buchinger (Linz): Einjahresergebnisse der Qualitätssicherung Vitreoretinale Chirurgie bei Macular pucker - ein Projekt der Netzhautkommission der Österreichischen Ophthalmologischen Gesellschaft (Kurzvortrag) - One-year results, Quality assurance in vitreoretinal surgery for macular pucker. A project of the Retina Commission of the Austrian Ophthalmological Society

Problemstellung: Das Qualitätssicherungsprojekt „Vitreoretinale Chirurgie bei Macula pucker“ der Österreichischen Ophthalmologischen Gesellschaft wurde Anfang 2010 gestartet. Die ersten Ergebnisse werden bei der Jahrestagung der ÖOG 2011 veröffentlicht.

Methode und/oder Patienten: Alle namhaften vitreoretinalen Zentren Österreichs haben sich an diesem Projekt beteiligt und die Operations- und Verlaufdetails von Vitrektomien bei Macular pucker dokumentiert. Erfasst wurden Patientendaten, chirurgische Techniken, Operationsvarianten und Komplikationen. Im Rahmen der longitudinalen Verlaufsbeobachtung werden die Visusergebnisse in Abhängigkeit vom Linsenstatus sowie Besonderheiten im Verlauf und die subjektive Zufriedenheit ermittelt. Die Verlaufskontrolle erfolgt in einem definierten Zeitraum von 6-12 Monaten postoperativ.

Ergebnisse: Die Rekrutierung für das Jahr 2010 ist zum Zeitpunkt der Abstrakterstellung noch nicht abgeschlossen. Bisher wurden die operativen Daten von 474 Vitrektomien bei Macular pucker von 14 österreichischen Zentren erfasst. Ebenfalls wurden bereits 125 Verlaufsdatensätze 6-12 Monate postoperativ erhoben. Die statistische Auswertung der Ergebnisse des Jahres 2010 wird entsprechend dem Studienprotokoll bei der Jahrestagung der Österreichischen Ophthalmologischen Gesellschaft 2011 vorliegen.

Schlussfolgerungen: Das Qualitätssicherungsprojekt der Österreichischen Ophthalmologischen Gesellschaft mit voraussichtlich über 500 Operationen von 14 österreichischen Zentren wird ein statistisches Zahlenmaterial über tatsächliche Operationsdetails, Operationsvarianten, Indikationsgrenzen, Komplikationsraten und Verlaufsergebnisse erbringen, das in vergleichbarer Form bis jetzt nicht existiert. Das Projekt ist eine hervorragende Antwort auf das gestiegene Bedürfnis eines Qualitätsnachweises in der operativen Medizin. Gefördert von der Adele Rabensteiner Forschungsgesellschaft.
Bemerkungen 
Schlüsselwörter: Qualitätssicherung, Qualitätsmanagement, Vitreoretinale Chirurgie, Vitrektomie, Macular pucker, epiretinale Membran, epiretinale Gliose, epiretinale Fibrose, Netzhautkommission, ÖOG, Österreichische Ophthalmologische Gesellschaft.

Key words: Quality assurance, quality management, vitreoretinal surgery, vitrectomy, macular pucker, epiretinal membrane, epiretinal gliosis, epiretinal fibrosis, Retina Commission, Austrian Ophthalmological Society.

62. S. Sacu, M. Georgopoulos, G. Weigert, K. Eibenberger, U. Schmidt-Erfurth (Wien): Rhegmatogene Pseudophakieablatio: Vergleich der Ergebnisse unterschiedlicher Operationstechniken (Kurzvortrag) - Pseudophacic rhegmatogenous retinal detachment: comparison of results with different surgical techniques

Problemstellung: Mit der Entwicklung der transkonjuktivalen 23-gauge Vitrektomie, Weitwinkelsystemen und anderen technischen Fortschritten sowie der Publikation positiver Studienergebnisse hat die Vitrektomie bei der Operation der Netzhautabhebung gegenüber eindellender Chirurgie („Cerclage, Plombe“) deutlich an Stellenwert gewonnen. In dieser Studie werden die anatomischen und funktionellen Ergebnisse sowie Komplikationsraten der unterschiedlichen Operationsmethoden bei primären rehegmatogenen Pseudophakie-Ablationes verglichen und den präoperativen Ausgangssituationen gegenübergestellt.

Methode und/oder Patienten: In dem Datensatz von 153 konsekutiven primären Netzhautabhebungen die zwischen 2009 und 2010 von 2 Chirurgen an der Augenklinik Wien operiert wurden, sind 57 Pseudophakie-Ablationes enthalten, die in diese Untersuchung inkludiert wurden. In der Datenbank sind die präoperativen Variablen sowie Informationen zur Operation und dem Follow-up (1 Woche, 1 Monat, 3 Monate, 6 Monte und 1 Jahr) enthalten. Die Hauptuntersuchungsvariablen sind Operationsmethode, präoperative Charakteristika der Augen, Re-Ablatio-Rate, Komplikationen und funktionelles Ergebnis.

Ergebnisse: Von den 57 konsekutiv erfassten primären rhegmatogene Pseudophakieamotionen wurden 12 (21\%) mit Cerclage, 28 (49\%) mit Vitrektomie mit Gasfüllung, 10 (18\%) mit kombinierter Cerclage und Vitrektomie, 5 (9\%) mit Vitrektomie mit Silikonölfüllung und 4 mit Kryopexie und intravitrealer Gasinjektion behandelt. In 6 Fällen (11\%) musste aufgrund einer Re-Ablatio ein zweiter operativer Eingriff durchgeführt werden. Nach Cerclage war dies in 2 von 12 Fällen notwendig, nach Vitrektomie mit Gasfüllung in 2 von 28 Fällen (17\% versus $7 \%$ ). Präoperativ war die Fovea in $70 \%$ der Fälle bereits abgehoben, eine PVR Grad B oder C lag in 65\% der Fälle vor. Der Zusammenhang der Defektlokalisation mit der Auswahl chirurgischen Methode und der Komplikationshäufigkeit wird präsentiert. Weiters wird die intraoperative 360-Grad Endolaser-Applikation diskutiert.

Schlussfolgerungen: Bei der Pseudophakie-Ablatio sehen wir eine deutliche Tendenz weg von der Cerclage hin zu der primären transkonjuktivalen 23-gauge Vitrektomie. Die geringere Reoperationsrate nach der Vitrektomie stellt einen wesentlichen Vorteil gegenüber der Cerclage dar.

Schlüsselwörter: Pseudophake Netzhautablösung, Cerclage, 23-Gauge Vitrektomie, Re-Ablatio.

Key words: Pseudophacic rhegmatogenous retinal detachment, encircling band, 23 gauge vitrectomy, re-operation rate. 
18:06

63. C. Schwab, A. Borkenstein, E. Lackner, D. Ivastinovic, M. Velikay-Parel (Graz): Physiologie der Glaskörperabhebung in Bezug auf Stadien, Alter und Geschlecht im OCT und Ultraschall (Vortrag) - Physiology of posterior vitreous detachment

Problemstellung: Durch das optische Kohärenz Tomographie (OCT) konnten neue Einsichten über die Rolle das vitreoretinale interface in der Pathogenese von Makula Erkrankungen wie z.B.: Makula pucker, das zystoide Makulaödem (CME) bei Diabetes, Makulalöcher oder das Irvine Gass Syndrom gewonnen werden. Mit dieser Studie wollen wir anhand einer größeren Fallzahl Aufschluss über die Häufigkeit der verschiedenen Stadien der Glaskörperabhebung bei gesunden Augen mit speziellem Bezug auf das vitreoretinale Interface, Alter und Geschlecht geben.

Methode und/oder Patienten: Es wurden 243 Augen (171 Patienten) mittels Ultraschall und OCT untersucht. Einschlusskriterien waren dabei: kurz bevorstehende Katarakt Operation, Mobilität, Einwilligungsfähigkeit, Phakie, die Abwesenheit von vitreoretinalen Erkrankungen (Makula Pucker, AMD, Diabetische Retinopathie, AVT, ZVT et al.), die Möglichkeit der Durchführung eins OCT (non posse z.B.: bei dichter Katerakt oder Hornhauttrübung) und eine Achslänge zwischen 21,5 und 24,5 mm. Die Klassifizierung des Glaskörpers folgte in: „Anliegend“, „foveale Adhäsion“, „abgehoben im OCT“ und „abgehoben im Ultraschall“. Die Erfassung der Daten erfolgte in Excel, die statistische Auswertung mittels SPSS18.0.

Ergebnisse: Insgesamt wurden 243 Augen (160 davon weibliche und 83 männliche) untersucht. Das mittlere Alter war bei den Frauen 76a, (von 44 bis 89 Jahre) bei den Männern 72a (von 46 bis 87 Jahre). Ein vollständig anliegenden Glaskörper war in 21,4\% $(n=52)$ und eine foveale Adhäsion in 21,0\% $(n=51)$ der Augen nachzuweisen, während 19,8\% $(n=48)$ im OCT und 37,9\% ( $n=92)$ im Ultraschall eine Abhebung zeigten. Bezieht man Alter und Achsenlänge in die statistische Auswertung (multinomiale Datenanalyse) ein, so konnte im Gegensatz zu anderen Studien zwischen Männern und Frauen kein signifikanter Unterschied des Glaskörperstatus nachgewiesen werden. Signifikant war jedoch die Altersabhängikeit des Glaskörperstatus (p.<0,0001), mit einem deutlichen Sprung der Abhebungshäufigkeit zwischen 65 und 70 Jahren.

Schlussfolgerungen: Unsere Ergebnisse geben detaillierte Information über die Frühstadien einer Glaskörperabhebung und Häufigkeit der in den Dekaden von 50 bis 80 . Die foveale Adhäsion ist ein Zwischenzustand und nimmt mit dem Alter ab. Alter und Achsenlänge stellen die wesentlichen Risikofaktoren da, nicht aber das Geschlecht. Auffällig ist, dass Erkrankungen, deren Ätiologie eng mit der Glaskörpergrenzmembran verbunden sind wie z.B.: idiopathische Makulalöcher, ihren Altersgipfel unmittelbar nach dem von uns beobachteten sprunghaften Anstieg der Glaskörperabhebungen haben und unterstreicht die Bedeutung der Initialstadien der Glaskörperabhebung in diesem Zusammenhang.

Schlüsselwörter: Glaskörperabhebung, Alter, Geschlecht, vitreoretinal interface.

Key words: Posterior vitreous detachment, age, gender, vitreoretinal interface.

18:15

64. A. Hochwarter, B. Wimpissinger, K. Schmid-Kubista, S. Binder (Wien): Inflammatorisches Makulaödem-Update der Pathogenese und Therapie (Vortrag) - Inflammatory macula edema - update on pathogenesis and treatment

Problemstellung: Das Makulaödem (MÖ) ist die Hauptursache für den Visusverlust bei Patienten mit Uveitis. Persistierendes MÖ, über 6 bis 9 Monate, führt zu chronischen Veränderungen der Makula mit einem Dauerverlust des Zentralvisus bei $30 \%$ den Uveitis-Patienten. Das Ziel dieser Präsentation ist es, die Entwicklung in der Pathogenese und der Therapie des entzündlichen MÖ, zusammenzufassen. Bei Beschädigung der Blut-Retina-Schranke (BRS) akkumuliert in der Retina extrazelluläre und intrazelluläre Flüssigkeit. Bei 40,5\% der Patienten
Bemerkungen 
bildet sich eine epiretinale Membran mit (9,5\%) oder ohne Vitreoretinalen-Traktion. Viele Entzündungsmediatoren sind wichtigen Faktoren für die Entstehung eines uveitischen MÖ.

Methode und/oder Patienten: Literatur-Übersichtsarbeit.

Ergebnisse: Traditonelle Standardbehandlungen der MÖ bei Uveitis sind die Gabe von lokalen und systemischen Kortikosteroiden. Ein Therapie-Schema mit intraokularen Steroiden wird besprochen. Eine intravitreale Diclofenac-Therapie beim MÖ, bringt eine Verbesserung der Sehkraft, aber korreliert nicht mit einer Abnahme der Netzhautdicke. Die vaskuläre Komponente des MÖ und die Anti-VEGF-Therapie, ihre Effektivität und vorübergehende Wirkung, werden diskutiert. TNF-Alpha-Blocker sind effektiv in der Therapie der verschiedenen entzündlichen Erkrankungen. Da stellt sich die Frage, ob sie auch effektiv beim MÖ sind, wenn es eine Folge dieser Erkrankung ist? Diagnostische und therapeutische Pars plana Vitrektomien (ppVE) bei Uveitis sind beschreiben. Was sind noch die Indikationen für eine ppVE: anliegender Glaskörper (78\%), vitreoretinale Traktionen, epiretinale Membranen?

Schlussfolgerungen: Ein Ausarbeiten von Leitfäden für eine lokale, systemische, immunosuppressive und chirurgische Behandlung des inflammatorischen MÖ ist schwer, da viele pathogenetische und ätilogische Faktoren berücksichtigt werden müssen. Die Therapie des MÖ muss nach 6 bis maximal 9 Monaten abgeschlossen sein, da danach irreversible Schäden der Netzhaut mit schwerem Vususverlust folgen.

Schlüsselwörter: Uveitis, Makulaödem, antiinflamatorische Therapie, pars plana Vitrektomie, Leitfaden.

Key words: Uveitis, macula edema, antiinflamatory treatment, pars plana vitrectomy, guidelines.

65. P. Roberts, C. Kiss, M. Munk, M. Baratsits, C. Simader, A. Montuoro, U. Schmidt-Erfurth (Wien): Sehschärfe-korrelierte OCT-Segmentierung: (Vortrag) - Mapping of Optical Coherence Tomography (OCT)-pathologies and correlation to best corrected visual acuity (BCVA)-results in subvoveal CNV-patients

Problemstellung: Areale mit unterschiedlichen Pathologien in den verschiedenen Netzhautschichten in unbehandelten CNV-Patienten sollen mit den Ergebnissen der Sehschärfe-Untersuchung verglichen werden, um mögliche Korrelationen aufzudecken. Erfassung von Veränderungen in Spectral-Domain (SD)-OCT Bildern mit Einfluss auf die Sehschärfe aufgrund von unbehandelter subfoveolärer CNV.

Methode und/oder Patienten: Insgesamt wurden 35 Augen von 35 Patienten mit unbehandelter CNV inkludiert. Zu einem Zeitpunkt vor therapeutischer Intervention wurde die Sehschärfe, gemessen in der Anzahl korrekt gelesener Buchstaben, mit OCT (Spectralis ${ }^{\circledR}$ by Heidelberg engineering)-Befunden verglichen. Jedes Schnittbild eines definierten Datensatzes wurde auf folgende Pathologien untersucht: External limiting membrane (ELM) unterbrochen, Inner Segment/ Outer Segment-Grenzschicht (IS/OS) unterbrochen, subretinale Flüssigkeit (SRF), Abhebung des retinalen Pigmentepithels [Pigment Epithelium Detachment (PED)], Drusen. Mit dem Programm „OCT toolkit v1.5“ ist es möglich, bestimmte Areale auf einzelnen OCT-Schnittbildern (B-Scans) manuell zu segmentieren. Das Programm errechnet anschließend die gesamte Fläche jeder einzelnen Pathologie (in $\mathrm{mm}^{2}$ ), welche dann auf dem en-face Bild abgebildet wird.

Ergebnisse: Die Fläche, auf welcher die ELM nicht intakt war, korrelierte signifikant $(R=0,361, p=0,033)$ mit der Sehschärfe. Die Flächen, auf denen die anderen zuvor erwähnten Pathologien (IS/OS unterbrochen, SRF, PED, Drusen) vorhanden waren, wiesen jedoch keine signifikante Korrelation mit der Sehschärfe auf. Die Integrität der ELM selbst korrelierte signifikant mit der Intaktheit der IS/OS $(R=0,641, p=0,000)$, dem Vorhandensein von $S R F(R=0,621, p=0,000)$ und Drusen $(R=0,500, p=0,002)$.
Bemerkungen 
Schlussfolgerungen: Die Intaktheit der ELM scheint ein wichtiger Marker für die Sehschärfe von Patienten mit neovaskulärer AMD zu sein. Weitere Studien müssen noch durchgeführt werden, um die Ergebnisse zu bestätigen.

Schlüsselwörter: OCT, Sehschärfe, Netzhautschichten.

Key words: OCT, BCVA, retinal layers.

66. M. Munk, C. Kiss, F. Sulzbacher, S. Eisenkölbl, S. Sacu, W. Huf, U. SchmidtErfurth (Wien): Vergleich des therapieinduzierten Anstiegs von Fern-und Nahvisus bei neovaskulärer AMD und zystischem Makulaödem (Vortrag) - Difference in therapy-induced course of near and distance visual acuity in neovascular AMD and cystoid macular edema

Problemstellung: Vergleich des therapieinduzierten Anstiegs von Fern- und Nahvisus bei neovaskulärer altersbedingter Makuladegeneration (nAMD) und zystischem Makulaödem (CME).

Methode und/oder Patienten: Bei 39 Patienten mit neu diagnostizierter subfovealer nAMD und 29 Patienten mit Uveitis-assoziertem CME wurde vor Erstintervention, 1 Tag, 1 Woche und 1, 2 und 3 Monate nach Therapiebeginn der Nahvisus mittels RadnerReading Charts (logRADscore) und der Fernvisus mittels ETDRS Reading Charts (logMAR) untersucht. nAMD Patienten wurden mit monatlichen anti-VEGF Injektionen und CME Patienten mit einer einmaligen Dosis von 4mg intravitrealem Triamcinolon (IVTA) therapiert. Verlauf von Fern-, Nahvisus und Lesegeschwindigkeit beider Krankheitsentitäten wurden analysiert und für jeden Patienten verglichen.

Ergebnisse: Bei nAMD war der Fernvisus initial 0,6 \pm 0,3, der Nahvisus 1,1 \pm 0,5. Signifikante Verbesserung nach Therapiebeginn zeigte sich beim Fernvisus 1 Woche, beim Nahvisus 1 Monat nach Erstintervention. Nach dreimonatiger Therapie verbesserte sich der Fernvisus auf 0,5 $\pm 0,3$ und der Nahvisus auf 0,8 \pm 0,5. Bei Uveitis-assoziertem CME betrug der Fernvisus initial 0,5 \pm 0,3, der Nahvisus $0,7 \pm 0,5$. Bereits 1 Tag nach IVTA zeigte sich eine signifikante Verbesserung auf $0,4 \pm 0,3$ und 0,6 $\pm 0,5$. Auch 3 Monate nach IVTA blieb die signifikante Visusverbesserung bestehen. Bei nAMD unterscheiden sich der klinische Anstieg des Fern-, und Nahvisus signifikant (p.<0.02), wohingegen IVTA-Therapie bei Uveitis-CME einen vergleichbaren Anstieg induziert.

Schlussfolgerungen: Interessanterweise führt IVTA Therapie bei CME bereits nach einem Tag, anti-VEGF Therapie bei nAMD aber erst nach einer Woche respektive einem Monat zu einem signifikanten Anstieg des Fern- bzw. Nahvisus. Es erscheint klinisch relevant und empfehlenswert, sowohl Fern, als auch Nahvisus bei Patienten mit retinalen Erkrankungen zu prüfen, um ein globales Bild der Visusbeeinträchtigung und des Therapieerfolges zu erhalten.

Schlüsselwörter: Fernvisus, Nahvisus, zystisches Makulaödem, neovaskuläre AMD, anti-VEGF, Leseleistung.

Key words: Anti-VEGF, near distance visual acuity, distance visual acuity, reading performance, cystoid macular edema, neovascular AMD.

67. S. Palkovits, M. Lasta, D. Schmidl, A. Boltz, S. Kaya, L. Schmetterer (Wien): Messung der retinalen Sauerstoff Extraktion in Patienten mit COPD (Vortrag) - Measurement of retinal oxygen saturation in patients with chronic obstructive pulmonary disease

Problemstellung: In den letzten Jahren wurden verschiedene Systeme, zur Messung der retinalen Sauerstoffsättigung entwickelt. Diese Systeme basieren auf der Analyse des vom Fundus reflektierten Lichtes. Die Bestimmung der Validität gestaltet sich schwierig, da keine Standardmethode zur Messung der Sauerstoffsättigung in den Gefäßen der menschlichen Retina vorhanden ist. In dieser Studie wurde die retinale Sauerstoffsättigung in Patienten mit reduzierter arterieller Sauerstoffsättigung, auf Grund von COPD, bestimmt, und anschließend mit den Werten der systemischen arteriellen Sauerstoffsättigung verglichen. 
Methode und/oder Patienten: Sieben Patienten mit COPD wurden in die Studie eingeschlossen. Die retinale arterielle und venöse Sauerstoffsättigung wurde mittels der spektralen Analyse eines Fundusphotos mit einer CCD Kamera (Retinaler Gefäßanalysator, Imedos, Jena, Deutschland) durchgeführt. Die Bestimmung der systemischen Sauerstoffsättigung erfolgte mit einem Pulsoxymeter.

Ergebnisse: Die systemische Sauerstoffsättigung in Patienten mit COPD war $86,7 \pm 3,7 \%$. Die retinale arterielle und venöse Sauerstoffsättigung in Patienten mit COPD waren jeweils $83,4 \pm 5,4 \%$ und $45,3 \pm 6,9 \%$. Die Korrelation zwischen der systemischen und der retinalen arteriellen Sauerstoffsättigung war hoch ( $\mathrm{r}=$ $0.81, \mathrm{P}=0.024)$.

Schlussfolgerungen: Die Daten unserer Studie zeigen eine adäquate Validität der Messung der retinalen Sauerstoffsättigung mittels des retinalen Gefäßanalysators.

Schlüsselwörter: Sauerstoffsättigung, Retina, COPD.

Key words: Oxygen saturation, retina, COPD.

68. C. Schütze, M. Bolz, R. Sayegh, B. Baumann, M. Pircher, C. Hitzenberger, U. Schmidt-Erfurth (Wien): Automatische Detektion geographischer Atrophie anhand von polarisationssensitiver Optischer Kohärenztomographie und Korrelation mit konventionellen Bildgebungsverfahren (Vortrag) - Automatic detection of geographic atrophy using polarization-sensitive spectral domain optical coherence tomography and correlation to conventional imaging techniques

Problemstellung: To investigate the ability and reproducibility of automated lesion size detection in patients with geographic atrophy (GA) using polarizationsensitive spectral domain OCT (PS-SD-OCT) and to compare findings with conventional imaging techniques such as scanning laser ophthalmoscopy (SLO) and fundus autofluorescence (FAF) imaging.

Methode und/oder Patienten: 10 eyes of 7 patients with GA were examined by PS-SD-OCT providing selective identification of the retinal pigment epithelium (RPE) layer. A novel segmentation algorithm was applied to automatically detect and quantify areas of RPE atrophy. Five examinations per eye were performed at a one day visit. The reproducibility of the segmentation algorithm was evaluated. Measured lesion sizes were correlated with manually assessed areas of GA in SLO and FAF imaging in order to validate the clinical applicability of PS-SD-OCT in GA analysis.

Ergebnisse: Mean GA lesion size of all patients measured by PS-SD-OCT was $4,71 \mathrm{~mm}^{2}( \pm 2,97)$. Mean percentage of standard deviation in all eyes in relation to mean values of all atrophic RPE lesions investigated was $4,33 \%( \pm 2,13)$. The mean variation of RPE lesion size as validated from the repeatability measurements was $0,48 \mathrm{~mm}^{2}$, implying a solid reproducibility of GA lesion size evaluation in PS-SD-OCT. GA area in SLO measured 4,49 $\mathrm{mm}^{2}( \pm 2,71)$. Mean lesion size in SLO images was $4.7 \%$ smaller than determined by PS-SD-OCT, revealing a reliable correlation. Mean GA area of all eyes assessed by FAF was $4,85 \mathrm{~mm}^{2}( \pm 2,73)$ and correlated excellent to automatically detected PS-SD-OCT images with a relative deviation of $3,0 \%$ regarding lesion size quantification.

Schlussfolgerungen: Evaluation of PS-SD-OCT demonstrated high reproducibility of lesion size determination in patients with GA. Results correlated well with conventional techniques such as SLO and FAF imaging. PS-SD-OCT may therefore be a valuable and specific imaging modality for automated determination of GA lesion size in scientific studies and clinical practice.

Schlüsselwörter: Polarisationssensitive, OCT, Geographische Atrophie, Reproduzierbarkeit, Automatische Detektion.

Key words: Polarization-sensitive, OCT (PS-SD-OCT), geographic atrophy (GA), reproducibility, automated detection.
Bemerkungen 


\section{abstracts}

Bemerkungen 


\title{
Samstag, 4. Juni 2011
}

08:00

\author{
POSTERPRÄSENTATION \\ Europahaus, Saal Europa
}

TUMORE, ORBITA, LIDER, HORNHAUT, UVEITIS

Diskussionsleitung: G. Langmann

P 1. D. Putz, G. Haas, G. Blatsios, N. Bechrakis (Innsbruck): Case report: Konjunktivales Carcinoma in situ (Poster) - Case report: Conjunctival carcinoma in situ

Problemstellung: Konjunktivale intraepitheliale Neoplasien (Conjunctival intraepithelial neoplasia - CIN) gelten als die häufigsten Tumoren der Augenoberfläche. Es wurde ein bis zu 6-facher Anstieg der Inzidenz in den letzten zwei Jahrzehnten beobachtet. Die genaue Pathogenese dieser Neoplasien ist nicht vollständig geklärt, jedoch werden unterschiedliche Risikofaktoren wie UVLicht, Rauchen, Verletzungen, HPV- und HIV-Infektionen in der Literatur diskutiert. Die definitive Diagnose wird mittels Biopsie mit anschließender histologischer Untersuchung und Klassifizierung gestellt.

Methode und/oder Patienten: Ein 57-jähriger bisher gesunder Mann gibt an seit 2008 eine Sehverschlechterung und eine zunehmende Trübung der Cornea am rechten Auge bemerkt zu haben. Zwei Jahre lang wurden regelmäßige Kontrollen beim Augenfacharzt durchgeführt. Es kam zu einer zunehmenden Verschlechterung des Hornhautbefundes. Bei der klinischen Untersuchung in unserer Ambulanz zeigte sich am rechten Auge nasal ein Pterygium, das wenige Millimeter auf die Hornhaut übergriff. An der Hornhaut rechts fiel eine homogene Trübung der temporalen Hälfte, eine vaskularisierte, papillomatös erscheinende Erhabenheit im nasal unteren Quadranten und darüber eine vaskularisierte, bindegewebig erscheinende Platte ohne genaue Abgrenzung zum Pterygium auf. Die weiteren vorderen Augenabschnitte beidseits waren unauffällig.

Ergebnisse: Es wurde am rechten Auge eine Probeexzision aus dem nasalen unteren Hornhautquadranten entnommen. Die histopathologische Untersuchung ergab die Diagnose einer konjunktivalen intraepithelialen Neoplasie II-III. Daraufhin führten wir eine umschriebene Abrasio des auffälligen Hornhautbereichs durch und kombinierten diesen Eingriff mit einer lokalen Mitomycin-C (MMC) Behandlung und Amnionmembrantransplantation. Der Patient führt regelmäßige Kontrollen in unserer Ambulanz durch, und zeigt bis dato kein Rezidiv.

Schlussfolgerungen: Die operative Entfernung eines konjuktivalen Carzinomas in situ, welches eine Ausbreitung auf die Hornhaut aufweist ist die Therapie der Wahl. Diese kann von einer Kryotherapie, einer Bestrahlung mit einem Ruthenium- oder einem Strontium-Applikator, oder von einer lokalen Applikation von Chemotherapeutika, z.B. 5-Fluoruracil, MMC oder auch Interferon- $\alpha 2 b$ um die sonst hohe Rezidivrate zu minimieren, begleitet werden. Die CIN hat im Allgemeinen eine gute Prognose. Eine histologische Untersuchung der Schnittränder und regelmäßige Kontrollen sind erforderlich.

Schlüsselwörter: Carcinoma in situ, Konjunktiva, Hornhaut, Mitomycin C.

Key words: Carcinoma in situ, conjunctiva, cornea, mitomycin c.

P 2. A. Borkenstein (Graz): Fallbericht einer Patientin mit okulär vernarbendem Pemphigoid (Poster) - Fallbericht einer Patientin mit okulär vernarbendem Pemphigoid

Problemstellung: Das okuläre vernarbende Pemphigoid ist eine seltene Autoimmunerkrankung die vornehmlich Patienten von 50-70 Jahren betrifft. Sie ist charakterisiert durch eine typischerweise bilaterale konjunktivale Fibrose, welche im weiteren Verlauf zu einer Verkürzung des Fornix, Symblepharon und Ankyloblepharon sowie zu einer Keratinisierung der Hornhaut führt. 
Methode und/oder Patienten: Eine 81-jährige Patientin wurde an unserer Klinik mit einem beidseitigen okulären Pemphigoid Stadium III sowie einem Hornhaut- Ulcus links vorstellig. Der Visus betrug beidseits Lichtempfindung. Es erfolgte die stationäre Aufnahme, Therapie des Hornhaut-Ulcus und Vorstellung an der Dermatologie zum Ausschluss einer systemischen Beteiligung. Eine Cyclophosphamid-Therapie wurde nach internistischer Rücksprache begonnen. Bei der Entlassung war der Visus auf 1/40 links angestiegen. Von Dezember 2009 bis November 2010 wurden insgesamt 10 Endoxan-Behandlungen durchgeführt. Aufgrund des eingeschränkten Allgemeinzustands der Patientin wurde nach der ersten Cyclophosphamidgabe die Dosis reduziert.

Ergebnisse: 6 Monate nach Therapiebeginn war der Visus beidseits 1/30. Trotz maximal möglicher Therapie bildete sich jedoch ein Ankyloblepharon mit sukzessiver Visusverschlechterung aus (Übergang in Stadium IV). 10 Monate nach Erstvorstellung zeigte sich ein Rezidiv des Ulcus links und 12 Monate nach Therapiebeginn war der Visus Handbewegungen rechts und Fingerzählen links.

Schlussfolgerungen: Ein okuläres vernarbendes Pemphigoid hat trotz Therapie eine eingeschränkte Prognose. Eine frühe Diagnose und adäquate Therapie ist für den Verlauf der Erkrankung essentiell.

Schlüsselwörter: Pemphogoid.

Key words: Pemphogoid.

P 3. E. Hartlieb, I. Baldissera, N. Bechrakis (Innsbruck): Carotis-Sinus cavernosus-Fistel (Poster) - Carotid-cavernous fistula

Problemstellung: Eine Carotis-Sinus cavernosus-Fistel ist eine abnormale Verbindung zwischen der Arteria carotis interna und dem Sinus cavernosus. Diese abnormale Verbindung führt auf der einen Seite zu arterialisiertem Venenblut, erhöhter Venendurchblutung und erhöhtem Venendruck, auf der anderen Seite zu einer erniedrigten arteriellen Perfusion und einem niedrigen arteriellem Druck. Man kann unterscheiden zwischen einer direkten und indirekten Fistel. Die typischen Trias der direkten Fistel sind Exophtalmus, Chemosis und ein unangenehmes, maschinenähnliches, pulssynchrones Geräusches im Kopf. Bei der indirekten Fistel zeigt sich meist ein „rotes Auge“ in unterschiedlichen Schweregraden.

Methode und/oder Patienten: Wir berichten über einen männlichen Patienten, der aufgrund eines erhöhte Augeninnendruckes von 18/26 unter Xalatan und Cosopt vom Facharzt an unsere Klinik überwiesen worden ist. In der Anamnese berichtet der Patient, daß er seit einem Monat ein gerötetes linkes Auge habe, kein Sekret. Auf eine Therapie vom Facharzt mit Antibiotika kam es zu keiner Besserung. Vor 1 Woche wurde bei einer Kontrolle beim Facharzt zusätzlich ein erhöhter Augeninnendruck am linken Auge festgestellt. Die begonnene augeninnendrucksenkende Therapie führte zu keiner Senkung des Augeninnendruckes. Zusätzlich gab der Patient an, vor ein paar Wochen Doppelbilder gesehen zu haben. Damals wurde er von augenärztlicher Seite und neurologischer Seite untersucht, jedoch wurde keine Ursache für die Lähmung festgestellt. Jetzt hat der Patient keine Doppelbilder mehr.

Ergebnisse: Bei der Untersuchung an der Spaltlampe zeigten sich am linken Auge die Gefäße der Bindehaut mit vergößertem Lumen und korkenzieherartigen Schlängelung. Der Augeninnendruck war an beiden Augen unter augendrucksenkender Therapie bei $20 \mathrm{im}$ Normbereich. Die Motilität war unauffällig. Vorest führten wir eine Therapie mit Cortison-Augentropfen durch, die augendrucksenkende Therapie wurde weiter beibehalten. Nach einer Woche Therapie kam es zu keiner Besserung der Beschwerdesymptomatik. Der abgenommene Bindehautabstich war steril. Aufgrund des Verdachtes einer arteriovenösen Fistelbildung wurde von uns eine MR-Angiographie angeordnet. Dort stellte sich ein hochgradiger Verdacht auf eine Sinus cavernosus Fistel. Zum sicheren Nachweis wurde daraufhin eine Angiographie durchgeführt. Diese bestätigte den Verdacht auf eine Sinus cavernosus Fistel. Eine neurochirurgische Abklärung erfolgte.

Schlussfolgerungen: Die Sinus cavernosus Fistel ist eine seltene Erkrankung. Bei Patienten mit einem chronischen „,rotem Auge“ beziehungsweise beim Auftreten 
einer Kombination mehrere der folgenden Symptome sollte man an die Möglichkeit einer bestehenden abnormalen Verbindung zwischen der Arterie carotis interna und dem Sinus cavernosus denken: Exophtalmus, Chemosis, unangenehmes, maschinenähnliches, pulssynchrones Geräusches im Kopf, epibulbäre Injektion, Ptosis und haemorrhagische Chemosis, erhöhter Augeninnendruck, Hornhautödem, Irisatrophie, Cataract und Rubeosis iridis, retinale Stauungszeichen mit Blutungen, Exsudationen und Stauungspapille, Augenmuskellähmung. Schlüsselwörter: Carotis-Sinus cavernosus-Fistel, „Rotes Auge“, Doppelbilder, erhöhter Augeninnendruck, Angiographie.

Key words: Carotid-cavernous fistula, redness of an eye, diplopia, increased intraocular pressure, angiography.

P 4. S. Hausberger, I. Steinbrugger, A. Haas, W. Renner, O. Schmut, A. Wedrich, M. Weger (Graz): Rolle des IL18 GCAGT Haplotyps bei Patienten mit retinalem Arterienverschluss (Poster) - Role of the IL18 GCAGT haplotype in patients with retinal artery occlusion

Problemstellung: Neben anderen konnten bislang der arterielle Hypertonus, Diabetes mellitus, Hyperlipidämien und Atherosklerose als bedeutende Risikofaktoren für den retinalen Arterienverschluss (RAO) identifiziert werden. Interleukin (IL) 18 ist ein proinflammatorisches Zytokin, welches bei vermehrter Expression eine gesteigerte Bildung von atherosklerotischen Läsionen bedingt. Träger eines GCAGT Haplotyps, welcher durch 5 IL18 Polymorphismen an der Stelle $-887,-105,+105,+183$ und +533 gebildet wird, zeigen eine verminderte Expression von IL-18. Ziel der vorliegenden Studie war es zu untersuchen, ob der IL18 GCAGT Haplotyp mit einem protektiven Effekt für das Auftreten eines RAO assoziiert ist.

Methode und/oder Patienten: An der vorliegenden retrospektiven Studie nahmen 191 Patienten (72 Frauen, 119 Männer) mit RAO und 136 Kontrollprobanden (73 Frauen, 63 Männer) teil. Der IL18 183A>G Polymorphismus wurde mittels Polymerase Kettenreaktion bestimmt. Da der GCAGT Haplotyp der Einzige ist, der das IL18 183G-Allel beinhaltet, kann durch die Bestimmung des IL18 183A>G Genpolymorphismus der GCAGT Haplotyp nachgewiesen werden.

Ergebnisse: Es zeigte sich kein signifikanter Unterschied in der Genotypenverteilung des IL18 183A>G Polymorphismus und somit des IL18 GCAGT Haplotyps zwischen Patienten mit RAO und der Kontrollgruppe (IL18 183 AA/AG/GG: 57,6\%, $38,2 \%, 4,2 \%$ vs. $54,4 \%, 36,8 \%, 8,8 \%$, p = 0,23). Hingegen konnte eine signifikant höhere Prävalenz an arteriellem Hypertonus, Diabetes mellitus und Nikotinabusus in der Gruppe mit RAO nachgewiesen werden (arterieller Hypertonus: $79,6 \%$ vs. $50,7 \%$, p. $<0,001$; Diabetes mellitus: $14,1 \%$ vs. $5,1 \%$ ( $=0,009$, Nikotinabusus: $24,1 \%$ vs. $11,8 \%, p=0,005)$.

Schlussfolgerungen: Die vorliegenden Daten lassen darauf schließen, dass der IL18 GCAGT Haplotyp keine bedeutende Rolle als Risikofaktor für den RAO spielen dürfte.

Schlüsselwörter: Retinaler Arterienverschluss, Genpolymorphismus, Risikofaktor, Interleukin 18.

Key words: Retinal artery occlusion, gene polymorphism, risk factor, interleukin 18.

P 5. G. Seidel, N. Ardjomand (Graz): Perforierendes Bulbustruama mit Zentralarterienverschluss und retiniertem Fremdkörper im Sehnerv (Poster) - Perforating bulbus trauma with central artery occlusion and foreign body trapped in the optic nerve

Problemstellung: Präsentation eines perforierenden Bulbus Traumas mit Zentralarterienverschluss und retiniertem Fremdkörper im Sehnerv.

Methode und/oder Patienten: Fallbericht

Ergebnisse: Ein 43 Jahre alter Mann präsentierte sich mit Hammer-Meißel-Verletzung mit Eintrittspforte 1,5 mm superior paralimbal. Orbitaröntgen und CT 
zeigten einen Fremdkörper im Nervus opticus. Während der Vitrektomie wurde eine zentrale Ischämie der Netzhaut und Austrittspforte durch den Sehnervenkopf sichtbar. Obwohl es nicht möglich war den Fremdkörper zu entfernen, verbesserten sich 2 Monate nach dem Trauma sowohl das Gesichtsfeld, als auch der Visus von Handbewegungen auf 0,5. Es bestanden keine Anzeichen einer Siderose.

Schlussfolgerungen: Im Sehnerven retinierte Fremdkörper sind sehr selten und in Kombination mit einer Arterienstenose bis dato noch nicht beschrieben. Trotz des massiven Traumas war das Outcome besser als erwartet.

Schlüsselwörter: Trauma, Zentralarterienverschluss, Fremdkörper, Sehnerv. Key words: Trauma, central artery occlusion,foreign body, optic nerve.

P 6. A. Dimmer (Innsbruck): Das orbitale Kompartmentsyndrom: ein wichtiger ophthalmologischer Notfall Vergleich dreier klinischer Verläufe mit unterschiedlichem Outcome (Poster) - Orbital compartment syndrome: an important ophthalmological emergency Comparison of three clinical courses with different outcome

Problemstellung: Das orbitale Kompartmentsyndrom (OCS) ist ein seltener aber wichtiger, weil visusbedrohender ophthalmologischer Notfall, welcher durch einen akuten Anstieg des intraorbitalen Gewebedruckes gekennzeichnet ist. Retrobulbäre Hämatome unterschiedlicher Genese stellen die häufigste Ursache des OCS dar. Durch den erhöhten intraorbitalen Druck kann es zu Schädigungen des Auges sowie intrakonaler Strukturen kommen, welche eine irreversible Erblindung zur Folge haben können. Daher kommt der raschen klinischen Diagnosestellung zur sofortigen therapeutischen Intervention eminente Bedeutung zu. Analysiert werden Ursachen, Pathophysiologie, Klinik, Diagnostik, Therapieoptionen und prognostische Faktoren.

Methode und/oder Patienten: retrospektive Fallanalyse dreier klinischer OCS Verläufe mit unterschiedlichem Outcome und Vergleich mit der aktuellen Literatur. Fall 1: 70-jährige Patientin mit traumatisch bedingtem Retrobulbärhämatom und konsekutiver Amaurose bei Orbitabodenfraktur nach Skiunfall; ophthalmologische Erstvorstellung und Diagnose eines OCS nach über $24 \mathrm{~h}$ posttraumatisch, konservative Therapie; Fall 2: 36-jähriger Patient mit traumatisch bedingter Jochbein- und Orbitabodenfraktur; OCS mit Amaurose nach kieferchirurgischem Eingriff; Diagnose eines OCS 3h postoperativ; chirurgische Dekompression; Fall 3: 20-jähriger Patient mit traumatisch bedingter Orbitabodenfraktur; OCS mit Amaurose nach kieferchirurgischem Eingriff; Diagnose eines OCS 10min postoperativ; chirurgische Dekompression;

Ergebnisse: Fall 1: persistierende Amaurose bei später Diagnosestellung über $24 \mathrm{~h}$ und rein konservativer Therapie Fall 2: persistierende Amaurose bei verzögerter Diagnosestellung über $3 \mathrm{~h}$ und chirurgischer Dekompression Fall 3: vollständige Visusrehabilitation bei sofortiger Diagnosestellung und chirurgischer Dekompression

Schlussfolgerungen: Die Diagnose eines OCS kann rein klinisch gestellt werden. Eine frühzeitige Erkennung und sofortige orbitale Dekompression sind notwendig um einen permanenten Visusverlust zu verhindern. Als wichtiger prognostischer Faktor erweiset sich das Zeitintervall zwischen dem Erstauftreten der klinischen Symptomatik und der orbitalen Dekompression. Ein „globe tenting“ Phänomen im CT ist mit einer schlechten Visusprognose assoziiert.

Schlüsselwörter: Orbitales Kompartment Syndrom, orbitaler Notfall, Retrobulbärhämatom, orbitale Dekompression, globe tenting.

Key words: Orbital compartment syndrome, retrobulbar hemorrhage, orbital decompression, orbital emergency, globe tenting.

P 7. E. Pichler-Rubenzucker, U. Pichler, F. Buder (Steyr): Operative Therapie des beidseitigen konvergenten Retraktionssyndromes Stilling-Türk-Duane vom Typ I - ein Fallbericht (Poster) - surgical therapy of the bilateral Duane's retraction syndrome type I - a case report 
Problemstellung: Eine 13-jährige Patientin stellte sich an unserer Sehschule mit der Fragestellung der operativen Therapie des Einwärtsschielens bei bekanntem beidseitigen Retraktionssyndrom Stilling-Türk-Duane vor. Die Bewegungseinschränkung sowie die Schielstellung bestanden seit Geburt.

Methode und/oder Patienten: Präoperativ zeigte sich bei deutlicher Abduktionseinschränkung und Retraktion in Adduktion beidseits eine Esotropie von 16 pdpt in der Ferne kombiniert mit einer -VD von 6 pdpt bei einem unkorrigierten Visus von 1,0 beidseits. Da dem Retraktionssyndrom eine Fehlinnervation äußerer Augenmuskeln zugrunde liegt, ist eine Heilung durch einen operativen Eingriff welcher Art auch immer nicht möglich. Die Beseitigung der störenden Esotropie in Primärposition als Ziel der Operationsplanung wurde definiert und mit Patientin und Mutter besprochen. Nach Literaturrecherche wurde die Rücklagerung des M. rectus medialis beidseits in Allgemeinanästhesie durchgeführt.

Ergebnisse: Mit der genannten Operationstechnik konnte in Primärposition Parallelstand erreicht werden. Der postoperative Covertest in Primärposition in der Ferne ergab eine geringgradige Divergenz von -2 pdpt kombiniert mit einer -VD von 3 pdpt.

Schlussfolgerungen: Die Bewegungseinschränkung der Augen beim Retraktionssyndrom Stilling-Türk-Duane kann mit einer Muskelrücklagerung nicht behoben werden, aber sowohl Binokularfunktion und kosmetisch störender Schielwinkel in Primärposition können durch eine Operation verbessert werden.

Schlüsselwörter: Retraktionssyndrom Stilling-Türk-Duane, Esotropie in Primärposition, operative Therapie.

Key words: Duane's retraction syndrome, esotropia, surgical therapy.

P 8. J. Wagner, B. Langner- Wegscheider, A. Haas, A. Wedrich (Graz): Fallbericht einer akuten posterioren multifokalen plakoiden Pigmentepitheliopathie (Poster) - Case report of a patient with acute posterior multifocal placoid pigment epitheliopathy

Problemstellung: Die akute posteriore multifokale plakoide Pigmentepitheliopathie (APMPPE) ist eine seltene entzündliche Erkrankung. Ein Infekt kann ihr vorausgehen und sie kann mit zahlreichen Krankheiten (unter anderem mit Tuberkulose oder mit einer ZNS- Angiitis) einhergehen. Sie ist durch weissliche, plakoide Läsionen am hinteren Pol sowie durch eine frühe Hypo- und eine späte Hyperfluoreszenz in der Fluoreszenzangiographie charakterisiert. Die Wirksamkeit einer systemischen Steroidtherapie ist umstritten.

Methode und/oder Patienten: Eine 17-jährige Patientin wurde wegen einer seit 5 Tagen bestehenden Sehstörung vorstellig. Es zeigte sich eine, bis auf eine rezidivierende Angina, unauffällige Anamnese; es bestanden keine Kopfschmerzen und auch keine Parästhesien. Der Visus betrug beidseits 1,25. Vorderkammer und Glaskörper waren zellfrei; im Glaskörper waren jedoch Trübungen. In der Makula zeigten sich rechts mehr als links weiße chorioretinale Läsionen, welche teilweise konfluierend waren. Die Fluoreszenzangiographie ergab eine frühe Hypo- und eine späte Hyperfluoreszenz. Aufgrund des morphologischen Befundes wurde die Diagnose APMPPE gestellt.

Ergebnisse: Nach einer Woche war der Visus auf beidseits 0,4 gesunken, beide Makulae zeigten konfluierende weisse Läsionen. Bei der nächsten Kontrolle 2 Wochen nach Erstvorstellung betrug der Visus 0,5/0,6 und es wurde eine systemische Steroidtherapie eingeleitet um die Narbenbildung einzuschränken. 4 Wochen nach Erstvorstellung war der Visus auf 0.6 beidseits angestiegen und es zeigte sich beidseits eine beginnende Pigmentierung der Läsionen. 6 Wochen nach Erstvorstellung war der Visus auf 0,8/0,7, binokular 1,0, angestiegen. Der Fundus zeigte beidseits abgeheilte, pigmentierte Läsionen.

Schlussfolgerungen: APMPPE ist eine seltene Erkrankung mit moderater bis guter Visusprognose. Der Nutzen einer Steroidtherapie ist umstritten.

Schlüsselwörter: Uveitis, APMPPE.

Key words: Uveitis, APMPPE. 
P 9. K. Weiss, B. Langner-Wegscheider, M. Weger, Y. El-Shabrawi (Klagenfurt): IL-23R Genpolymorphismen in Uveitis assoziierten Erkrankungen (Poster) - Genetic variants of the IL-23R in uveitis associated disease

Problemstellung: Interleukin-23 spielt eine entscheidende Rolle in der Entwicklung pathogener T-Zellen, die Zytokine produzieren und so zur nichtinfektiösen Form der Uveitis führen. Studien haben gezeigt, dass durch einen Austausch eines Nukleotids im Interleukin-23 Rezeptor entzündliche Systemerkrankungen wie Morbus Chron, Psoriasis, Spondylitis ankylosans gehäuft vorkommen. Ein Zusammenhang von Genpolymorphismen im IL-23 Rezeptor und dem Auftreten von nichtinfektiösen intraoculären Entzündungen und deren systemisch assoziierten Erkrankungen wurde untersucht.

Methode und/oder Patienten: Patienten mit einer Iridozyklitis oder Uveitis intermedia, und eine Kontrollgruppe mit HLA B27 negativen und HLA B27 positiven Patienten wurde auf eine Assoziation mit SNP (single nucleodid polymorphism) untersucht. Zehn IL-23R SNPs wurden typisiert: rs 7517847, rs 2201841, rs 10889677, rs 1495965 , rs 11209232 , rs 1343151 , rs 10489629 , rs 11465804 , rs 11209026, rs 1004819. Systemerkrankungen wie Morbus Bechterew, undifferenzierte Spondylarthrophatie, juvenile Polyarthritis wurden miterfasst. Statistik: Chi-Quadrattest, Kontingenz Koeffizient wurde angewandt, wenn 1 Zelle weniger als 5 Zählungen hatte.

Ergebnisse: Es zeigte sich wie in der Literatur eine Signifikanz bei undifferenzierten Spondylarthropathien, aber nicht bei Uveitiden. Dies gibt einen Hinweis für eine unterschiedliche Rolle von Interleukin 23 in beiden Erkrankunsformen.

Schlussfolgerungen: Es wurde gezeigt, dass es durch einen Austausch eines Nukleotids im IL-23R das Auftreten von undifferenzierten Spondylarthropathie gehäuft vorkommt. Durch die niedrige Patientenzahl und ausgewählte SNPs sind weitere Untersuchungen nötig um die Zusammenhänge zwischen dem Auftreten einer nichtinfektiösen Uveitis und systemischen entzündlichen Erkrankungen zu erkennen.

Schlüsselwörter: Interleukin-23, undifferentierte Spondylarthrophatie, Uveitis. Key words: Interleukin-23, undifferentiated spondylarthropathy, uveitis.

P 10. M. Strasser-Schrattenthaler (Innsbruck): Diagnose und Behandlung eines Tolosa-Hunt-Syndroms - Fallbericht (Poster) - Diagnosis and successful treatment of the Tolosa-Hunt-Syndrome - case report

Problemstellung: Ein 62-jähriger Patient stellt sich mit starken Kopfschmerzen sowie Doppelbildern beim Blick nach rechts in der Notfallambulanz vor. Die Schmerzen bestehen bereits seit fünf Tagen, die Doppelbilder sind erst neu aufgetreten. Bisher hat der Patient verschiedenste Schmerzmittel eingenommen, worauf nur geringe Linderung der Schmerzen eintritt. Der Patient war bisher ophthalmologisch und allgemeinmedizinisch unauffällig.

Methode und/oder Patienten: Der neurologische Status, das Blutbild und die Schilddrüsenparametern sind unauffälliges, der Borrelienbefund ist negativ, das Schädel-CT zeigt keine Auffälligkeiten und in der Lumbalpunktion kann eine Subarachnoidalblutung ausgeschlossen werden. Klinisch deuten die Symptome auf eine N. abducens Parese rechts hin, worauf mit einer intravenösen Schmerztherapie begonnen wird. Nach zwei weiteren Tagen verstärken sich jedoch die Symptome: Schmerzen und Druckgefühl hinter dem linken Bulbus und bei Augenbewegung sowie Doppelbilder in allen Richtungen. Zusätzlich Verminderte Sensibilität in V1 und V2 des N. trigeminus sowie komplexe Motilitätsstörungen, die nicht auf eine spezielle Nervenschädigung zurückzuführen sind. Bei der MRT Untersuchung werden weder eine Raumforderungen noch ein Entzündungsherd nachgewiesen.

Ergebnisse: Ophthalmologisch zeigt sich ein starker Reizzustand links mit Exophthalmus. Der Visus (0,7 cc EB) und der Fundusbefund sind jedoch altersentsprechend.Aufgrund der Verdachtsdiagnose eines Tolosa-Hunt-Syndroms wird mit einer systemischen Kortisontherapie begonnen. Nach zweitägiger Kor- 
tisontherapie kommt es zu einer deutlichen Verbesserung der Symptomatik mit reduziertem Reizzustand, Abnahme von Exophthalmus und Sensibilitätsstörung. Weiterhin bestehen Doppelbilder in allen Richtungen sowie eine Blickparese nach links. Bei der Kontrolle nach zehn Tagen zeigt sich nur mehr ein minimaler Exophthalmus, Doppelbilder nur in Extremposition, Hyp-/Dysästhesien in V1 und V2 des N. trigeminus links bestehen weiterhin.

Schlussfolgerungen: Der beschriebene Fall zeigt einen klinisch zufriedenstellenden Verlauf. Die schrittweise Reduktion von Kortison wird gut vertragen, wobei eine Erhaltungsdosis von $8 \mathrm{mg}$ beibehalten wird. Der Patient ist nach einem Monat beschwerdefrei, die Sensibilität ist seitengleich. Bei rezidivierender Symptomatik wird eine Dosisanpassung empfohlen. Beim Tolosa-Hunt Syndrom handelt es sich um eine rein klinische Diagnose. Die sorgfältige Abklärung mit Ausschluss aller möglichen Ursachen ist verpflichtend. Im MRT können granulomatöse Entzündung im Bereich der A. carotis, des Sinus cavernosus und der Orbitaspitze aufzuweisen sein. Die Bildgebung kann aber auch wie im beschriebenen Fall unauffällig bleiben.

Schlüsselwörter: Tolosa-Hunt-Syndrom, Orbitaspitensyndrom, Sinus cavernosus, Ausschlussdiagnose.

Key words: Tolosa-Hunt-Syndrome, sinus cavernosus, diagnosis of exclusion.

\section{P 11. J. Nepp (Wien): Kausale Faktoren der Veränderungen des Lid-Wipers. (Poster) - Lid wiper changes and etiology}

Problemstellung: Der Lidwiper (LW) ist die Zone hinter der Marx-Linie, der muko-kutanen Übergangszone, welcher durch ständiges Gleiten auf der Augenoberfläche mechanischen Belastungen ausgesetzt ist. Nach Korb ist diese Zone immer ein Hinweis für Störungen des Tränenfilms. Dies ist erkennbar durch die Anfärbung mit Fluorescein bzw Lissamin-grün. Nach eigenen Beobachtungen soll nun herausgefunden werden, ob es nicht noch andere Faktoren gibt, welche den LW belasten.

Methode und/oder Patienten: In einer retrospektiven Studie werden die klinischen Belastungen mit den pathologischen Veränderungen korrelliert. Untersucht werden die Vitalfärbungen der Augenoberfläche, die Analyse des Tränenfilms mittels Lipidinterferenz und subjektiver Beschwerdesymptomatik und ätiologische Faktoren und den LW-Veränderungen gegenübergestellt. Am LW werden Vitalfärbung, Gefäßveränderungen und Teleangiektasien in 3 Schweregrade unterteilt. Statistisch wird die Pearson Korrelation angewendet.

Ergebnisse: 61 Patienten wurden konsekutiv untersucht. Die deutlichsten Veränderungen des LW zeigten sich bei Patienten mit entzündlichen 2,4 und toxischen Belastungen 2,75. Bei immunologischen Erkrankungen (Sjögren und GVHD; W: 1,75) war der Korrelationsgrad niedriger. Es fanden sich aber auch Veränderungen bei reizfreien Augen, z.B. bei Spasmen 2,0. Die beste Korrelation mit dem Tränenfilm fand sich zwischen LW und der BUT.

Schlussfolgerungen: Die Veränderungen des Lidwipers sind nicht ausschließlich Zeichen trockener Augen, sondern Symptome bei Entzündungen, toxischen und Immunologischen Belastungen, aber auch bei Spasmen zu finden. Somit ist die Wiper-Veränderung eine unspezifische Symptomatik, welche die Störungen der Augenoberfläche verdeutlicht.

Schlüsselwörter: Lid-Wiper, Ätiologie, Trockenes Auge.

Key words: Lid-wiper, etiology, dry eye.

P 12. N. Popp, N. Hirnschall, S. Tatzreiter, Y. Chen, O. Findl (Wien): Vergleich von drei Markierungsmethoden der Hornhaut vor Astigmatismuskorrektur (Poster) - Evaluation of three different cornea marking devices

Problemstellung: Ziel dieser Studie ist der Vergleich von drei unterschiedlichen kornealen Markierungstechniken vor Astimatismuskorrektur durch Implantation einer torischen Kunstlinse oder von limbalen relaxierenden Inzisionen. 
Methode und/oder Patienten: 45 Patienten wurden in drei Gruppen randomisiert und mit unterscheidlichen Markierungstechniken im Sitzen prä-operativ markiert: Markierung an der Spaltlampe mit einer Subkutan-Nadel, mit einem Pendel-Marker und Nuijts/Lane Marker mit eingebauter Wasserwaage. Danach wurden die Markierungspunkte mittels standardisierter photographischer Technik im Sitzen dokumentiert und die Abweichung der horizontalen Markierungen vom horizontalen Meridian analysiert.

Ergebnisse: Die vorläufigen Ergebnisse zeigen einen geringen Vorteil der Wasserwaage-Markierungsmethode mit einer Abweichung zum Soll-Meridian von etwas mehr als 2 Grad (SD:0.57) gegenüber etwas höheren Abweichungen mit den anderen beiden Methoden. Die Reproduzierbarkeit der photographischen Methode wird dargestellt.

Schlussfolgerungen: Alle Markierungsmethoden zeigen eine geringe Abweichung zum Soll-Meridian. Da eine Abweichung des Meridians von 5 Grad bei torischen IOL bereits eine Abschwächung der Astigmatismuskorrektur von ungefähr $17 \%$ ausmacht, ist eine möglichst genaue prä-operative Markierung von großer Wichtigkeit da die Cyclotrosion beim Liegen zwischen Patienten recht variabel ist.

Schlüsselwörter: Astigmatismus, Hornhautmarkierung, torische Linse, PendelMarker, Spaltlampen- Markierung, Nuijts/Lane Marker.

Key words: Astigmatism, corneal marking, toric lens, pendular-marker, slitlamp marking, Nuijts/Lane marker.

\section{NETZHAUT 1}

Diskussionsleitung: A. Wedrich

P 13. R. Hesse, G. Seidel, I. Steinbrugger, M. Weger, A. Haas (Graz): Anti-VEGF Behandlungsfrequenz bei exsudativer altersabhängiger Makuladegeneration über drei Jahre im Routinebetrieb. (Poster) - Frequency of AntiVEGF routine treatment for neovascular age-related macular degeneration over 3 years

Problemstellung: Die altersbedingte Makuladegeneration (AMD) ist die häufigste Erblindungsursache älterer Menschen in den Industriestaaten. Die intravitreale Applikation von Anti-VEGF Substanzen stellt die Therapie der Wahl bei exsudativer AMD dar. In dieser Arbeit soll nun erstmals die durchschnittliche Anzahl der Bevacizumab-Behandlungen pro Patienten und Auge an der Universitäts-Augenklinik-Graz in den ersten drei Behandlungsjahren im Routinebetrieb untersucht werden.

Methode und/oder Patienten: Bei dieser retrospektiven Studie wurden die Krankengeschichten von 1364 Patienten, die zwischen April 2006 und März 2009 mit der Diagnose exsudativer AMD an der Universitäts-Augenklinik-Graz aufschienen, untersucht. Ausschlusskriterien waren eine bereits erhaltene photodynamische Therapie oder Laserkoagulation am betroffenen Auge. Alle Augen, die zumindest eine Injektion im vorgegebenen Zeitraum bekamen, fielen in die Studie. Nach der ersten Dreierserie und nach jeder weiteren Bevacizumab-Behandlung wurden die Patienten vom niedergelassenen Facharzt regelmäßig kontrolliert und bei Visusverschlechterung oder klinischen Zeichen einer Aktivität für eine neuerliche Therapie zugewiesen. Die durchschnittliche Anzahl an Bevacizumab-Injektionen über drei Jahre bis zum März 2010, wurde pro Patienten und Auge statistisch erhoben.

Ergebnisse: Im Schnitt wurden im ersten Jahr 3,18, im zweiten Jahr 0,7 und im dritten Jahr 0,34 Injektionen pro Auge verabreicht. 85\% der Augen, die über drei Jahre untersucht wurden, erhielten im dritten Jahr keine Injektion mehr. Die Anzahl der Injektionen war am zweiten betroffenen Auge höher als am ersten.

Schlussfolgerungen: Diese Arbeit zeigt, dass im Routinebetrieb die Anzahl der Injektionen niedriger ist als unter Studienbedingungen. Sicherlich ist dies teilweise durch fehlende OCT- und Fluoreszenzangiographiekontrollen im nieder- 
gelassenen Bereich bedingt, die eine zeitgerechte Zuweisung der Patienten zur Wiederbehandlung ermöglichen.

Schlüsselwörter: AMD, Anzahl der Behandlungen, Anti-VEGF Therapie, 3 Jahre. Key words: AMD, number of treatments, Anti-VEGF treatment, 3 years.

P 14. G. Weigert, S. Kaya, S. Sacu, R. Werkmeister, G. Garhöfer, U. Schmidt-Erfurth, L. Schmetterer (Wien): Auswirkungen von Lutein Einnahme auf Makulapigmentdichte und Sehvermögen bei Patienten mit altersbedingter Makuladegeneration (Poster) - Effects of lutein supplementation on macular pigment optical density and visual acuity in patients with age-related macular degeneration

Problemstellung: Aus mehreren klinischen Studien gibt es Hinweise, dass verminderte Lutein Einnahme, ein Hauptbestandteil des Makulapigments, einen Risikofaktor für die Entstehung einer senilen Makuladegeneration (AMD) darstellt. In der vorliegenden Studie wurde die Hypothese getestet, dass die Einnahme von Lutein zu einer Erhöhung der Makulapigmentdichte führt. Weiters untersuchten wir die Auswirkungen auf die Sehschärfe und die Funktion der Makula.

Methode und/oder Patienten: 126 AMD Patienten (AREDS Stadien 2, 3 and 4) wurden in diese randomisierte (2:1), Placebo-kontrollierte und doppelt verblindete Studie eingeschlossen. Lutein oder Placebo wurde für 6 Monate verabreicht. Die Makulapigmentdichte wurde mittels Reflektometrie und die Sehschärfe mittels ETDRS Sehtafeln gemessen. Die Funktion der Makula wurde mittels Mikroperimetrie evaluiert.

Ergebnisse: Lutein erhöhte die Makulapigmentdichte signifikant um 27,9 $\pm 2,9 \%$ ( $\mathrm{p}<0,001 \mathrm{zu}$ Placebo). Es konnte weder eine signifikante Änderung des Funktionszustandes der Makula (MDLT = mean differential light threshold) noch der Sehschärfe (VA = visual acuity) festgestellt werden. Es konnte lediglich ein tendenzieller Anstieg für beide Parameter detektiert werden (MDLT: $p=0,096 \mathrm{zu}$ Placebo, VA: $p=0,070 \mathrm{zu}$ Placebo). Allerdings stellten wir eine signifikante Korrelation zwischen dem Anstieg der Makulapigmentdichte nach 6 Monaten und dem Anstieg der MDLT nach 6 Monaten $(r=0,25, P=0,027)$ sowie dem Anstieg der Makulapigmentdichte nach 6 Monaten und dem Anstieg der Sehschärfe nach 6 Monaten $(\mathrm{r}=0,27, \mathrm{P}=0,013)$ fest.

Schlussfolgerungen: Die vorliegende Studie zeigt, dass Lutein die Makulapigmentdichte, gemessen mit einer objektiven Methode, erhöht. Die Korrelation zwischen der Veränderung der Makulapigmentdichte und der Veränderung der Sehschärfe und der MDLT deutet darauf hin, dass Patienten, die einen starken Anstieg der Makulapigmentdichte zeigen, auch in Bezug auf die visuelle Funktion profitieren.

Schlüsselwörter: AMD, Makulapigmentdichte, Lutein.

Key words: AMD, macular pigment optical density, lutein.

P 15. N. Bechrakis, G. Charitoudis, G. Willerding, A. Joussen, R. Schuster, U. Keilholz (Innsbruck): Änderung der Anzahl von zirkulierenden Tumorzellen bei Patienten mit Aderhautmelanom, vor und nach primärer Behandlung (Poster) - Change in the amount of circulating tumor cells in patients with uveal melanoma, before and after primary treatment

Problemstellung: Ziele der Studie waren sowohl die Ermittlung der zirkulierenden Melanomzellen in der Blutbahn von Patienten mit Aderhautmelanomen bei Erstbehandlung (Brachytherapie, Protonen-Bestrahlung, oder Enukleation) als auch bei der ersten Kontrolle nach 6 Monaten und im weiteren Verlauf, und die Beobactung des Verlaufs bzw. Metastasierung, hinsichtlich der TumorzellenCharge im Blut.

Methode und/oder Patienten: An der Studie nahmen 202 unselektierte Patienten teil, die im Jahr 2007 aufgrund eines neu entdeckten Aderhautmelanoms erstmalig behandelt worden sind. Von jedem Patienten wurde 20ml Blut vor als auch unmittelbar nach der Erstbehandlung entnommen und dann erneut 6 Mo-
Bemerkungen 
nate später. Der Nachweis von RNA-Transkripten in intakten Tumorzellen erforderte die Isolierung der RNA, die Umschreibung der mRNA in cDNA und den Nachweis der Tyrosinase- und MelanA/Martl-cDNA mittels RT-PCR.

Ergebnisse: Am Tag der operativen Behandlung waren 153 Patienten (85,0\%) negativ und 27 (15,0\%) positiv für die Expression des Tyrosinase und/oder des MelanA/Martl Gens. 6 Monate später waren 139 Patienten $(87,4 \%)$ negativ und $20(12,6 \%)$ positiv für die entsprechende Gene. In diesem Zeitraum sind 15 Patienten positiv konvertiert, 19 Patienten sind negativ konvertiert, 108 Patienten waren immer negativ und 3 waren immer positiv. Bis zum November 2009 trat eine Metastasierung bei 7 von 108 (6,5\%) ständig negativen Patienten, bei 2 von $14(14,2 \%)$ positiv gewordenen Patienten, bei 3 von 19 (15,8\%) negativ gewordenen Patienten und bei 2 von 3 (66,7\%) ständig positiven Patienten.

Schlussfolgerungen: Die Änderung des Prozentsatzes der Patienten mit Tumorzellen in ihrer Blutbahn war in unserer Studie nicht statistisch signifikant, entweder wegen einer diskontinuierlichen Dissemination oder einer Tumorzellzahl nahe der unteren Nachweisgrenze. Mit den in dieser Studie angewanden Methoden konnte kein Einfluss der Lokaltherapie auf die Tumorzelldessimination festgestellt werden. Demgegenüber konnte jedoch trotz bislang kurzer Nachbeobachtungszeit eine erhöhte Metastasierungshäufigkeit bei denjenigen wenigen Patienten, die sich in mehr als einer Messung als positiv erwiesen haben, dokumentiert werden, sowie eine niedrige Metastasierungsrate bei den Patienten mit durchweg negativen Blutproben.

Schlüsselwörter: Uveales Melanom, zirkulierende Tumorzellen, Tyrosinase, Melan A.

Key words: Uveal melanoma, circulating tumor cells, Tyrosinase, Melan A.

P 16. M. Theurl, N. Bechrakis, M. Kralinger (Innsbruck): Behandlung einer traumatischen submakulären Blutung mittels Vitrektomie und rtPA versus keine Behandlung. Ein Fallbericht. (Poster) - Treatment of a traumatic submacular hemorrhage with vitrectomy and rtPA versus no treatment. A case report

Problemstellung: Bisher existieren in der Literatur nur einzelne Fallberichte zur Behandlung traumatischer subretinaler Blutungen mittels Vitrektomie und rtPA Eingabe, wobei deutliche Visusverbesserungen berichtet wurden. Submakuläre Blutungen treten vor allem im Rahmen einer exsudativen AMD auf. Auch eine Entfernung des Blutes aus der Makula mittels Vitrektomie und recombinant tissue plasminogen activator (rtPA) resultiert in diesen Fällen oft nicht in einem guten postoperativen Visus. Wir präsentieren hier den klinischen Verlauf zweier Fälle von traumatischen submakulären Blutungen bei jungen Patienten, wobei in einem Fall eine Vitrektomie mit rtPA Eingabe stattfand.

Methode und/oder Patienten: Fall A: Ein 35-jähriger Patient wurde nach einem stumpfen Bulbustrauma mit submakulärer Blutung stationär aufgenommen, der Visus betrug 0,1. Wir entschlossen uns zu einer Behandlung mittels 23G Pars-plana-Vitrektomie, subretinaler rtPA Eingabe $(40 \mu \mathrm{g}$, Konzentration $10 \mu \mathrm{g} /$ $0,1 \mathrm{ml}$ ) und intravitrealer Lufteingabe, welche am Tag nach dem Trauma durchgeführt wurde. Fall B: Ein 18-jähriger Patient wurde nach einem stumpfen Bulbustrauma mit submakulärer Blutung stationär aufgenommen, an dem betroffenen Auge war nur Fingerzählen möglich. Wir entschlossen uns zunächst gegen eine chirurgische Intervention. In beiden Fällen wurden Fundusfotografie und OCT Scans zur Dokumentation der makulären Veränderungen durchgeführt.

Ergebnisse: Fall A: Der postoperative Visus war bereits nach 7 Wochen auf 0,6 angestiegen. Bei der letzten Folgeuntersuchung nach 9 Monaten erreichte der Patient sogar einen Visus von 1,0. Das Blut wurde durch die Operation erfolgreich aus der Fovea verdrängt. Die abschließende Fundusbeurteilung zeigt biomikroskopisch und im OCT eine unauffällige Makula, abgesehen von einer parapapillären Aderhautruptur. Fall B: Es entwickelte sich bereits kurz nach dem Trauma ein Makulaforamen, welches im Laufe einiger Wochen an Größe zunahm. Der letzte uns bekannte Visus nach 2 Monaten betrug 0,08 exzentrisch. Dem Patienten wurde eine Vitrektomie empfohlen. Der Patient war jedoch un- 
schlüssig und war anschließend zu einer weiteren Kontrolle und OP-Besprechung nicht erschienen.

Schlussfolgerungen: Die erfolgreiche Behandlung einer traumatischen submakulären Blutung mittels Vitrektomie und rtPA-Eingabe fügt sich gut ein in die wenigen anderen gleichartigen Fallberichte. Der exzellente Visusanstieg nach 9 Monaten spricht gegen eine relevante Toxizität von subretinalem rtPA in unserem ersten Fall. Der zweite Fall demonstriert, dass eine unbehandelte submakuläre Blutung bei einem zuvor Netzhautgesunden zur Desintegration der Makula und dauerhaftem Visusverlust führen kann. Kontrollierte prospektive Studien wären wichtig und notwendig, um die Wirksamkeit und Sicherheit einer Behandlung traumatischer submakulärer Blutungen mittels Vitrektomie und rtPA statistisch nachzuweisen.

Schlüsselwörter: Submakuläre Blutung, Trauma, Vitrektomie, rtPA.

Key words: Submacular hemorrhage, trauma, vitrectomie, rtPA.

P 17. A. Bernhart, S. Sacu, W. Bühl, R. Dunavölgyi, A. Varga, U. Schmidt-Erfurth (Wien): Effekt der kombinierten PDT / Triamcinolon Behandlung auf die choroidale Perfusion bei neovaskulärer AMD (Poster) - Effect of combined PDT / triamcinolone therapy on choroidal perfusion in neovascular AMD

Problemstellung: Untersuchung des Effektes von Standard (SPDT) versus reduced fluence photodynamischer Therapie (RPDT) in Kombination mit intravitrealem Triamcinolonacetat (IVTA) auf die Gefäßwandintegrität und auf die choroidale Perfusion bei Patienten mit neovaskulärer altersbedingter Makuladegeneration (AMD).

Methode und/oder Patienten: In dieser prospektiven, randomisierten Studie wurden 40 Patienten mit subfovealer choroidaler Neovaskularisation (CNV) entweder mit SPDT + IVTA $\left(50 \mathrm{~J} / \mathrm{cm}^{2}, 600 \mathrm{~mW} / \mathrm{cm}^{2}\right)$ oder mit RPDT + IVTA $\left(25 \mathrm{~J} / \mathrm{cm}^{2}\right.$, $300 \mathrm{~mW} / \mathrm{cm}^{2}$ ) behandelt. Um den Therapieeffekt auf die choroidale Perfusion zu bestimmen, wurden das CNV Areal sowie die choroidale Hypoperfusion mittels Indozyaningrünangiographie zum Diagnosezeitpunkt und im Behandlungszeitraum von 12 Monaten gemessen.

Ergebnisse: Das CNV Areal war in beiden Gruppen im Vergleich zu Baseline nach 12 Monaten leicht vergrößert (SPDT + IVTA: $p=0,83$; RPDT + IVTA: $p=0,49$ ). Es zeigten sich keine signifikanten Unterschiede zwischen beiden Gruppen in Bezug auf das hypofluoreszente Areal, welches die CNV umgibt $(p>0,5)$. In der SPDT + IVTA Gruppe war dieses Areal allerdings am Tag 1 und bei Woche 1 signifikant größer verglichen mit dem Ausgangswert ( $p=0,001$ und $p=0,031)$, in der RPDT + IVTA Gruppe konnten keine signifikanten Unterschiede gezeigt werden ( $p>0,5)$. Zum Zeitpunkt Monat 1 hatten sich die die hypofluoreszenten Areale verkleinert, danach wurden in beiden Studienpopulationen keine signifikanten Unterschiede zu Baseline gefunden ( $\mathrm{p}>0,5)$.

Schlussfolgerungen: SPDT + IVTA und RPDT + IVTA Kombinationen sind hinsichtlich der Verzögerung einer CNV Progression im ersten Jahr annähernd gleich effektiv. Es zeigte sich allerdings, dass die choroidale Perfusion in frühen Stadien nach RPDT + IVTA weniger durch Perfusionsdysregulation beeinträchtigt wird als nach SPDT + IVTA.

Schlüsselwörter: PDT, IVTA, CNV, AMD, Choroidale Perfusion.

Key words: PDT, IVTA, CNV, AMD, choroidal perfusion.

P 18. U. Scheschy-Lun, R. Sayegh, C. Simader, F. Sulzbacher, S. Eisenkölbl, C. Kiss, U. Schmidt-Erfurth (Wien): ETDRS Sehtest Ergebnisse im Vergleich mit zwei automatischen Systemen bei Patienten mit und ohne Makulaerkrankungen in einer Follow-up Studie (Poster) - ETDRS visual acuity outcomes in comparison with two automatic systems in patients with and without macula diseases in a follow-up study

Problemstellung: Die Feststellung der bestkorrigierten Sehschärfe (BCVA) ist bei Patienten mit Makulaerkrankungen die am häufigsten verwendete Methode
Bemerkungen 
zur Überprüfung der Sehfunktion. In multizentrischen Studien ist das Protokoll der Early Treatment of Diabetic Retinopathy(ETDRS) Studie die Standard Methode zur Feststellung der BCVA. Die elektronische Überprüfung der Sehschärfe (EVA) wurde kürzlich in multizentrischen Studien eingeführt und der Sehtest mit dem Autorefraktometer (AR) wird häufig in Privatordinationen verwendet. Der Zweck dieser Studie war der Vergleich dieser drei Methoden bei Patienten mit Makulaerkrankungen und bei einer altersmäßig entsprechenden Kontrollgruppe ohne Netzhautveränderungen.

Methode und/oder Patienten: 75 Augen von 43 Patienten wurden mit diesen drei Methoden jeweils in derselben Reihenfolge bei drei Visits in dreimonatigem Intervall geprüft, nach vorheriger Bestimmung der optimalen Refraktion. Von den 75 Augen hatten 20 geographische Atropie (GA), verursacht durch altersbedingte Makuladegeneration (AMD), 20 choroidale Gefäßneubildung (CNV) durch AMD, 20 ein diabetisches Makulaödem (DME) und 15 Augen zeigten keine retinalen Veränderungen. Als Testinstrumente wurden ETDRS Charts, das EVA System und der Autorefraktometer verwendet. Das EVA System besteht aus einer Fernbedienung und einem Bildschirm, der jeweils einen Optotypen nach dem anderen in verschiedenen Größen zeigt. Dem Protokoll folgend blieb dabei die Sehdistanz immer drei Meter. Für jeden Visit wurden die drei VA Ergebnisse untereinander verglichen, für jede Makulaerkrankung separat.

Ergebnisse: Bei Patienten mit GA konnten bei keinem Visit signifikante Unterschiede in den BCVA Ergebnissen zwischen ETDRS, EVA und AR festgestellt werden. Bei Patienten mit CNV und DME konnten bei jedem Visit signifikante Unterschiede der BCVA Ergebnisse von ETDRS und EVA im Vergleich mit AR festgestellt werden $(\mathrm{p}<0,05)$. In der Kontrollgruppe bemerkten wir bei jdem Visit hoch signifikante Unterschiede zwischen allen drei Testmethoden. Außerdem zeigten sich bei der Kontrollgruppe bei den Follow-ups signifikante BCVA Unterschiede zwischen ETDRS, EVA und AR.

Schlussfolgerungen: Die Studie zeigt, daß Patienten mit durch GA stark verminderter Sehleistung mit allen drei Testmethoden beobachtet werden können, wobei die absolute Anzahl der gelesenen Buchstaben bei ETDRS und EVA größer war als bei AR. Bei Augen mit CNV oder DME ist eventuell für Follow-ups ein ETDRS oder EVA Test angebracht, weil deren Ergebnisse sich vom AR signifikant unterschieden. Bei der Kontrollgruppe konnten Unterschiede der BCVA zwischen allen drei Systemen festgestellt werden. Zusätzlich trat in der Kontrollgruppe bei den Follow-ups eine Verbesserung auf, wahrscheinlich durch einen Lerneffekt bedingt, der bei Patienten mit Makulaerkrankungen dem Anschein nach nicht entsteht.

Schlüsselwörter: Vergleich von Sehtestergebnissen mit ETDRS Charts, EVA Test und Autorefraktometer bei Patienten mit und ohne Makulaerkrankungen.

Key words: Comparison of visual acuity testing results using ETDRS charts, EVA test and autorefractor in patients with and without macula diseases.

P 19. U. Mayr-Sponer, C. Simader, B. Gerendas, A. Montuoro, U. Heiling, U. Schmidt-Erfurth (Wien): Evaluierung der Verlässlichkeit von Messungen mit einem neuen Testauge (SISPOT, Vienna Reading Center) für Fundusund Fluoreszenzangiographie-Kameras zum Einsatz in multizentrischen Studien (Kontrolle von Pixelgrößen-Variationen bei verschiedenen Kam (Poster) - Reliability of measurements with a new testeye (SISPOT, Vienna Reading Center) used in multicenter trials to control for pixel size variations in different camera systems

Problemstellung: To evaluate the intraoperator reliability and interoperator reliability of measurements with the Simader-Sponer-Testeye (SISPOT) Vienna Reading Center (VRC).

Methode und/oder Patienten: The SISPOT was constructed roughly following the characteristics of a Gullstrand eye, it contains a focusing screen with a circle of known size crossed by 12 radial lines. Nine different operators took 5 independent pictures with one SISPOT following a tight protocol concerning image 
taking and labelling, at 3 different cameras (FF450 Zeiss, HRAII and Spectralis Heidelberg Ing.). Pictures were evaluated digitally using a reading software to mark 24 points on each picture. Pixel size (PIXS) was then calculated automatically. The means and standard deviations (SD) were calculated for all 27 sets of measurements to evaluate repeatability. All measurements at the same camera were evaluated for interoperator reliability.

Ergebnisse: For the FF450 SD of mean values for the 9 datasets ranged from 0.01 to $0.02 \mu \mathrm{m} / \mathrm{p} .99 .73 \%$ of the pictures differed less then $2 \%$ in PIXS for all operators sets (3SD $68.27 \%$ ) 1 operator reached $95 \%<5 \%$. Interoperator reliability still was high $(95 \%>5 \%)$. Difficulties for testeye users may be reduced by protocol improvements. All operators using Spectralis produced pictures $(95.54 \%)$ differing less than $3 \%$ in PIXS. SD range $0.1-0.9 \mu \mathrm{m} / \mathrm{p}$. 95.54\% of the images differed less than $3 \%$ in PIXS between the operators.

Schlussfolgerungen: The SISPOT is a reliable instrument to judge pixel size of pictures taken with various camera types, independent of the operator, when a tight protocol is followed. This allows to draw conclusions about real lesion size within pictures taken with tested cameras, leading to more safety of decisions concerning patient inclusion/exclusion on digital pictures by reading centers.

Schlüsselwörter: Testauge, Pixel Größe, Funduskamera, FLA-Kamera, Vienna Reading Center.

Key words: Testeye, pixel size, variation, fundus camera, SLO-camera, Vienna Reading Center.

P 20. S. Dekanovic, R. Maier, A. Berghold, A. Wedrich (Graz): GLAS (Grazer Laser Studie) (Poster) - GLAS (Graz laser study)

Problemstellung: Die diabetische Retinopathie ist eine Spätfolge des Diabetes Mellitus und kann unbehandelt zur Erblindung führen. Der wichtigste Risikofaktor ist die Dauer der Erkrankung. Im fortgeschrittenen Stadium, der sogenannten proliferative diabetische Retinopathie, ist eine panretinale Lasertherapie indiziert um die retinalen Neovaskularisationen zu stoppen. Der Zweck dieser Studie ist eine Behandlung mit dem Pattern Scan Laser und eine Behandlung mit dem Standard Laser im Bezug auf das Schmerzempfinden und auf die sehbezogene Lebensqualität zu vergleichen.

Methode und/oder Patienten: Diese Studie ist eine prospektive randomisierte Cross-over Studie. 20 PatientInnen mit proliferativer diabetischer Retinopathie und der Notwendigkeit einer panretinalen Laserkoagulation nahmen an der Studie teil. Bei allen PatientInnen wurde ein Auge abwechselnd mit dem Standard Laser und mit dem Pattern Scan Laser in mehreren Sitzungen behandelt. Das subjektive Schmerzempfinden wurde 5 min nach der Behandlung mittels Visueller Analogskala (VAS) gemessen und es wurde vor der ersten und nach der letzten Sitzung ein Fragebogen (EQ-5D, NEI-VFQ-25) zur Lebensqualität ausgefüllt.

Ergebnisse: Die Ergebnisse werden im Rahmen der Österreichischen Ophthalmologischen Gesellschaft vorgestellt.

Schlussfolgerungen: Die Ergebnisse werden im Rahmen der Österreichischen Ophthalmologischen Gesellschaft vorgestellt.

Schlüsselwörter: Diabetische Retinopathie, panretinale Laserkoagulation, standard Laser, PASCAL Laser, Schmerz, Schmerzempfinden, VAS, visuelle Analogskala, Dolometer, sehbezogene Lebensqualität, EQ-5D, NEI-VFQ-25.

Key words: Diabetic retinopathy, panretinal photocoagulation, conventional laser, PASCAL laser, pattern scan laser, pain response, visual analog scale, Dolometer, vision-related quality of life, EQ-5D, NEI-VFQ-25.

P 21. M. Stattin, N. Bechrakis, M. Kralinger, B. Widmann-Schuchter, G. Haas, G. Blatsios (Innsbruck): Photodynamische Therapie mit Verteporfin zur Behandlung eines retinalen Hämangioms (Poster) - Photodynamic therapy with Verteporfin for treatment of retinal hemangioma

Problemstellung: Im Jänner 2010 stellt sich ein 24-jähriger Patient mit einem
Bemerkungen 
retinalen Hämangiom zur Beurteilung vor, welches seit 9 Jahren bei stabilem Befund und unverändertem Visus regelmäßig beobachtet und dokumentiert wurde, nachdem im Dezember 2009 ein Visusabfall im Rahmen der Routinekontrolle festgestellt wurde.

Methode und/oder Patienten: Anamnestisch bestanden subjektives „Verschwommen sehen" und Metamorphopsien seit etwa 4 Monaten. Ophthalmologischer Status: bestkorrigierter Visus am rechten Auge: 1,0, am linken Auge: 0,6p. Intraokulärer Druck rechts: $10 \mathrm{mmHg}$, links: $14 \mathrm{mmHg}$. Die vorderen Augenabschnitte sind beidseits unauffällig. Der Fundus des rechten Auges ist ebenfalls unauffällig. Am linken Auge zeigt sich ein großes kapilläres Hämangiom am unteren temporalen Pol, harte Exsudate bis an die Fovea reichend, der Tumor von fibrotisch weißlicher Oberfläche mit frischen dunkelroten Anteilen im oberen Bereich sowie Glaskörpertraktionen zur Basis. Weitere diagnostische Verfahren: Stratus OCT: Lipideinlagerungen, Makulaödem und zentrale epiretinale Gliose. Ultraschall: Bestimmung der Tumorgröße. Molekulargenetische Diagnostik und Bildgebung (Schädel-MRT): Ausschluss eines von-Hippel-Lindau-Syndroms.

Ergebnisse: Es wird eine photodynamische Therapie mit Visudyne mit einer Expositionszeit von 166 Sekunden durchgeführt. Postexpositionell kommt es zu einem starken Visusabfall aufgrund einer Glaskörperblutung mit seröser Ablatio zentral. Bereits nach 2 Wochen zeigt sich eine kontinuierliche Besserung des Sehvermögens mit Resorption der Blutung bei noch persistierender subretinaler Exsudation zentral. Nach 6 Monaten beträgt der bestkorrigierte Visus 0,5. Funduskopisch sind eine Verkleinerung des Tumors und eine Verringerung der harten Exsudate zu sehen.

Schlussfolgerungen: Die PDT stellt eine minimal invasive Therapieoption bei der Behandlung von retinalen Hämangiomen dar.

Schlüsselwörter: Hämangiom, Photodynamische Therapie, Verteporfin.

Key words: Hemangioma, photodynamic therapy, Verteporfin.

P 22. J. Lammer, M. Bolz, B. Wetzel, B. Baumann, M. Pircher, C. Hitzenberger, U. Schmidt-Erfurth(Wien): Reproduzierbarkeit der automatischen Quantifizierung harter Exsudate bei diabetischem Makulaödem mittels polarisationssensitiver OCT (Poster) - Reproducibility of automated quantification of hard exudates in diabetic macular edema using polarization sensitive optical coherence tomography

Problemstellung: To evaluate the specific automated quantification of hard exudates (HE) in diabetic macular edema (DME) in vivo using polarization sensitive OCT (PS-OCT).

Methode und/oder Patienten: In this study, 9 patients with DME were examined using PS-OCT technology. All of them showed HE which could be detected as polarization scrambling structures on PS-OCT scans. Each eye was examined 5 times in a row, providing comparable data in respect of image quality, position and state of the DME. Results were compared in order to assess the reproducibility and statistical reliability of the tested system. The reproducibility of the amount of HE ("total area" of HE (TA) and "total volume" of HE (TV)) which was automatically quantified on each scan was assessed and $95 \%$ confidence intervals (CI) for a change in area and volume of HE were estimated.

Ergebnisse: A robust statistically significant reproducibility could be shown for the PS-OCT device, according to all measurements of TA and TV in all patients. The overall mean \pm and mean SD of all 9 measurements was $1.29 \pm 0.33 \mathrm{~mm}^{2}$ for TA and $0.0242 \pm 0.0067 \mathrm{~mm}^{3}$ for TV. The coefficient of variation ranged from 0.10 to 0.69 (for TA) and from 0.21 to 0.59 (for TV). Agreement of measurements was assessed showing a mean difference of TA values ranging from $0.00 \mathrm{~mm}^{2}$ to $0.67 \mathrm{~mm}^{2}$ and a mean difference of TV values ranging from $0.00 \mathrm{~mm}^{3}$ to $0.01 \mathrm{~mm}^{3}$ and coefficients of repeatability of $0.76 \mu \mathrm{m}$ in the TA measurements and of $0.01 \mathrm{~mm}^{3}$ in the TV measurements.

Schlussfolgerungen: PS-OCT is a reliable tool for investigations of patients with HE due to DME. HE could be easily mapped and automatically quantified using 
new specially designed programs. The intradevice reproducibility proved to be throughout satisfactory. By that, the benefits of PS-OCT for the clinician regarding the clinical assessments of new parameters in patients with DME may be underlined.

Schlüsselwörter: OCT, DME, HE, polarisationssensitives OCT.

Key words: OCT, DME, HE, polarization-sensitive OCT.

P 23. S. Hausberger, A. Borkenstein, G. Schlemmer, G. Langmann, A. Klein-Theyer, A. Wedrich (Graz): 72-jähriger Patient mit Merkelzellcarcinom (Poster) Merkel cell carcinoma of a 72 year old male

Problemstellung: Das Merkelzellcarcinom (MZC) ist ein seltenes, aber hoch aggressives Malignom der Haut, das im Bereich lichtexponierter Areale, vor allem an der Gesichtshaut vorkommt. Aufgrund des schnellen Wachstums des Tumors sollte unverzüglich eine bioptische Diagnosesicherung und anschließende Radikalresektion durchgeführt und bei Bedarf Nachbehandlungen eingeleitet werden. Wir möchten an Hand eines Fallberichtes auf die Besonderheiten des MZC und seiner Therapiemöglichkeiten eingehen.

Methode und/oder Patienten: Wir berichten über einen 72-jährigen männlichen Patienten, der wegen des Verdachtes auf einen malignen Tumor am rechten Oberlid an unsere Klinik überwiesen wurde. Nach bioptischer Diagnosesicherung eines MZC wurde die Tumorexzision mit anschließender Lidplastik nach Cutler-Beard an unserer Klinik durchgeführt. Aufgrund der hohen Aggressivität des MZC wurde der Tumor radikal entfernt und der Patient postoperativ bestrahlt. Über einen Zeitraum von 2 Monaten trat kein Rezidiv auf, bislang sind keine Metastasen aufgetreten.

Ergebnisse: Aufgrund der hohen Malignität und der schlechten Prognose des MZC sollte bei jedem Patienten mit einer auf ein MZC verdächtigen Läsion frühzeitig eine bioptische Diagnosesicherung angestrebt werden. Vor allem bei derben, rotviolett-lividen halbkugeligen Tumoren sollte das MZC durch Biopsie als Differentialdiagnose ausgeschlossen werden.

Schlussfolgerungen: Das MZC ist ein Tumor des höheren Lebensalters (7. Lebensjahrzehnt)mit einer schlechten Prognose. Die 5-Jahres-Überlebensrate liegt zwischen 30 und 74\%, Lokalrezidive entwickeln 25-77\% der Patienten, Metastasen in regionale Lymphknoten kommen bei ca 50\% der Betroffenen vor. Therapie der Wahl ist die großzügige Exzision unter Einbeziehung der Strahlentherapie.

Schlüsselwörter: Merkelzellcarcinom, Oberlid, Chalazion, Biopsie, Malignom.

Key words: Merkel cell carcinoma, upper eyelid, chalazion, biopsy, malignoma.

P 24. K. Kriechbaum, S. Prager, M. Bolz, G. Deak, M. Kundi, U. Schmidt-Erfurth (Wien): Vergleich morphologischer und funktioneller Veränderungen der Makularegion nach einzeitiger gegen mehrzeitiger panretinaler Laserung bei Patienten mit proliferativer diabetischer Retinopathie (Poster) - Single session versus multi session panretinal photocoagulation: Morphological and functional changes of the central macular region following panretinal lasercoagulation in patients with diabetic proliferative retinopathy

Problemstellung: Ziel dieser Studie ist die Dokumentation von funktionellen und morphologischen Veränderungen der Makularegion durch Entwicklung eines Ödems nach kompletter panretinaler Laserung in nur einer Sitzung („Single Session") unter Verwendung des halbautomatischen Pascal Laser Systems (OptiMedica ${ }^{\circledR}$ Corporation, Santa Clara, CA, USA), gegen die Behandlung in mehreren Sitzungen („Multi Session“) unter Verwendung des Pascal System oder eines konventionellen Lasersystems (SupraScan ${ }^{\circledR}$, Quantel Medica, Cleremont, France) bei Patienten mit proliferativer diabetischer Retinopathie (PDRP).

Methode und/oder Patienten: In diese randomisierte prospektive Vergleichsstudie wurden bisher 37 Patienten mit Indikation zu kompletter panretinaler Laserkoagulation (1200-1500 Laserspots) eingeschlossen und mittels Randomisierung folgenden Gruppen zugeordnet: eine Gruppe erhält eine einzeitige komplette 
panretinale Laserung unter Verwendung des halbautomatischen Pascal Laser Systems, das durch Minimierung der Laser Puls Dauer eine rasche Applikation von Laserherden in kurzer Zeit erlaubt. Die Patienten der zweiten Gruppe und dritten Gruppe erhalten drei Laserbehandlungen in wöchentlichem Abstand zu je etwa 500 Spots unter Verwendung des Pascal Systems, bzw eines konventionellen Laser Systems. Vor der Lasertherapie wird die Netzhautfunktion mittels ETDRS Chart und die Netzhautmorphologie mittels OCT ermittelt. Verlaufskontrollen finden eine Woche nach Abschluss der Behandlung, danach in monatlichem Abstand statt.

Ergebnisse: Statistische Auswertung der bisher erhobenen Daten ergab keinen signifikanten Unterschied zwischen den einzelnen Gruppen und auch innerhalb der einzelnen Gruppen keine signifikante Zunahme der Netzhautdicke oder Visusverschlechterung innerhalb der ersten drei Monate nach der Laserbehandlung. In unserer Studie entwickelten innerhalb des Untersuchungszeitraumes also auch Patienten der „Single Session“ Gruppe kein signifikantes Makulaödem oder sonstige Komplikationen.

Schlussfolgerungen: Das Pascal Lasersystem (OptiMedica ${ }^{\circledR}$ Corporation, Santa Clara, CA, USA) ermöglicht durch Minimierung der Laser Puls Dauer sowohl eine rasche Applikation von Laserherden in sehr kurzer Zeit, als auch eine deutliche Verbesserung der Schmerzsymptomatik während der Laserung. Unter diesen Vorraussetzungen wird eine komplette panretinale Laserung in nur einer Sitzung möglich.

Schlüisselwörter: Panretinale Lasertherapie, Proliferative diabetische Retinopathie, Pascal Laser.

Key words: Panretinal laser therapie, proliferative diabetic retinopathy, Pascal Laser.

P 25. G. Staurenghi, A. Invernizzi, M. Oldani, L. Bonavia (Milano): 577 nm Gelber Laser versus $532 \mathrm{~nm}$ Grüner Laser bei der Behandlung von diabetischen Makulaödemen (Poster) - $577 \mathrm{~nm}$ yellow laser VS $532 \mathrm{~nm}$ green laser in the treatment of diabetic macular edema

Problemstellung: To evaluate the efficacy of the $577 \mathrm{~nm}$ yellow laser compared to the conventional $532 \mathrm{~nm}$ green laser in the treatment of diabetic macular edema

Methode und/oder Patienten: 20 patients affected by focal diabetic macular edema requiring laser treatment were evaluated with SD-OCT map, stereo fundus photography, fluorescein angiography, and randomized in two treating arms. Before the treatment BCVA was evaluated. Patients were divided in two groups. 10 patients (Group A) received grid or focal laser treatment performed with a $532 \mathrm{~nm}$ green laser. 10 patients (Group B) were treated with grid or focal laser using a $577 \mathrm{~nm}$ yellow laser. Laser intensity were recorded. The treatments were all performed by 2 skillful masked operators. Immediately after the treatment the intensity of laser spots were evaluated by fundus photography and SDOCT. One month after the treatment all the patients received a full ocular examination including BCVA evaluation, Fundus photography, SD-OCT and fluorescein angiography.

Ergebnisse: Both treatments resulted effective in reducing focal diabetic macular edema according with the literature data. Group A and Group B showed no statistically significant differences in BCVA gaining or reducing retinal thickness. However patients treated with 577 yellow laser needed lower power values in laser settings to get the whitening effect.

Schlussfolgerungen: Yellow $577 \mathrm{~nm}$ laser shows no statistically significant differences in efficacy when compared to conventional $532 \mathrm{~nm}$ green laser in DME treatment. However yellow laser needed lower power settings to reach the same effect on the treated retina. This could be explained by higher absorption by oxyhemoglobin, resulting in more effectiveness on vascular structures such as microaneurisms 
Schlüsselwörter: Retina, Laserbehandlung, Gelber Laser, Grüner Laser, diabetische Makulaödeme.

Key words: Retina, laser treatment, yellow laser, green laser, diabetic macular edema.

\section{NETZHAUT 2}

Diskussionsleitung: G. Kieselbach
Bemerkungen

P 26. G. Haas, B. Treiblmayr, E. Pöttinger, T. Heinzle, N. Bechrakis, M. Kralinger (Innsbruck): Intravitreale anti-VEGF Therapie bei Pigmentepithelabhebung (PED) (Poster) - Intravitreal anti-VEGF therapy for pigment epithelial detachment

Problemstellung: Die PED ist eine Sonderform der altersabhängigen Makuladegeneration (AMD) und kann zu einer starken Visusreduktion führen. Die antiVEGF Therapie ist gegenwärtig die Therapie der Wahl. Ziel dieser Untersuchung war die retrospektive Analyse des Behandlungseffektes der intravitrealen antiVEGF Therapie mit Bevacizumab, Ranibizumab oder Pegaptanib.

Methode und/oder Patienten: Es wurden insgesamt 22 Patienten bzw. 24 Augen mit PED, die mit anti-VEGF behandelt wurden, retrospektiv analysiert. Die Diagnose PED basierte auf einer biomikroskopischen Untersuchung und einer Bildgebung mittels Fluoreszenzangiographie (FAG) sowie optischen Kohärenztomographie (OCT). Der mittlere Behandlungszeitraum betrug 32 Monate. Untersuchungen fanden vor Behandlungsbeginn, nach der dritten intravitrealen Injektion und nach jeder folgenden Injektion statt. Die anti-VEGF Therapie wurde abhängig von den Untersuchungsergebnissen (Visus, OCT) bei Bedarf wiederholt. Es wurden fibrovaskuläre $(n=14)$ und seröse $(n=10)$ PED eingeschlossen. Drei Augen wurden mit Pegaptanib, zwei mit Ranibizumab, 16 mit Bevacizumab und drei Augen wurden mit Ranibizumab und Bevacizumab behandelt.

Ergebnisse: Der durchschnittliche base line Visus betrug 0,3 $\pm 0,14(0,05-0,8)$. Das Verhältnis von Frauen zu Männern war $77 \mathrm{zu} 23 \%$ und das Alter unserer Patienten war $76 \pm 6$ Jahre. Ein Patient nahm durchschnittlich $15 \pm 7$ (5-41) ambulante Termine für Kontrolluntersuchungen und intravitreale operative Medikamentenapplikationen in Anspruch. Der entsprechende VEGF-Inhibitor wurde beim jeweiligen Patienten im Schnitt fünfmal intravitreal verabreicht. Der Visus betrug nach drei intravitrealen Injektionen des jeweiligen anti-VEGF Medikaments 0,3 $\pm 0,16(0,05-0,8)$ und zeigte keinen Unterschied zum base line Visus. Der bei der letzten Kontrolle erhobene Visus war mit 0,4 \pm 0,17 $(0,05-1,0)$ um eine Zeile besser als der Ausgangsvisus. Es zeigte sich kein Unterschied zwischen den verschiedenen Behandlungsgruppen bzw. beiden Formen der Pigmentepithelabhebung.

Schlussfolgerungen: Die Effektivität gemessen am Visuserhalt zeigte sich nach einem durchschnittlichen Behandlungszeitraum von 32 Monaten und fünf intravitrealen anti-VEGF Injektionen bei allen drei Behandlungsgruppen. Die Abflachung der PED und Abnahme der zentralen Netzhautdicke ermittelt im OCT korrelierten nicht zwingend mit der Visusänderung. Dennoch erwies sich die OCT gemeinsam mit der biomikroskopischen Untersuchung und Visusbestimmung als geeignete Untersuchungsmethode, anti-VEGF Bedarfsinjektionen zu indizieren.

Schlüsselwörter: Seröse Pigmentepithelabhebung, fibrovaskuläre Pigmentepithelabhebung, anti-VEGF Therapie.

Key words: Serous pigment epithelial detachment, fibrovascular pigment epithelial detachment, anti-VEGF therapy.

P 27. C. Zehetner, B. Treiblmayr, M. Kralinger (Innsbruck): Photodynamische Therapie in Kombination mit Ranibizumab bei Typ 2 idiopathischen makulären Teleangiektasien (Poster) - Photodynamic therapy combined with ranibizumab in type 2 idiopathic macular teleangiectasia 
Problemstellung: To report the effects of reduced fluence photodynamic therapy combined with intravitreal ranibizumab in patients with type 2 idiopathic macular teleangiectasia (IMT).

Methode und/oder Patienten: Noncomparative, interventional, retrospective case series. Patients received photodynamic therapy with reduced fluence and intravitreal ranibizumab within 24 hours for type 2 idiopathic macular teleangiectasia without subretinal neovascularisation. Examinations included best corrected visual acuity (BCVA), fluorescein angiography (FA) and optical coherence tomography (OCT).

Ergebnisse: 5 eyes of 4 patients with type 2 IMT were studied. In all cases treatment was performed due to recent disease progression as identified by reduction of best corrected visual acuity. One eye received retreatment with this combination therapy during follow-up. After initial treatment, follow-up ranged from 3 to 22 months. Mean BCVA at baseline was $0,24 \pm 0,19$. At 3 months of follow-up BCVA increased in 3 out of 5 eyes and mean BCVA increased to $0,33 \pm 0,20$. No increase was shown in those eyes presenting with subfoveal fibrosis in OCT imaging. 2 out of these 5 eyes still had gained BCVA at the individual end points of follow-up (at 12 and 22 months respectively). 1 eye maintained stable visual acuity and two had lost vision compared to baseline. Mean BCVA at the individual end points was $0,27 \pm 0,19$. No adverse events were observed.

Schlussfolgerungen: Data from this series indicate that photodynamic therapy combined with inhibition of vascular endothelial growth factor by intravitreal ranibizumab may be proof valuable in treatment of patients with type 2 IMT and recent disease progression. Prospective study is required to confirm the beneficial effect of this treatment.

Schlüsselwörter: Juxtafoveoläre Teleangiektasien, Yannuzzi Typ 2, idiopathische makuläre Teleangiektasien, photodynamische Therapie, Ranibizumab, Lucentis.

Key words: Idiopathic macular teleangiectasia, Yannuzzi type 2, photodynamic therapy, ranibizumab, lucentis.

P 28. B. Pemp, S. Sacu, M. Burgmüller, R. Dunavölgyi, W. Bühl, C. Malbrel, U. Schmidt-Erfurth (Wien): Prospektive Verlaufsbeobachtung erworbener Farbsehstörungen bei altersbedingter Makuladegeneration mittels direkten heterochromatischen Helligkeitsvergleichs (Poster) - A prospective study of acquired colour vision anomalies in age-related macular degeneration using direct heterochromatic brightness comparison

Problemstellung: Bei altersbedingter Makuladegeneration (AMD) können erworbene Farbsehstörungen beobachtet werden, welche im Verlauf der Erkrankung zunehmen. Eine Überprüfung des Farbensehens ist daher als zusätzlicher Parameter auch in der klinischen Routine denkbar. Derzeit verfügbare Farbsehtests sind aufgrund geringer Sensitivität, beschränkter Quantifizierbarkeit, schwieriger Handhabung oder fehlender Aussagekraft für den ganzen Farbraum nur beschränkt einsatzfähig. In der vorliegenden Studie wurde der Verlauf von Farbensehstörungen bei AMD mittels direkten heterochromatischen Helligkeitsvergleichs untersucht.

Methode und/oder Patienten: 30 Patienten mit AMD (AREDS Stadien I bis IV) wurden in dieser unverblindeten prospektiven Kohortenstudie untersucht. Stadium der AMD, Sehschärfe, Kontrastsensitivität und Farbsehen wurden über einen Zeitraum von 6 Monaten überprüft. Als Farbsehtest diente ein Chromatometer (CM3, Reims Innovation en Ophthalmologie, Taissy, Frankreich). Dieses Gerät ermöglicht eine getrennte Überprüfung des rot/grün und des blau/gelb Farbsehsystems durch einen direkten Vergleich zweier Farbfelder in vier verschiedenen Helligkeitsstufen.

Ergebnisse: Der durchschnittliche Schweregrad der AMD nahm im Beobachtungszeitraum signifikant zu, während Visus und Kontrastsensitivität stabil blieben. Zu Beginn der Studie lag bei den Patienten eine Abweichung im blau/ gelb-System im Sinne einer Blauschwäche vor. Im Verlauf wurde dabei keine 
Veränderung beobachtet. Im rot/grün-System kam es hingegen nach 6 Monaten zu einer signifikanten Veränderung in Richtung einer Rotschwäche.

Schlussfolgerungen: Die Ergebnisse der Studie weisen auf eine Protanomalisierung des Farbensehens im Verlauf der AMD hin. Frühere Ergebnisse einer signifikanten Blauschwäche bei AMD konnten bestätigt werden. Die einfach anzuwendende Methode des direkten heterochromatischen Helligkeitsvergleichs könnte als zusätzlicher Verlaufsparameter bei AMD und anderen Erkrankungen, welche mit erworbenen Farbsehstörungen einhergehen, herangezogen werden. Schlüsselwörter: Farbsehstörung, altersbedingte Makuladegeneration.

Key words: Colour vision anomaly, age-related macular degeneration.

P 29. C. Thimm, J. Schimidt (Duisburg): Rasche visuelle Rehabilitation bei bilateraler radiogener seröser Ablatio (Poster) - Instant visual rehabilitation of bilateral retinal ablation due to radiation

Problemstellung: Ein 48-jähriger mit Glioblastoma multiforme entwickelte nach Tumorextirpation und adjuvant, palliativer Radio- und Chemotherapie trotz antiödematöser Steroidmedikation eine fulminante seröse Ablatio beider Augen mit Visusabfall auf Lichtscheinprojektion.

Methode und/oder Patienten: Mittels einer unverzügliche pars plana Vitrektomie mit Silikonöltamponade konnte eine rasche visuelle Rehabilitation erzielt werden.

Ergebnisse: Ein Visus vier Wochen post operativ von R/L 0,2/0,16 wurde erreicht. Bei reduzierter Prognose ad vitam konnte eine radiogen bedingte Erblindung verhindert werden.

Schlussfolgerungen: Eine sofortige pars plana Vitrektomie mit Silikonöltamponade sollte bei radiogener seröser Ablatio den Vorzug zu medikamentösen Therapieversuchen gegeben werden.

Schlüsselwörter: Visuelle Rehabilitation, radiogene seröser Ablatio.

Key words: Visual rehabilitation, retinal ablation, radiation.

P 30. M. Lasta, A. Boltz, S. Palkovits, D. Schmidl, S. Kaya, L. Schmetterer (Wien): Reproduzierbarkeit retinaler Sauerstoffsättigungsmessungen bei gesunden jungen Probanden (Poster) - Reproducibility of retinal vessel hemoglobin oxygenation saturation measurements in healthy young subjects

Problemstellung: Ischämie und eine reduzierte okuläre Duchblutung stellen einen wesentlichen Risikofaktor für die Entstehung und Progression verschiedenster okulärer Erkrankungen, wie beispielsweise Glaukom oder diabetische Retinopathie, dar. Durch neue technische Entwicklungen ist es heute möglich, die Sauerstoffsättigung in retinalen Gefäßen berührungslos, nicht invasiv und für den Patienten unbelastend zu messen. Ziel der vorliegenden Studie war es, die Lang- und Kurzzeitreproduzierbarkeit zu untersuchen.

Methode und/oder Patienten: 14 gesunde Probanden im Alter zwischen 18 und 35 Jahren wurden in die Studie eingeschlossen. Die retinale Sauerstoffsättigung wurde mittels eines kommerziell erhältlichen retinalen Gefäßanalysators (Retinal Vessel Analyzer, Imedos, Jena, Deutschland) gemessen. Dabei wird aufgrund der unterschiedlichen Absorptionsspektren von oxygeniertem und deoxygeniertem Blut aus, mit unterschiedlichen Wellenlängen erzeugten Fundus-Bildern, die Sauerstoffsättigung errechnet. Zur Evaluierung der Test/Retest-Reproduzierbarkeit dieser Messungen wurden drei konsekutive Fundusbilder verwendet. Weitere drei Bilder, 15 Minuten später, dienten zur Berechnung der Kurzzeit-Reproduzierbarkeit. Diese Messungen wurden eine Woche später wiederholt. Reproduzierbarkeitsberechnungen erfolgten mithilfe der Standardabweichung der jeweiligen Messungen, die Daten retinaler Arterien und Venen wurden separat ausgewertet.

Ergebnisse: In den retinalen Arterien wurde eine $\mathrm{SaO}_{2}$ von $91 \pm 8 \%$ (77-112\%; 40 Gefäße) gemessen, in retinalen Arterien ergab die Messung einen Wert von $49 \pm$ 9\% (32-69\%; 38 Gefäße). Die Standardabweichung zwischen den drei einzelnen Fotos betrug 3,0 $\pm 2,3 \%$ in retinalen Arterien sowie $5,4 \pm 3,7 \%$ in retinalen Venen.
Bemerkungen 
Die Kurzzeit-Standardabweichung zwischen den beiden Messzyklen betrug 2,9 \pm $2,1 \%$ in Arterien sowie $4,7 \pm 3,7 \%$ in Venen. Zwischen den einzelnen Studientagen zeigte sich eine SD von $4,0 \pm 2,8 \%$ in Arterien, während in Venen eine SD von 6,2 $\pm 4,1 \%$ gemessen werden konnte.

Schlussfolgerungen: Unsere Ergebnisse zeigen eine gute Reproduzierbarkeit der Messungen mit dieser Technik. Diese Methode wäre deshalb auch in longitudinalen Studien geeignet, die Sauerstoffsättigung retinaler Gefäße zu bestimmen. Schlüsselwörter: Sauerstoffsättigung, Retina.

Key words: Oxygen saturation, retina.

P 31. S. Eisenkölbl, C. Kiss, M. Munk, P. Roberts, F. Sulzbacher, U. Schmidt-Erfurth (Wien): Fixationsmuster von Patienten mit unbehandelter neovaskulärer AMD (Poster) - Fixation patterns in microperimetry of patients with treatment-naïve neovascular AMD

Problemstellung: Evaluierung von Fixationsmustern von Patienten mit unbehandelter neovaskulärer AMD während Mikroperimetrie und Aufzeigen von Beziehungen zu Visus und morphologischer Integrität im SD-OCT.

Methode und/oder Patienten: 32 Augen von 32 Patienten mit unbehandelter neovaskulärer AMD wurden untersucht. Die Sehrschärfe wurde mit EDTRS-Tafeln ermittelt. Zusätzlich wurde Mikroperimetrie (NIDEK microperimeter MP1) und SD-OCT (Heidelberg Spectralis OCT) durchgeführt. Die OCT B-scans wurden genutzt, um die Fovea zu lokalisieren. Die Integrität der externen Grenzschicht (ELM) und der Photorezeptorendsegmente (IS/OS) in der fovealen Region wurde in den OCT B-scans beurteilt. Desweiteren wurden die gekennzeichneten en-face Bilder des OCTs und die Fundusbilder der Mikroperimetrie überlagert und analysiert. Datensätze mit ungenügender Bildqualität zur Überlagerung wurden in der Analyse nicht beurteilt $(n=5)$.

Ergebnisse: Das mittlere Patientenalter ergab 79.2 Jahre (SD $\pm 9,1)$ und die mittlere Sehschärfe im ETDRS-Test war 54,3 (SD $\pm 11,9)$ Buchstaben. Patienten mit intakter ELM in der Fovea $(n=12)$ fixierten während $62,3 \%$ der Untersuchungszeit innerhalb eines 2 Bereichs und während 90,3\% der Zeit innerhalb 4, wogegen Patienten mit diskontinuierlicher ELM $(n=15)$ in $42,9 \%$ innerhalb 2 und in $80,5 \%$ innerhalb 4 fixierten. Die Unterschiede zwischen diesen morphologischen Gruppen waren signifikant ( $p<0,03$ in 2 und $\mathrm{p}<0,04$ in 4). Patienten mit intakter IS/OS $(n=2)$ zeigten in 55.5\% Fixation innerhalb 2 und in 91,5\% innerhalb eines 4 , wogegen Patienten mit beeinträchtigter IS/OS Integrität $(n=23)$ in $52,5 \%$ Fixation innerhalb 2 und in $84.8 \%$ innerhalb 4 zeigten. Der Korrelationskoeffizient zwischen Visus und Fixation beträgt 0,21 ( $\mathrm{p}=0,3)$ für 2 and 0,29 ( $\mathrm{p}=0,15)$ für 4 .

Schlussfolgerungen: Eine Assoziation zwischen morphologischen Veränderungen und Fixationsstabilität wurde gefunden. Die Beeinträchtigung der ELM Integrität lässt Schlussfolgerungen auf die Fixationsstabilität zu. In der vorliegenden Kohorte war die Zahl der Patienten mit intakter IS/OS zu gering um Beziehungen zur Fixation herzustellen. Es wurde keine signifikante Korrelation zwischen Visus und Fixationsmuster gefunden.

Schlüsselwörter: Fixation, AMD, Mikroperimetrie, OCT, ELM, IS/OS.

Key words: Fixation, AMD, microperimetry, OCT, ELM, IS/OS.

P 32. W. Bühl, A. Bernhart, M. Ritter, R. Dunavölgyi, G. Weigert, S. Sacu, U. Schmidt-Erfurth (Wien): Langzeit-Behandlungseffekt der Anti-VEGF-Therapie bei myoper CNV (Poster) - Long-term results of Anti-VEGF therapy for myopic CNV

Problemstellung: Ca. 2\% der Gesamtbevölkerung entwickeln eine pathologisch hohe Myopie. Zu den wichtigsten Komplikationen einer pathologischen Myopie zählt die choroidale Neovaskularisation (CNV). In solchen Fällen ist die rasche und adäquate Behandlung der CNV von großer Bedeutung für den Visus-Erhalt.

Methode und/oder Patienten: In diese klinische Studie wurden 17 Augen von 15 Patienten mit in OCT und Fluoreszenzangiographie bestätigter myoper CNV in- 
kludiert. Alle Patienten wurden mit intravitrealen Anti-VEGF-Medikamenten (Ranibizumab, Lucentis ${ }^{\circledR}$ ) behandelt. Der Therapieeffekt wurde mittels SnellenSehprobe sowie mit optischer Kohärenztomographie (Stratus- und Cirrus-OCT) jeweils vor, 1 Monat nach sowie 1 und 3 Jahre nach der Behandlung untersucht. Ergebnisse: Der mittlere Visus vor der ersten Behandlung betrug 0,23, die mittlere zentrale Netzhautdicke $244 \mu \mathrm{m}$. Nach der ersten Injektion stieg der Visus auf $0,32(\mathrm{p}<0,05)$ und nach mehrfacher Injektionsbehandlung auf 0,38 ( $\mathrm{p}<0,01)$. Eine signifikante Reduktion der zentralen Netzhautdicke (CRT) zeigte sich nur ein Monat nach der ersten Injektion $($ CRT $=200 \mu \mathrm{m})$, nicht jedoch nach $1 \mathrm{Jahr}$ $(\mathrm{CRT}=215 \mu \mathrm{m})$. Die 3-Jahres-Ergebnisse werden präsentiert.

Schlussfolgerungen: Die Behandlung der myopen CNV mit intravitrealen AntiVEGF-Medikamenten führt zu einer signifikanten Visusverbesserung, die jedoch nur bedingt mit den gemessenen Netzhautdicke-Veränderungen korreliert. Dies dürfte bei myopen Augen auf die zum Teil schlechte OCT-Scanqualität zurückzuführen sein. Aus diesem Grund stellt bei myopen Augen die Fluoreszenzangiographie eine wichtige Ergänzung bei der Diagnosestellung und zur Verlaufsbeurteilung dar.

Schlüsselwörter: AMD, CNV, Myopie, myope CNV, Anti-VEGF-Therapie, Ranibizumab.

Key words: AMD, CNV, myopia, myopic CNV, Anti-VEGF therapy, ranibizumab.

P 33. S. Mennel, T. Bertelmann, N. Kicova, A. Messerschmidt-Roth, S. Strodthoff (Marburg): Retinaler Venenverschluss und Glaskörper (Poster) - Retinal vein occlusion and vitreous

Problemstellung: Der Glaskörper wird aktuell - aufgrund seiner anatomischen Lokalisation zur Makula - als möglicher Co-Faktor in der Pathogenese verschiedenster Makulaerkrankungen untersucht. Einzelne Arbeiten lassen einen Zusammenhang zwischen Glaskörperabhebung und dem Verlauf des Makulaödems im Rahmen eines retinalen Gefäßverschlusses vermuten.

Methode und/oder Patienten: In einer Pilotstudie applizierten wir bei 10 Patienten rTPA (tissue plasminogen activator) intravitreal zur Induktion einer hinteren Glasköperabhebung. Die Verlaufskontrolle betrug 6 Monate.

Ergebnisse: Der Visus ist bei 8 von 10 Fällen innerhalb der ersten 4 Wochen angestiegen. Eine komplette Glaskörperabhebung konnte in 3 Fällen dargestellt werden.

Schlussfolgerungen: In einer retrospektiven Studie haben wir bereits gezeigt, dass bei Zentralvenenverschluss (ZVT) und Venenastverschluss (VAV) der Glaskörper häufiger anliegend ist als bei altersgesunden Kontrollaugen. Mehrere Publikationen weisen auf einen positiven therapeutischen Effekt durch die hintere Glaskörperabhebung hin. Obwohl der Visus in 8/10 Fällen innerhalb von 4 Wochen angestiegen ist, so zeigte sich eine komplette Glaskörperabhebung in nur 3 Fällen. Weitere Studien sind notwendig, um den Effekt von rTPA bei der Behandlung von Makulaödemen im Rahmen von retinalen Venenverschlüssen zu evaluieren.

Schlüsselwörter: VAV, ZVT, rTPA, TPA, enzymatische Glaskörperabhebung.

Key words: CRVO, BRVO, rTPA, TPA, enzymatic vitreolysis.

P 34. S. Kaya, K. Knogler, G. Weigert, S. Sacu, G. Garhöfer, L. Schmetterer (Wien): Abschätzung der okulären Rigidität basierend auf Messungen der Pulsamplitude mittels Pneumotonometrie und der Funduspulsationsamplitude mittels Laser Interferometrie bei Patienten mit altersbedingter Makuladegeneration (Poster) - Estimation of ocular rigidity based on measurement of pulse amplitude using pneumotonometry and fundus pulse amplitude using laser interferometry in age-related macular degeneration

Problemstellung: Theoretische Modelle weisen darauf hin, daß eine erhöhte Rigidität der Sklera eine Rolle in der Pathogenese der altersbedingten Makuladegeneration (AMD) spielen könnte. Wenige Studien haben diese biomechanischen 
Eigenschaften bisher beim Lebenden untersucht. Ziel der Studie war es, die Hypothese zu testen, ob Patienten mit altersbedingter Makuladegeneration (AMD) eine strukturell veränderte Sklerarigidität zeigen.

Methode und/oder Patienten: 44 Patienten mit AMD und eine Gruppe von 22 gesunden Vergleichsprobanden, vergleichbar in der Verteilung von Geschlecht, Alter, Augendruck und Blutdruck, wurden eingeschlossen. Die okuläre Pulsamplitude und der pulsatile Blutfluß im Auge wurden mittels Pneumotonometrie bestimmt. Die okuläre Funduspulsationsamplitude (FPA) wurde mit einem Laserinterferometer gemessen. Als Maß für die Sklerarigidität wurde der Koeffizient der okulären Rigidität (E1), berechnet aus Pulsamplitude und Funduspulsationsamplitude, basierend auf der Friedenwald Gleichung gewählt.

Ergebnisse: Keinerlei Unterschiede in Bezug auf systemischen Blutdruck und Intraokulardruck konnten zwischen den Gruppen festgestellt werden. Die Funduspulsationsamplitude FPA (AMD: 3,3 $\pm 1,2 \mu \mathrm{m}$; Kontrollgruppe: 3,9 \pm 0,8 $\mu \mathrm{m}$; $\mathrm{p}=0$,025) jedoch nicht die Pulsamplitude (AMD 2,8 \pm 0,6 mmHg; Kontrollgruppe: $3,0 \pm 1,0 \mathrm{mmHg} ; \mathrm{p}=0,34$ ) waren bei Patienten mit AMD niedriger als bei den gesunden Vergleichsprobanden. E1 war bei Patienten mit AMD $(0,91 \pm 0,11 \mathrm{AU})$ im Vergleich zur gesunden Kontrollgruppe $(0,70 \pm 0,07 \mathrm{AU} ; \mathrm{p}<0,001)$ signifikant höher.

Schlussfolgerungen: Die Daten der vorliegende Studie weisen darauf hin, dass Patienten mit AMD eine erhöhte Sklerarigidität aufweisen. Diese Ergebnisse unterstützen die Hypothese, dass bei dieser Erkrankungen die biomechanischen Eigenschaften der Augengewebe verändert sind. Ob diese Erkenntnisse kausativ am Krankheitsprozess beteiligt sind, oder als Folge der Krankheit zu werten sind, muß in longitudinalen Studien noch gezeigt werden.

Schlüsselwörter: AMD, Rigidität, Sklera.

Key words: AMD, rigdity, sclera.

P 35. A. Pollreisz, M. Georgopoulos, G. Fuchsjäger-Mayrl, W. Bühl, S. Sacu, U. Schmidt-Erfurth (Wien): SD-OCT Charakteristika bei Patienten mit therapierefraktärer exsudativer AMD (Poster) - SD OCT characteristics of patients with treatment-refractory exudative AMD

Problemstellung: Die Behandlung der exsudativen Form der altersbedingten Makuladegeneration (AMD) mittels intravitrealer Injektion von Ranibizumab wird seit einigen Jahren erfolgreich im klinischen Routinebereich durchgeführt. Studien haben jedoch gezeigt, dass ungefähr $10 \%$ aller Patienten nicht auf AntiVEGF Injektionen ansprechen. Aufgrund dieser hohen Inzidenz haben wir bei "Non-Responder" Patienten morphologische Charakteristika mittels SpectralDomain optischer Kohärenztomographie (OCT) im Detail untersucht.

Methode und/oder Patienten: In dieser retrospektiven Studie wurden 20 Augen von Patienten mit therapierefraktärer choroidaler Neovaskularisation aufgrund von AMD inkludiert und mittels Cirrus-OCT (Zeiss Meditec Inc.) analysiert. Patienten wurden als „Non-Responder“ klassifiziert, wenn nach mindestens 3 aufeinanderfolgenden Behandlungen mit Ranibizumab in monatlichem Abstand (,loading-dose“) subretinale Flüssigkeit, intraretinale Zysten, Pigmentepithelabhebung und/oder weniger als 80 Mikrometer Abnahme der zentralen Makuladicke im OCT zu beobachten war. Die OCT Bilder wurden anhand eines standardisierten Protokolls unmittelbar vor Beginn der „loading-dose“ und 4 Wochen nach der dritten Injektion, sowie bei allen Nachkontrollzeitpunkten evaluiert.

Ergebnisse: Bei der Mehrzahl an untersuchten Patienten wurde bei den Nachkontrollen eine posteriore vitreomakuläre Adhäsion beobachtet. Desweiteren konnten in der OCT-Aufnahme rekurrierende oder persistierende subretinale Flüssigkeit, Pigmentepithelabhebungen und/oder Pigmentepithelband-Unregelmäßigkeiten nachgewiesen werden. Die zentrale Makuladicke zeigte im Verlauf der Behandlung auch nach der „loading dose“ bei den meisten Patienten keine statistisch signifikante Abnahme.

Schlussfolgerungen: In unserer retrospektiv durchgeführten Studie konnten wir zeigen, dass bei Patienten mit therapierefraktärer neovaskulärer AMD die mor- 
phologischen Baseline-Charakteristika nur bedingt eine Aussage über die Prognose ermöglichen. Für eine präzise prognostische Aussage ist eine prospektive multizentrische Studie mit größerer Fallzahl und standardisierter Evaluation von morphologischen und angiographischen Parametern notwendig. In weiterer Folge ist eine Genalyse, die Erhebung der Medikamenten- und Allgemeinanamnese, sowie eine Analyse von intraokulären Zytokinen erforderlich.

Schlüsselwörter: AMD, Anti-VEGF, Non-Responder.

Key words: AMD, anti-VEGF, non-responder.

P 36. C. Glittenberg, S. Binder (Wien): OCT-basierte choroidale Blutfluss Simulation (Poster) - OCT-based choroidal blood flow simulation

Problemstellung: To ascertain the feasibility of simulating choroidal blood flow in three dimensional reconstructions of choroidal vasculature based on OCT data sets.

Methode und/oder Patienten: 512 x 128 macular cube OCT scans were collected using a Carl Zeiss Meditec Cirrus HD OCT. The data was post processed using the ImageJ software, and exported into the Osirix Diacom viewer where an isosurface mesh of the choroidal vasculature was reconstructed and exported as a VMRL into Maxon Cinema 4D 12. Using the DPIT Effex 1.5 fluid dynamics plugin, blood flow was simulated inside the reconstructed vasculature. Euler's equation for newtonian fluid was assumed for larger vessels. The Navier-Stokes equation for viscid liquids was used for smaller vessels. The resulting simulation was exported to OCTANE, a CUDA based GPU photorealistic rendering platform.

Ergebnisse: It was possible to aquire usable choroidal data on a limited amount of patients, depending on the opacity of the ocular media and the level of pigmentation of the RPE. The meshing of the choroid was succesful but suffered from background noise and artifact creation. The fluid simulation and GPU based rendering system proved to be effective.

Schlussfolgerungen: Although, in principal, it was shown to be possible to create choroidal fluid simulation based on OCT data, an OCT system with a higher choroidal penetration is necessary to collect better data., which would improve reconstruction. Additionally, the fluid simulation istelf needs to be moved to CUGA GPU processing to increase simulation speed.

Schlüsselwörter: Choroidea, Blutfluss, OCT, Simulation.

Key words: Choroid, blood flow, OCT, simulation.

P 37. M. Bolz, S. Sacu, C. Mitsch, U. Schmidt-Erfurth (Wien): Qualitätsmanagment in der gemeinsamen Betreuung von Patienten mit feuchter altersbedingter Makuladegeneration oder diabetischem Makulaödem durch eine enge Kooperation einer Universitätsklinik mit niedergelassenen FachärztInnen (Poster) - Quality management in the treatment of neovascular AMD and diabetic macular edema: Improving medical cooperation

Problemstellung: Die Einführung von Anti-VEGF Präparaten als Therapie der feuchten altersbedingten Makuladegeneration und des diabetischen Makulaödems hat eine signifikante Verbesserung der Lebensqualität der betroffenen Patienten und der Therapieoptionen für die behandelnden Ärzte mit sich gebracht. Gleichzeitig stellt diese neue Therapie, die regelmäßige Kontrolluntersuchungen und Wiederbehandlungen erfordert, eine hinsichtlich des Qualitätsmanagments organisatorische Herausforderung für intra- und extramurale Einrichtungen dar.

Methode und/oder Patienten: In einem Netzwerk der Universitätsklinik für Augenheilkunde mit niedergelassenen KollegInnen (Ophthalnet Vienna) wurden Schnittstellen in der gemeinsamen Betreuung von zunächst Anti-VEGF Patienten identifiziert und analysiert. Neue Lösungsvorschläge wurden erarbeitet, die eine direkte Kommunikation zwischen den Behandlungspartnern und eine genaue Patientendokumentation im Sinne einer engen Zusammenarbeit ermöglichen. Behandlungsstandards wurden festgesetzt, die für alle Ophthalnet-Part-
Bemerkungen 
ner klare Richtlinien für die gemeinsame Betreuung von betroffenen Patienten darstellen und auch den Zeitpunkt der Notwendigkeit einer Überweisung eines Patienten zwischen Ophthalnetpartnern festsetzen.

Ergebnisse: Die im Rahmen von Ophthalnet Vienna neu etablierten Kommunikationswege zwischen niedergelassenen Kollegen und der Universitätsklinik für Augenheilkunde Wien im Rahmen der gemeinsamen Patientenbetreuung ermöglichen eine individualisierte Therapie des einzelnen Patienten im Sinne einer bei Indikation zeitgerechten Verabreichung von Anti-VEGF Präparaten. Die enge Kooperation aller Netzwerkpartner ist Voraussetzung für die Bewältigung der durch neue Therapieoptionen gesteigerten Patientenaufkommen, ohne auf ein entsprechendes Qualitätsmanagment verzichten zu müssen. Der rege Austausch von Informationen zwischen Ophthalnetpartnern auch im Rahmen von Fortbildungsveranstaltungen und Fallbesprechungen erscheint dabei essentiell.

Schlussfolgerungen: Neue Kommunikationsmöglichkeiten zwischen behandelnden Ärzten erlauben eine Verbesserung des Qualitätsmanagments bei der Betreuung gemeinsamer Patienten mit feuchter altersbedingter Makuladegeneration oder diabetischem Makulaödem. Die Einrichtung eines Netzwerks (Ophthalnet Vienna) erscheint dabei notwendig, damit die Schnittstellen in der Betreuung definiert und gepflegt werden können. Eine Ausweitung des Netzwerks für die Betreuung von Patienten mit anderen Erkrankungen ist geplant.

Schlüsselwörter: Anti-VEGF, Ophthalnet Vienna, AMD, CNV, DME.

Key words: Anti-VEGF, ophthalnet Vienna, AMD, CNV, DME.

P 38. G. Fuchsjäger-Mayrl (Wien): Zusammenhang zwischem retinalen Blutfluss und systemischen Blutdruck bei Gesunden (Poster) - Retinal blood flow and systemic blood pressure in healthy subjects

Problemstellung: Autoregulation ist die Fähigkeit eines Gefäßbettes den Blutfluss trotz Veränderungen im Perfusionsdruck weitgehend konstant zu halten. Eine Vielzahl von Studien, bei denen entweder der Blutdruck oder der intrakuläre Druck modifiziert wurde, haben gezeigt, dass der retinale Blutfluss über einen weiten Bereich des okulären Perfusionsdrucks autoreguliert ist. Eine andere Möglichkeit Autoregulation zu beweisen ist Gruppendaten zu korrelieren, was in der vorliegenden Studie gemacht wurde.

Methode und/oder Patienten: In der vorliegenden Studie wurden 64 gesunde Probanden zwischen 18 bis 45 Jahren eingeschlossen. Ein Bidirektionales Laser Doppler Velocimeter (Oculix, Arbaz, CH) wurde zur Messung von retinaler Flussgeschwindigkeit verwendet. Der venöse Gefässdurchmesser wurde mit dem Retinalen Gefäss Analysegerät (Imedos, Jena, Deutschland) gemessen. Der retinale Blutfluss wurde durch Messung aller sichtbaren, in den Sehnerv einmündenen Venen ermittelt. Der okuläre Perfudionsdruck wurde als 2/3 vom mittleren arteriellen Druck minus dem intraokuläre Druck errechnet.

Ergebnisse: Der retinale Blutfluss war 46,2 \pm 7,4 ml/min. Der okuläre Perfusionsdruck war 49,3 $\pm 3,6 \mathrm{mmHg}$. Es gab keine signifikanten Assoziation zwischen retinalen Blutfluss und okulärem Perfusionsdruck $(r=0,04, p>0,05)$.

Schlussfolgerungen: Die Daten der vorliegenden Studie beweisen, dass der retinale Blutfluss in gesunden Personen autoreguliert ist. Das bestätigt bisherige Daten, die Interventionen wie isometrische Übungen oder künstliche Augendruckerhöhung verwendet haben. Gruppenkorrelationen zwischen retinalem Blutfluss und okulärem Perfusionsdruck stellen einen vielversprechenden Ansatz dar, um Einblick in vaskuläre Dysregulationen bei diversen Gefässerkrankungen zu bekommen.

Schlüsselwörter: Retinaler Blutfluss, Autoregulation.

Key words: Retinal blood flow, autoregulation.

P 39. F. Sulzbacher, C. Kiss, M. Funk, S. Eisenkölbl, M. Munk, S. Sacu, U. SchmidtErfurth (Wien): OCT-korrelierte funktionelle Behandlungsergebnisse bei neovaskulärer altersbedingter Makuladegeneration (Poster) - OCT-corre- 
lated functional treatment response in neovascular age-related macular degeneration

Problemstellung: Evaluierung des funktionellen Therapieerfolgs nach Ranibizumab Loadingdose bei Patienten mit neovaskulärer altersbedingter Makuladegeneration (nAMD) in Relation zum intialen OCT Befund.

Methode und/oder Patienten: Neunzehn Augen mit unbehandelter nAMD wurden mit Spectralis-OCT (SD-OCT) (Heidelberg Engineering) und Mikroperimetrie (MP) (Nidek Technologies) bei Baseline und nach 3 montalichen Ranibizumab Injektionen untersucht. Das Fundusphoto der MP wurde manuell mit dem Infrarotbild des SD-OCT mittels virtualDub (Ver.1.8.6) und Paint.NET (Ver.3.36) Software überlagert. Jeder Makulasensitvitätswert wurde an die korrespondierende Stelle im jeweiligen Schnittbild des OCT unter Verwendung von Image J Software übertragen. Im Anschluss wurden folgende SD-OCT Befunde anhand der mikroperimetrischen Ergebnisse bei Baseline und nach 3 monatlichen Injektionen untersucht: Neovaskulärer Komplex (NVC), Pigmentepithelabhebung (PED), subretinale Flüsssigkeit (SRF), intraretinale Flüssigkeit (IRF) und Zysten.

Ergebnisse: Nach 3 monatlichen Ranibizumab Injektionen erholte sich ein NVC in $51 \%$ mit einem medianen Funktionsgewinn von $5 \mathrm{~dB}$ [2dB; $11 \mathrm{~dB}]$, 49\% entwickelten eine Fibrose mit medianer Sensitivitätsänderung von 0dB [0dB; $7 \mathrm{~dB}$ ]. Eine SRF erholte sich in $92 \%$ und war mit einem medianen Funktionsgewinn von $4 \mathrm{~dB}[1 \mathrm{~dB} ; 11 \mathrm{~dB}]$ assoziiert, wobei sich eine IRF in $80 \%$ erholte in mit einem medianen Gewinn von $7 \mathrm{~dB}$ [3dB; 9dB] assoziiert war. Zysten verschwanden in $93 \%$ mit einem medianen Funktionsgewinn von 1,5dB [0dB; $6 \mathrm{~dB}]$. Eine seröse PED verflachte sich in $78 \%$ zu normaler Netzhautkonfiguration und zeigte einen medianen Funktionsgewinn von $5 \mathrm{~dB}$ [2B; 8dB], Eine fibrovaskuläre PED verflachte sich in $31 \% \mathrm{zu}$ normaler Netzhautkonfiguration und zeigte einen medianen Funktionsgewinn von 2dB [-0,5B; $4 \mathrm{~dB}]$.

Schlussfolgerungen: Initiale SD-OCT Befunde geben nützliche Informationen über ein mögliches funktionelles Therapieansprechen bei nAMD. Der größte funktionelle Benefit nach 3 monatlichen Ranibizumab Injektionen wurde nach Resorption eines Makulaödems festgestellt gefolgt von Abflachung von seröser und fibrovaskulärer PED. Bereiche von einem initialen NVC zeigen einen beträchtlichen funktionellen Benefit in der Hälfte der Fälle, die andere Hälfte entwickelt eine Fibrose ohne Funktionsgewinn.

Schlüsselwörter: Altersbedingte Makuladegeneration, Optische Koherenztomographie, Netzhautfunktion, Mikroperimetrie.

Key words: Age-related macular degeneration, optical coherence tomography, retinal function, microperimetry.

\section{INTRAOKULARLINSEN}

P 40. C. Reiter, E. Gramer, G. Gramer (Würzburg): Fehlen der zentralen Zone des Pseudoexfoliations-Materials bei Patienten mit Pseudophakie und Pseudoexfoliationssyndrom: Fallbeschreibungen (Poster) - Changed pattern of deposition of pseudoexfoliation material in patients with PEX syndrome on the anterior lens surface after intraocular lens implantation: case reports

Problemstellung: Es gibt nur wenige Einzelfallberichte über Auflagerungen von Pseudoexfoliations(PEX)-Material auf der Vorderfläche von Intraokularlinsen (IOL). PEX-Ablagerungen auf der natürlichen Linse haben eine charakteristische Anordnung mit drei voneinander abgegrenzten Zonen: eine homogene, zentrale, scheibenförmige PEX-Ablagerung und eine granuläre, streifenförmige, periphere Ablagerungszone. Beide Zonen sind durch eine klare Zone getrennt. Berichtet wird über Patienten mit charakteristischen peripheren PEX-Ablagerungen auf der IOL. Die Besonderheit ist, dass bei allen Patienten die zentrale Zone des PEXMaterials auf der Linsenvorderfläche bei Pseudophakie fehlte.
Bemerkungen 
Methode und/oder Patienten: Photodokumentation und Befunderhebung bei 4 Patienten mit PEX-Material auf der Linsenvorderfläche von intraokularen Linsen. Zur Sicherung der Assoziation mit PEX-Syndrom oder PEX-Glaukom wurde zusätzlich ein molekulargenetischer Nachweis von Mutationen im LOX-L1 Gen geführt. Die Zeitspanne zwischen Implantation der Intraokularlinse und PEXDiagnose u.a. wurde dokumentiert.

Ergebnisse: Die Photodokumentation der Anordnung des PEX-Materials auf der IOL zeigte peripher das charakteristische Bild. Die Besonderheit ist, dass bei allen Patienten die homogene runde zentrale Zone aus PEX-Material fehlte. Bei den 4 Patienten liegt der Zeitraum zwischen Linsenimplantation und Diagnose der PEX-Ablagerungen zwischen 7 und 11 Jahren. Das charakteristische Muster der streifenförmigen peripheren PEX-Ablagerungen ist bei allen Patienten vorhanden, wobei bei einem Patienten das Anfangsstadium erfasst wird und die peripheren PEX-Ablagerungen aus mehreren streifenförmigen Inseln bestehen, die später konfluieren.

Schlussfolgerungen: Das Fehlen der zentralen homogenen Zone aus PEX-Material scheint bei Pseudophakie und Ausbildung eines PEX-Syndroms oder PEXGlaukoms charakteristisch zu sein. Dies muss differentialdiagnostisch bedacht werden, um ein viele Jahre nach Linsenimplantation auftretendes PEX-Syndrom oder -Glaukom rechtzeitig zu erkennen. Das Fehlen der zentralen kreisförmigen Zone könnte durch das Fehlen der vorderen Linsenkapsel erklärbar sein. Durch Produktion von PEX-Material aus Iris und Ziliarkörper kann sich PEX-Material peripher übereinstimmend mit phaken Augen auch auf Kunststofflinsen ablagern.

Schlüsselwörter: Pseudoexfoliation, Intraokularlinse, LOX-L1, PEX-Ablagerung, Pseudoexfoliationsglaukom.

Key words: Pseudoexfoliation, intraocular lens, LOX-L1, PEX depositions, pseudoexfoliation glaucoma.

P 41. M. Strasser-Schrattenthaler, M. Kralinger, J. Troger, G. Kieselbach, (Innsbruck): Langzeitstabilität nach Implantation einer torischen one pieceIOL (Poster) - Long term stability after implantation of single-piece toric intraocular lenses

Problemstellung: Untersucht wird die Langzeitstabilität einer torischen hydrophoben Acryl-Einzelstück-IOL, welche zur Korrektur eines Hornhautastigmatismus verwendet wird.

Methode und/oder Patienten: In einer retrospektiven Untersuchung wird bei 43 Patienten mit altersbedingter Katarakt und zusätzlichem Hornhautastigmatismus von 1.00-3.00 dpt. die prä- und postoperative Sehschärfe sowie der prae und postoperative Astigmatismuswinkel verglichen. In den Kapselsack wird bei allen Patienten eine aspherische IOL, AcrySoft ${ }^{\circledR}$-Toric-IOL (SN60T3, SN60T4 oder SN60T5) implantiert. Die Kalkulation der IOL erfolgt durch den IOLMaster (teilweise Interferenzkoherenzmessung). Präoperativ werden die horizontale und vertikale Achse auf der Hornhaut markiert. Anschließend wird unter Verwendung von sphärischen und zylindrischen Gläsern der bestkorrigierte Visus (BCVA) bestimmt. Ergebnisse: Es werden 52 Augen von 43 Patienten untersucht. Die durchschnittliche Abweichung der Zylinderachse zwischen der Untersuchung am ersten postoperativen Tag und der Untersuchung 4 Wochen postoperativ beträgt $1,8 \pm 2,1$ Grad, zwischen der zweiten Untersuchung und der 12 Monatsuntersuchung werden $0,9 \pm 1,5$ gemessen. Zwischen der ersten und letzten postoperativen Messung zeigte sich keine signifikante Differenz. Auch der bestkorrigierte Visus (BCVA) ergab keine signifikante Veränderung nach einem Jahr.

Schlussfolgerungen: Die Implantation einer torischen IOL ist höchst geeignet um einen Hornhautastigmatismus zu korrigieren und zeigt sich auch nach den ersten 12 Monaten postoperativals rotatorisch stabil.

Schlüsselwörter: Katarakt, Hornhautastigmatismus, torische Einzelstück, Intraokularlinse, Langzeitstabilität.

Key words: Cataract, corneal astimatism, single-piece, toric intraocular lenses, long term stability. 
P 42. A. Scholler, B. Weingessel, P. Vecsei-Marlovits (Wien): Mikropsien nach Cataract-Chirurgie (Poster) - Micropsia after cataract surgery

Problemstellung: Als mögliche Ursache für Mikropsien werden beispielsweise Akkomodationsstörungen, Maculaerkrankungen, Ermüdung oder Epilepsie verantwortlich gemacht. Ein Auftreten nach komplikationsloser Cataractchirurgie wurde bisher nicht beobachtet. Bei einem 78 Jahre alten Patienten wurde eine HOYA AF-1 iMics1 Modell NY-60, Power 21,5 am linken Auge implantiert. Postoperativ leidet der Patient unter einem kleineren, blasseren und helleren Bild linksseitig. Weiters hat die Sehschärfe postoperativ abgenommen.

Methode und/oder Patienten: Fallstudie, ein Patient

Ergebnisse: Klinisch konnte bis jetzt keine Ursache für die Sehverschlechterung und Mikropsien gefunden werden. OCT und Gesichtsfeld ergaben keinen pathologischen Befund. Visus präoperativ •Visus s.c. 0,5 p • Visus c.c. 0,8 p • Nahvisus Jg. 1 IOL-Master präoperativ linkes Auge • Achsenlänge: 23,84 mm, R1: 8,04 mm/ 41,98 dpt x 91 , R2: 7,97 mm/ 42,35 dpt x 1 , Zyl.: 0,37 dpt x 1 Die Zielrefraktion mit der ausgewählten IOL beträgt -0,22 dpt. Autorefraktor postoperativ linkes Auge • $+0,0 \mathrm{sph}+0,75$ cyl 3 Visus postoperativ linkes Auge $\bullet$ Visus c.c. 0,5 $\bullet$ Nahvisus Jg. 3 IOL-Master postoperativ linkes Auge Achsenlänge: 23,76 mm, R1: 8,03 mm/ 42,03 dpt x 129 , R2: 7,95 mm/ 42,45 dpt x 39, Zyl.: 0,42 dpt x 39 Unsere Zielrefraktion wurde mit -0,11 dpt. bestätigt Auch der Linsenhersteller konnte keine Begründung für die bestehende Symptomatik finden.

Schlussfolgerungen: Mikropsien nach komplikationsloser Cataract-Chirurgie sind eine Seltenheit. Die Symptome dürften in diesem Fall nicht auf optische Phänomene zurückzuführen sein. Möglicherweise liegt eine neuroophthalmologische Ursache vor, die bis dato noch nicht erfasst werden konnte. Eine diesbezügliche Diagnostik wurde veranlasst.

Schlüsselwörter: Mikropsien, Cataractchirurgie, Visusverlust.

Key words: Micropsia, cataract surgery, loss of visual acuity.

P 43. G. Mylonas (Wien): Prospektive klinische Studie zur vergleichenden Untersuchung der Lentis L-312 vs. Acri.Lyc47LC Intraokularlinsen (Poster) - Prospective comparison of two acrylic-single-piece intraocular lenses: Lentis vs. Acri.Lyc 47LC

Problemstellung: Vergleich zweier hydrophiler single-piece Intraokularlinsen (IOL) die aus dem gleichen Acryl-Rohmaterial von 2 unterschiedlichen Herstellern produziert werden. Es soll der Einfluss von verschiedenen Herstellungsfaktoren (z.B. Polieren der IOL-Oberfläche, geringfügige Unterschiede im Haptikdesign, etc.) auf das klinische Ergebnis (Kapselsackstabilität, Nachstar) evaluiert werden.

Methode und/oder Patienten: In dieser bilateralen prospektiven, randomisierten Studie wurden 100 Augen von 50 Patienten mit beidseitiger altersbedingter Katarakt inkludiert. Nach Randomisierung wurde in ein Auge eine acrylic-single-piece IOL Lentis L-312 (Oculentis, Germany) und in das andere eine acrylicsingle-piece IOL Acri.Lyc47LC ${ }^{\circledR}$-IOL (Carl-Zeiss Meditec, Germany) implantiert. Visusbestimmung, Messung der Vorderkammertiefe (VKT) mit dem Visante OCT (Carl Zeiss Meditec AG), der Stabilität im Kapselsack, der Zentrierung und Beurteilung des Nachstars (PCO) an der Spaltlampe und mittels digitaler retroilluminierter Bilder ist zu standardisierten Untersuchungszeitpunkten (postoperativ nach 1 Stunde, 1 Woche, 3 und 6 Monaten) untersucht.

Ergebnisse: Mittelwert und Standardabweichung (SD) für Visus 6 Monate nach der Operation war 0,88 $\pm 0,24$ Snellen für Lentis L-312 und 0,89 $\pm 0,28$ Snellen für Acri.Lyc47LC. Mittelwert \pm SD der VKT für Lentis L-312 und Acri.Lyc47LC mit Visante OCT war 4,00 $\pm 0,34 \mu \mathrm{m}$ und 3,92 $\pm 0,32 \mu \mathrm{m}$. Die Nachstarentwicklung war für beide IOL bis zum Nachkontrollzeitpunkt von 6 Monaten sehr gering.

Schlussfolgerungen: Die beiden hydrophilen single-piece Acryllinsen zeigten vergleichbar gute funktionelle Ergebnisse, eine stabile Position im Kapselsack und nur geringe Nachstarwerte 6 Monate nach der Implantation.
Bemerkungen 
Schlüsselwörter: Intraokularlinsen, Lentis L-312 vs. Acri.Lyc47LC, Nachstar. Key words: Intraocular lenses, Lentis vs. Acri.Lyc 47LC, posterior capsular opacification.

P 44. S. Schriefl, E. Stifter, R. Menapace (Wien): Katarakt-Chirurgie mit der easyTip $^{\circledR}$ 2,2 mm Phakoemulsifikations-Spitze (Poster) - Cataract surgery with the easyTip ${ }^{\circledR} 2,2 \mathrm{~mm}$ Phacoemulsification-tip

Problemstellung: Ziel war es die Effektivität der easyTip ${ }^{\circledR}$ 2,2 mm Phakospitze für co-axiale Phakoemulsifikations-Kataraktchirurgie zu evaluieren und mit der konventionallen 20G CMP Phakospitze zu vergleichen.

Methode und/oder Patienten: An dieser prospektiven, randomisierten Studie nahmen 57 Patienten mit bilateraler Katarakt teil. Präoperativ wurde die Dichte der Linsentrübung anhand des LOCS-Gradings klassifiziert. Prä- und postoperativ wurde der best-korrigierte Visus erhoben sowie die Dichte der Hornhautendothelzellen gemessen. Bei der Operation wurde bei einer Flaschenhöhe von $70 \mathrm{~cm}$ ein Auge mit der easyTip 2,2 $\mathrm{mm}$ Spitze (mit high flow $(35 \mathrm{ml} / \mathrm{min}$ Aspiration, $500 \mathrm{mmHg}$ Vakuum)) und das Partnerauge mit der 20G CMP Spitze mit low flow ( $20 \mathrm{ml} / \mathrm{min}$ Aspiration, $500 \mathrm{mmHg}$ Vakuum) operiert. Zur Bewertung der Effektivität der Phakospitzen wurde die gesamte Operationsdauer bestehend aus „time to divide“ und „time to conquer“ mithilfe einer Stoppuhr gemessen sowie die verbrauchte Phakoenergie erhoben.

Ergebnisse: Bei vergleichbaren mittleren Katarakt-Dichten in beiden Gruppen wurde bei Operationen mit der easyTip 2,2 mm Spitze insgesamt um 31\% weniger Phakoenergie benötigt als bei Operationen mit der 20G CMP Spitze ( $p=0,003$ ). Für die Zerkleinerung des Kerns mit easyTip 2,2 mm musste im Vergleich mit 20G CMP nur $2 / 3$ der Phakoenergie genutzt werden $(p=0,001)$. Bei Verwendung der easyTip 2,2 mm Spitze wurde insgesamt nur etwa 12\% mehr Flüssigkeit verbraucht als mit 20G CMP ( $p=0,022)$. Die eine Woche postoperativ gemessenen Visusergebnisse und die prozentuelle Endothelzellenverluste waren nicht unterschiedlich.

Schlussfolgerungen: Durch die Verwendung der mit high flow betriebenen easyTip 2,2 mm Spitze konnte die für die Kernaufarbeitung erforderliche Phakoemulsifikationsenergie signifikant verringert werden. Der um 12\% erhöhte Flüssigkeitsverbrauch war gemessen an der um 75\% höheren Pumpdrehzahl gering. Bei einer Verwendung von üblichen Flaschenhöhen von bis zu $100 \mathrm{~cm}$ ließe sich die Durchflussrate und damit auch die Effizienz noch steigern um das bereits sehr geringe „OP-Trauma“ weiter zu minimieren.

Schlüsselwörter: Phakoemulsifikation, Katarat, Phakospitzen, easyTip 2,2 mm, 20G CMP.

Key words: Phacoemulsification, cataract, phacoemulsification-tip, easyTip 2,2 mm, 20G CMP.

P 45. K. Scherer, H. Guth, T. Graf, C. Rieder (Karlsruhe): Wissensbasiertes Informations- und Assistenzsystem in der Kataraktchirurgie (Poster) - A knowledge based assistant system for cataract surgery

Problemstellung: Im Rahmen der Ophthalmochirurgie werden jährlich über 7 Mio. Kataraktoperationen weltweit durchgeführt, davon allein in Deutschland über 600.000/Jahr mit steigender Tendenz. In Abhängigkeit unterschiedlicher Operationstechniken und der Anwendung unterschiedlicher Diagnostikverfahren ist in Anbetracht der Vielfalt von Intraokular-Implantaten eine patientenbezogen optimale IOL-Implantation unter den zur Zeit gültigen Standards wünschenswert, aber nicht trivial. Es besteht der Bedarf, auch über die reine Diagnostik hinaus vorhandene Informationen gesamtheitlich zu korrelieren und auch die postoperativ abgeschätzten Ergebnisse bereits im Vorfeld in die Entscheidungsfindung mit einzubeziehen.

Methode und/oder Patienten: Wissensbasierte Ansätze (Expertensysteme) bieten sich in der Informationstechnik an, wenn menschliches Fachwissen incl. Hintergrundwissen und Schlußfolgerungsfähigkeit maschinell zur Verfügung 
gestellt werden sollen. Insbesondere kann heterogenes verteiltes ophthalmologisches Fachwissen strukturiert und konsistent verwaltet und dem Arzt bei Bedarf komfortabel zur Verfügung gestellt werden, um ihn bei seiner Urteilsbildung zu unterstützen. Hierzu wurde mit Informatikmethoden menschliches Wissen in Form von Fakten und Regeln in formale, Computer ablauffähige Strukturen transformiert. Es wurde eine Abbildung zwischen natürlichsprachiger und maschinell verarbeitbarer Information geschaffen. Weiterhin wurden Methoden entwickelt, welche die Integration von Multimediainformation in Form von Bildern, Video und Audio ermöglichen.

Ergebnisse: Es ist ein Softwareprototyp entwickelt, mit dem in einer hierarchisch strukturierten Wissenbank neues Wissen leicht und natürlichsprachig integriert werden kann. Weiterhin werden semantische Zusammenhänge und induzierte Konsequenzen mit graphischen Repräsentationsmethoden dem Ophthalmochirurg zur Verfügung gestellt. Fachbegriffe und Informationseinheiten stehen in Verfeinerungsstrukturen zur Verfügung. Über semantische Netze wird eine intelligente Navigation kontextsensitiv ermöglicht. Die Gesamtbetrachtung der unterschiedlichen Aspekte (Diagnostik, OP-Techniken, IOL's, Patient) kann somit die Urteilsbildung unterstützen. Hierbei werden in Abhängigkeit der praeoperativen Situation und eines geplanten operativen Eingriffs die induzierten Auswirkungen erklärt. Es wird ein Prototyp eines Software-Werkzeugs vorgestellt, welches auf jedem handelsüblichen PC unter Windows lauffähig ist.

Schlussfolgerungen: Im Hinblick auf eine optimierte Katarakt-OP kann mit wissensbasierter Informationsverarbeitung dem Ophthalmochirurgen ein Assistenz-System zur Verfügung gestellt werden, welches ihn bei der Entscheidungsfindung im Rahmen der Katarakt-OP unterstützt. Durch Simulation und Berücksichtigung von induzierten Schlußfolgerungen kann somit eine modifizierte OP-Planung erfolgen. Dies ist insbesondere unter dem Aspekt der medizinischen Qualitätssicherung ein entscheidender Baustein. Ausbau und Validierung der Wissensbasis, welche sowohl das statische Wissen als auch kausale Zusammenhänge beinhaltet, muß in enger Zusammenarbeit mit Augenkliniken erfolgen.

Schlüsselwörter: Kataraktchirurgie, wissensbasierte Entscheidungsfindung, semantische Netze, Operationsplanung, Expertensystem.

Key words: Cataract surgery, knowledge based decision support, semantic network, surgical planning, expert system.

P 46. D. Pahor (Maribor): Kataraktoperationen an höchst hypermetropischen und nanophthalmischen Augen (Poster) - Cataract surgery in extreme hyperopic and nanophthalmic eyes

Problemstellung: Kataraktoperationen an höchst hypermetropischen Augen weisen eine sehr hohe Rate an intraoperativen und postoperativen Komplikationen auf. Nanophthalmus entwickelt sich häufiger bei kleineren Augen ohne okulärer Anomalie mit einer Achsellänge unter $20 \mathrm{~mm}$. Es handelt sich um eine seltene Form von angeborener Weitsichtigkeit. Mit der Studie wollten wir die Häufigkeit der extrem hypermetropischen und nanophthalmischen Augen unter unseren Patienten feststellen und die Gefahren und Komplikationen einer Katarktoperation bei Patienten mit extremer Weitsichtigkeit mit einer Achsellänge unter $21 \mathrm{~mm}$ bewerten.

Methode und/oder Patienten: Bei dieser retrospektiven Studie wurden Kranken- und Operationsberichte aller Patienten überprüft, die einer Kataraktoperation zwischen 1. Januar und 31. Dezember 2009 auf der Augenabteilung des Universitätskrankenhauses Maribor unterzogen wurden, und die Augen mit einer Achsellänge unter $21 \mathrm{~mm}$ ausgesucht. Ein Nanophthalmus wurde gemäß der kürzeren Achsellänge unter $20 \mathrm{~mm}$, flachen Vorderkammer, Weitsichtigkeit und einer mit Ultraschalluntersuchung bestimmten Skleraldicke größer als 1,7 mm diagnostiziert. Wegen Winkelblockglaukoma mussten wir noch vor der Operation auf beiden Augen eines Patienten eine Laseriridotomie durchführen. Bei allen Patienten wurde eine Clear-Cornea-Phakoemulsifikation Kleinschnitt-Kataraktoperation durchgeführt mit faltbarer Acryl-IOL-Implantation in den Kapsel- 
sack. Die Biometrie wurde mit IOL Master (Carl Zeiss Meditec, Inc) durchgeführt.

Ergebnisse: Von 1588 Augen, waren 73 Augen von 56 Patienten $(4,6 \%)$ weitsichtig. Acht Augen bei 5 Patienten (0,5\%) waren extrem weitsichtig mit einer Achsellänge unter $21 \mathrm{~mm}$. Darunter waren 2 Augen eines Patienten nanophthalmisch mit einer Achsellänge unter $19 \mathrm{~mm}$ und Netz-Aderhaut-Sklera-Dicke von 2,9 und 2,7 mm. Die Prävalenzrate des Nanophthalmus lag bei $0,2 \%$ in allen Fällen und bei 2,7\% für weitsichtige Augen. Die durchschnittliche Weitsichtigkeit betrug $+7,03 \mathrm{D}(+6,0$ bis +8,25). Die durchschnittliche Achsellänge betrug 20,2 $\mathrm{mm}(18,94$ bis 20,96) und die durchschnittliche Biometrieberechnung für IOL betrug 31,4 D $(29,0$ bis 36,0$)$. Während der Operation wurden keinerlei Komplikationen beobachtet. Bei einem nanophthalmischen Auge wurde zwei Tage nach der Operation eine milde Regenbogenhautentzündung bemerkt. Während der Nachbeobachtungszeit von 6 Monaten traten keine Komplikationen auf.

Schlussfolgerungen: Chirurgische Manipulation in der engen Vorderkammer eines extrem hyperopischen und nanophthalmischen Auges mit erhöhtem Glaskörperdruck ist ziemlich anspruchsvoll. Präoperative Untersuchungen einschließlich Ultraschalluntersuchung sind sehr wichtig um unerwartete Komplikationen vorzubeugen. Bei extrem weitsichtigen Augen mit Nanophthalmus ist die Phakoemulsifikation Kleinschnitt-Kataraktoperation ein sicherer Eingriff und muss von einem erfahrenen Chirurg durchgeführt werden. Es wurden keine prophylaktischen chirurgischen Eingriffe durchgeführt. Phakoemulsifikation ist auch für nanophthalmische und kurzsichtige Augen ein sicherer Eingriff.

Schlüsselwörter: Kataraktoperation, hohe Hypermetropie, Nanophthalmus, Komplikationen.

Key words: Cataract surgery, extreme hypermetropie, nanophthalmus, complications.

P 47. M. Rasp, A. Dexl, M. Emesz, G. Grabner (Salzburg): IntraokularlinsenSubluxation bei Retinitis-Pigmentosa-Patienten - Eine retrospektive Fallserien-Analyse (Poster) - IOL-subluxation in retinitis pigmentosa patients - a retrospective case series analysis

Problemstellung: Im Vergleich zur Normalpopulation entwickeln Patienten mit Retinopathia Pigmentosa (RP) oft schon in relativ jungen Jahren eine Katarakt. Obwohl eine Besserung des für den Patienten am meisten belastenden Symptoms - nämlich des eingeschränkten Gesichtsfeldes - durch die Entfernung der Linsentrübung nicht erreicht werden kann, so ist doch oft eine zumindest subjektive Verbesserung der Sehschärfe, der Blendungsempfindlichkeit und des gestörten Kontrast- und Farbsehens möglich. Bei dieser Patientengruppe muss aber in erhöhtem Ausmaß mit einer Lockerung des Zonularapparates und Schrumpfung des Kapselsackes, samt Dislokation bzw. Subluxation der intraokularen Linse (IOL) gerechnet werden.

Methode und/oder Patienten: Zwischen 1998 und 2009 erfolgte an der Univ.Augenklinik der PMU Salzburg bei vier Augen von zwei RP-Patienten (ein Mann, eine Frau; mittleres Alter 42.5 \pm 12.0 Jahre) eine Entfernung der primär standardmässig „in-the-bag“ implantierten, subluxierten IOL, zeitgleich mit der sekundären Implantation einer Irisklauenlinse. In einer retrospektiven Analyse wurden folgende Befunde ausgewertet: bestkorrigierter Fernvisus, sphärisches Äquivalent, Augendruck, Endothelzellzahl, Details der Photodokumentation, sowie die Erfassung von etwaigen intraoperativen oder postoperativen Komplikationen.

Ergebnisse: Die Zeitdauer zwischen primärer Katarakt-Operation und Zweiteingriff betrug 6,5 \pm 5,3 Jahre. Der nachfolgende mittlere Nachbeobachtungszeitraum betrug 4,3 $\pm 5,3$ Jahre. In drei Augen erfolgte eine Enklavation der IOL vor der Pupille, in einem Auge retro-pupillar. Implantiert wurde in drei Augen eine „AMO VSRA 54“ IOL, sowie in ein Auge eine „Worst Aphakia“ IOL (Ophtec). Die IOL-Stärke betrug +22,8 \pm 2,2 D, die Zielrefraktion betrug -3,0 D, bzw. 0,0 D. Der präoperativ bestkorrigierte Fernvisus stieg von $0,4 \pm 0,2 \mathrm{D}$ auf $0,5 \pm 0,2 \mathrm{D}$, das sphärische Äquivalent postoperativ betrug $-2,3 \pm 2,3 \mathrm{D}$ (Bereich: $-4,2 \mathrm{D}$ bis $0,0 \mathrm{D}$ ). 
Die Endothelzellzahl betrug präoperativ 2966,3 $\pm 226,9$, postoperativ 2129,7 \pm 801,1. An einem Auge wurde eine nach 10 Jahren anterior desenklavierte Irisklauenlinse wiederum problemlos fixiert. Die übrigen Augen waren im postoperativen Verlauf komplikationsfrei.

Schlussfolgerungen: Aufgrund der erhöhten Inzidenz einer Zonula-Schwäche mit konsekutiver deutlicher Kapselsackschrumpfung bei Patienten mit RP sollten regelmäßige Verlaufskontrollen erfolgen, um eine IOL-Dislokation bzw. Subluxation frühzeitig zu erkennen. Der IOL-Tausch mit Implantation einer Irisklauenlinse stellt bei Auftreten des Problems eine exzellente, sichere therapeutische Option dar.

Schlüsselwörter: Retinits pigmentosa, Subluxation, Katarakt, Irisklauenlinse.

Key words: Retinitis pigmentosa, subluxation, cataract, irisclaw-iol.

P 48. O. Findl, N. Hirnschall, S. Tatzreiter, Y. Chen (Wien): Intra-operative Schnittbilddarstellung des vorderen Augenabschnitts mittels OCT bei Kataraktoperation (Poster) - Intraoperative imaging of the anterior eyesegment by OCT during cataract surgery

Problemstellung: Die post-operative axiale Position der Kunstlinse (IOL) verursacht ungefähr zwei Drittel der unbeabsichtigten post-operativen Ametropie. Eine genauere Vorhersage könnte die intra-operative Messung der hinteren Linsekapselposition nach erfolgter Phakoemulsifikation darstellen.

Methode und/oder Patienten: Nach erfolgter Irrigation/Aspiration von Kortexresten und Politur der Kapsel wird die Axiale Position der hinteren Kapsel mittels OCT dargestellt. Diese Position wird mit der postoperativen IOL Position vergleichen welche mittels Laserinterferometrie (AC-Master, Carl Zeiss Meditec) gemessen wird. Zusätzlich werden Beispiele der dynamischen Schnittbilddarstellung des vorderen Augenabschnitts bei der Kataraktoperation gezeigt.

Ergebnisse: Ergebnisse der Messungen der Kapselposition und deren Korrelation zur postoperativen IOL Position werden präsentiert. Zusätzlich werden Beispiele der dynamischen Schnittbilddarstellung des vorderen Augenabschnitts bei der Kataraktoperation gezeigt.

Schlussfolgerungen: Über eine Verbindung zwischen OCT und Operationsmikroskop durch eine Fiberoptik lassen sich intraoperative Abläufe im vorderen Augenabschnitt bei der Kataraktoperation in noch nicht dargestelltem Detail sehen.

Schlüsselwörter: OCT, Katarakt, Biometrie.

Key words: OCT, cataract, biometry.

P 49. M. Georgopoulos, G. Mylonas, S. Schriefel, E. Stifter, R. Menapace (Wien): Vergleich einer angulierten mit einer nicht-angulierten hydrophilen single-piece Acryllinse: 1-Jahresergebnisse (Poster) - One-year results of two single-piece hydrophilic acrylic IOLs with and without haptic angulation

Problemstellung: Für die moderne Mikroinzisionschirurgie werden zunehmend Intraokularlinsen (IOL) aus hydrophilem Acrylmaterial entwickelt. Die neuen Material- und Designfaktoren könnten zu verändertem Kapselsackverhalten und einer höheren Nachstarrate führen. Durch ein anguliertes HaptikDesign ist ein stärkerer Kontakt der Optik mit der hinteren Kapsel zu erwarten und das könnte einen positiven Effekt auf das Nachstarverhalten haben. Dies soll in einem klinischen Vergleich zweier hydrophiler single-piece Acryllinsen mit und ohne Angulierung der Haptiken untersucht werden. Außerdem wird die angulierte IOL in einem „preloaded“ Injektor verwendet. Die Implantationssicherheit wird auch überprüft.

Methode und/oder Patienten: 80 Augen von 40 Patienten mit alterbedingter Katarakt werden in diese bilaterale, prospektive randomisierte Studie inkludiert. Die Implantation der hydrophilen single-piece Acryllinse mit Angulierung der Haptiken (Barrett, Croma) erfolgt mit einem single-use preloaded Injektor nach standardisierter Kleinschnitt-Kataraktchirurgie. Am kontralateralen Auge wird eine vom Design vergleichbare one-piece IOL, jedoch ohne Haptik-Angulierung
Bemerkungen 
implantiert (AcriLyc47LC, Zeiss). Die IOL-Implantation sowie das Kapselsackverhalten und der Nachstar der IOLs werden zu standardisierten Untersuchungszeitpunkten (postoperativ nach 1 Stunde, 1 Woche, 1, 6 und 12 Monate) untersucht (Visante OCT, Retroilluminationsfotografien).

Ergebnisse: Die Verwendung des preloaded-Injektors vereinfacht die Operation und zeigt reproduzierbare IOL-Faltung mit sicherer Kapselsackimplantation. Die Messung der Vorderkammertiefe ergab bei allen Untersuchungszeitpunkten signifikante Unterschiede zwischen beiden IOLs: $3,9 \mathrm{~mm}$ (47LC) vs. $4,3 \mathrm{~mm}$ (Barrett, bedingt durch die Angulierung der Haptiken). Refraktion, Visus sowie Nachstarentwicklung nach einem Jahr zeigten vergleichbare Ergebnisse der beiden Gruppen und werden im Detail präsentiert.

Schlussfolgerungen: Beide IOLs sind durch eine hohe Refraktionsstabilität und niedrige Nachstarentwicklung im ersten postoperativen Jahr gekennzeichnet. Die Barrett-IOL mit dem preloaded-Design zeigt eine reproduzierbare und sichere Implantation.

Schlüsselwörter: Hydrophiles Acrylmaterial, Haptik-Angulierung, preloaded IOL, Nachstar.

Key words: Hydrophilic acrylic material, haptic-angulation, preloaded IOL, PCO.

P 50. M. Rau (Cham): Erste Ergebnisse mit der Oculentis LENTIS Mplus IOL (Poster) - The first results with the Oculentis LENTIS Mplus IOL

Problemstellung: Bis jetzt verfügten alle multifokalen Linsen, diffraktiv wie auch refraktiv, über rotationssymetrische Konzepte mit konzentrischen Ringen. Die Probleme Zweck dieser Studie war die Beurteilung der optischen, funktionellen Ergebnisse, wie Kontrastempfindlichkeit und Patientenzufriedenheit nach Implantation der multifokalen IOL LENTIS ${ }^{\circledR}$ Mplus (Oculentis), die einen völlig neuen Ansatz in der multifokale Linsentechnologie verfügt. Sie kombiniert eine asphärischen asymmetrischen Fernbereich mit einem nahtlosen Übergang zum sektorförmigen Nahbereich mit einer Addition von +3,0 Dioptrien. Die LENTIS $^{\circledR}$ Mplus ist pupillenunabhängig und basiert auf ein refraktives Prinzip.

Methode und/oder Patienten: In einer prospektiven Fallstudie, von September 2009 bis Dezember 2009 haben wir die LENTIS ${ }^{\circledR}$ Mplus in 24 Augen von 16 Patienten implantiert. Wir untersuchten die postoperativen Refraktion, unkorrigiert (UCVA) und bestkorrigiert (BCVA), Fern-, Intermidiär- und Nahsehschärfe, Lesegeschwindigkeit mit Radner Charts und die Kontrastempfindlichkeit 3 und 6 Monate nach der Implantation. Die Qualität des Sehens, Blendung, Halos und die persönliche Zufriedenheit der Patienten wurde mit einem validierten Fragebogen bewertet.

Ergebnisse: 6 Monate nach der Implantation war der mittlere Fernvisus UCVA 0,75 und für die Nähe 0,8. Die Kontrastempfindlichkeit untersucht mit Pelli RobsonCharts war 1,67. Die durchschnittliche Kontrastempfindlichkeitskurve FACT (Ginsburg) lag im normalen Bereich. Nur $24 \%$ und $10 \%$ der Patienten beschrieben auf Nachfrage Halos und Blendung zu sehen. Alle Patienten waren mit dem Ergebnis zufrieden.

Schlussfolgerungen: Die Ergebnisse nach Implantation von LENTIS ${ }^{\circledR}$ Mplus sind vielversprechend, zeigen Verbesserung der Kontrastempfindlichkeit und reduzieren nachweislich Halos und Glare.

Schlüsselwörter: Grauer Star, Alterssichtigkeit, Multifokallinsen, LENTIS Mplus. Key words: Cataract, presbyopia, multifocal lenses, LENTIS Mplus.

P 51. S. Pieh, W. Fiala (Wien): Auswahl eines aspährischen Intraokularlinsendesigns anhand kornealer Parameter (Poster) - Choosing aspherical IOL design using corneal parameters

Problemstellung: Aberrationskorrigierende IOLs verbessern die Abbildungsqualität des Auges nach einer Kataraktoperation, bei entsprechender Korrektur der tatsächlich bestehenden kornealen sphärischen Aberration, sowie einer ausreichenden Zentrierung des Implantates. 
Methode und/oder Patienten: Anhand von theoretischen Berechnungen sowie Laboruntersuchungen wird bei Hornhäuten mit einer niedrigen, mittleren und hohen sphärischen Aberration die Abbildungsqualität aberrationsfreier und unterschiedlicher aberrationskorrigierender IOL in Abhängigkeit von der IOL Dezentrierung untersucht.

Ergebnisse: Mit zunehmender Dezentrierung zeigen aberrationsneutrale Vorteile gegenüber aberrationskorrigierenden IOLs.

Schlussfolgerungen: Die gemessene sphärische kornealer Aberration der individuellen Hornhaut sollte immer unterkorrigiert werden. Ohne Kenntnisse der Hornhautparameter sind aberrationsfreie IOLs die sicherste Wahl.

Schlüsselwörter: IOL, korneale sphärische Aberrationen, asphärisches IOL design.

Key words: IOL, corneal spherical aberration, aspheric lens design.

P 52. S. Wanderer, N. Hirnschall, S. Tatzreiter, O. Findl (Wien): Vergleich von zwei Purkinjemetern zur Messung der Verkippung und Dezentrierung von Intraokularlinsen (Poster) - Comparison of two purkinjemeters to measure tilt and decentration of intraocular lenses

Problemstellung: Die Messung der Purkinjereflexe ermöglicht eine Quantifizierung der Verkippung und Dezentrierung von Intraokularlinsen (IOL). Die dadurch beschriebene Lage ist wichtig für die resultierende optische Abbildungsqualität, vor allem bei asphärischen und mulitfokalen IOL. Derzeit gibt es zwei Purkinjemeter Systeme mit publizierten Daten bei pseudophaken Augen mit modernen IOL, das System von Artal und Kollegen (Murcia, Spanien) und Schaeffel (Tübingen, Deutschland). Ziel dieser Studie ist der direkte Vergleich dieser zwei Messysteme und die Anwendbarkeit im klinischen Alltag zu untersuchen.

Methode und/oder Patienten: Im Rahmen dieser Vergleichsstudie wurde bei pseudophaken Patienten in Mydriasis die Dezentrierung und Verkippung der IOL vermessen. Um die IOL Position zusätzlich mit anderen Methoden zu dokumentieren wurden auch standardisierte Retroilluminationsfotos und Scheimpflugaufnahmen (Galilei, Ziemer, Schweiz)durchgeführt.

Ergebnisse: Beide Geräte zeigten sich nach bisheriger Erfahrung in der klinischen Anwendbarkeit bei älteren pseudophaken Patienten als anwendbar. Geringe bis mittelgradige Ausmasse von Verkippung und Dezentrierung sind gut messbar. Extremwerte bei deutlich verkippten und dezentrierten Fällen waren mit beiden Geräten schwer messbar.

Schlussfolgerungen: Die Quantifizierung von IOL Dezentrierung und Verkippung ist für die Entwicklung von Haptikdesigns von IOL von großer Bedeutung. Schlüsselwörter: Purkinjemeter, Purkinjereflexe, IOL Dezentrierung, IOL Verkippung.

Key words: Purkinjemeter, purkinje reflexes, intraocular lens tilt, intraocular lens decentration.

P 53. S. Pieh, G. Schmidinger, C. Skorpik (Wien): Abbildungsqualität der OptiVis II in klinischen Untersuchungen (Poster) - Image quality of the OptiVis II in clinical examinations

Problemstellung: Ein neues multifokales IOL Design wird einem klassischen diffraktiven Design mit Betonung des Fernbrennpunktes in klinischen Untersuchungen gegenübergestellt.

Methode und/oder Patienten: Bei 20 Augen von 15 Patienten (Alter 67,5 Jahre \pm 13,1) mit einer OptiVis IOL und bei 20 Augen von 14 Patienten (Alter 67,1 Jahre \pm 12,4) mit einer Acri.Lisa wurden Defokusierungskurven in 0,25 Dioptrien Schritten mit bester Fernkorrektur durchgeführt, die beste subjektive Lesedistanz mit bester Fernkorrektur ermittelt, die größte Pupillenweite unter mesopischen Bedingungen (Infrarotkamera, Keratograph, Fa. Occulus) und die Fernkontrastsehschärfe mit bester Korrektur (Low Visual Acuity Charts 10\%, Precision Vision) festgestellt.
Bemerkungen 
Ergebnisse: Beide Linsen erreichen mit bester Fernkorrektur eine vergleichbare Sehschärfe von logMar 0,0 \pm 0,12 für die OptiVis und logMar 0,03 \pm 0,07 für die Acri.Lisa. Die beste Nahsehschärfe von logMar 0,19 erreichen die Patienten mit der OptiVis bei einer Defokusierung von $-2,5$ Dioptrien, bei der Acri.Lisa eine Nahsehschärfe von logMar 0,17 \pm 0,09 bei einer Defokusierung von -3,0 Dioptrien. Subjektiv wählten die Patienten mit der OptiVis einen Leseabstand von $36,0 \mathrm{~cm} \pm 2,33$. Bei der Acri.Lisa 32,60 $\mathrm{cm} \pm 2,07$. Die Pupillenweite in der OptiVisGruppe betrug unter mesopischen Bedingungen 4,47 $\mathrm{mm} \pm 0,8$, in der Acri.LisaGruppe 4,82 mm $\pm 0,76$. Die Untersuchung mit den Low Visual Acuity Charts $10 \%$ ergab für die OptiVis-Gruppe einen Wert von logMar 0,17 $\pm 0,12$ und für die Acri. Lisa-Gruppe von logMar 0,14 $\pm 0,15$.

Schlussfolgerungen: Die beiden untersuchten Linsen unterschieden sich bei den hier durchgeführten Untersuchungen vor allem durch eine unterschiedliche Nahaddition.

Schlüsselwörter: IOL, diffraktive Bifokallinse, intraokulare Multifokallinse, Defokussierungskurve.

Key words: IOL, diffractive bifocal IOL, intraocular multifocal IOL, defocus curves.

P 54. E. Smretschnig, J. Spörl, S. Binder, U. Stolba (Wien): Lebensstil und präsenile Katarakt: kausale Zusammenhänge bei Unter-60-jährigen (Poster) Lifestyle and pre-senile cataract: causal associations in patients under 60 years

Problemstellung: Es existieren bereits zahlreiche Publikationen die sich mit den Risikofaktoren der Katarakt-Enstehung im Generellen befasst haben, unsere Studie hat gezielt die Umwelteinflüsse und den Lebensstil der Unter-60-jährigen untersucht. Ziel dieser analysierenden, retrospektiven Studie war es, die Exposition exogener Noxen von Katarakt operierten Patienten unter 60 Jahren zu erfassen, statistisch auszuwerten und mit der bestehenden Literatur zu vergleichen.

Methode und/oder Patienten: In dieser retrospektiven Studie wurden die demographischen Daten von 100 konsekutiven Patienten die zum Zeitpunkt ihrer Katarakt Operation in der Krankenanstalt Rudolfstiftung das 60. Lebensjahr noch nicht vollendet hatten, mittels eines Fragebogen erhoben und auf statistische Signifikanzen untersucht. Die Fragen nach dem Lebensstil umfassten den Wohn- und Geburtsort, Berufsleben, BMI, Nikotinabusus sowie ophthalmologische und systemische Komorbiditäten und damit verbundene medikamentöse Therapien. Traumatische Katarakte wurden ebenso aus der Statistik ausgeschlossen wie Katarakt Operationen im Rahmen eines kombiniert-chirurgischen Eingriffs.

Ergebnisse: In dem befragten Patientengut fand sich Nikotinabusus überproportional oft, dabei war die nukleäre Katarakt die am häufigsten vertretende Kataraktform, das chronische Glaukom die am meisten vertretene ophthalmologische Nebendiagnose. Hinsichtlich Adipositas und Nikotinabusus fanden sich keine geschlechtsspezifischen Unterschiede. Weiters bestanden beim untersuchten Patientengut oft Diabetes und Hypertonie als Systemerkrankung.

Schlussfolgerungen: Die Prävalenz des Nikotinabusus und das Auftreten einer prä-senilen Katarakt stehen in einem kausalen Zusammenhang miteinander.

Schlüsselwörter: Katarakt, Umwelteinfluss, Lebensstil, Nikotin.

Key words: Cataract, enviromental factor, lifestyle, nicotine.

P 55. N. Weber, S. Mennel, W. Sekundo (Marburg): Zusammenhang zwischen dem Durchmesser der Optik und der A-Konstante am Beispiel der Intraokularlinse Model 620H (Poster) - Does the size of IOL's affect the A-constant?

Problemstellung: Die vom Hersteller angegebene A-Konstante für die IOL $620 \mathrm{H}$ (Superflex, Rayner LTd/GB) ist ein objektiv ermittelter Wert, der sich von dem Wert des Models 570C (C-Flex, Rayner Ltd./GB) ableitet. Der Unterschied zwischen den beiden Modellen liegt im Durchmesser (Superflex: 6,25 mm, C-Flex: 
5,75 mm). Ziel dieser Studie war zu evaluieren, ob die Änderung des OptikDurchmessers eine Anpassung der A-Konstante erfordert.

Methode und/oder Patienten: Es wurde an 48 Augen von 41 Patientin zwischen 10/2005 und 08/2006 durch einen erfahrenen Chirurgen in clear cornea Technik eine Phakoemulsifikation mit Hinterkammerlinsen-Implantation durchgeführt. Zur IOL-Berechnung wurde die optische Kohärenzbiometrie (IOL-Master, Carl Zeiss Meditec)und die SRK/T-Formel verwendet. Drei Monate postoperativ wurde die objektive und subjektive Refraktion der Patienten erhoben, in das Berechnungsprogramm des IOL-Masters eingegeben und die A-Konstante optimiert. Mit der modifizierten A-Konstante wurden 20 Augen von 20 Patienten auf dieselbe Art und von demselben Chirurgen operiert und nachbeobachtet.

Ergebnisse: Die vom Hersteller empfohlene A-Konstante für die optische Kohärenzbiometrie und die SRK/T-Formel von 119,25 wurde auf 118,90 optimiert. Das entspricht einer IOL-Stärken-Differenz von 0,5 dpt.

Schlussfolgerungen: Die Änderung des Optik-Durchmessers führt trotz gleicher IOL-Geometrie zu einer Änderung der A-Konstante. Wir empfehlen die neu ermittelte A-Konstante von 118.9 für die SRK/T-Formel und das Model 620H an eigenen Patienten zu überprüfen.

Schlüsselwörter: A-Konstante, IOL-Master, IOL-Design.

Key words: A-Constant, IOL-Master, IOL-Design.

P 56. S. Tatzreiter, N. Hirnschall, M. Rossmann, Y. Chen, T. Neumayer, O. Findl (Wien): Morphologische Veränderungen von regeneratorischem Nachstar nach modifizierten Nd:YAG Laser-Verfahren. (Poster) - Morphological changes in regeneratory posterior capsule opacification after modified $\mathrm{Nd}$ :YAG laser-treatments

Problemstellung: Beschreibung der morphologischen Veränderungen von Elschnig-Perlen nach modifizierten Nd:YAG Laser-Verfahren.

Methode und/oder Patienten: 27 Patienten mit Nachstar und Indikation zur Nd:YAG-Laser-Kaspulotomie wurden eingeschlossen. In der ersten Kohorte wurde eine kleinstmögliche Öffnung der hinteren Kapsel außerhalb der Sehachse mittels Nd:YAG Laser durchgeführt (,MiniYAG') und in der zweiten Kohorte wurde niedrigdosiert $(0,3 \mathrm{~mJ})$ behandelt, ohne die hintere Kapsel zu eröffnen (,GentleYAG'). In beiden Kohorten wurden vier Quadranten der hinteren Kapsel innerhalb der Rhexis definiert und der zu behandelnde Quadrant randomisiert. Veränderungen des Nachstars wurden subjektiv (PCO Score: 0-10) und objektiv (AQUA Score: 0-10) von Retroilluminationsfotos beurteilt.

Ergebnisse: 17 Augen wurden in der MiniYAG Kohorte und 10 Augen in der GentleYAG Kohorte rekrutiert. Das PCO-Grading wurde nach MiniYAG signifikant ( $p>0,01$ ) von 5,2 (SD: 1,6) auf 4,0 (SD: 1,9) verringert. In der GentleYAG Kohorte wurde nur bei einem Patienten eine Verringerung des Nachstars beobachtet und die Nachstarreduktion war nicht signifikant (AQUA Score Reduktion um 0,1, SD: $1,2 ; \mathrm{p}=0,8)$.

Schlussfolgerungen: Die Ergebnisse deuten darauf hin, dass die Laser-Schockwelle gemeinsam mit dem Eindringen von Flüssigkeit in den Linsen-Kapsel Zwischenraum für die morphologischen Veränderungen der Elschnig Perlen verantwortlich ist.

Schlüsselwörter: Nachstar, Elschnig-Perlen, Nd:YAG Laser Kapsulotomie, Laser Schockwelle, niedrigdosierter Laser.

Key words: After star, posterior capsule opacification, Elschnig pearls, YAG laser capsulotomy, laser shockwave, low dose laser.
Bemerkungen 
P 57. Y. Chen, N. Hirnschall, O. Findl (Wien): Tränenfilmersatz während der Kataraktoperation mittels eines viskösen Tränenersatzmittels (Poster) Corneal wetting with a viscous eye lubricant to maintain optical clarity during cataract surgery

Problemstellung: Vergleich der Benetzungseigenschaften von Balanced salt solution (BSS) und eines viskösen Tränenersatzmittels während der Kataraktoperation.

Methode und/oder Patienten: In dieser randomisierten, kontrollierten, doppelblinden Studie wurden Patienten mit einer geplanten Kataraktoperation inkludiert. Die Patienten erhielten randomisiert entweder BSS oder Cornea_Protect, ein 2\%iges Hydroxypropylmethylcellulose (HPMC) Gel. Beurteilt wurde die Anfärbung der Hornhaut mit Fluoreszein (Grad 0-12), der Grad der intraoperativen Hornhautklarheit (1-5), die Applikationshäufigkeit, die subjektive Einschätzung des Fremdkörpergefühls und des Trockenheitsgefühls des Auges durch den Patienten mittels einer visuellen Analogskala.

Ergebnisse: Einhundertundein Augen von 97 Patienten (mittleres Alter 73,6 Jahre; von 53 bis 87 Jahre) wurden in diese Studie inkludiert. Mittlerer Grad der Fluoreszeinfärbung war 9,46 (SD: 3,64) in der BSS-Gruppe und 9,76 (SD: 3,27) in der $2 \%$ HPMC-Gruppe ( $p=0,67)$. Es zeigte sich eine statistisch signifikante Differenz $(p=0,03)$ zwischen dem Median der intraoperativen Hornhautklarheit der BSS- $(2,0)$ und der 2\% HPMC-Gruppe $(1,0)$. Die mediane Applikationshäufigkeit von BSS war zehnmal so hoch $(10,0)$ im Vergleich zu $2 \%$ HPMC $(1,0 ; p<0,001)$.

Schlussfolgerungen: Der Einsatz von 2\% HPMC führt im Vergleich zu BSS zu einer höhergradigen Klarheit der Hornhaut während der Kataraktoperation. Eine einmalige Applikation von 2\% HPMC zu Beginn der Operation ist ausreichend um eine gute Hornhautklarheit zu gewährleisten. Dies führt zu einem besseren Einblick für den Operateur. Da keine Notwendigkeit für regelmäßiges Spülen besteht führt die Anwendung von 2\% HPMC außerdem zu einem höheren Komfort für den Patienten in Lokalanästhesie.

Schlüsselwörter: Hydroxpropylmethylcellulose, HPMC, BSS, Tränenfilmersatz, Hornhautbenetzung.

Key words: Hydroxpropylmethylcellulose, HPMC, balanced salt solution, BSS, corneal lubricant, corneal wetting agents.

\section{QUALITÄT, GLAUKOM, EXPER. OPHTHALMOLOGIE}

P 58. C. Wollinger, N. Hirnschall, R. Paradies, C. Flakowski, G. Schulte, O. Findl (Wien): Computergestütztes Informationsprogramm als unterstützendes Mittel bei der Verbesserung der Qualität und Effizienz des Einwilligungsprozesses bei der Kataraktoperation (Poster) - Computer-based tutorial as supportive means to enhance quality and efficiency of the informed consent process for cataract surgery

Problemstellung: Voraussetzung für eine informierte Entscheidung zu einer Operation setzt bei der Patientenseite ein Verständnis über die Erkrankung und den geplanten Eingriff sowie die Risiken voraus. Aus der Literatur geht hervor, dass Patienten, die vor einer Kataraktoperation stehen oftmals die Zusammenhänge schlecht verstehen und sich Inhalte aus dem Aufklärungsgespräch schlecht merken. Aufgrund der asymmetrischen Arzt-Patienten-Beziehung wird bei Unklarheiten während des Aufklärungsgespräches oftmals nicht nachgefragt. Um das Verständnis und die Merkfähigkeit zu verbessern soll in dieser Studie ein computergestütztes Informationsprogramm evaluiert werden.

Methode und/oder Patienten: Das von uns entwickelte Cat-Info Programm stellt in einfacher Form mit Hilfe von Diagrammen, Animationen und Bildern die nötigen Informationen über die Katarakt, deren Behandlung und Therapieverlauf dar. Über Kopfhörer wird den Patienten die Information auch wiedergegeben. Nach jedem Kapitel müssen die Patienten über einen Touchscreen durch Berühren einer Ampel ihr Feedback geben. Das Design der Studie ist randomisiert, kontrolliert, Patienten- und Untersucher maskiert. 90 Patienten wurden einge-
Bemerkungen 
schlossen, 60 davon in der Studiengruppe, 30 in der Kontrollgruppe. Letztere erhielt eine kurze Computerpräsentation ohne spezifische Informationen zur Katarakt, die Studiengruppe dagegen das beschriebene Cat-Info Programm. Im Anschluss an die Präsentation erhielt jeder Patient das übliche persönliche ärztliche Aufklärungsgespräch und beantwortete einen Fragebogen.

Ergebnisse: Es wurde ein Fragebogen validiert. Die Ergebnisse des Wissenstands und auch der subjektiven Einschätzung der Patienten bezüglich des Aufklärungstools werden präsentiert.

Schlussfolgerungen: Das Ziel der Studie ist, die Aufklärung zur Kataraktoperation durch das computergestützte Informationsprogramm zu optimieren, um das persönliche Arzt-Patienten-Gespräch zielgerichteter und zeiteffizienter führen zu können. Aus rechtlichen Gründen kann das ärztliche Aufklärungsgespräch dadurch aber nicht ersetzt werden.

Schlüsselwörter: Katarakt, Aufklärung, computergestütztes Informationsprogramm.

Key words: Informed consent, computer-based tutorial, cat-info.

P 59. S. Huber, C. Zehetner, E. Schmid, N. Bechrakis (Innsbruck): Auswertung der Notfallambulanz der Universitätsklinik für Augenheilkunde und Optometrie, Innsbruck 2010 (Poster) - Analysis of the emergency patients 2010 at the Department of Ophthalmology, Medical University of Innsbruck, Austria

Problemstellung: Seit ca. zwei Jahren beobachten wir ein vermehrtes Aufkommen von Notfall-Patienten, sowohl unter der Woche als auch am Wochenende. Bisher stand uns keine einfach praktizierbare Möglichkeit zur Verfügung, die in der Notfallambulanz erhobenen Daten auszuwerten. Seit Mitte November 2009 ist die Dokumentation in der Notfallambulanz der Universitätsklinik für Augenheilkunde und Optometrie in Innsbruck auf eine papierlose elektronische Dokumentation umgestellt. Daraus ergibt sich jetzt erstmals die Möglichkeit die dadurch generierten Daten einfach statistisch auszuwerten.

Methode und/oder Patienten: Wir verwenden die Daten des Kalenderjahrs 2010. Es werden alle Patienten, die im Jahr 2010 die Notfallambulanz der Universitätsklinik für Augenheilkunde und Optometrie aufgesucht haben anonym ausgewertet. Der Datensatz, der über 9000 Patientenkontakte enthält, wird bzgl. häufigster Diagnosen, der Geschlechtsaufteilung, des tageszeitlichen Verlaufs, der Wochenverteilung und der Wartezeiten ausgewertet.

Ergebnisse: Häufigste Diagnosen waren Hornhaut-Fremdkörper, konj. Fremdkörper, virale Konjunktivitis, Hordeolum, bakt. Konjunktivitis, allergische Konjunktivitis, Hyposphagma, Keratitis, Keratokonjunktivitis. Die Geschlechtsaufteilung zeigt einen Überhang an männlichen Patienten. Der tageszeitliche Verlauf zeigt einen steilen Anstieg der Frequenz am Vormittag mit einer kleinen „Mittagsdelle“, um am frühen Nachmittag anzusteigen und gegen 16h abzufallen. Ein neuerlicher Gipfel wird gegen 17h/18h erreicht. Die Wochenverteilung zeigt das höchste Patientenaufkommen am Freitag gefolgt vom Montag. Der am dritthäufigsten frequentierte Tag ist der Samstag. Die Wartezeit beträgt im Durchschnitt 83 min. Zusätzlich zeigt sich, dass ein erheblicher Teil (bis zu 1/3) der Patienten auf Tagesrandzeiten bzw. aufs Wochenende ausweicht. Der Samstag, bringt ein deutlich höheres Aufkommen als der Sonntag.

Schlussfolgerungen: Mit über 9000 Notfallpatienten im Jahr 2010 stellt der Notfallpatient einen großen Teil der Klientel der Augenklinik dar und ist dadurch eine große Herausforderung für den klinischen Routinebetrieb. Da die häufigsten 10 Diagnosen Augenerkrankungen betreffen, die problemlos von einem niedergelassenen Augenfacharzt behandelt werden könnten, stellen diese Patienten eine Belastung der Kapazitäten einer Universitätsklinik dar. Eine Rückführung der Patientenströme in den niedergelassenen Bereich ist eine der Herausforderungen für die Zukunft, da durch finanzielle und personelle Ressourcenverknappung solche Leistungen nicht mehr in diesem Umfang an einer Klinik erbracht werden können.
Bemerkungen 
Schlüsselwörter: Notfallambulanz, Auswertung.

Key words: Emergency, analysis.

P 60. A. Wedrich, G. Langmann, A. Langmann, C. Wohlfahrt, M. Weger, E. Granitz, A. Kohlhof (Graz): Entwicklung und Implementierung eines Leitbildes an der Universitäts-Augenklinik Graz (Poster) - Development and implementation of a mission statement at the University Department of Ophthalmology Graz

Problemstellung: Die Entwicklung eines Leitbildes - bestehend aus Vision, Mission und Werten - als Basis für eine von allen Berufsgruppen einer Universitätsklinik getragenen Strategie und Weiterentwicklung der Organisation ist aufgrund der vielfältigen Aufgaben eine besondere Herausforderung. Ein gemeinsam erarbeitetes Leitbild ermöglicht die Fokussierung aller MitarbeiterInnen auf ein gemeinsames Ziel

Methode und/oder Patienten: Als Ziel unseres EFQM Projektes wurde die gemeinsame Entwicklung eines Leitbildes bestehend aus einer Vision, Mission und Werten definiert. Leitende aller an unserer Klinik vertretenen Berufsgruppen wurden an der Teilnahme eingeladen. Nach Erarbeitung des Leitbildes in monatlichen Sitzungen unter Einbezug von Feedback-Schleifen und externer Begleitung wurde eine dreimonatige Phase der Kommunikation in allen Berufsgruppensitzungen mit intensiver Diskussion der Inhalte des Leitbildes durchgeführt. Email-Aussendungen, graphisch aufbereitete Unterlagen, Poster, Bildschirmschoner, und MitarbeiterInnengespräche unterstützten die Kommunikation des Leitbildes. Für den positiven Projektabschluss mussten $80 \%$ der TeilnehmerInnen einer Klinikbefragung die wesentlichen Inhalte des Leitbildes wiedererkennen.

Ergebnisse: Durch die Feedback-Schleifen zwischen den Sitzungen konnte in 5 Sitzungen von den Leitenden aller Berufsgruppen eine Vision, Mission und Werte entwickelt werden. In weiteren drei Sitzungen wurde eine graphische Aufbereitung der Leitbildsätze abgeschlossen und ein Fragebogen erarbeitet. Die Befragung wurde mit 40\% der randomisiert ausgewählten Angehörigen aller Berufsgruppen gemeinsam im Hörsaal der Klinik durchgeführt und ergab, dass in 98\% die Inhalte des Leitbildes wieder erkannt wurden. Die Zustimmung zu den Inhalten des Leitbildes betrug über $80 \%$.

Schlussfolgerungen: Unter Einbeziehung aller Berufsgruppen war es möglich, im Rahmen des EFQM Modells unter Einbeziehung der RADAR Logik (kontinuierliche Feedbackschleifen) ein Leitbild für die Univ. Augenklinik zu erarbeiten und zu kommunizieren, das von über $80 \%$ einer repräsentativen Gruppe der Klinik akzeptiert wurde.

Schlüsselwörter: Qualitätsmanagement, Leitbild, Vision, Mission, UniversitätsAugenklinik, EFQM-Modell.

Key words: Quality management, mission statement, University Department of Ophthalmology, EFQM.

P 61. V. Schöllbauer, C. Brennig, E. Walter (Wien): Kosten der Blindheit in Österreich (Poster) - Costs of blindness in Austria

Problemstellung: Literatur zur Epidemiologie der Blindheit und die mit diesem Krankheitsbild verbundenen ökonomischen Auswirkungen sind sehr rar. Das Ziel der vorliegenden Studie ist es, diese Forschungslücke für Österreich zu schließen und die gesamten wirtschaftlichen Auswirkungen der Blindheit für die österreichische Gesellschaft zu evaluieren.

Methode und/oder Patienten: Für die vorliegende Krankheitskostenstudie wurde der Inzidenzansatz gewählt. Die Berechnungen erfolgten für alle Ursachen von Blindheit, darunter zählen zu den häufigsten AMD, Glaukom, diabetische Retinopathie Katarakt sowie andere Ursachen. Ebenso erfolgte eine Unterteilung der Krankheitsbilder in Altersgruppen. Die Krankheitskosten umfassen direkte medizinische Kosten welche unmittelbar (durch die Behandlung, Arztkonsultatio- 
nen, Hilfsmittel, Depressionskosten, Hüftfrakturen etc.) entstehen und direkte nicht-medizinische Kosten (Adaptierung der Wohnung), die sich durch die Folgen der Erkrankung ergeben. Das Pflegegeld und Kosten für Betreutes Wohnen fanden als indirekte Kosten Berücksichtigung. Die Analyse wird aus der Perspektive der Gesellschaft durchgeführt. Die Kosten wurden vom Zeitpunkt der Erblindung über das reduzierte Restleben der Patienten berechnet (Diskontsatz 5\%).

Ergebnisse: In Österreich erblinden jährlich 15.734 (Inzidenz, nach Trautner et al.) Personen. Die größte Patientengruppe ist jene der $\geq 80$-jährigen mit 13.299 Menschen. Die Gesamtkosten (direkte und indirekte Kosten) der Blindheit über alle Altersgruppen inkl. der Transferkosten (Pflegegeld) belaufen sich auf $€ 516.155 .507$ bzw. auf $€$ 132.096.542,97 (diskontiert) für die Restlebenszeit der Patienten, wobei die direkten Kosten $€$ 7.452.575,84 (diskontiert) und die indirekten Kosten 124.643.967,13 EUR (diskontiert) ausmachen. Die Gesamtkosten exkl. der Transferkosten der Blindheit belaufen sich auf $€ 344.311 .138,85$ bzw. auf $€$ 88.117.457,81 (diskontiert) (entspricht 6\% direkten und 94\% indirekten Kosten). Die höchsten indirekten Kosten werden in der Patientengruppe „Anderes“ von den 18-59-jährigen, gefolgt von Opticusatrophie, Diabetischer Retinopathie bzw. AMD in der Altersgruppe 60-79 Jahre verursacht.

Schlussfolgerungen: Aufgrund des demographischen Wandels ist mit einer Zunahme der Anzahl älterer Menschen in den nächsten Jahren zu rechnen. Eine Vielzahl der Ursachen von Blindheit trifft primär ältere Menschen (AMD, diabetische Retinopathie). Die demographische Entwicklung wird zu einer steigenden Zahl von blinden Personen führen und somit zu einem kostspieligen Public Health Problem. Mit dem Verlust der Sehkraft sind nicht nur enorme Kosten verbunden, sondern auch die Lebensqualität der Betroffenen wird dadurch maßgeblich beeinflusst. Die Analyse zeigt, dass Blindheit hohe Kosten verursacht und innovative Behandlungsalternativen zur zukünftigen Kosteneinsparung betragen könnten.

Schlüsselwörter: Blindheit, direkte Kosten, indirekte Kosten.

Key words: Blindness, direct costs, indirect costs.

P 62. C. Wohlfart, J. Wagner, R. Maier, G. Langmann, A. Wedrich, H. Bauer, U. Klug (Graz): EFQM Projekt der Univ.-Augenklinik im Rahmen der C2E Rezertifizierung Management ambulanter PatientInnen ohne Termin (Poster) - EFQM Recertification at Department of Ophthalmology, Medical University Graz Management of outpatients without appointment

Problemstellung: Zu den Kernaufgaben der Ambulanz der Univ.-Augenklinik Graz gehören laut Krankenanstaltengesetz terminisierte postoperative Kontrollen, Spezialuntersuchungen sowie Notfälle. Alle anderen PatientInnen ohne Termin werden zu den niedergelassenen FachärztInnen verwiesen (= Triagegespräch). Dies fand bis 2009 in unserer Ambulanz unmittelbar neben den Augenuntersuchungen statt und belastete das Ambulanzteam durch vermehrten Zeitaufwand, Unverständnis der PatientInnen und häufige Unterbrechungen der Augenuntersuchung. Es gab keinen transparenten Prozess für die Triage, keine nachvollziehbare Dokumentation der Triagegespräche und damit keine Grundlage für ein strukturiertes Beschwerdemanagement.

Methode und/oder Patienten: Ziel des Projekts und Vorgabe der Anstaltsleitung war die Reduktion der Anzahl ambulanter PatientInnen ohne Termin. Folgende Maßnahmen wurden gesetzt: 1 . Zusammenstellen eines interdisziplinären Teams 2. Evaluation der Anzahl von PatientInnen ohne Termin 3. Prozessdefinition und Erarbeiten von Verbesserungspotentialen 4. Adaptation des Prozesses mit Leistungserfassung im Medocs 5. Implementierung eines Informationspakets über unser Triagesystem für alle Interessensgruppen: ambulante PatientInnen (hausinterne Poster und Beschilderung), alle MitarbeiterInnen der Augenklinik (Rundschreiben), Verwaltung (Checkliste), externe FachärztInnen (Rundschreiben), HausärztInnen (Ärztekammerzeitung), steirische Kliniken hausintern und -extern (Rundschreiben), Bevölkerung (Klinik-Homepage und Kleine Zeitung). 6. Im Oktober 2010 fand nach Implementierung der Maßnahmen eine Reevaluation statt.

Ergebnisse: Die Evaluation 2009 ergab durchschnittlich 15 Triagegespräche pro 
Tag. Diese Zahl konnte durch das Maßnahmenpaket während eines Jahres deutlich reduziert werden: 2010 wurden um 30\% weniger Triagegespräche registriert. Zusätzlich konnte ein für alle Berufsgruppen verbesserter Ablauf in die Routine eingeführt und dokumentiert werden.

Schlussfolgerungen: Mittels genauer Prozessanalyse und Implementierung eines strukturierten Infopakets durch das interdisziplinäre Ambulanz-Team wurde innerhalb eines Jahres eine Reduktion der Triagegespräche um 30\% erreicht. Schlüsselwörter: EFQM, Ambulanzfrequenz, Qualitätsmanagement, Triage.

Key words: Quality management, frequencies of outpatient clinic, triage.

P 63. C. Mayer, M. Lackner, M. Brandner, S. Lindner, G. Langmann, A. Wedrich, A. Langmann (Graz): Erstellen eines Ausbildungscurriculms für AssistentInnen in Facharztausbildung im Teilbereich Strabologie - European Foundation for Quality Management (EFQM) Projekt (Poster) - Developing a residency training in strabismus - European Foundation for Quality Management (EFQM) Project

Problemstellung: Derzeit existiert an der Augenklinik Graz kein einheitliches Ausbildungscurriculum für AssistenInnen. Das Erstellen eines Curriculums auf internationalem Niveau im Rahmen des EFQM Projektes - Committed to Excellence (C2E) wurde als Ziel definiert. Die derzeitige Ausbildung an der Grazer Augenklinik setzt sich aus einem Rotationssystem durch die verschiedenen Stationen und Ambulanzen zusammen. Die Lernziele richten sich nach dem Rasterzeugnis der Österreichischen Ophthalmologischen Gesellschaft. 6 Monate dieser Ausbildungszeit sind in der Ambulanz für Schielen, Kinderophthalmologie und Rehabilitation Sehbehinderter zu absolvieren.

Methode und/oder Patienten: Als Ziel des Projektes wurde das Erstellen eines Konzeptes für die Ausbildung von AssistentInnen auf internationalem Niveau im Bereich Strabologie definiert. Der Ablauf des Curriculums wurde in 3 Phasen eingeteilt. Die dreitägige Einführungsphase beinhaltet eine Einführung in die orthoptischen Untersuchungsmethoden, Zuteilung der TutorInnen, die Einführungsmappe, Rasterzeugnis sowie die Basisliteraturmappe Strabologie. In der sechsmonatigen Umsetzungsphase sind konkrete Falluntersuchungen durchzuführen und es wird eine wöchentliche Logbookstunde (European Board of Ophthalmology und den ICO Richtlinien) angeboten. Auch das wissenschaftliche Arbeiten im Bereich Strabologie soll während dieser Phase ein Teil der Ausbildung sein. Der strukturierte Evaluierungsprozess findet als Abschluss des Curriculums statt (Selbst- und Fremdreflexion sowie Evaluierung der Kenntnisse und Fertigkeiten).

Ergebnisse: Als Messkriterien wurden ein vorliegendes Konzept sowie die Vorstellung des Pilotprojektes im Rahmen einer Informationsveranstaltung festgelegt. Die Veranstaltung sollte für das gesamte Klinikpersonal mit mindestens 70 prozentiger Anwesenheit des ärztlichen und orthoptischen Personals abgehalten werden.

Schlussfolgerungen: Nach einer 6-monatigen Projektarbeit zur Erlangung der Committed to Excellence (C2E) Auszeichnung wurde ein detaillierter Lernzielkatalog erarbeitet, der sowohl den Anforderungen der EBO (European Board of Ophthalmology) als auch ICO (International Council of Ophthalmology) entspricht. Der/die Assistent/in erhält mit diesem Curriculum die theoretischen und praktischen Fertigkeiten, die ihn befähigen, die Prüfung zum Europäischen Facharzt für Augenheilkunde in Paris erfolgreich zu bestehen.

Schlüsselwörter: Qualitätsmanagement, Strabismus, Schielen, EFQM, Facharztausbildung.

Key words: Quality management, EFQM, residency program, strabismus.

P 64. B. Weingessel, F. Schmid, P. Vécsei-Marlovits (Wien): Prozessmanagement im KAV: Klinische Pfade der Augenabteilung des Krankenhaus Hietzing (Poster) - Process management in KAV: Clinical pathways of the department of ophthalmology, Hietzing Hospital 
Problemstellung: Standardisierung von organisatorischen, diagnostischen und therapeutischen Abläufen auf höchstem wissenschaftlichem Niveau bekommt in der modernen Medizin einen immer höheren Stellenwert. An der Augenabteilung im KH Hietzing werden seit 2008 sämtliche wichtige Abteilungsabläufe mit besonderem Schwerpunkt auf Klinische Pfade im Rahmen des Prozessmanagements erfasst.

Methode und/oder Patienten: Wichtige Krankheitsbilder, Abteilungsabläufe oder häufige diagnostische Prozesse werden von den jeweilig zuständigen SpezialistInnen im Hinblick auf die rezente Literatur und vorhandene Leitlinien durchleuchtet und ein erstes Konzept für den zu erstellenden Prozess erstellt. Im Anschluss wird die Prozessdefinition im Programm Adonis mit Hilfe der MitarbeiterInnen der KAV-IT durchgeführt. Während des wöchentlichen Jour Fixe werden die erarbeiteten Prozesse vorgestellt und vom gesamten Team diskutiert. Nach Abnahme des Prozesses gelten die gemeinsam erarbeiteten Inhalte als verbindlich.

Ergebnisse: Seit 2008 konnten 56 abteilungsspezifische Prozesse erarbeitet werden. Bereits abgenommene Prozesse werden regelmäßig wiederholt und auf Aktualität und Vollständigkeit überprüft. Zu den häufigsten und wichtigsten ophthalmologischen Krankheitsbildern stehen Diagnose- und Therapieprozesse zur Verfügung und erleichtern in einzelnen Spezialgebieten weniger erfahrenen KollegInnen und KollegInnen in Ausbildung die klinische Tätigkeit auf hohem wissenschaftlichem Niveau. Anhand ausgewählter klinischer Pfade wird die Praxisrelevanz aufgezeigt.

Schlussfolgerungen: Die Erstellung Klinischer Pfade ermöglicht eine personenunabhängige, transparente und standardisierte klinische Arbeit unter Berücksichtigung rezenter wissenschaftlicher Erkenntnisse.

Schlüsselwörter: Prozessmanagement, Klinische Pfade.

Key words: Process management, clinical pathway.

P 65. P. Vecsei-Marlovits, P. Irsigler, B. Weingessel (Wien): OP-Qualitätsstatistik an der Augenabteilung im KH Hietzing (Poster) - Quality assurance in ophthalmic surgery at the department of ophthalmology, Hietzing Hospital

Problemstellung: Um qualitativ hochwertige und patientInnenorientierte Arbeit im OP leisten zu können, ist eine kontinuierliche Evaluierung der operativen Leistungen notwendig. Nur so kann bei vermehrtem Auftreten von Komplikationen rechtzeitig eingegriffen und nach den Ursachen gesucht werden. Weiters ist auf diesem Wege die Überprüfung der Lernfortschritte der Beginner in der Ophthalmochirurgie möglich. Nach Neuübernahme der Abteilung wurde die Erhebung der Qualitätsstatistik auch zur Standorterhebung und strukturierten Planung von eventuell notwendigen Änderungen notwendig.

Methode und/oder Patienten: Es wurden sämtliche OP-Berichte von Jänner 2007 bis (zum derzeitigen Stand) Oktober 2010 überprüft. In der Analyse wurde besonderes Augenmerk auf Cataract- und vitreoretinale Operationen gelegt, da einerseits Cataract-Operationen den häufigsten Eingriff an der Augenabteilung im KHR darstellen und andererseits vitreoretinale Operationen, als komplexeste Operation im Bereich der Augenheilkunde, die höchste Komplikations- und ReOperationsrate aufweisen.

Ergebnisse: Von den insgesamt 15.674 Eingriffen wurden 8.757 Cataract-Operationen und 552 vitreoretinale Operationen durchgeführt. Im Bereich der Cataract-Chirurgie konnte die Re-OP-Rate um 73,4\% seit 2007 und um 66,0\% seit 2008 auf 0,48\% gesenkt werden. Bei den vitreoretinalen Operationen wurde 2010 eine Reoperationsrate von 6,8\% erreicht werden, das entspricht einer Senkung der Re-OP-Rate um 86,6\% seit 2007 und um 59,3\% seit 2008 .

Schlussfolgerungen: Bei den Cataract-Operationen führte die Einführung von OP-Tagen, die routinemäßige Assistenz bei den ersten 50-100 Eingriffe von Beginnern durch die Abteilungsvorständin und effizientes intraoperatives Kompli- 
kationsmanagement zu einer deutlichen Senkung der Reoperationsrate. Die Steigerung der OP-Frequenz einzelner OperateurInnen, sowie Lehroperationen und konsequentes intraoperatives Komplikationsmanagement durch die Abteilungsvorständin konnte auch bei vitreoretinalen Operationen die Reoperationsrate deutlich senken.

Schlüsselwörter: Qualität, Statistik, Qualitätsstatistik, Augenoperationen, Komplikationen.

Key words: Quality, quality assurance, eye surgery, complications.

P 66. C. Stappen, K. Rigal, Y. Chen (Wien): Vergleich der Augeninnendruckmessung mittels Pneumo-Tonometrie und Applanationstonometrie bei PatientInnen mit okulärer Hypertension und Glaukom (Poster) - comparison of non-contact versus contact (Goldmann) - measurement of IOP with patients of ocular hypertension and glaucoma

Problemstellung: Die Messung des Augeninnendruckes (IOP) mit einem NonContact-Tonometer als Voruntersuchung vor der augenärztlichen Untersuchung mit anschließender Goldmann Applanationstonometrie (GAT) ist gängige Praxis.

Methode und/oder Patienten: Insgesamt 50 PatientInnen mit okulärer Hypertension und Glaukom wurden in die Studie eingeschlossen. Messungen des IOP erfolgten zuerst mit einer Non-Contact-Tonometrie (Ocular Response AnalyzerORA, Reichert), gefolgt von einer GAT (3 Einzelmessungen) und einer Wiederholung der ORA Messung. Die Meßergebnisse werden verglichen und der drucksenkende Effekt der GAT-Messung selbst wird ermittelt. Zusätzlich ermittelte Variablen sind eine Hornhautpachymetrie und die Hysterese des IOP mittels ORA.

Ergebnisse: Die Meßergebnisse und deren Verhältnis zueinander werden im Hinblick auf die Reihenfolge von Applanations- und Non-Contact-Tonometrie beurteilt und dargestellt.

Schlussfolgerungen: Die Auswertung dieser Daten soll klären ob die beiden Tonometrieverfahren bei PatientInnen mit okulärer Hypertension und Glaukom vergleichbar sind und ob sich die beiden Tonometrieverfahren - ausgeführt in einem kurzen Zeitintervall - gegenseitig beeinflussen können.

Schlüsselwörter: GAT, ORA.

Key words: GAT, ORA.

P 67. D. Schmidl, M. Lasta, S. Kaya, A. Boltz, G. Fuchsjäger-Mayrl, L. Schmetterer (Wien): Effekt der Hemmung von Stickstoffmonoxidsynthase auf die Regulation des Blutflusses der Papille während isometrischer Übung (Poster) - Effect of nitric oxide synthase inhibition on optic nerve head blood flow regulation during isometric exercise in healthy humans

Problemstellung: Autoregulation ist die Fähigkeit eines Gefäßbettes den Blutfluss trotz Änderungen im Perfusionsdruck konstant zu halten. Es konnte gezeigt werden, dass die Retina wie auch die Choroidea über diese Fähigkeit verfügen und dass an dieser Regulation Stickstoffmonoxid (NO) beteiligt ist. In der vorliegenden Studie wurde die Hypothese getestet, ob NO auch auch an der Blutflussregulation in der Papille beteiligt ist. Dazu wurde mittels isometrischer Übung der Blutdruck und damit der okuläre Perfusionsdruck geändert und die Blutflussregulation bestimmt. Um den Effekt von NO zu messen wurde zusätzlich ein NO Synthase Inhibitor verabreicht und mit einen $\alpha$-Rezeptor Agonisten bzw. Placebo verglichen.

Methode und/oder Patienten: 18 gesunde Probanden wurden in diese randomisierte, doppelblinde, placebo-kontrollierte, dreifach Crossover Studie aufgenommen. An drei Studientagen erhielten diese entweder NG-monomethyl-L-arginine (L-NMMA, NOS Inhibitor), Phenylephrin ( $\alpha$-Rezeptor Agonist) oder Placebo. Während diese Medikamente intravenös verabreicht wurden, nahmen die Probanden für 6 Minuten die Hockestellung ein. Der Blutfluss in der Papille 
wurde dabei mittels Laser Doppler Flowmetry gemessen. Der okuläre Perfusionsdruck wurde als Differenz zwischen mittleren arteriellen Druck und Intraokulardruck kalkuliert.

Ergebnisse: Nach Verabreichung von L-NMMA und Phenylephrin wurde eine signifikante Erhöhung des basalen okulären Perfusionsdruckes festgestellt ( $\mathrm{p}<$ 0,001). Eine Reduktion des basalen Blutflusses in der Papille wurde jedoch nur nach Verabreichung von L-NMMA beobachtet $(p=0,02)$. Der Anstieg des okulären Perfusionsdrucks während isometrischer Übung war unabhängig von der verabreichten Medikation ( $\mathrm{p}=0,69$ ). Auch die Änderungen des Blutflusses in der Papille waren vergleichbar mit allen drei verabreichten Medikamenten $(p=0,43)$.

Schlussfolgerungen: Diese Daten weisen darauf hin, dass NO eine bedeutende Funktion bei der Regulation des basalen Blutflusses in der Papille hat, jedoch nicht an der Blutflussregulierung während isometrischer Übung beteiligt zu sein scheint.

Schlüsselwörter: Stickstoffmonoxid, Autoregulation, Laser Doppler Flowmetry, Blutfluss, Papille.

Key words: Nitric oxide, autoregulation, laser Doppler flowmetry, blood flow, optic nerve head.

P 68. B. Teuchner, E. Schmid, J. Troger, N. Bechrakis (Innsbruck): Reversible Papillenexcavation nach erfolgreicher Trabekulektomie (Poster) - Reversal of optic disc cupping after trabeculectomy

Problemstellung: Die Reversibilität einer glaukomatösen Papillenexcavation nach einer Augendruckreduktion ist ein allgemein bekanntes Phänomen, das bei juvenilem Glaukom ausgeprägter zu finden ist, als beim chronischen Offenwinkelglaukom. Durch verbesserte Imagingmethoden sind diese Veränderungen besser dokumentierbar und quantifizierbar geworden. Wir beschreiben einen Patienten bei dem nach erfolgreicher Trabekulektomie, eine deutliche, mittels Papillenfotographie dokumentierte Veränderung der Papillenmorphologie beobachtete werden konnte.

Methode und/oder Patienten: Die routinemässig bei all unseren Glaukompatienten durchgeführten Imagingmethoden wie Papillenfotographie, HRT und GDX-VCC wurden bei einem 52-jährigen Patienten prä - sowie 6 und 12 Monate postoperativ nach einer Trabekulektomie miteinander verglichen.

Ergebnisse: Bereits 6 Monate nach erfolgreicher Trabekulektomie mit einer Drucksenkung von $60 \%$ zeigte sich beim Vergleich der prä- und postoperativen Papillenfotos eine deutliche Rückbildung der Papillenexcavation, eine Zunahme des Randsaumvolumens und ein deutlich veränderter Verlauf der Gefäße im Bereich des neuroretinalen Randsaums. Mit dem HRT konnten diesen Veränderungen quantifiziert werden.

Schlussfolgerungen: Die Reversibilität einer glaukomatösen Papillenexcavation nach Augendrucksenkung konnte in diesem Fall demonstriert werden; in wieweit diese morphologischen Veränderungen an der Papille auch funktionelle Veränderungen des Gesichtsfeldes nachsichziehen, wird in der Literatur noch sehr kontroversiell diskutiert.

Schlüsselwörter: Papillenexcavation, Reversibilität, Trabekulektomie, Papillenfotographie.

Key words: Optic disc cupping, reversal of cupping, trabeculectomy, optic disc photography.

P 69. K. Rigal, C. Stappen, Y. Chen, S. Tatzreiter, O. Findl (Wien): Dynamische intraoperative Schnittbilddarstellung des vorderen Augenabschnitts mittels OCT bei Trabekulektomie (Poster) - Dynamic intraoperative imaging of the anterior eye-segment by OCT during trabeculectomy

Problemstellung: Die Verbindung des hoch auflösenden Visante OCT mit dem Operationsmikroskop OPMI Visu 200 über eine Fiberoptik (Carl Zeiss Meditec AG, Jena) lässt die Darstellung des vorderen Augenabschnittes intraoperativ
Bemerkungen 
während chirurgischer Eingriffe im vorderen Augenabschnitt zu.

Methode und/oder Patienten: Fallbeispiele von Trabekulektomien mit der Darstellung intraoperativer Veränderungen des vorderen Augenabschnittes werden aufgezeichnet.

Ergebnisse: Videounterstützte Darstellung der intraoperativen Abläufe im vorderen Augenabschnitt während Trabekulektomie wird präesentiert.

Schlussfolgerungen: Über eine Verbindung zwischen OCT und Operationsmikroskop durch eine Fiberoptik lassen sich intraoperative Abläufe im vorderen Augenabschnitt bei Trabekulektomie darstellen.

Schlüsselwörter: OCT, intraoperative Schnittbilddarstellung, Trabekulektomie. Key words: OCT, intraoperative imaging, trabeculectomy.

P 70. C. Runge, C. Strohmaier, B. Bogner, A. Trost, F. Schrödl, G. Grabner, H. Reitsamer (Salzburg): Auswirkung von Adenosin auf die Durchblutung am Sehnervenkopf in anästhesierten Kaninchen (Poster) - The effect of Adenosine on the optic nerve head blood flow in rabbits

Problemstellung: Untersuchung der Wirkung von Adenosin, in verschiedenen Konzentration, auf die Durchblutung im Sehnervenkopf in anästhesierten weißen Neuseeländer Kaninchen mittels nicht-invasiver Laser Doppler Flowmetry.

Methode und/oder Patienten: Weiße Neuseeländer Kaninchen $(n=6)$ wurden mit Pentobarbital (50 mg/kg, iv.) anästhesiert und mit Raumluft beatmet. Der mittlere arterielle Druck (MAP) und der mittlere intraokuläre Druck (IOP) wurden mittels Kanülierung der zentralen Ohrarterie beziehungsweise des Glaskörpers gemessen. Eine kontinuierliche Messung der Durchblutung im Sehnervenkopf wurde mittels nicht invasiver Laser Doppler Flowmetrie durchgeführt. Adenosine wurde als i.v. Infusion in den Konzentrationen 2,5 mg/kg/h, $5 \mathrm{mg} / \mathrm{kg} / \mathrm{h}$, $7,5 \mathrm{mg} / \mathrm{kg} / \mathrm{h}$ und $10 \mathrm{mg} / \mathrm{kg} / \mathrm{h}$ verabreicht.

Ergebnisse: Adenosin bewirkte eine signifikante Vasodilatation (Abnahme des Gefäßwiderstands um 32\%) bei einer Dosis von 7,5 mg/kg/h, die durch die Dosiserhöhung auf $10 \mathrm{mg} / \mathrm{kg} / \mathrm{h}$ nicht mehr signifikant gesteigert werden konnte. Bei dieser Dosis wurde der Augendruck signifikant um 34\% (13,36 \pm 0,51 mmHg auf $17,89 \pm 1,13 \mathrm{mmHg}$ ) erhöht. Bei der höchsten Adenosin Dosis von $10 \mathrm{mg} / \mathrm{kg} / \mathrm{h}$ wurde der Blutdruck signifikant gesenkt.

Schlussfolgerungen: Es konnte eine signifikante konzentrationsabhängige Vasodilatation im Bereich des Sehnervenkopfes nach Verabreichung von Adenosin festgestellt werden. Diese Ergebnisse zur Wirkung von Adenosin sind sehr gut vergleichbar mit in der Literatur und von unserer Arbeitsgruppe durchgeführten Untersuchungen mit anderen Messtechniken zur Bestimmung der Durchblutung.

Schlüsselwörter: Adenosin, Sehnervenkopf, Durchblutung.

Key words: Adenosine, optic nerv head, blood supply.

P 71. B. Bogner, C. Runge, F. Schrödl, C. Strohmaier, A. Trost, G. Grabner, H. Reitsamer (Salzburg): Der Einfluss von V1- und V2-Rezeptor Antagonisten auf den okulären Blutfluss (Poster) - The Effect of V1- and V2-Receptor Antagonists on ocular blood flow

Problemstellung: In-vivo studies have shown that Vasopressin (AVP) is a highly potent vasoconstrictor in the eye. Immunohistochemistry proved the presence of V1- and V2-receptors in choroidal and ciliary blood vessels. The aim of this study is the functional characterization of the vasopressin-receptor subtypes involved in blood flow regulation in the choroidal and ciliary vascular bed in the acute rabbit model.

Methode und/oder Patienten: In anesthetized rabbits $(n=6)$ mean arterial pressure (MAP), intraocular pressure (IOP) and orbital venous pressure (OVP) were measured by direct cannulation of the central ear artery, the vitreous, and the orbital venous sinus, respectively. To change the perfusion pressure (PP) over a wide range, MAP was manipulated mechanically via occluders placed around the aorta and the inferior vena cava. Laser Doppler flowmetry was used to record 
choroidal blood flow (ChorBF) and ciliary blood flow (CilBF) continuously. To induce vasoconstriction after baseline measurements arginine-vasopressin (AVP) (1,3 ng/kg/min) was applied intravenously. A V1-receptor antagonist ([beta-Mercapto-beta,beta-cyclopentamethylenepropionyl1, O-me-Tyr2, Arg8]-Vasopressin) and a V2-receptor antagonist ([Adamantaneacetyll, O-Et-D-Tyr2, Val4, Aminobutyryl6, Arg8,9]-Vasopressin) were given by intravenous bolus injection. Ergebnisse: The vasoconstrictive response to AVP caused a $20 \%$ reduction of ChorBF, which could be blocked by the V1-receptor antagonist $(p<0.001)$, whereas the administration of the V2-receptor antagonist had no effect (n. s.). In the ciliary body the AVP-induced vasocostriction was slightly higher than in the choroid. Nevertheless, the response to the applied antagonists resembles that of the choroid.

Schlussfolgerungen: Although V1 and V2-receptors are both expressed in ocular vessels, the functional data suggest that the AVP-induced vasoconstriction in ocular vessels is caused by V1-receptor mediated signaling pathways. The V2antagonist showed no effect at the selected concentrations. Further studies have to prove if different concentrations of AVP and V2-antagonist influence ocular blood flow, respectively.

Schlüsselwörter: Vasopressin Rezeptor Antagonisten, choroidaler Blutfluss, Ziliarkörperdurchblutung.

Key words: Vasopressin receptor antagonists, choroidal blood flow, ciliary blood flow.

P 72. A. Trost, F. Schrödl, B. Bogner, C. Strohmaier, G. Grabner, L. Aigner, H. Reitsamer (Salzburg): Differenzierungspotential retinaler und choroidaler Perizyten (Poster) - Differentiation potential of retinal and choroidal pericytes

Problemstellung: Pericytes, cells closely adjacent to blood vessels, are important for vascular development, stabilization and remodelling. They exhibit hallmarks of mesenchymal stem cell identity and develop to osteogenic, chondrogenic, adipogenic and myogenic lineages in vitro. Here, we aimed to verify this multipotent capacity for retinal and choroidal pericytes and moreover tested their neuronal transdifferentiation potential.

Methode und/oder Patienten: Pericytes were isolated from bovine retina and choroid, using an optimized enzymatic digestion protocol. Primary retinal and choroidal cells were characterized with a panel of pericyte specific markers using immunhistochemistry. Differentiation into adipocytes, chondrocytes and osteoblasts was performed for 4 weeks in specific corresponding differentiation media. The differentiation assays were stained with RedOilO, alkaline phosphatase (alizarin) and alcian blue $\mathrm{pH} 2$. Additionally mRNA expression analyses were conducted to verify the respective cell types.

Ergebnisse: Within 2 weeks, retinal pericytes differentiated into chondrocytes and osteoblasts in the corresponding media, while adipocyte differentiation was observed spontaneously during the first two passages in normal cultivation media. The differentiation ability of choroidal pericytes was similar to retinal ones but at slower growth rates, but they showed no differentiation into adipocytes. Furthermore choroidal pericytes developed a neuronal-like phenotype. Osteogenic and chondrogenic activity was proven by marker genes on mRNA level via real-time PCR.

Schlussfolgerungen: Retinal as well as choroidal pericytes posses a mesenchymal stem cell like capacity and differentiated into chondrocytes and osteoblasts. Of special interest is the neuronal-like phenotype of choroidal pericytes, being a possible source of cells for a glaucoma therapy model.

Schlüsselwörter: Perizyten Differenzierung, Chondrozyten, Osteoblasten.

Key words: Pericyte differentiation, chondrocytes, osteoblasts. 
P 73. E. Schmid, J. Troger, M. Nogalo, B. Teuchner, R. Fischer-Colbrie, G. Sperk, N. Bechrakis (Innsbruck): Substanz P, Sekretoneurin, Neuropeptid Y und VEGF in einem Hyperoxie/Hypoxie Modell in der Retina der Maus (Poster) - Substance P, secretoneurin, neuropeptide Y and VEGF in a hyperoxia/ hypoxia model in the mouse retina

Problemstellung: Für das Auftreten von Neovaskularisationsprozessen wird vor allem vascular endothelial growth factor (VEGF) verantwortlich gemacht. Das Ziel dieser Studie war herauszufinden, ob die Neuropeptide Substanz P, Sekretoneurin und Neuropeptid Y in der Pathogenese von retinalen Neovaskularisationen im Hyperoxie/Hypoxie-Modell der Maus involviert sind. Diese drei Neuropeptide weisen ausgeprägte proangiogene Eigenschaften auf und ein Anstieg der Expression durch die relative Hypoxie wäre zwar kein Beweis aber doch ein eindeutiger Hinweis für deren Beteiligung.

Methode und/oder Patienten: Es wurden Würfe von C57 BL/6 Muttermäusen verwendet. Diese Jungmäuse wurden vom 7. postnatalen Tag an für 5 Tage einer Konzentration von $75 \%$ Sauerstoff ausgesetzt und danach wieder in Normalluft gebracht, wodurch eine relative Hypoxie entsteht. Die Tiere wurden an P9, P11, P12, P13, P14, P16 und P20 durch eine Überdosis von $\mathrm{CO}_{2}$ getötet, die Bulbi enukleiert und die Retina freipräpariert. Altersneutrale unbehandelte Tiere dienten als Kontrolle. Die Konzentration der Peptide wurde in der Retina mittels Radioimmunoassay und die Konzentration von VEGF mittels ELISA bestimmt.

Ergebnisse: Es konnte festgestellt werden, dass die Spiegel von VEGF durch Hyperoxie massiv abfielen und dass die Konzentration durch relative Hypoxie signifikant anstieg. Die Konzentration von Substanz P und noch stärker ausgeprägt von Neuropeptid Y fiel durch Hyperoxie leicht ab und durch relative Hypoxie kam es zu einer verzögerten Normalisierung der Spiegel. Für Sekretoneurin war kein Effekt feststellbar.

Schlussfolgerungen: Die Down-Regulation der Expression von VEGF durch Hyperoxie und der massive Anstieg durch relative Hypoxie weist darauf hin, dass VEGF ein wichtiger Mediator in der Pathogenese von retinalen Neovaskularisationen bei diesem Hyperoxie/Hypoxie-Modell ist. Der leicht reduzierende Effekt von Hyperoxie auf SP und NPY und die verzögerte Normalisierung der Spiegel unter relativer Hypoxie kommt möglicherweise durch eine vorübergehende Reduktion der Expression im Sinne einer Down-Regulation zustande. Aus diesen Ergebnissen kann man schließen, dass Substanz P, Neuropeptid Y und Sekretoneurin nicht in der Pathogenese von Neovaskularisationen in diesem Tiermodell beteiligt sind.

Schlüsselwörter: Substanz P, Sekretoneurin, Neuropeptid Y, Hyperoxie/Hypoxie Modell, Mausretina, Neovaskularisation, Angiogenese.

Key words: Substance P, secretoneurin, neuropeptide Y, hyperoxia/hypoxia model, mouse retina, neovascularisation, angiogenesis.

P 74. F. Schrödl, A. Trost, C. Strohmaier, B. Bogner, G. Grabner, B. Kofler, H. Reitsamer (Salzburg): Vorhandensein des Neuropeptids Alarin im Auge verschiedener Spezies (Poster) - Existence of the neuropeptide alarin in the eye of various species

Problemstellung: Alarin is a lately discovered neuropeptide of the galanin peptide family with vasoconstrictor acitivity in murine skin. In the eye, ocular blood flow and aqueous humor production are essential for ocular homeostasis and a pool of intrinsic and extrinsic neuropeptides is involved to fulfill this task. Alarin might be an additional regulatory peptide in these processes.

Methode und/oder Patienten: Eyes of human (meeting the Declaration of Helsinki), mouse, and rat were processed for immunohistochemistry against alarin using affinity purified antibodies. Confocal laser-scanning microscopy was used for documentation and quantitative RT-PCR was performed to detect alarin mRNA expression in human eyes.

Ergebnisse: Alarin-like immunoreactivity (alarin-LI) was detected in corneal epi- 
and endothelium of human, mouse, and rat, as well as in the conjunctiva of mouse and rat. Alarin-LI was found in the iris of all the species investigated and, in humans, was concentrated around blood vessels. All three species showed distinctive alarin-LI in the non-pigmented epithelium of the ciliary body. In the retina of mouse and rat, maximum signals were detected in the outer nulear and opticus ganglion cell layer, whereas in humans a strong alarin-LI was found around retinal blood vessels and in intrinsic choroidal neurons (ICN). Quantitative RT-PCR in human revealed higher alarin mRNA expression in the choroid than retina.

Schlussfolgerungen: Alarin is widely distributed in the eyes of species investigated. The existence around blood vessels/ in ICN might indicate an involvement in ocular blood flow regulation. Alarin in the non-pigmented epithelium of the ciliary body might be involved in aqueous humor production.

Schlüsselwörter: Neuropeptid, Immunohistochemie, konfokale Mikroskopie, Blutflussregulation, Kammerwasser Produktion.

Key words: Neuropetide, immunohistochemistry, confocal microscopy, blood flow, aqueous humor production.

P 75. S. Scholtz, G. Auffarth (Heidelberg): 1911 - Ein Ophthalmologe erhält den Nobelpreis: Allvar Gullstrand, Wissenschaftler, Mathematiker und kreativer Erfinder (Poster) - 1911 - An ophthalmologist won the Nobel price: Allvar Gullstrand, surgeon, mathematician and creative inventor

Problemstellung: Allvar Gullstrand (1862-1930) leistete wertvolle Pionierarbeit auf den Gebieten der theoretischen Optik und leitete wegweisende Forschungsprojekte über die physikalischen und mathematischen Eigenschaften des menschlichen Auges. „Nebenbei“ erfand er wesentlich ophthalmologische Untersuchungsgeräte wie die erste Spaltlampe, deren Prinzipien noch heute im Einsatz sind.

Methode und/oder Patienten: Literaturrecherche in Fachpublikationen via PubMed, Google Scholar und Google.

Ergebnisse: Gullstrand nutzte methodische Kenntnisse der physikalischen Mathematik zur wissenschaftlichen Untersuchung von Bildentstehung und Lichtbrechung des Auges. In Anerkennung seiner Forschungen wurde ihm 1911 der Nobel Preis in Physiologie oder Medizin ebenso wie zahlreiche weitere Ehrungen zuteil. Gullstrand erwies sich auch auf den Gebieten der Astigmatismusforschung, Verbesserung des Ophthalmoskops oder der korrektiven Versorgung von Katarakt-Operierten als herausragender Forscher. Sein schematisches Modell des Auges, das Gullstrand Auge, wird bis heute als Referenzobjekt verwendet.

Schlussfolgerungen: Gullstrand's Forschung brachte der Ophthalmologie den detaillierten Aufbau und dessen Funktionsweise näher. Bis heute gelten seine Erfindungen im Bereich der Ophthalmologie als Gold-Standard, an denen sich heutige Entwicklungen messen lassen müssen.

Schlüsselwörter: Allvar Gullstrand, Medizingeschichte, Nobel-Preis, 1911. Key words: Allvar Gullstrand, history of medicine, Nobel Price, 1911. 


\section{STATE OF THE ART: Tumore}

Moderation: F. J. Steinkogler, G. Langmann

S 1 Franz J. Steinkogler (Wien): Diagnose und Therapie von Lidtumoren

S 2 Jack Rootman (Toronto): Management of Orbital tumors

S 3 Gerald Langmann (Graz): Diagnose und Therapie des Retinoblastoms

S 4 Georg Blatsios (Innsbruck): Diagnostik intraokularer Tumore

S 5 Gerald Langmann (Graz): Therapieoptionen beim Aderhautmelanom

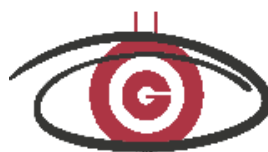

OSTERREICHISCHE OPHTHALMOIOGTSCHF GES LI.ISCIIAF'
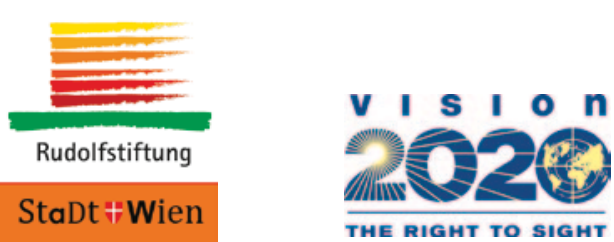

\section{ÄRZTEFORTBILDUNG}

\section{KONTROVERSIELLES IN DER AUGENHEILKUNDE}

\section{Mittwoch • 28. September $2011 \cdot 8.30-13.00$ Uhr $\bullet$ Wiener Rathaus $\bullet$ Nordbuffet}

Die Fortbildung ist für das DFP der ÖÄK approbiert. Keine Teilnahmegebühr.

Anmeldung: MEDEVENT T: +43154538 10•E-Mail: medevent@medevent.cc·www.medevent.cc

Veranstalter: Österreichische Ophthalmologische Gesellschaft Wissenschaftliche Leitung: Univ.-Prof. ${ }^{\text {in }}$ Dr. ${ }^{\text {in }}$ Susanne Binder

\section{Parallelveranstaltung: 2. Wiener Augentag}

Populärmedizinische Veranstaltung für Betroffene und Interessierte Mittwoch • 28. September 2011 • $10.00-18.00$ Uhr $•$ Wiener Rathaus $•$ Festsaal 


\section{VERZEICHNIS DER VORTRAGENDEN}

ALLEGRI, P. (Lavagna/Genua) . . . . . . . . . . . 102

BACHERNEGG, A. (Salzburg) . . . . . . . . . . . . . . 124

BECHRAKIS, N. (Innsbruck) . . . . . . . . . . . 155

BERNHART, A. (Wien) . . . . . . . . . . . . . . . 157

BOGNER, B. (Salzburg) . . . . . . . . . . . . . 89, 190

BOLTZ, A. (Wien). . . . . . . . . . . . . . . . . . . 108

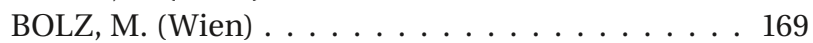

BORKENSTEIN, A. (Graz) . . . . . . . . . . . . . . 147

BRANDTNER, H. (Salzburg). . . . . . . . . . . . . . 93

BÜHL, W. (Wien) . . . . . . . . . . . . . . . . . . 166

CHEN, Y. (Wien) . . . . . . . . . . . . . . . . . . 182

DEKANOVIC, S. (Graz) . . . . . . . . . . . . . . . 159

DEXL, A. (Salzburg) . . . . . . . . . . . . . . . . . . . . . 118

DIMMER, A. (Innsbruck) . . . . . . . . . . . . 150

DIRISAMER, M. (Linz) . . . . . . . . . . . 110, 113

EGGER, S. (Salzburg) . . . . . . . . . . . . . . . . . . . 138

EMESZ, M. (Salzburg). . . . . . . . . . . . . . . . . 98

EISENKÖLBL, S. (Wien). . . . . . . . . . . . . . . . . . . . . . . . . .

ETTL, A. (St. Pölten) . . . . . . . . . . . . . . . . . . 99

FALKNER-RADLER, C. (Wien) . . . . . . . . . . 137

FEJES, I. (Szeged) . . . . . . . . . . . . . . . . . . . . . . . . . . . . . . . . . .

FINDL, O. (Wien). . . . . . . . . . . . . . . . . . . . . . . 177

FISCHER, C. (Salzburg). . . . . . . . . . . . . . 100

FUCHSJÄGER-MAYRL, G. (Wien) . . . . . . . . . 170

GARAS, A. (Budapest) . . . . . . . . . . . . . . . . . . 995

GEORGI, T. (Graz) . . . . . . . . . . . . . . . . . 131

GEORGOPOULOS, M. (Wien) . . . . . . . . . . . 132, 177

GLITTENBERG, C. (Wien) . . . . . . . . . . . . . 169

HAAS, G. (Innsbruck) . . . . . . . . . . . . . . . . . . . . . 163

HAAS, P. (Wien) . . . . . . . . . . . . . . . . . 129

HAGEN, S. (Wien) . . . . . . . . . . . . . . . . 129

HARTLIEB, E. (Innsbruck) . . . . . . . . . . . . . 148

HAUSBERGER, S. (Graz) . . . . . . . . . . . . . . . . . . . . . . . 169

HESSE, R. (Graz) . . . . . . . . . . . . . . . . . . 154

HIRNSCHALL, N. (Wien). . . . . . . . . . . . . . . . . . 127

HITZL, W. (Salzburg) . . . . . . . . . . . . . . . . . . 107

HOCHWARTER, A. (Wien) . . . . . . . . . . . . . 142

HOHENSINN, M. (Salzburg). . . . . . . . . . . . . . 123

HUBER, S. (Innsbruck) . . . . . . . . . . . . . . 183

IVASTINOVIC, D. (Graz) . . . . . . . . . . . . . 126

JAMPOL, L. (Chicago) . . . . . . . . . . . . . . 102

KAHRAMAN, G. (Wien) . . . . . . . . . . . . . 126

KAYA, S. (Wien). . . . . . . . . . . . . . . . . 167

KERNSTOCK, C. (Tübingen). . . . . . . . . . . . . 137

KIESELBACH, G. (Innsbruck) . . . . . . . . . . . . . . 104

KISS, C. (Wien) . . . . . . . . . . . . . . . . . . 130

KOVÁCS, I. (Budapest San Juan de Alicante) . . . . . . 96
KRÁNITZ, K. (Budapest) . . . . . . . . . . . . . . . . . 97

KRIECHBAUM, K. (Wien) . . . . . . . . . . . . . 161

KROISAMER, J. (Wien) . . . . . . . . . . . . . . . 132

LAMMER, J. (Wien) . . . . . . . . . . . . . . . . . . 160

LASTA, M. (Wien) . . . . . . . . . . . . . . . . . . . 165

LINDNER, E. (Klagenfurt) . . . . . . . . . . . . . . 101

LORENZ, K. (Mainz) . . . . . . . . . . . . . . . . . . . . . . . . . . . .

LÜBKE, B. (Linz) . . . . . . . . . . . . . . . . . . . 122

MARVAN, P. (Salzburg) . . . . . . . . . . . . . . . . . . . . . . 98

MAYER, C. (Graz) . . . . . . . . . . . . . . . . . . . . . 186

MAYR-SPONER, U. (Wien) . . . . . . . . . . . . . . 158

MENNEL, S. (Marburg). . . . . . . . . . . . . . . . 167

MITSCH, C. (Wien) . . . . . . . . . . . . . . . . 133

MUNK, M. (Wien) . . . . . . . . . . . . . . . . . 144

MYLONAS, G. (Wien) . . . . . . . . . . . . . . . 173

NEPP, J. (Wien) . . . . . . . . . . . . . . . . . . . . . . . . . . 153

NISCHLER, C. (Salzburg) . . . . . . . . . . . . 105

PAHOR, D. (Maribor) . . . . . . . . . . . . . . . . . . . 175

PALKOVITS, S. (Wien). . . . . . . . . . . . . . . . . . . . . . . . . . . . . . . . . . . . . . . . . .

PEMP, B. (Wien) . . . . . . . . . . . . . . . . . . 164

PETROVSKI, G. (Debrecen Oslo) . . . . . . . . . . . . 94

PICHLER-RUBENZUCKER, E. (Steyr) . . . . . . . . 150 PIEH, S. (Wien) . . . . . . . . . . . . . . . 120, 178, 179 POLLREISZ, A. (Wien) . . . . . . . . . . . . . . 168 POPP, N. (Wien) . . . . . . . . . . . . . . . 153 PRAGER, F. (Wien) . . . . . . . . . . . . . . . . . . . . . . 113 PRAGER, S. (Wien) . . . . . . . . . . . . . . . 136 PRINZ, A. (Wien) . . . . . . . . . . . . . . . . . . . . . . . . . . . . . . . . . . . . . PUTZ, D. (Innsbruck) . . . . . . . . . . . . . . 147

RASP, M. (Salzburg) . . . . . . . . . . . . . . . . . . . . . . . . . 176 RAU, M. (Cham) . . . . . . . . . . . . . . . . 178 REDLBERGER, A. (Salzburg) . . . . . . . . . . . 100 REISCHL, V. (Salzburg) . . . . . . . . . . . . . . 123, 125 REITER, C. (Würzburg) . . . . . . . . . . . . . . . . 171 REITSAMER, H. (Salzburg) … . . . . . . 90, 105, 107

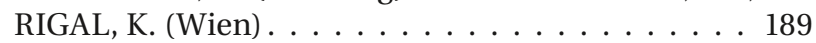
RIHA, W. (Salzburg) . . . . . . . . . . . . . . . . . . 117 ROBERTS, P. (Wien) . . . . . . . . . . . . . . . . . . . . . 143 RUCKHOFER, J. (Salzburg) . . . . . . . . . . . . 121 RÜCKL, T. (Salzburg) . . . . . . . . . . . . . . . . . 116, 119 RUNGE, C. (Salzburg) . . . . . . . . . . . . . 92, 190

SACU, S. (Wien). . . . . . . . . . . . . . . . . . 141

SCHERER, K. (Karlsruhe) . . . . . . . . . . . . . . . . . . . . . . . . . . . . SCHESCHY-LUN, U. (Wien) . . . . . . . . . . . . . . . . . 157 SCHLANITZ, F. (Wien) . . . . . . . . . . . . . . . . . . . 135

SCHMID, E. (Innsbruck) . . . . . . . . . . . . . . . . . . 192 SCHMIDINGER, G. (Wien) . . . . . . . . . . . . . 111 SCHMIDL, D. (Wien) . . . . . . . . . . . . . . . . . . . . 188 SCHMIDT, J. (Duisburg) . . . . . . . . . . . . . . . . . . 139 SCHMIDT-ERFURTH, U. (Wien) . . . . . . . . . . . . . 128 SCHOLDA, C. (Wien) . . . . . . . . . . . . . . 139 
SCHÖLLBAUER, V. (Wien) . . . . . . . . . . . . . . . 184 SCHOLLER, A. (Wien) . . . . . . . . . . . . . . . 173 SCHOLTZ, S. (Ettlingen) . . . . . . . . . . . . . . . . . 193 SCHÖNHERR, U. (Linz) . . . . . . . . . . . . . 140 SCHRIEFL, S. (Wien) . . . . . . . . . . . . . . . . . 174 SCHRÖDL, F. (Salzburg) . . . . . . . . . . . . . . . 91, 192

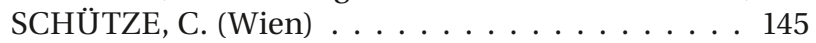
SCHWAB, C. (Graz) . . . . . . . . . . . . . . . . . 142 SEIDEL, G. (Graz) . . . . . . . . . . . . . . . 130, 149 SEYEDDAIN, O. (Salzburg) . . . . . . . . . . 111 SIMADER, C. (Wien) . . . . . . . . . . . . . . . . . 134 SMRETSCHNIG, E. (Wien) . . . . . . . . . . . . . 180 STANGLER-ZUSCHROTT, E. (Wien) . . . . . . . . . 103 STAPPEN, C. (Wien) . . . . . . . . . . . . . . . . . . . 188 STATTIN, M. (Innsbruck) . . . . . . . . . . . . 159 STAURENGHI, G. (Milano) . . . . . . . . . . . . . 162 STEGER, B. (Innsbruck) . . . . . . . . . . . . . . . . 109 STOIBER, J. (Salzburg) . . . . . . . . . . . . . 112, 114 STRASSER-SCHRATTENTHALER, M. (Innsbruck). . 152,

STRAUSS, R. (Linz) ............... 110 STROHMAIER, C. (Salzburg) . . . . . . . . . . 91, 93 SULZBACHER, F. (Wien) .
TATZREITER, S. (Wien). . . . . . . . . . . . . . . . . . 181 TEUCHNER, B. (Innsbruck) . . . . . . . . . . . . . . 189 THEURL, M. (Innsbruck) . . . . . . . . . . . . 156 THIMM, C. (Duisburg) . . . . . . . . . . . . . . 165 TOTH-KOVACS, K. (Pecs) . . . . . . . . . . . . 96 TROST, A. (Salzburg) . . . . . . . . . . . . . . 89, 191

VECSEI-MARLOVITS, P. (Wien) . . . . . . . . . . 187

WAGNER, J. (Wien) . . . . . . . . . . . . . . . . 151

WANDERER, S. (Wien) . . . . . . . . . . . . . . . 179

WEBER, N. (Marburg). . . . . . . . . . . . . . . . 180

WEDRICH, A. (Graz). . . . . . . . . . . . . . . . . 184

WEIGERT, G. (Wien) . . . . . . . . . . . . . . . . . . 155

WEINGESSEL, B. (Wien) . . . . . . . . . . . . . 186

WEISS, K. (Klagenfurt) . . . . . . . . . . . . . . . 152

WERNER-STÖLLINGER, M. (Salzburg) . . . . . . . . 103

WINTERSTELLER, C. (Salzburg) . . . . . . . . . . 106

WOHLFART, C. (Graz) . . . . . . . . . . . . . . . . . 185

WOLLINGER, C. (Wien). . . . . . . . . . . . . . . 182

ZEHETNER, C. (Innsbruck) . . . . . . . . . . . 163 


\section{INDUSTRIEAUSSTELLUNG}

AGEPHA GmbH

ALCON Ophthalmica GmbH

ALFAMED medizinprodukte gmbh

AMO Germany GmbH, Betriebsstätte

Österreich

ANDRÉ - E \& W Scheffknecht Betriebs GmbH

Argus individuell Optic GmbH

ASKIN und Co GmbH

Augenklinik Dardenne

Bausch \& Lomb

Bilosa Handels GmbH

Brumaba GmbH\&Co JG

Carl ZEISS GmbH

CIBA VISION GmbH

CJOB

Contactlinseninstitut Miller GmbH, HECHT

Contactlinsen Österreich

CR-MEDICAL Ophthalmology GmbH

CROMA PHARMA GmbH

Domilens GmbH

Dr. Gerhard Mann chem.-pharm.Fabrik GmbH

Dr. Schmidt Intraocularlinsen $\mathrm{GmbH}$

ECA Medical HandelsGmbH

GALIFA CONTACTLINSEN Gmbh

Geodis Austria GmbH

Hetych Kontaktlinsen KG

Hoya Surgical Optics GmbH

IFA-Systems AG

Johnson \& Johnson Vision Care

Lenuspharma

LICHT für die Welt (Christoffel)

LOBMAIER Datentechnik

med lab

medilens ag

MENICON GmbH

Merck Sharp \& Dohme (MSD)

NEUMED AG

NOVARTIS Pharma GmbH

OctreoPharm GmbH

Omnivision $\mathrm{GmbH}$

Optima Pharmazeutische $\mathrm{GmbH}$

Optos GmbH

Ordinationstechnik und Beschaffungs-

management

Pfizer Corporation Austria Ges.m.b.H.

PHARM ALLERGAN GmbH

PHARMASELECT Handels GmbH

PLUSOPTIX GmbH

POLYTECH Ophthalmologie $\mathrm{GmbH}$

Rayner Surgical GmbH

SCHWEIZER OPTIK Ges.m.b.H.

TASCHNER CONTACTLINSEN Ges.m.b.H.

TRB Chemedica (Austria) GmbH

WIRMSBERGER \& BÖHM Fachbuchhandlung

für Medizin $\mathrm{GmbH}$
Simmeringer Hauptstraße 24

Mariahilferstraße 120

Daniel Granstraße 57

Nottendorfergasse 11

Schwefel 93

Hermann Oberth Straße 18A

Albert Schweitzergasse 6

Friedrich-Ebert-Straße 23-25

Brunsbütteler Damm 165-173

Frachtenbahnhof, 1. Auffahrt

Bgm.-Finsterwalder-Ring 21

Modecenterstraße 16

Stella-Klein-Löw-Weg 17

Enenkelstraße 11 und 13/9

Meranerstraße 3

Aigen 21

Stockerauerstraße 181

Holsteiner Chaussee 303a

Postfach 2516

Westerwaldstraße 11-13

Niederhart 100

Postfach 100

Liebemannstraße A01 304/7

Ortsstraße 277b

Lyoner Straße 24-26

Augustinusstraße 11 B

Vorgartenstrasse 206B

Seeböckgasse 59

Niederhofstraße 26-28

Schildorn 92

Wolfgang-Pauli-Gasse 5

Bahnhofstraße 5

Odenwaldring 44

Donau City Straße 6

Bahnstraße 16

Stella-Klein-Löw-Weg 17

Am Schlossberg

Eichenstraße 32

Wittibsmühle 5

Werner-von-Siemens-Straße 2-6

Zieglergasse 81

Florido Tower, Floridsdorfer Hauptstr. 1 A-1210 Wien

Twin Tower 12 A, Wienerbergstraße 11 A-1100 Wien

Ernst-Melchior-Straße 20

Neumeyerstraße 48

Arheilger Weg 6

Kirschäckerstraße 25

Am Steinberg 200

Linzerstraße 16

IZ NÖ-Süd Straße 7/58 D/1/2.OG

Hufelandstraße 13
A-1110 Wien
A-1060 Wien
A-3100 St. Pölten
A-1030 Wien

A-6850 Dornbirn

D-85640 Putzbrunn

A-1140 Wien

D-53177 Bonn

D-13581 Berlin

A-6020 Innsbruck

D-82515 Wolfratshausen

A-1034 Wien

A-1020 Wien

A-1160 Wien

A-6020 Innsbruck

A-4204 Haibach i. Mkr

A-2100 Korneuburg

D-22457 Hamburg

D-36243 Niederaula

D-53757 Sankt Augustin

A-6265 Hart

A-6973 Höchst

A-2345 Brunn/Geb.

A-2331 Vösendorf

D-60528 Frankfurt

D-50226 Frechen

A-1020 Wien

A-1160 Wien

A-1120 Wien

A-4925 Schildorn

A-1140 Wien

CH-9000 St. Gallen

D-63069 Offenbach

A-1220 Wien

A-2111 Harmannsdorf

A-1020 Wien

D-88289 Waldburg

A-1120 Wien

D-85368 Wang

D-76646 Bruchsal

A-1070 Wien

A-1020 Wien

D-90411 Nürnberg

D-64380 Rossdorf

D-96052 Bamberg

A-2822 Walpersbach

A-1140 Wien

A-2355 Wr. Neudorf

D-45147 Essen 


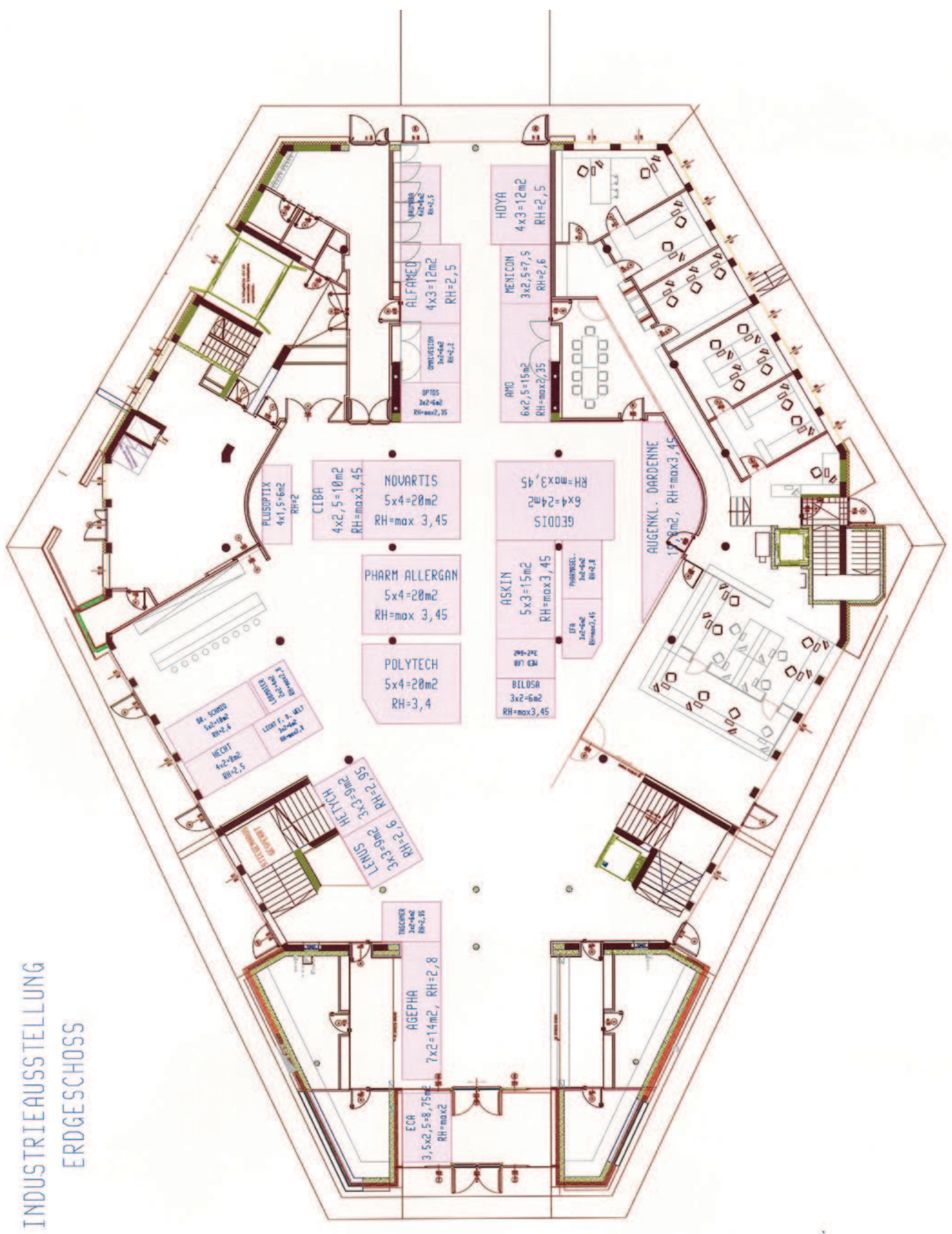




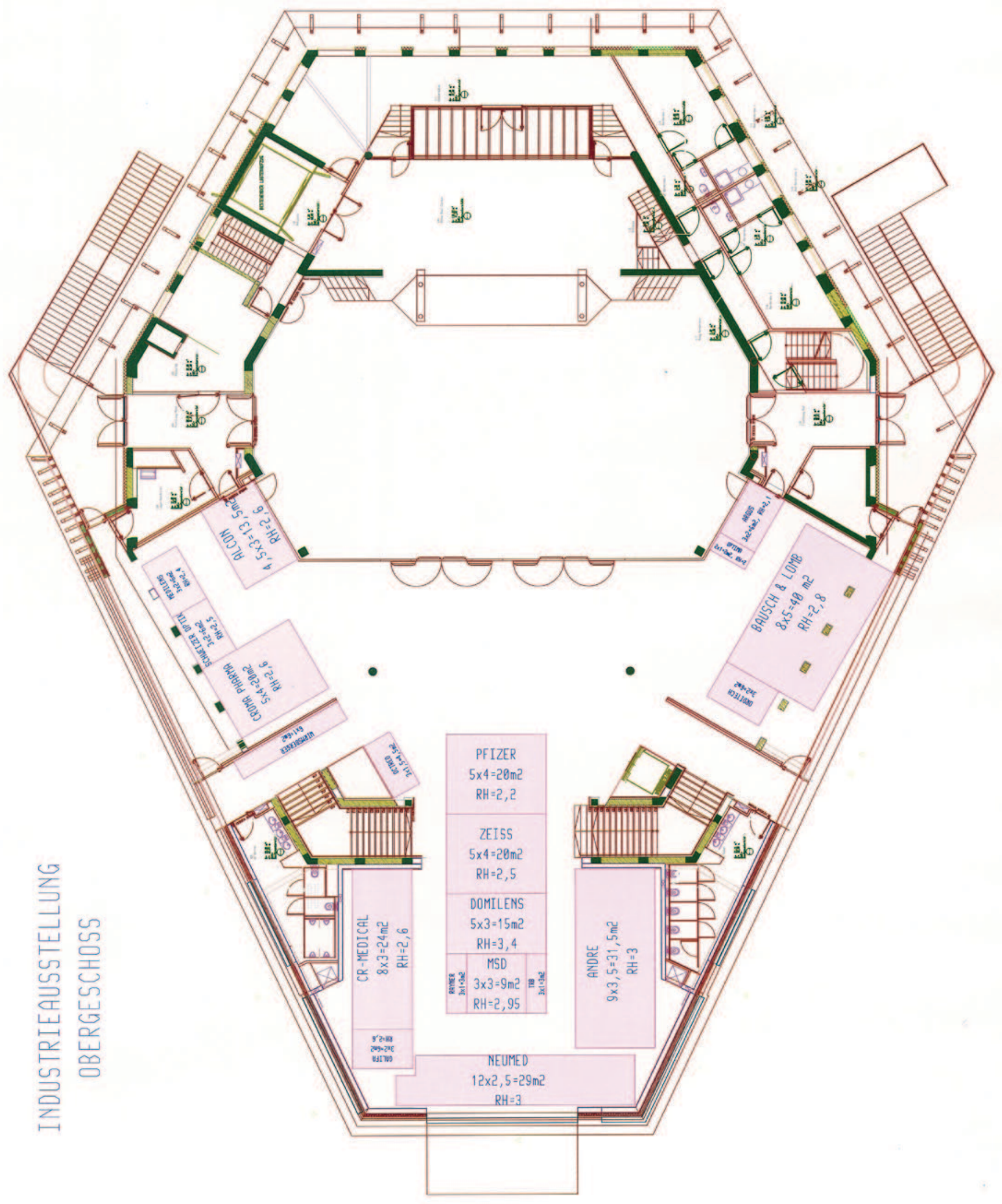




\title{
FORTBILDUNGSMODULE DER ÖOG
}

Für junge Kollegen ist die Facharztprüfung in der EU seit kurzem verpflichtend. Aus diesem Grund hat sich die Österreichische Ophthalmologische Gesellschaft in der Vorstandssitzung 2003 entschlossen eine Reihe von Fortbildungsmodulen anzubieten. Ziel dieser Module ist es, sich ein Spezialwissen anzueignen, das die positive Ablegung der Facharztprüfung erleichtern soll.

\section{Fortbildungsmodul}

\author{
MODUL: Cataract \\ Zeit: $\quad$ Mittwoch, 1. Juni 2011, 16:00 Uhr bis 18:30 Uhr \\ Ort: $\quad$ Europahaus, Saal Kellertheater \\ Organisiert von Prim. Univ.-Prof.Dr. Michael Amon \\ (Katarakt-Kommission der ÖOG) \\ - Anatomie, Linsenphysiologie, Optik (Dr. Alzner) \\ - Embryologie, Cataractogenese (Prof. Faschinger) \\ - Kataraktformen und OP-Indikation (Prof. Schönherr) \\ - $\quad$ Biometrie (Prof. Findl) \\ Pause 15 min \\ - $\quad$ Operationstechnik gestern, heute, morgen (Prof. Amon) \\ - Standard-IOLs und Speziallinsen (Dr. Dexl) \\ - Komplikationen nach Kataraktoperationen (Dr. Neumaier-Ammerer) \\ - OP-Aufklärung (Prof. Radner) \\ - Kataraktoperation und Makula (Dr. Schmidinger) \\ - $\quad$ Spezielle Kataraktformen (Prof. ElShabrawi) \\ Pause $15 \mathrm{~min}$

\section{MODUL: Medizinrecht/ Gutachten} \\ Zeit: $\quad$ Mittwoch, 1. Juni 2011, 18:45 Uhr bis 19:45 Uhr \\ Ort: $\quad$ Europahaus, Saal Kellertheater \\ - Medizinrecht (Prof. Radner) \\ - Führerschein/Fliegerärztliche Untersuchungen (Prof. Vidic) \\ - Gutachterliche Tätigkeiten (Prof. Schauersberger)
}

\section{ÖAC/ÖOG Symposium}

Wirtschaftliche und praktische Aspekte der Augenheilkunde

Zeit: $\quad$ 4. Juni 2011, 13 Uhr 30

Ort: $\quad$ Europahaus, Saal Kellertheater

Kosten: $\quad € 30$,- / für ÖAC Mitglieder kostenlos

Referenten: O. Findl, U. Schönherr, St. Deflorian, P. Ausweger, G. Kieselbach, H. Azem, G. Mader, W. Ecker, A. Vavrovsky, C. Föger, H. Moser

\begin{tabular}{ll} 
Referent & Thema \\
Mag. Stefan Deflorian & $\begin{array}{l}\text { Leistungsorientierte KH Finanzierung: Probleme und Herausforderungen bei der } \\
\text { Abrechnung von Leistungen der Augenheilkunde }\end{array}$ \\
\hline Dr. Wolfgang Ecker & Arzneimittel, Medizinprodukte, HTA/EBM: Aktuelle Aspekte für die Augenheilkunde \\
\hline Dr. Anna Vavrovsky & Gesundheitsökonomische Aspekte in der Augenheilkunde \\
\hline Dr. Ulrich Schönherr & Qualitätssicherung ohne Mehraufwand \\
\hline Dr. Heinz J. Moser & Zivilrechtliche Aspekte der Arzthaftung \\
\hline & Pause \\
\hline Dr. Oliver Findl & Facebook \& Co: Was bringt's für die Ordination? \\
\hline Mag. Christian Föger & Werbebestimmungen für Ärzte \\
\hline Dr. Gabriele Mader & Steuerliche und wirtschaftliche Aspekte in der Augenarztpraxis \\
\hline Dr. Gerhard Kieselbach & OP Termine Österreich und Deutschland, Ergebnisse von "mystery calls" \\
\hline Mag. Barbara Grasser & Integration niedergelassener Augenärzte in eine Augenabteilung \\
\hline Dr. Helga Azem & Über die Zukunft der Augenärzte \\
\hline
\end{tabular}




\title{
RAHMENPROGRAMM
}

\author{
Donnerstag, 2. Juni 2011 \\ 14:00 Uhr Stadtrundgang, Erlebnissennerei \\ 18:30 Uhr Gesellschaftsabend mt der Industrie bei Speis', Trank und Musik
}

Freitag, 3. Juni 2011
6:30 Uhr
2. ÖOG-Lauf
$10.00 \mathrm{Uhr}$
Ausflug Schloss Tratzberg, Silberbergwerk

\section{Schloss Tratzberg - Renaissancejuwel unter den Schlössern Österreichs}

Die Burg wurde erstmals im 13. Jahrhundert urkundlich erwähnt und diente als ehemalige Grenzfeste gegen Bayern und schon Kaiser Maximilian I. als Jagdschloss. Jedoch wurde die ursprüngliche Wehrburg im späten 15 Jahrhundert in Folge eines Brandes, völlig zerstört.

Der Kaiser baute Tratzberg nicht wieder auf, sondern tauschte die Ruine gegen ein Schloss der reichen Silberbergwerksbesitzer Tänzel. Diese errichteten im Jahre 1500 den ersten spätgotischen Teil des heutigen Schlosses Tratzberg in ungewöhnlich prunkvoller, verschwenderischer Weise und ließen es mit außerordentlich kunstvoll gestalteten Marmor-, Holz- und Eisenarbeiten ausstatten.

1554 erwarb der wohlhabende Augsburger Kaufmann Georg Ritter von Ilsung das Schloss, erweiterte und veränderte Tratzberg geprägt durch den Zeitgeist der Renaissance. Zeugnis dafür ist der prachtvoll bemalte Innenhof und erlesene Renaissancezimmer.

Im Erbgang wurde Tratzberg 1590 Besitz des bekannten und reichen Kaufmannsgeschlechts der Fugger, welche das Schloss weiter ausstatteten. Aus dieser Zeit stammt größtenteils das bis heute noch erhaltene Inventar.

Es folgte nach mehreren Besitzerwechseln eine Zeit, in der Tratzberg fast 150 Jahre unbewohnt blieb. Durch Heirat des Franz Graf Enzenberg mit Ottilie Gräfin Tannenberg, ging das mittlerweile fast verwahrloste Schloss im Jahre 1847, in den Besitz der Grafen Enzenberg über, deren privater Wohnsitz es bis heute blieb.

Bis zum heutigen Tage ist es nur dem großen Einsatz der Familie zu verdanken, dass das ca. 6800 qm große Tratzberg, mit seinem 5000 qm Schindeldach, wieder zum Inbegriff eines Tiroler Schlosses des 16. Jahrhunderts und damit zu einem der bedeutendsten Kunst- und Kulturdenkmälern des Landes wurde und der breiten Öffentlichkeit zur Besichtigung zugänglich ist.

\section{Schwazer Silberbergwerk - Das Ausflugsziel in Tirol}

Die „Mutter aller Bergwerke“ verspricht Ihnen eine spannende Reise in die Geschichte Europas. Das größte Silberbergwerk des Mittelalters ist „Die“ Sehenswürdigkeit in Tirol.

Nach der 800 Meter tiefen Einfahrt mit der Grubenbahn erwartet Sie ein außergewöhnliches Erlebnis unter Tage. Folgen Sie den Spuren der Bergleute, die vor über 500 Jahren Silber und Kupfer abbauten und lassen Sie sich in eine Zeit entführen, als die Erde noch eine Scheibe war ...

Sie erfahren interessante Details zur gesellschaftlichen und finanziellen Entwicklung Europas. Sie erhalten darüber hinaus verblüffende statistische Informationen zur Lebensweise von damals.

Besonders bei großer Hitze im Sommer erfahren Sie eine herrliche Abkühlung im Silberbergwerk. Im inneren des Silberbergwerks herrschen konstant 12 Grad Celsius. 

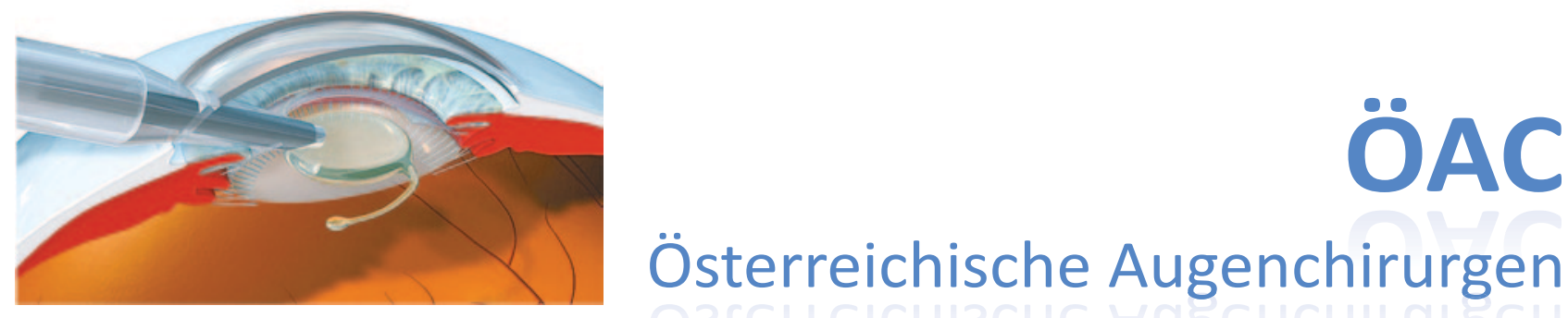

\section{Der Verein Österreichischer Augenchirurgen vertritt die Interessen der operierenden Augenärzte gegenüber verschiedenen Gremien.}

\section{Bis jetzt ist es uns gelungen als geschlossene Gruppe effizient $\mathrm{zu}$ verhandeln.}

\section{Nur mit Ihrem Beitritt kann es uns gelingen, als geeinte Kraft zukünftige Rückschläge zu vermeiden, und uns leistungsentsprechend zu positionieren.}

Obmann: G. Kieselbach • Obmann Stv.: S. Binder

Sekretär: N. Bechrakis • Sekretär Stv.: P. Vécsei-Marlovits

Kassier: A. Wedrich • Kassier Stv.: U. Klemen, U. Schönherr, K. Miller

Kooptierter Vorstand: M. Amon, E. Alzner, Y. El Shabrawi, A. Ettl, R. Fellner, O. Findl, G. Grabner,

M. Radda, U. Schmidt-Erfurth

Vereinsadresse: Gerhard Kieselbach, Anichstraße 35, 6020 Innsbruck

E-Mail: Gerhard.Kieselbach@uibk.ac.at• Tel.: 0512/504 41 84, Fax: 0512/504 3740

ÖAC-Vollversammlung

Termin: Samstag, 4. Juni 2011 um 17 Uhr

Ort: Europahaus, Kellertheater

\section{VORANKÜNDIGUNG \\ 53. ÖOG-Jahrestagung 2012}

17. bis 19. Mai 2012

Villach/Kärnten 